\title{
Fuzzy-Logic Based QoS and Fault Diagnosis Mechanisms for Broadband Satellite Networks under Dynamic Weather Conditions
}

By

\author{
Jiesheng Zhu \\ A thesis submitted to \\ The Faculty of Graduate Studies and Research \\ in partial fulfilment of \\ the degree requirements of \\ Master of Applied Science in Electrical Engineering \\ Ottawa-Carleton Institute for \\ Electrical and System Engineering \\ Department of Systems and Computer Engineering \\ Carleton University \\ Ottawa, Ontario, Canada \\ May 2008 \\ Copyright (C) \\ 2008 - Jiesheng Zhu
}




$\begin{array}{ll}\begin{array}{l}\text { Library and } \\ \text { Archives Canada }\end{array} & \begin{array}{l}\text { Bibliothèque et } \\ \text { Archives Canada }\end{array} \\ \begin{array}{l}\text { Published Heritage } \\ \text { Branch }\end{array} & \begin{array}{l}\text { Direction du } \\ \text { Patrimoine de l'édition }\end{array} \\ \begin{array}{l}\text { 395 Wellington Street } \\ \text { Ottawa ON K1A 0N4 } \\ \text { Canada }\end{array} & \begin{array}{l}\text { 395, rue Wellington } \\ \text { Ottawa ON K1A 0N4 } \\ \text { Canada }\end{array}\end{array}$

Your file Votre référence ISBN: 978-0-494-44067-4 Ourfile Notre référence ISBN: 978-0-494-44067-4

NOTICE:

The author has granted a nonexclusive license allowing Library and Archives Canada to reproduce, publish, archive, preserve, conserve, communicate to the public by telecommunication or on the Internet, loan, distribute and sell theses worldwide, for commercial or noncommercial purposes, in microform, paper, electronic and/or any other formats.

The author retains copyright ownership and moral rights in this thesis. Neither the thesis nor substantial extracts from it may be printed or otherwise reproduced without the author's permission.
AVIS:

L'auteur a accordé une licence non exclusive permettant à la Bibliothèque et Archives Canada de reproduire, publier, archiver, sauvegarder, conserver, transmettre au public par télécommunication ou par l'Internet, prêter, distribuer et vendre des thèses partout dans le monde, à des fins commerciales ou autres, sur support microforme, papier, électronique et/ou autres formats.

L'auteur conserve la propriété du droit d'auteur et des droits moraux qui protège cette thèse. $\mathrm{Ni}$ la thèse ni des extraits substantiels de celle-ci ne doivent être imprimés ou autrement reproduits sans son autorisation.
In compliance with the Canadian Privacy Act some supporting forms may have been removed from this thesis.

While these forms may be included in the document page count, their removal does not represent any loss of content from the thesis.
Conformément à la loi canadienne sur la protection de la vie privée, quelques formulaires secondaires ont été enlevés de cette thèse.

Bien que ces formulaires aient inclus dans la pagination, il n'y aura aucun contenu manquant.

\section{Canada}




\section{Abstract}

In this thesis, two techniques were developed to overcome the problems associated with broadband satellite communication systems. The congestion adaptation system was designed to improve the quality of service for satellite channels under dynamic weather conditions. Different from traditional congestion control techniques, this system uses the rain/snow-fall rate to tune system parameters. Instead of using an algorithm-based tuning method, this system utilizes a knowledge-based method to achieve congestion control in the territorial IP network. The knowledge base was built upon the knowledge of experienced network engineers. Test results indicated robust transmission of high priority traffic under dynamic rain/snow-fall rates.

The knowledge-based terminal fault diagnosis system was designed to diagnose defective terminals. The fault sources of satellite ground terminals were analysed, and the operation conditions of ground terminals were classified into five categories. Both statistical approach and knowledge-based approach were used to diagnose ground terminals. The overall diagnosis accuracy is satisfactory, and the effectiveness of the system was approved by a satellite service provider. 
To my parents 


\section{Acknowledgements}

I would like to thank my thesis supervisor, Professor C. Huang for his guidance and encouragement throughout the period of my study. I gratefully acknowledge Mr. Kalai Kalaichelvan of EION, Mr. Bharat Rudra of OCE, and Mr. Derek Best from Precarn for supporting this project. In particularly, I wish to thank Mr. Andre Bigras, Mr. Abdul Lakhani, and Mr. Michael Roseberg from Telesat for providing valuable data and verifying the applicability and usefulness of this application. 


\section{Table of Contents}

$\begin{array}{ll}\text { Abstract } & \text { iii }\end{array}$

Acknowledgements $\quad$ v

Table of Contents vi

List of Tables $\quad$ ix

List of Figures $\quad x$

List of Acronyms $\quad$ xiii

Chapter 1 Introduction 1

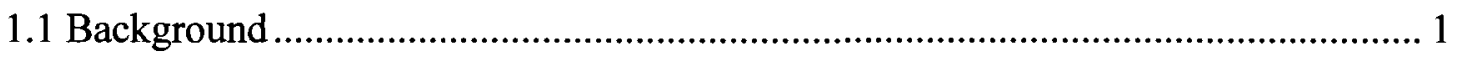

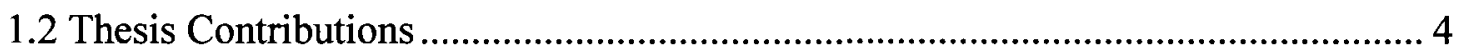

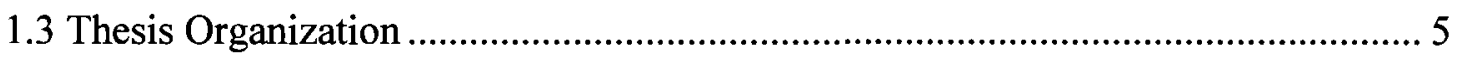

Chapter 2 Review of the State of the Art $\quad 7$

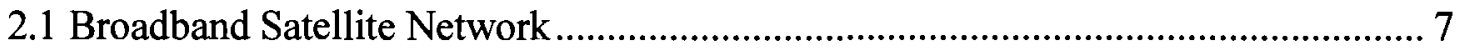

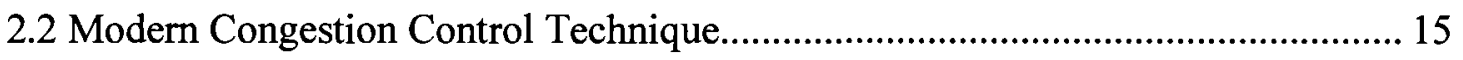

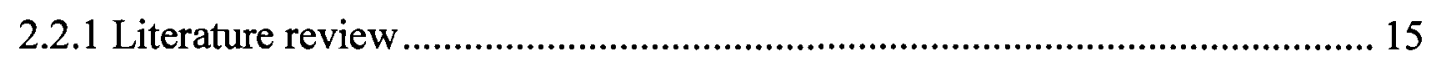

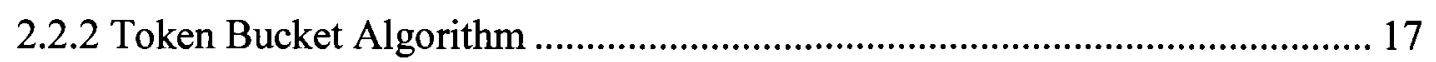

2.2.3 Random Early Detect (RED) Algorithm.................................................. 19

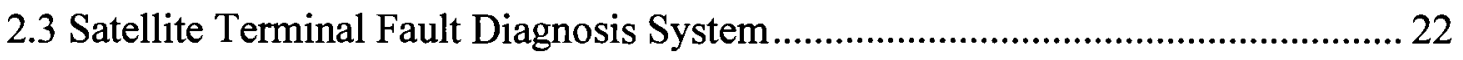

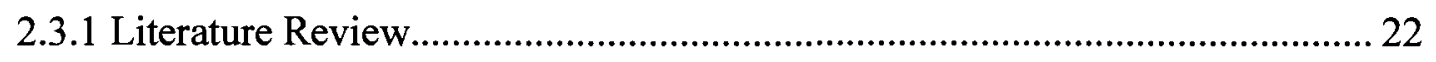

$\begin{array}{ll}\text { Chapter } 3 \text { Congestion Adaptation System } & 24\end{array}$ 


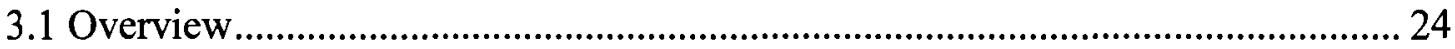

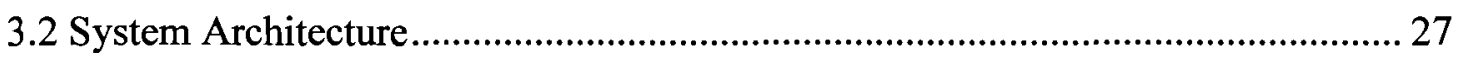

3.3 Packet Conditioning......................................................................................... 30

3.4 Functionality of Traffic Shaper............................................................................ 32

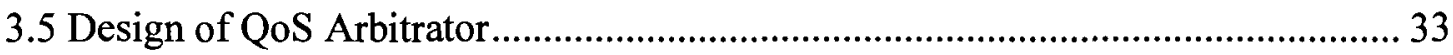

3.5.1 Mapping Input/Output Variable into Fuzzy Sets ............................................ 33

3.5.2 Constructing Knowledge Base.......................................................................... 37

Chapter 4 Knowledge-based Terminal Fault Diagnosis System 42

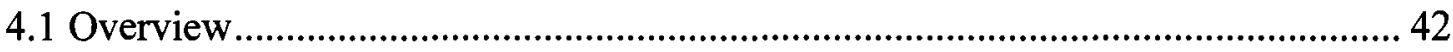

4.2 System Architecture .......................................................................................... 45

4.3 Mapping Forward Link SNR pattern to Events ........................................................ 47

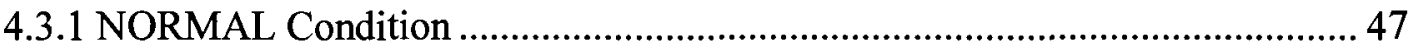

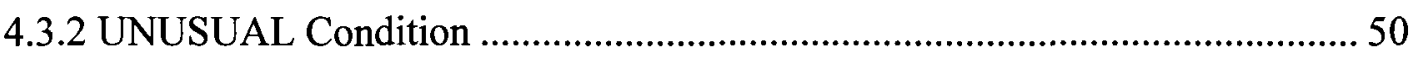

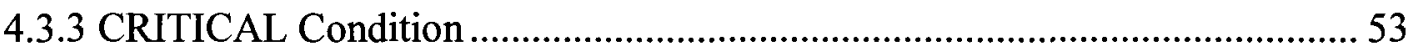

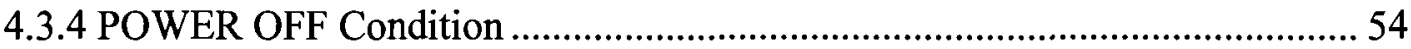

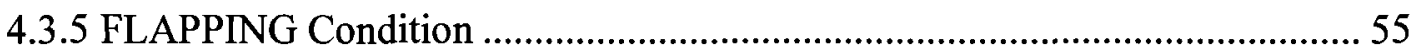

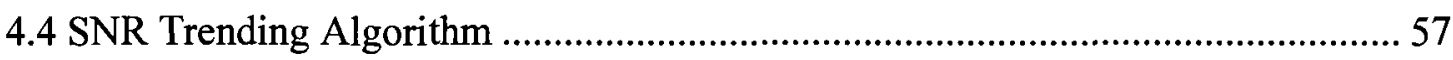

4.4.1 SNR Trending Algorithm ………………………..................................... 57

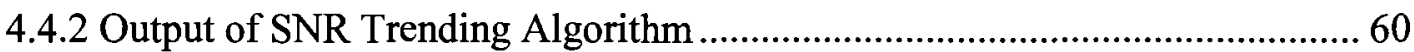

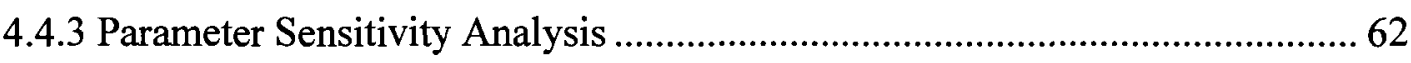

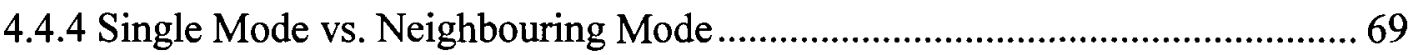

4.5 Maximum Likelihood Decision Making............................................................... 73

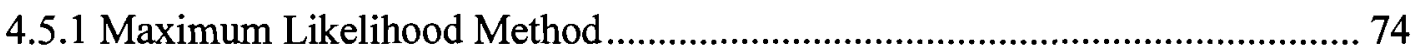

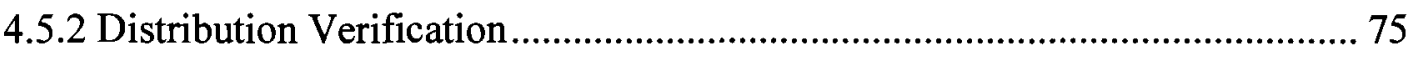

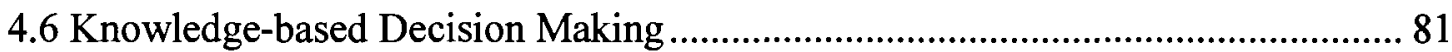

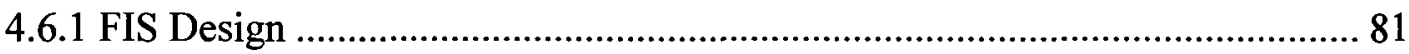

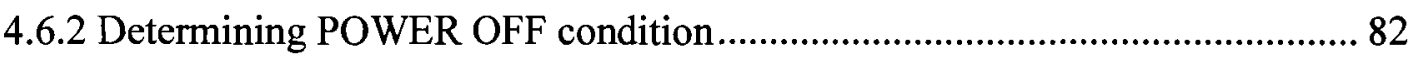

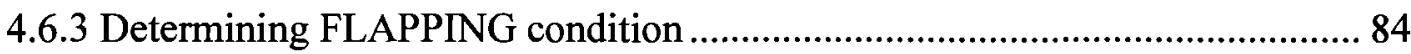

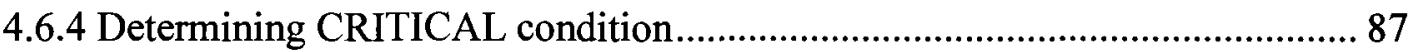

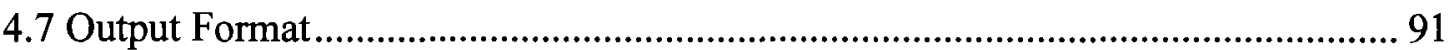




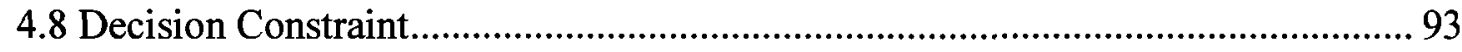

$\begin{array}{ll}\text { Chapter } 5 \text { Test Results and Discussions } & 97\end{array}$

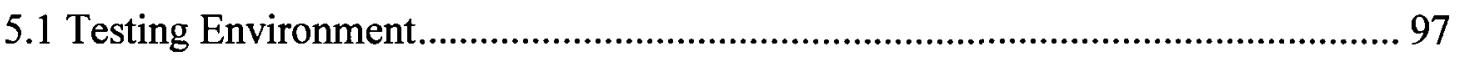

5.2 Testing Results for Congestion Adaptation System ................................................. 99

5.2.1 Testing of Traffic Shaper ................................................................................... 99

5.2.2 Testing of enhanced QoS policy ..................................................................... 100

5.3 Testing Results for Knowledge-based TFD System ............................................... 102

5.3.1 Diagnosis of POWER OFF Condition.......................................................... 102

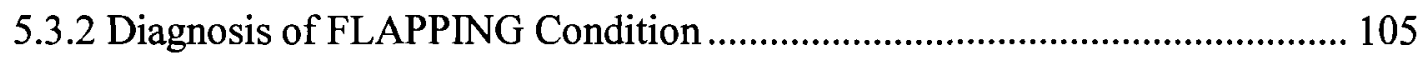

5.3.3 Performance of Knowledge-based TFD System under Difference Decision

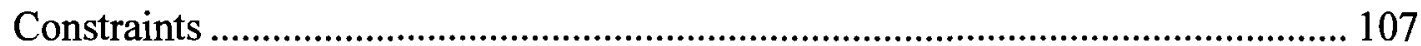

5.3.4 Diagnosis Accuracy ................................................................................ 112

5.3.5 Performance Comparison of Single Mode and Neighbouring Mode ............. 116

Chapter 6 Conclusions and Suggestions for Future Work 120

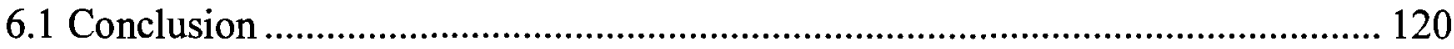

6.2 Suggestions for Future Work …………………………..................................... 123

Appendix A: Anik-F2 Specification and Coverage Map 124

Appendix B: Satellite Terminal Locations $\quad 126$

$\begin{array}{ll}\text { Appendix C: Fuzzy Logic Theory } & 127\end{array}$

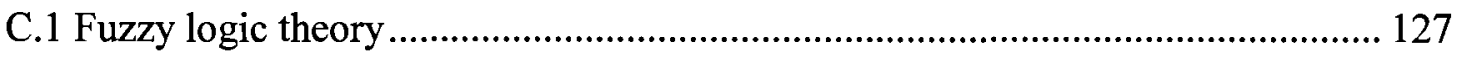

C.2 Fuzzy Inference System ................................................................................ 135

Appendix D: Historical Forward Link SNR 142

References 


\section{List of Tables}

Table 2.1: RF trending database parameters................................................................. 13

Table 2.2: Sample RF performance data log file ........................................................ 14

Table 3.1: Possible values of precedence bits and their meanings .................................... 31

Table 3.2: Possible values of other ToS bits and their meanings ....................................... 31

Table 3.3: Rule set for QoS Arbitrator............................................................................ 38

Table 4.1: Probability distributions of NORMAL/UNUSUAL condition under single

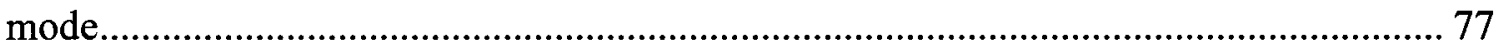

Table 4.2: Probability distributions of NORMAL/UNUSUAL condition under

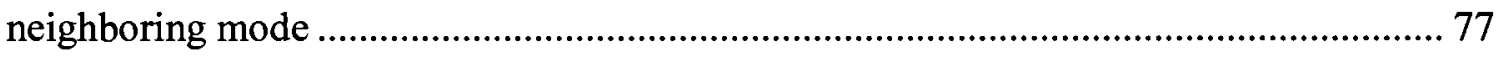

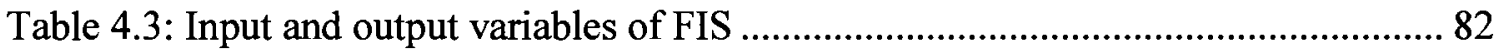

Table 4.4: Rule set for diagnosing POWER OFF condition.............................................. 84

Table 4.5: Rule set for diagnosing FLAPPING condition................................................. 86

Table 4.6: Rule set for diagnosing CRITICAL condition.............................................. 89

Table 4.7: Knowledge-based TFD system output parameters ............................................. 92

Table 4.8: Output format of knowledge-based TFD system............................................. 92

Table 4.9: Sample outputs of knowledge-based TFD system.............................................. 94

Table 4.10: Diagnosis symptoms for sample outputs under different decision constraints

Table 5.1: Transmission rate of priority traffic and non-priority traffic under different

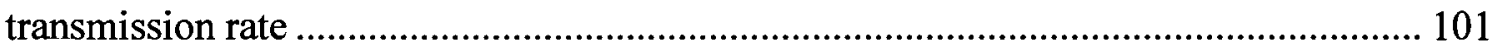

Table 5.2: Diagnosis result of Altin station under difference decision constraints ........ 111

Table 5.3: Diagnosis success ratios of knowledge-based TFD system ............................ 113

Table 5.4: Diagnosis false alarm ratios of knowledge-based TFD system...................... 114 


\section{List of Figures}

Figure 2.1: Network architecture of a broadband satellite network.................................... 8

Figure 2.2: Illustrating communication paths for satellite channel...................................... 9

Figure 2.3: Forward link SNR for Terrace station during a day ......................................... 12

Figure 2.4: Functional diagram for token bucket algorithm [27] ................................... 18

Figure 2.5: Flowchart for RED algorithm [19] .......................................................... 20

Figure 3.1: Congestion adaptation system software stack ................................................. 28

Figure 3.2: System architecture of congestion adaptation system .................................... 29

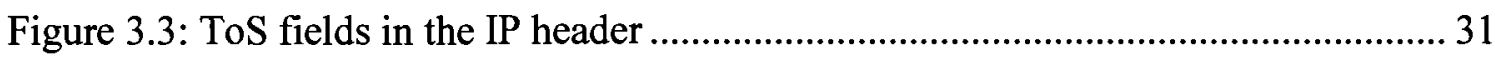

Figure 3.4: Membership functions for rain/snow-fall rate using traditional mapping

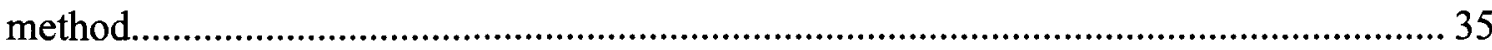

Figure 3.5: Membership functions for rain/snow-fall rate.............................................. 35

Figure 3.6: Membership functions for maximum transmission rate.................................. 36

Figure 3.7: Membership functions for maximum queue threshold..................................... 36

Figure 3.8: Membership functions for maximum dropping probability ............................ 37

Figure 3.9: Output range of maximum transmission rate .................................................. 38

Figure 3.10: Output range of maximum queue threshold ................................................. 39

Figure 3.11: Output range of maximum dropping probability ............................................ 39

Figure 3.12: Determining QoS parameters using fuzzy logic controller ........................... 40

Figure 4.1: System architecture of knowledge-based TFD system ................................... 46

Figure 4.2: FLS with daily variation pattern under NORMAL condition .......................... 49

Figure 4.3: FLS with rain fade pattern under NORMAL condition .................................. 49

Figure 4.4: Deteriorating performance of Arviat station under UNUSUAL condition.... 52

Figure 4.5: Deteriorating performance of Altin station caused by antenna displacement 52

Figure 4.6 FLS performance under satellite manouvre ...................................................... 52 
Figure 4.7: Deteriorating performance of Pond Inlet station under CRITICAL condition 54

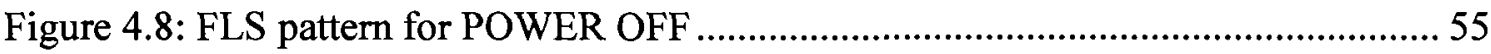

Figure 4.9: Temporary failure of pulling $\mathrm{RF}$ data due to extreme weather condition...... 55

Figure 4.10: FLS pattern for flapping modem ............................................................... 56

Figure 4.11: Relationship between Sampling Period and Sliding Window ..................... 58

Figure 4.12: FLS of Hazelton station ( 3 hour average) ....................................................6 63

Figure 4.13: Measurement of Terminal State under different $\mathrm{SW}$ with $\mathrm{SP}=3$ hours...... 66

Figure 4.14: Measurement of Terminal State under different SP with SW $=14$ days ..... 67

Figure 4.15: Measurement of Terminal State under different SP and SW ......................68

Figure 4.16: Terminal State of Hazelton and Terrace station using single mode............. 72

Figure 4.17: TS Difference of Hazelton station using neighboring mode........................ 72

Figure 4.18: Illustrating the hypothesis and decision boundary of NORMAL/UNUSUAL condition 75

Figure 4.19: Q-Q plot comparing the distribution of TS under NORMAL condition and exponential distribution (single mode)

Figure 4.20: Q-Q plot comparing the distribution of TS under UNUSUAL condition and normal distribution (single mode) 78

Figure 4.21: Probability distribution of NORMAL/UNUSUAL condition for single mode 78

Figure 4.22: Q-Q plot comparing distribution of TS difference under NORMAL condition and normal distribution (neighboring mode) 79

Figure 4.23: Q-Q plot comparing distribution of TS difference under UNUSUAL condition and normal distribution (neighboring mode) 79

Figure 4.24: Probability distribution of NORMAL/UNUSUAL condition for neighboring mode. 80

Figure 4.25: Membership functions for $\mathrm{ZC}$............................................................... 83

Figure 4.26: Membership functions for POWER OFF Indicator ...................................... 83

Figure 4.27: Decision curve of diagnosing POWER OFF condition.................................. 84

Figure 4.28: Membership functions for FI.................................................................. 85 
Figure 4.29: Membership functions for FLAPPING Indicator.

Figure 4.30: Decision surface of diagnosing FLAPPING condition ( $\mathrm{x}$-axis=FI, $\mathrm{y}-$ axis $=\mathrm{ZC}$ ) .86

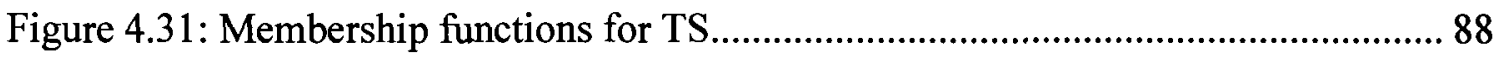

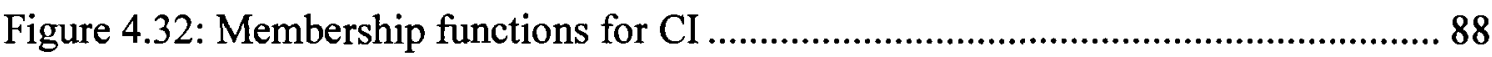

Figure 4.33: Membership functions for CRITICAL Indicator .......................................... 89

Figure 4.34: Decision surface of diagnosing CRITICAL condition part 1

$(\mathrm{x}$-axis $=\mathrm{CI}$, y-axis $=\mathrm{ZC})$

Figure 4.35: Decision surface of diagnosing CRITICAL condition part 2

( $\mathrm{x}$-axis $=\mathrm{ZC}$, $\mathrm{y}$-axis $=\mathrm{TS}$ ) 90

Figure 5.1: Topology of broadband satellite test-bed network .......................................... 98

Figure 5.2: Transmission rate of return link under difference rain/snow-fall rate.......... 100

Figure 5.3: FLS of Hazelton station from May $16^{\text {th }} 2007$ to May $19^{\text {th }} 2007$.................. 103

Figure 5.4: Illustrating the output of FIS under POWER OFF condition........................ 103

Figure 5.5: FLS of Hazelton station from October $22^{\text {nd }}$ to October $25^{\text {th }} 2007$.............. 104

Figure 5.6: Illustrating change of POWER OFF, FLAPPING and CRITICAL indicator 104

Figure 5.7: FLS of Altin station from June $16^{\text {th }} 2007$ to June $19^{\text {th }} 2007 \ldots \ldots \ldots \ldots \ldots \ldots . . . . . . . . .106$

Figure 5.8: Illustrating change of POWER OFF, FLAPPING, INDICATOR indicators106 Figure 5.9: FLS of Altin station from November 2006 to July 2007 (3 hour average) .. 110 Figure 5.10: Illustrating diagnosis result of Altin station under tight constraint 110

Figure 5.11: Illustrating diagnosis result of Altin station using pie chart (a) Nominal Constraint (b) Loose Constraint (c) Tight Constraint

Figure 5.12: FLS of Hazelton station from August 2007 to December 2007 (3 hour average)

Figure 5.13: FLS of Terrace station from August 2007 to December2007 (3 hour average)

Figure 5.14: Comparing diagnosis result of Hazelton station under tight constraint

(a) single mode (b) neighboring mode

Figure 5.15: Illustrating diagnosis result of Hazelton station with tight constraint 119 xii 


\section{List of Acronyms}

$\begin{array}{ll}\text { ACM } & \text { Adaptive Coding and Modulation } \\ \text { CI } & \text { Critical Indicator } \\ \text { CPE } & \text { Customer Premise Equipment } \\ \text { ECN } & \text { Explicit Notification to Congestion } \\ \text { FI } & \text { Flapping Indicator } \\ \text { FIS } & \text { Fuzzy Inference System } \\ \text { FLS } & \text { Forward Link Signal-to-Noise Ratio } \\ \text { GT } & \text { Ground Terminal } \\ \text { Kbps } & \text { Kilo-bits per second } \\ \text { LAN } & \text { Local Area Network } \\ \text { LoS } & \text { Line of Sight } \\ \text { Mbps } & \text { Mega-bits per second } \\ \text { QoS } & \text { Quality of Service } \\ \text { RED } & \text { Random Early Discard } \\ \text { RF } & \text { Radio Frequency } \\ \text { RLS } & \text { Return Link Signal-to-Noise Ratio } \\ \text { SNR } & \text { Signal to Noise Ratio } \\ \text { SP } & \text { Sampling Period } \\ \text { SW } & \text { Sliding Window } \\ \text { TS } & \text { Terminal State } \\ \text { TFD } & \text { Terminal Fault Diagnosis } \\ \text { TCP } & \text { Transport Control Protocol } \\ \text { ToS } & \text { Type of Service } \\ \text { VoIP } & \text { Voice over IP } \\ \text { ZC } & \text { Zero Count } \\ & \end{array}$




\section{Chapter 1}

\section{Introduction}

\subsection{Background}

Due to extensive geographic reach, satellite communication systems are the most promising technology for providing broadband Internet service anytime and anywhere in remote areas where the cost of deploying infrastructure-based network is expensive. A new concept of satellite communication called broadband satellite network was proposed in [1] and [2] to support broadband communications over satellite systems. Broadband satellite networks are typically IP-based and provide a ubiquitous means of communication for multimedia and high data-rate Internet services. In terms of satellite systems, the two types of systems used are overlay satellites and access satellites; although, most proposed broadband satellite systems use overlay satellites [2], which can provide direct user-to-user connectivity via a single hop and transport territorial network traffic. Overlay satellites are equipped with onboard processing units and play a competitive role in the broadband satellite network. However, the first generation of broadband satellites are access satellites [3]. Access satellites operate as channel relay and provide last-mile service to satellite network subscribers.

Broadband satellite systems offer multimedia communication to a large number of users in rural areas using low-cost equipments. In July 2004, Telesat launched Anik-F2, 
which is a broadband satellite manufactured by Boeing. Anik-F2 provided North America's first commercial satellite-based broadband communication service which brings broadband Internet, distance learning, and telemedicine to rural areas in the United States and Canada [4] [5]. Although the broadband satellite network has the remarkable advantage of providing broadband service to remote areas, the satellite service provider encountered some problems in the daily operation of the network. First of all, the channel capacity of the satellite link decreased when remote sites experienced rain/snow-fall. Second, the reliability of satellite networks reduced when the Ground Terminal (GT) experienced Line-of-Sight (LoS) impairments or other faults. In this thesis, two techniques were developed to improve the quality of service and reliability of the broadband satellite network. A congestion adaptation system was developed to regulate IP data traffic and to enhance QoS on priority traffic under inclement weather conditions. The knowledge-based Terminal Fault Diagnosis (TFD) system was developed in order to monitor the operating conditions of GTs, as well as to report back to the satellite service provider with prompt and accurate diagnosis results.

One of the most significant disadvantages of the broadband satellite system is channel degradation due to an increasing rain/snow-fall rate. Broadband satellites use microwaveband signals to communicate with GTs. The microwave signal attenuates faster when travelling through water. A comprehensive study of the relationship between attenuation of microwave signal and rain/snow-fall rate was done in [6]. The research indicated that the higher the rain/snow-fall rate, the more attenuation on the microwave signal. As a result, when the remote GT site experiences rain/snow-fall, the satellite signal will suffer 
extra attenuation before it reaches the GT. The attenuated signal results in channel degradation, thereby leading to network congestion. Moreover, since broadband satellite networks are IP-based networks, the congestion control of the network is crucial for committing Service Level Agreements (SLAs). The objective of the service provider is to develop a system that can prevent the broadband satellite network from congestion while committing SLAs to high priority traffics, such as VoIP packets under dynamic weather conditions.

In this thesis, a congestion adaptation system for a broadband satellite network was proposed in order to enhance QoS under dynamic weather conditions. The congestion adaptation system regulates the maximum data rate entering the satellite network according to the change in weather conditions around the targeted GT. Furthermore, the system enhances the QoS of priority streams by enforcing the priority traffic to go through at the expense of low priority traffic. The congestion adaptation system was developed and deployed in Telesat's broadband satellite test-bed network for verification and testing. The test results confirmed the operability and effectiveness of the proposed solution; however, the congestion adaption system needs to be tuned to meet the requirements for specific broadband satellite systems.

In broadband satellite networks, GTs are deployed in remote sites in order to serve local customers. The service quality and availability of a GT is highly dependent on the receiving Signal-to-Noise Ratio (SNR). The fluctuation and deterioration of SNR could cause GTs to underperform or $\log$ off of the network. Since the transmission power of relay satellites is stable, the fluctuation and deterioration of SNR would most likely be 
caused by LoS impairments and various terminal faults. The plotting of different SNRs illustrated specific patterns for different faulty conditions occurring at the remote GT site. In order to match the SNR patterns to the correct events that actually happened on site, satellite service providers need to have experienced satellite operators analyze the measured SNR at GT. As a result, the fault identification process is highly dependent on human expertise and expert availability, and unfortunately, it is being inefficiently executed. The objective of the fault diagnosis system is to provide accurate diagnosis results on the operating conditions of GTs, and to use it to assist satellite service providers or customers to quickly fix the fault.

This thesis proposes a knowledge-based TFD system that monitors the trends of the SNR and matches the SNR behaviour to pre-defined faulty conditions based on a set of rules, which are translated from knowledge of experienced satellite operators. The knowledge-based TFD system is intelligent, effective, and upgradeable. The diagnostic results were assessed by satellite service providers, and they were accurate enough to be qualified as expert decisions.

\subsection{Thesis Contributions}

The contributions of this thesis are:

1. Proposed a new fuzzy-logic based congestion adaptation system for a broadband satellite network that regulates the traffic and maintains the QoS for high priority traffic dynamically under inclement weather conditions.

a) Proposed to use rain/snow-fall rate to tune the system parameters, in order to achieve congestion control under inclement weather conditions. 
b) Selected proper membership functions to accurately represent weather conditions.

c) Created a unique rule set that is derived from experienced satellite operators and network engineers.

2. Proposed a novel knowledge-based TFD system that is able to successfully diagnosis operating conditions of satellite GTs.

a) Introduced a knowledge-based fault diagnosis system to diagnose the operating conditions of a satellite GT.

b) Developed a sliding window-based SNR trending algorithm to extract SNR trending information.

c) Used a Maximum Likelihood method to make decisions based on statistically estimations.

d) Constructed a fuzzy-logic based knowledge system to reproduce expert knowledge in a systematic manner and created unique rule sets to diagnose operating conditions of satellite GTs.

3. Designed and implemented both systems.

4. Successfully tested both systems in a broadband satellite network test-bed.

\subsection{Thesis Organization}

The remaining chapters of this thesis are organized as followed:

Chapter 2: Introduces the basics of broadband satellite networks and reviews some of the classical theories adopted as solutions for our system. 
Chapter 3: Describes the system architecture of the congestion adaptation system and explains its major functionalities in detail.

Chapter 4: Maps various RF signal patterns into different phenomena and presents the design of a knowledge-based TFD system and discusses the design of knowledge-based TFD system in detail.

Chapter 5: Explains the testing environment and shows the testing results for the both congestion adaptation system and knowledge-based TFD system.

Chapter 6: Presents the conclusion and recommendations for future research. 


\section{Chapter 2}

\section{Review of the State of the Art}

\subsection{Broadband Satellite Network}

The architecture of an IP-based broadband satellite network is illustrated in Figure 2.1. The network follows a star topology and consists of a satellite hub, several GTs, and a broadband satellite. The broadband satellite has a bent-pipe architecture and works as a channel relay. In the network, GTs serve as a gateway between satellite networks and local area networks. GTs have a full-duplex data communication channel with the satellite hub. All GTs communicate with the satellite hub via satellite relay. If two GTs want to communicate with each other, the data has to go through the satellite hub. Channel allocation, transmission power control, Adaptive Coding and Modulation (ACM) functionalities, and SLA settings are performed in the satellite hub, which connects the satellite network to the Internet. The congestion adaptation system will be installed in both the satellite hub and the GTs in the network. The knowledge-based TFD system will also be implemented in the satellite hub. 


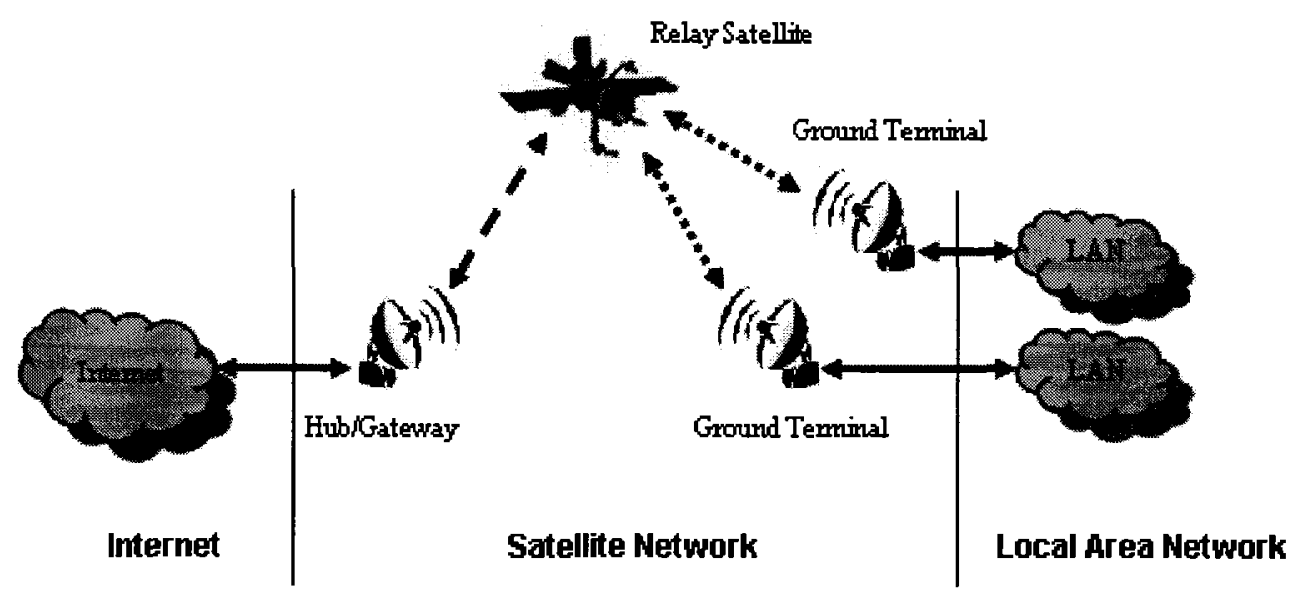

Figure 2.1: Network architecture of a broadband satellite network

Geostationary broadband satellite Anik-F2 is the heart of the broadband satellite network. Telesat's Anik-F2 satellite is a tri-band satellite, with $38-\mathrm{Ka}, 32-\mathrm{Ku}$, and $24-\mathrm{C}$ band transponders. It was one of the world's largest commercial communication satellites when it was launched in July 2004. Anik-F2 is the first of its kind to provide Ka-band multimedia and broadband Internet services across North America. The Ka-band spectrum is a Super High Frequency (SHF) band where a 20/30 GHz band is used for satellite communication. More specifically, Anik-F2 uses a $20 \mathrm{GHz}$ band for downlink (satellite to GT), and a $30 \mathrm{GHz}$ band for uplink (GT to satellite). A pair of uplink and downlink form a satellite channel. The communication path from the satellite hub to the GT is called a forward link. The path from a GT to satellite hub is called return link. The communication paths of satellite channel are illustrated in Figure 2.2. Like the C-band and Ku-band, the Ka-band spectrum offers three advantages over low frequency bands. The advantages can be summarized as spectrum availability, reduced interference, and reduced equipment size [9]. More available spectrums resulted in high throughput of the 
system. In addition, Anik-F2 uses spot beam technology for Ka-band signals, which allows the satellite to provide robust service to specific areas on the Earth's surface. Spot beam technology also allows the satellite to reuse the same frequency in order to transmit signals to different areas. The ability for a satellite to reuse the same frequencies for different geographical areas without interfering with each other at the receiver enabled simplified receiver designs. Due to the reduced equipment size and simplified receiver design, low-cost equipment has become available and more affordable for many families. The specifications of Anik-F2 and the Ka-band spot beam coverage map are shown in Appendix A. However, some inbred disadvantages of Anik-F2 have limited the scope of this project. For example, Anik-F2 has a fixed architecture where the power and bandwidth can not be changed and the bandwidth can not be reallocated either statically or dynamically between spot-beams. This feature disallows the reallocation or sharing of bandwidth between spot beams when some service areas undergo network congestion.

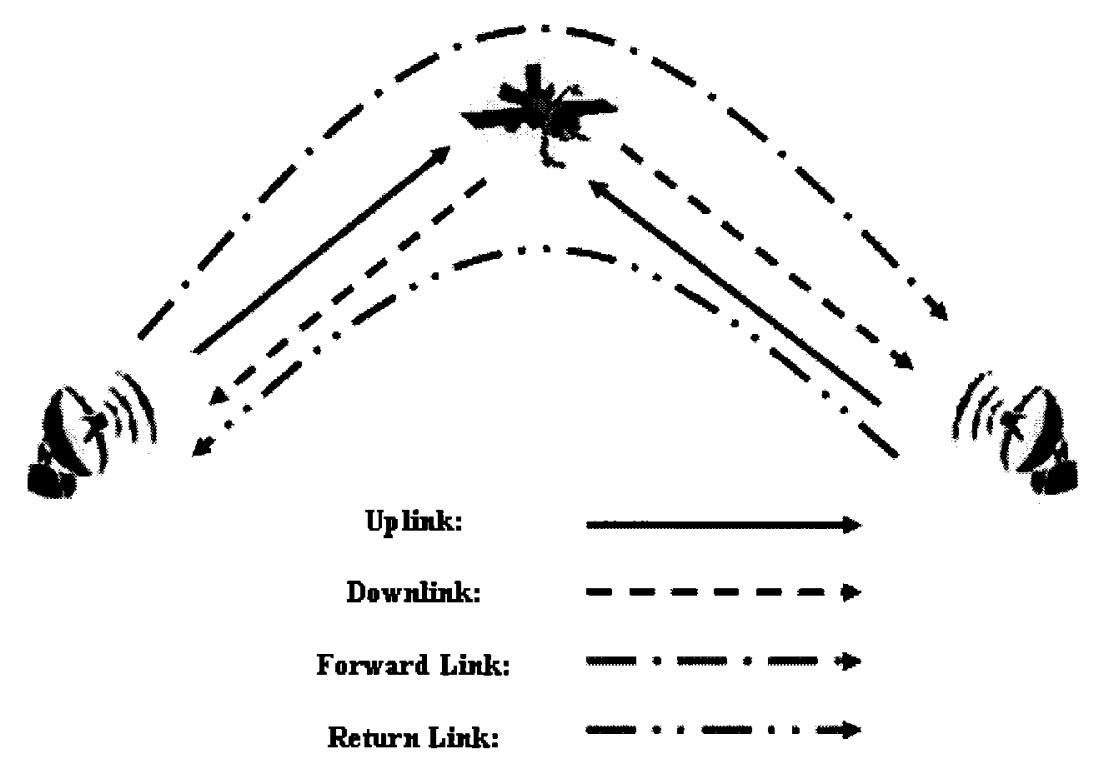

Figure 2.2: Illustrating communication paths for satellite channel 
Using a Ka-band signal to provide broadband satellite service was a technological breakthrough, and it brought remarkable advantages over existing satellite frequency bands; however, the benefits of using a Ka-band came with tradeoffs. The primary problem geostationary satellite faced was channel latency. Geostationary orbit is a synchronous orbit located above the Earth's equator, approximately $35,786 \mathrm{~km}$ above sea level. The altitude of the satellite orbit results in significant propagation delay for the satellite signal. In this thesis, the round trip delay of the broadband satellite network is about $580 \mathrm{~ms}$.

A comprehensive study of propagation issues of the Ka-band spectrum in [9] and [10] showed the propagation effects of a Ka-band signal. The primary sources of LoS impairment on Ka-band signals are summarized below:

Rain Attenuation: Signal attenuation due to rain is the most severe impairment for Ka-band signals. The rain attenuation could exceed $20 \mathrm{~dB}$ in a short period of time.

Gaseous Absorption: Usually associated with oxygen and water vapour absorption. The lost of signal strength is approximately to $1 \mathrm{~dB}$.

Cloud Attenuation: Ka-band signal propagating through clouds loses $1 \mathrm{~dB}$ or more. Scintillation: Mainly caused by the time-varying changes in the refractive index of the atmosphere. The consequence of this is rapid fluctuations of signal amplitude and phase.

Atmospheric Noise: The atmospheric noise has an equivalent black body temperature, which varies from $10 \mathrm{~K}$ to ambient temperature. 
Wet Antenna or Snow on Antenna: Condensation and snow on the antenna cause signal losses. This kind of losses can be as large as a few $\mathrm{dB}$.

Among these propagation effects, rain attenuation is the most dominant impairment for Ka-band signals. The rain attenuation effect can be captured by monitoring the receiving signal SNR of a GT. Figure 2.3 shows the forward link SNR of Terrace station from 15:57 August 30, 2007 until 15:57 August 31, 2007. The forward link SNR varies between $11.2 \mathrm{~dB}$ and $7 \mathrm{~dB}$. When a GT site is experiencing high rain/snow-fall over a short period of time, the forward link SNR will drop fast compared to its expected performance level. As shown in Figure 2.3, the weather impact can be identified, since the forward link SNR dropped rapidly at around 7:00 am in the morning. The forward link SNR dropped from $9 \mathrm{~dB}$ to as low as $7 \mathrm{~dB}$ in a short period of time, then it started to increase slowly, and gradually recovered to $9 \mathrm{~dB}$ at around 10:00 am. Since the satellite system uses an ACM scheme, the link capacity of the broadband satellite system relies heavily on the quality of wireless signal. A small degradation in the signal strength will result in reduced link capacity of the satellite channel. Therefore, inclement weather conditions on site will cause network congestion and lead to failure to meet QoS requirements. The main objective of the congestion adaptation system is to protect the broadband satellite network from congestion under dynamic weather conditions. 


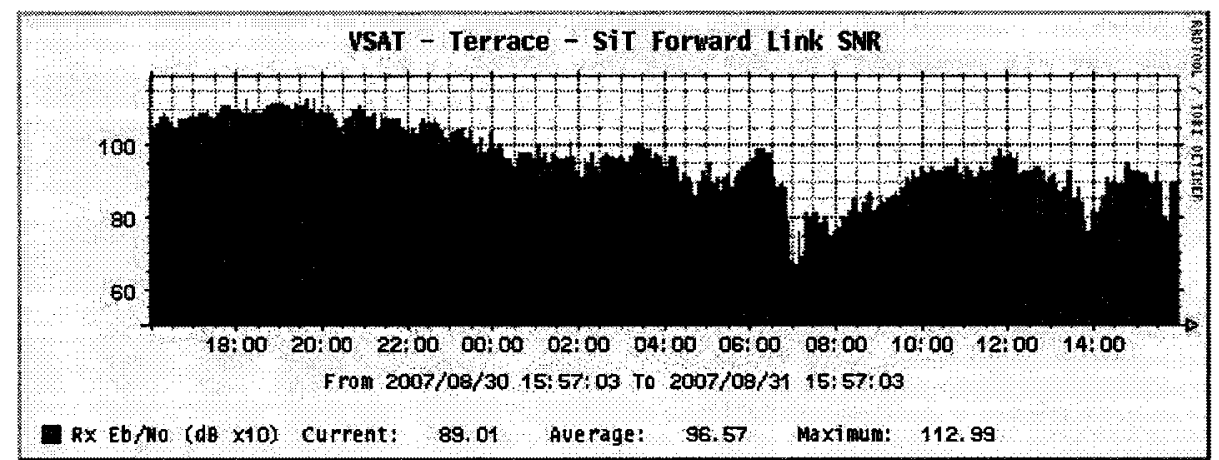

Figure 2.3: Forward link SNR for Terrace station during a day

A satellite signal travelling through the air experiences attenuations from all sources of propagation effects. When the attenuation is dominated by one of the propagation effects, it causes the GTs to underperform or temporarily go out of service. The knowledge-based TFD system is designed to report the operating conditions of the GTs according to analysis of historical forward link SNR.

In order to support the development of the congestion adaptation system and knowledge-based TFD system, a RF performance trending database is maintained at the satellite hub. The RF performance metrics from four remote sites located on the west coast of Canada were monitored. The four GT sites are Altin in British Columbia, Hazelton in British Columbia, Yellowknife in Northwest Territories, and Terrace in British Columbia. The geographical locations, meaning the altitude and longitude of these sites, are shown in Appendix B. In addition to the four GT sites, the locations of six other GT sites are also listed in Appendix B. RF performance related data points collected are displayed in Table 2.1. RF performance metrics are divided into two categories: return link performance data set and forward link performance data set. The return link performance data set describes the operating condition of remote GT. It 
consists of Return Link SNR, Return Link Power, Return Link Frequency, Return Link Modulation, and Return Link Code-Point. The Return Link SNR represents the signal quality of downlink for return link. Return link SNR is calculated using measurements taken from the demodulator chipsets located in the satellite hub. The Return Link Power is the transmission power of the uplink signal for the return link. Return Link Frequency, Modulation, and Code-Point are the measurements of the uplink signal for the return link path. The Forward link RF data set has the same elements as the return link RF data set with the same designation. The Forward Link SNR is the receiving signal quality calculated at GT. Because the transmission power of the satellite is stable, the forward link SNR calculated at GT reflects the channel condition. The source of LoS impairment can be identified by analysing the trends of forward link SNR. As a result, the forward link SNR is an important indicator for the operation condition of a GT. A sample log file format used to store the RF performance parameters collected from Altin station is illustrated in Table 2.2 .

Table 2.1: RF trending database parameters

\begin{tabular}{|c|c|c|}
\hline RF Related Parameters & Log Fle Desigaation & Unit \\
\hline Terminal Site Name & IDU & N/A \\
\hline Retum Link SNR & RLS & dB × 10 \\
\hline Return Link Power & RLP & dB \\
\hline Retum Link Frequency & RLF & GHz \\
\hline Retum Link Modulation & RLM & N/A \\
\hline Return Link Code-Point & RLC & N/A \\
\hline Forward Link SNR & FLS & dB x 10 \\
\hline Forward Link Frequency & FLF & GHz \\
\hline Forward Link Modulation & FLM & N/A \\
\hline Forward Link Code-Point & FLC & N/A \\
\hline Date Time Stamp & DTS & MonthDay/Year Time \\
\hline
\end{tabular}


Table 2.2: Sample RF performance data log file

DU RLS RLP RLF RLM RLC FLS FLF FLM FLC DTS

ALN $110 \quad-25 \quad 29.868$ QPSK TC rate $2 / 37928396$ QPS K RS rate 12 06/010700:00

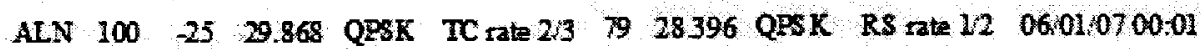

ALN 95 25 29.868 QPSK TC rate $23 \quad 8028.396$ QRSK RS rate $1 / 2 \quad 0601 / 0700.02$

ALN $95 \quad-25 \quad 29.868$ QPSK TC rate $2 / 3$ 81 28396 QPS K RS rate 12 06010700:03

ALN $105 \quad-25 \quad 29.868$ QPSK TC rate $2 / 38023396$ QPSK RS rate 12 06:010700:04

ALN 90 $25 \quad 29.868$ QPSK TC rate $2 / 3 \quad 8028396$ QRSK RS rate 12 06:01/0700:05

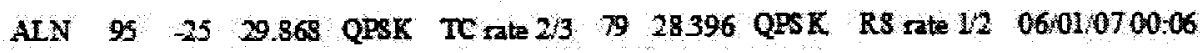




\subsection{Modern Congestion Control Technique}

\subsubsection{Literature review}

Congestion in an IP-based network happens when the network node is overwhelmed by packets. When the network is in a congestion state, packets are dropped in clusters. Lost packets result in failure to meet QoS requirements in the network. It could also lead to packet retransmissions, which costs extra network resources. The purpose of congestion control is to ensure network stability, throughput efficiency, and fair resource allocation [11]. Congestion control in the territorial IP network has been extensively studied for several decades. Existing network congestion control methods in literature cover a large range of techniques, including Additive Increase Multiplicative Decrease (AIMD) [12], source quench [13], slow start [14], schedule-based control [15], binary feedback [16], rate-based control [17], etc. The idea of the AIMD algorithm is used in traditional TCP window adjustments to achieve network congestion control. The algorithm achieves stability and fairness when the demand exceeds the channel's capacity [12]. The binary feedback method represents a network condition which uses a single bit. The system responds 1 when there is bandwidth available and 0 when bandwidths are saturated. However, the responsive behaviour of the network can hardly be completely described by a single bit. Rate control algorithms can limit traffic to a specific rate, but they cannot satisfy the QoS requirement on different types of traffic [11].

Recently, the Active Queue Management (AQM) has been proposed in order to support the SLA QoS requirements and attracted much attention to the research 
community. $\mathrm{AQM}$ is a packet dropping/mechanism for network router queue management. AQM mechanisms fall into two categories. The first is the Random Early Detection (RED) [19] and its variant, and the second is the Proportional Integral Derivative controllers (PID) [20]. RED algorithm was first proposed in the early 1990s, and it has been well developed. It has been widely deployed in today's IP routers, but it is often disabled by users due to its drawbacks, such as sensitivity to network configurations and parameter settings [21]. PID mechanisms emerge from control theories, and they have better performance in terms of end-to-end delay; however, it involves large queue length oscillation, which leads to a queuing time fluctuation.

In regards to high bandwidth-delay products, as the bandwidth and delay increase in the network, the TCP becomes inefficient and unstable [22]. Due to inherently large channel latency, congestion control in broadband satellite networks becomes important in committing QoS under inclement weather conditions. Broadband satellite networks have a larger round trip delay in comparison to traditional territorial IP networks; as a result, acknowledgement of TCP packets may be outdated when it comes back to the sender. For a round trip delay of $580 \mathrm{~ms}$, the network condition could change completely. The behaviour of a network is usually highly non-liner, time varying, and chaotic [23]. In addition, the congestion control of a broadband satellite network involves the weather conditions of the surrounding area surrounding the GT. Therefore, the congestion control of the broadband satellite network becomes indistinct. In recent years, some fuzzy logic based mechanisms have been proposed for congestion control in various territorial networks [24] [25] [26]. In this thesis, a fuzzy logic controlled rate-limited AQM scheme 
was proposed for the broadband satellite network in order to combat degraded quality of satellite channels. A RED algorithm was used as a queue management mechanism. A fuzzy-logic controller using current weather conditions dynamically tunes the RED parameters in order to overcome the drawbacks of the RED algorithm. The token bucket rate control algorithm controls the rate of incoming traffic to satellite network under different weather conditions. In addition, a priority path was created for QoS-sensitive applications such as VoIP service; this was done so that the services can be immutable under inclement weather conditions. In the congestion adaption system, the traffics are separated into high priority traffic and low priority traffic. They are distinguished using the Explicated Congestion Notification (ECN) bits in the IP header. ECN is an extension of IP which allow end-to-end congestion notification without dropping the packet. In this system, it was used as a priority identifier. The detail design is described in Section 3.3. The token bucket algorithm and RED algorithm are reviewed in the following section.

\subsubsection{Token Bucket Algorithm}

A token bucket algorithm is a flexible traffic shaping algorithm that limits the maximum traffic rate while allowing specific amounts of bursty traffic in the egress point [27]. In the algorithm, tokens are assigned a certain size, usually in bytes, and they are generated at a specific rate of $n$ tokens per $\Delta t$ second. Unused tokens will be stored in the bucket. If the number of the tokens in the token bucket exceeds the maximum bucket size $M$, newly arriving tokens will be dropped. The packet is transmitted if the tokens in the bucket can cover the length of the packet in bytes. Thus, the number of available tokens in the bucket 
determines how many packets can be transmitted. The maximum bucket size determines the maximum burst size. Unsent packets will be queued. The packets in the transmission queue may be transmitted, marked, or dropped depending on the length and policy of the transmission queue. Figure 2.4 illustrates the token bucket rate-control algorithm. In satellite communication, the satellite link will be degraded due to dynamic weather conditions. Thus, a token bucket algorithm is used to shape the traffic injected into the satellite network when the link capacity of satellite channel is reduced.

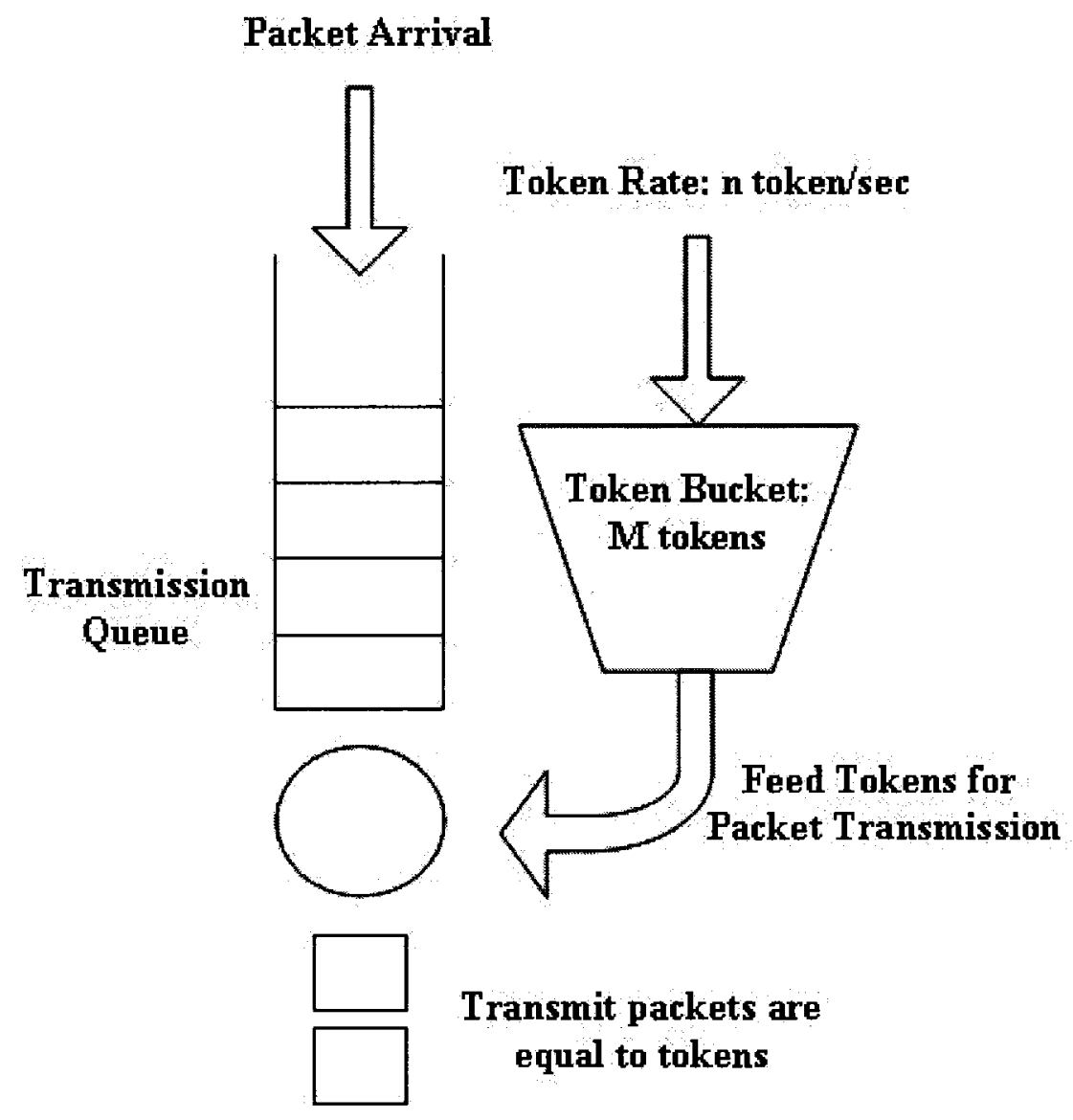

Figure 2.4: Functional diagram for token bucket algorithm [27] 


\subsubsection{Random Early Detect (RED) Algorithm}

RED algorithm is a congestion avoidance algorithm designed for packet-switch networks; this type of algorithm was first introduced in [19]. It is also a well-known Active Queue Management $(\mathrm{AQM})$ mechanism for router queue management. $\mathrm{AQM}$ is proposed in order to avoid network congestion and to support QoS commitment. It targets at reducing the average queue length, and thereby decreasing the end-to-end packet delay and ensuring efficient network resource utilization. The main objective of RED is to avoid network congestion by controlling the average queue size in a reasonable range. RED maintains an average queue (avg) in packets using exponential weighted moving average (EWMA). The average queue size is compared to the minimum $\left(T_{\min }\right)$ and maximum $\left(T_{\max }\right)$ queue thresholds. Newly arrived packets are processed according to the average queue size. When the average queue size is less than the minimum queue threshold, no packets are dropped. If the average queue length is larger than the maximum threshold, every arriving packet will be dropped. When the average queue size falls in the range between the maximum threshold and the minimum threshold, drop the arriving packet with $P_{b}$, which is calculated based on the maximum dropping probability $P_{\max }$, current average queue length, and the average queue thresholds. The flow chart of the RED algorithm is shown in Figure 2.5 below. 


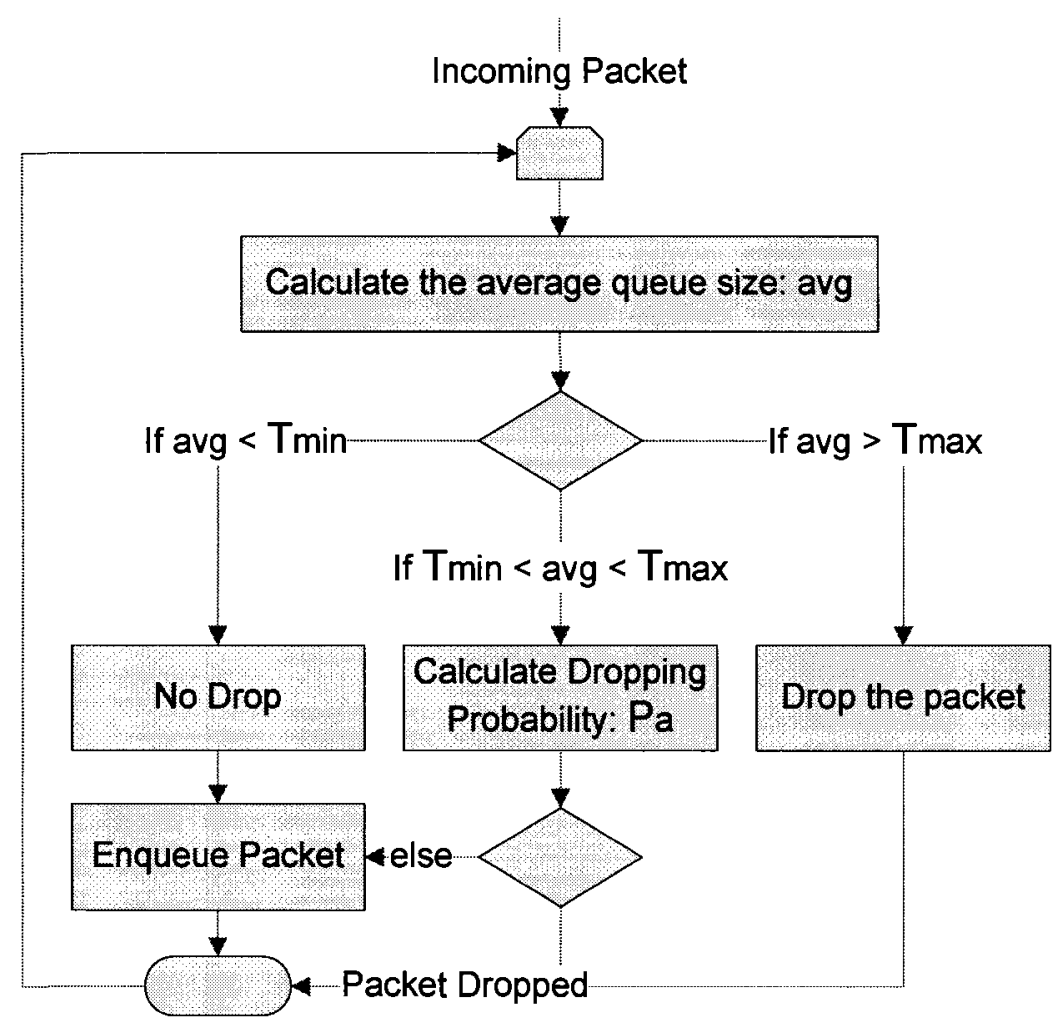

Figure 2.5: Flowchart for RED algorithm [19]

The average queue size is calculated upon each packet arrival. When the queue is non-empty, the average queue size is calculated as follow:

$$
a v g=\left(1-w_{q}\right) \times a v g+w_{q} \times q
$$

where $w_{q}$ is the queue weight and $\mathrm{q}$ is the current queue size. If the queue is empty, the average queue size is calculated according to:

$$
a v g=\left(1-w_{q}\right)^{m} \times a v g
$$

where $m$ is the number of packets arrived at the empty queue during the empty queue period. 
When the average queue size varies between $T_{\min }$ and $T_{\max }$, the incoming packet is dropped with probability $P_{b}$, which varies from 0 to $P_{\max } . P_{b}$ can be calculated by:

$$
P_{b}=P_{\max } \times\left(\operatorname{avg}-T_{\min }\right) /\left(T_{\max }-T_{\min }\right)
$$

The RED algorithm avoids the network congestion at early stages by controlling the average queue size. It keeps the average queue size low while allowing occasional bursty traffic. The study in [28] showed that the performance of the RED algorithm relies on four fixed parameters: $w_{q}, P_{\max }, T_{\min }$ and $T_{\max }$. Since the network configuration and network state changes, the performance is very sensitive to parameter settings. However, RED was proven to be stable, but requires parameter tuning to reach optimal channel efficiency [29]. The variants of the RED algorithms designed in [30], [31], and [32] show the possibility of using a fuzzy logic controller to tune the RED parameters for better system performance. In this thesis, the fuzzy logic concept was applied to adaptively set the RED parameters; this was done so that congestion in the IP-based satellite network can be controlled more effectively. The original frame work was published in [33]. 


\subsection{Satellite Terminal Fault Diagnosis System}

\subsubsection{Literature Review}

Satellite GT is the gateway for a local customer to connect to the satellite network. As a result, the availability of a satellite ground terminal is related to the service availability to the satellite network subscribers. The terminal fault diagnosis system helps the satellite service provider identify the operating condition of the remote GTs. A correct diagnosis can help the satellite service provider recover faulty GT efficiently. In literature, efforts have been made towards the fault diagnosis of satellite GT and satellite communication systems. Adding an additional subsystem in satellite GT hardware can diagnose and isolate hardware faults effectively [34]. The Bayesian Network [35] and graphical modeling technique [36] are also used to diagnose the failure of satellite GT at system level. Artificial intelligence techniques such as neural networks [37] and expert system [38] [39] have been widely applied to fault detection and fault isolation for satellite communication systems. However, previous works mainly focused on hardware fault diagnosis and system level fault diagnosis. These methods are not capable of detecting LoS related terminal faults. A recent publication proposed a wavelet-based approach to identifying the LoS impairment fault, power fault, and hardware faults [40]. The waveletbased SNR analysis identified the correlation between terminal faults and received SNR. However, the diagnosis accuracy of a wavelet-based method is inconsistent for different types of faults. In this thesis, a knowledge-based TFD system was proposed in order to diagnose the operating conditions of a satellite ground terminal. In the knowledge-based 
TFD system, a fuzzy inference system is used in order to make decisions based on a predefined rule set that were translated from human expertise. Appendix $\mathrm{C}$ explains the principle of the fuzzy logic theory and the Fuzzy Inference System (FIS). 


\section{Chapter 3}

\section{Congestion Adaptation System}

\subsection{Overview}

In a broadband satellite network, the channel capacity degrades severely as the rain/snow-fall rate increases. The objective of the congestion adaptation system is to maintain QoS for high priority traffic and to avoid network congestion under dynamic weather conditions. In traditional territorial IP networks, channel capacity is fixed; therefore, bursty traffic or enormous user traffic causes network congestion. Therefore, network congestion in a traditional territorial network can easily be controlled with AQM mechanisms. Furthermore, the optimization of $\mathrm{AQM}$ mechanisms is also straightforward, because only local information, such as local queue length or packet incoming rate are needed. In the congestion control of a broadband satellite network, network congestion is mainly caused by external events, such as weather change. Moreover, inclement weather conditions reduce channel capacity severely, which makes congestion control in broadband satellite networks more difficult. 
When designing the congestion adaption system, there were two main challenges. First, since the network did not have the ability to react when weather conditions changed, congestion was caused by an increased rain/snow-fall rate. Although existing AQM mechanisms can drop packets at the network node so as to avoid congestion in an early stage, unfortunately, they drop packets blindly. Second, the channel capacity reduces temporarily under rain/snow-fall. When channel capacity was reduced, meeting QoS requirements for high priority traffic became difficult and only achievable at the expense of low priority traffic.

In this thesis, a fuzzy-logic controlled, RED-based, and rate-control enabled congestion adaption system was proposed in order to overcome the problems in broadband satellite communication. The RED algorithm was used in order to manage transmission queue in the system, because the RED algorithm can effectively drop/mark packets so as to avoid network congestion. In order to achieve steady performance, the RED parameters need to be tuned according to different network configurations. In addition, the channel capacity varies as the weather conditions change. As a result, it is important to tune the RED parameters and rate-control parameters based on the rain/snow-fall rate.

Since the rain/snow-fall rate is external information, there is no direct relation between snow/rain-fall rate and system parameters. Tuning the parameters relies heavily on experienced network engineers. Instead of using a set of IF-THEN rules to determine system parameters, the fuzzy-logic controller was used in order to determine the system parameters. There are two advantages from using the fuzzy logic controller. First, the 
fuzzy logic can reproduce human knowledge in a systematic manner. Second, the knowledge base of the fuzzy logic control is upgradeable. Rules can be added to the knowledge base when any network configurations change.

The proposed congestion adaption system brought three new concepts to congestion control in the territorial IP network. First, the congestion adaptation system took external information into account. Second, proper membership functions were used in order to represent the rain/snow-fall rate and system parameters. Third, a unique rule set is defined in order to represent the knowledge of experienced network engineers.

The next section will describe the architecture of the proposed congestion adaption system. The remaining sections of this chapter will explain how the rate-control algorithm, RED algorithm, and fuzzy logic controller are integrated into the congestion adaptation system. 


\subsection{System Architecture}

The congestion adaptation system developed in this thesis is deployed at the satellite hub and GTs. The system regulates the incoming traffic entering the satellite network. Figure 3.1 shows the system software stack. The traffic that goes through the gateway will be captured and processed by the congestion adaptation system and then pushed back into the network. The congestion adaptation system used an AQM scheme, and it was incorporated with a fuzzy logic controller in order to tune the queue thresholds and transmission rates. The system enforces all QoS related processing on the user data traffic flow entering the satellite network prior to transmission over the air. The congestion adaptation system will be implemented in both the satellite hub and in remote GTs.

The system architecture of the congestion adaptation system is illustrated in Figure 3.2. The congestion control of broadband satellite network is mainly dependent on three primary functions of the congestion adaptation system: Pack Conditioning, QoS Arbitrator, and Traffic Shaper. The Packet Conditioning function is responsible for classifying the incoming packets under either priority or non-priority based on the application port number. Every IP packet that is captured by the congestion adaptation system will be processed by the Packet Conditioning function first; here, the ECN bits in the Type of Service (ToS) field in the packet header will be marked in order to identify the priority level. Then, the packet will be passed onto the Traffic Shaper, where the QoS policy is enforced before entering the satellite network. 
Based on real-time weather information, the QoS Arbitrator will wake up its embedded fuzzy-logic controller in order to return new RED and rate-control parameters to the Traffic Shaper. A detailed explanation of data flow at the traffic shaper is listed below:

1. Upon each packet arrival, the Traffic Shaper will place it into either a priority queue or non-priority queue depending on its ECN bits.

2. According to the current status of the queue, the Traffic Shaper will decide whether the packet should be sent, queued for transmission, or dropped.

3. Finally, the Traffic Shaper sends out packets at a rate no greater than the maximum rate that is determined by the Token Bucket Algorithm.

Packets leaving the congestion adaptation system will enter the satellite network. The congestion adaptation system enforces the QoS policy and regulates the traffic load that flows into the satellite network; hence, it protects the satellite network from congestion. The detailed designs of the three primary functions of the congestion adaptation system are explained in the following sections.

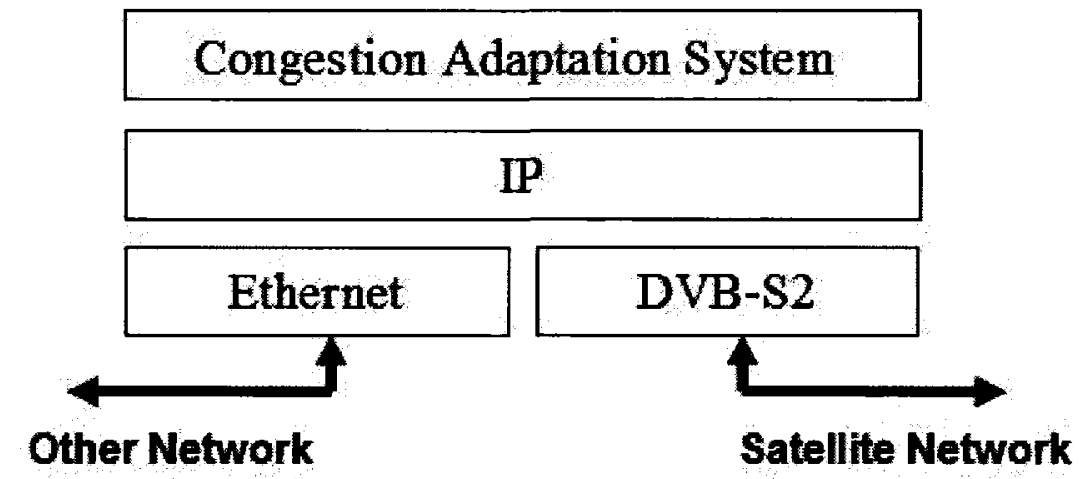

Figure 3.1: Congestion adaptation system software stack 


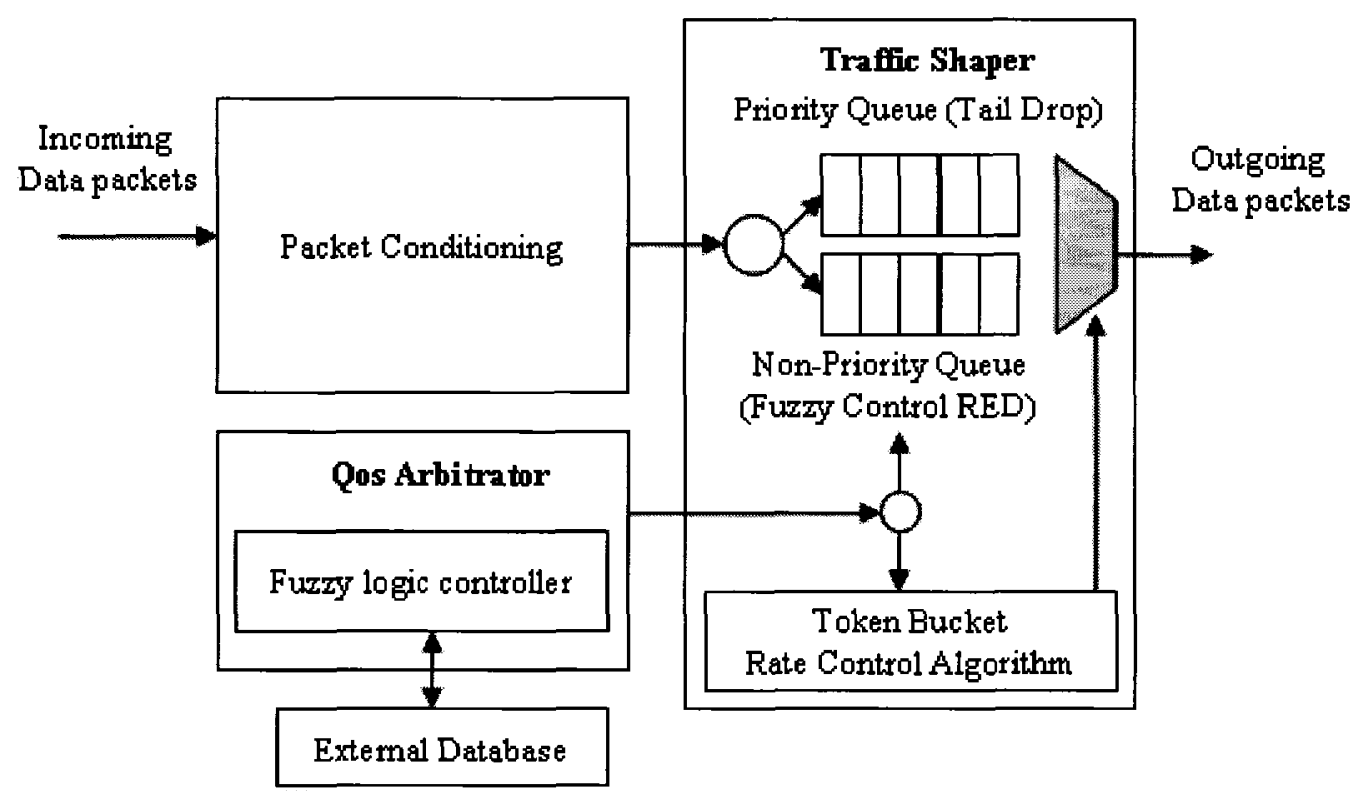

Figure 3.2: System architecture of congestion adaptation system 


\subsection{Packet Conditioning}

One of the main objectives of the congestion adaptation system is to improve the performance of the priority traffic stream. In the congestion adaptation system, ECN bits in ToS field were used to indicate the priority level of the packets. The ToS field in the IP header is defined in [45]. It has 8 bits and it is located at the second octet of the IP header. Figure 3.3 shows the ToS field in detail. The first three bits are precedence bits. Eight different values in this field stand for eight different priority levels. Their possible value and meanings are given in Table 3.1. The next four bits are the ToS bits. Each of the bits indicates a feature. Table 3.2 explains the usage of these bites. Setting all the ToS bits to 1 will speed up the packet flow. The last bit is the checking bit, which is always 0 . However, this feature is rarely used in today's IP network. ECN bits were proposed in [46] and finalized in [47]. ECN bits are extensions of the ToS bits. They use the last two bits of the ToS field. 00 in the ECN bits mean there is no explicate notice of congestion. 01 means the ECN is enabled. In the congestion adaption system, 00 in the ECN field means low priority traffic and 01 in ECN bits represents high priority traffic.

The Packet Conditioning function of the congestion adaptation system determines the priority of packets depending on its application port number. Upon the arrival of each packet, the function looks up the application port number of the packet. If the port number matches with a specific application port in our priority traffic category, the function will change the ECN bits to 01. Classifying the packets enabled the Traffic Shaper of the congestion adaptation system to enhance QoS policy on specific 
applications over others. The design of the Traffic Shaper and the QoS Arbitrator are discussed in the following sections.

\begin{tabular}{|l|l|l|l|l|l|}
\hline \multicolumn{1}{|c}{4} & 5 & 6 & 7 & 8 \\
\hline Precedence & Delay & Throughput & Reliability & Cost & MBZ \\
\hline
\end{tabular}

Figure 3.3: ToS fields in the IP header

Table 3.1: Possible values of precedence bits and their meanings

\begin{tabular}{|c|c|}
\hline 000 & Routine \\
\hline 001 & Priority \\
\hline 010 & Immediate \\
\hline 011 & Flash \\
\hline 100 & Flash Override \\
\hline 101 & Critical \\
\hline 110 & Internetwork Control \\
\hline 111 & Network Control \\
\hline
\end{tabular}

Table 3.2: Possible values of other ToS bits and their meanings

\begin{tabular}{|c|c|c|}
\hline & 0 & 1 \\
\hline Delay & Normal & Low \\
\hline Throughput & Normal & High \\
\hline Reliability & Normal & High \\
\hline Cost & Normal & low \\
\hline MBZ & \multicolumn{2}{|c|}{ Must Be Zero } \\
\hline
\end{tabular}




\subsection{Functionality of Traffic Shaper}

When the remote site is experiencing rain/snow-fall, the signal strength of the down link may be degraded severely. Maintaining the QoS policy in these situations became very difficult. The Traffic Shaper is designed to support the QoS for high priority traffic under inclement weather conditions, at the expense of low priority traffic.

As shown in Figure 3.2, the incoming traffic is categorized to either a priority queue or a non-priority queue, according to the ToS bits in the IP header. The priority queue used a drop tail queue management algorithm. It holds packets up to its buffer size and drops the incoming packets when the queue is full. In this scheme, when enough tokens are available, the packets from priority will be transmitted first regardless of the queue condition in the non-priority queue. The packets from non-priority are transmitted only if the priority queue is empty. This design enhanced the performance of the priority traffic; however, the trade off is packets queuing up at the non-priority queue.

In order to combat the network congestion that may occur at the non-priority queue, a RED algorithm was used in order to prevent network congestion at an early stage. As discussed in Section 2.2, the performance of the RED algorithm is sensitive to parameter setting. Although it may improve the performance, it is more difficult to choose parameters dynamically al. A novel fuzzy-logic based scheme was proposed to dynamically set the parameters for varying weather conditions. Furthermore, a different traffic shaping rate will be applied for different rain/snow-fall rates recorded at the remote site. The details are shown in the next section. 


\subsection{Design of QoS Arbitrator}

The purpose of the QoS Arbitrator is to tune the system parameters of the congestion adaptation system. It uses a fuzzy-logic controller to determine RED parameters and traffic shaping rate based on rain/snow-fall rate. The following section describes the design of the fuzzy-logic controller for QoS Arbitrator in detail.

\subsubsection{Mapping Input/Output Variable into Fuzzy Sets}

The QoS Arbitrator is designed to tune system parameters according to the snow/rain-fall rate. The system parameters that need to be tuned are maximum transmission rate for token bucket algorithm, maximum queue threshold, and maximum dropping probability for the RED algorithm. Because Telesat emphasized that the amount of traffic entering the satellite network needs to be limited when channel capacity decreases, the maximum transmission rate for the token bucket algorithm needs to be tuned according to current weather conditions. As investigated in Section 2.2, the RED parameters need to be tuned to achieve steady performance. In this broadband satellite network, channel capacity reduces as rain/snow-fall rate increases, so the system needs to tune the RED parameters accordingly in order to obtain steady performance. Therefore, one input and three outputs are defined for the fuzzy logic controller.

The triangle shaped membership function was used to map all input and output variables. Triangle membership functions are commonly used when applying fuzzy logic control in telecommunication [30] [31] [32]. In literature, when mapping variables into fuzzy sets, all the fuzzy sets share the same shape of membership functions and have the same range. Moreover, the centers of fuzzy sets are usually evenly distributed within the 
range of a variable. Suppose the input is snow/rain-fall rate and the range of the snow/rain-fall rate is 0 to 60 millimetres per hour, and the snow/rain-fall rate needs to be mapped into five fuzzy sets, which are Sunny, Drizzle, Light Rain, Heavy Rain, and Thunder Storm. Figure 3.4 illustrates the membership functions of the fuzzy sets using the traditional mapping method. Each fuzzy set has the same shape, and their centers are evenly distributed over the range of the rain/snow-fall rate. This method works well with most network parameters such as the transmission rate, packet receiving rate, or packet dropping rate, where their fuzzy sets can be evenly distributed and still make sense. However, the congestion adaptation system needs to deal with the rain/snow-fall rate in the broadband satellite network. If the fuzzy sets are evenly distribute within the range of rain/snow-fall rate, the linguistic meaning of the fuzzy sets will be violated. As shown in Figure 3.4, the fuzzy set Drizzle has the range from 0 to 30 millimetres per hour, and it is centered on 15 millimetres per hour. The linguistic meaning of Drizzle is very light rain. As a result, the assignment of the fuzzy set Drizzle oversteps the bounds of drizzle. Therefore, the use of the traditional mapping method on the rain/snow-fall rate will misrepresent the fuzzy sets.

The customized membership functions for rain/snow-fall rate are shown in Figure 3.5. The choice of the membership functions is based on historical data and empirical analysis. 


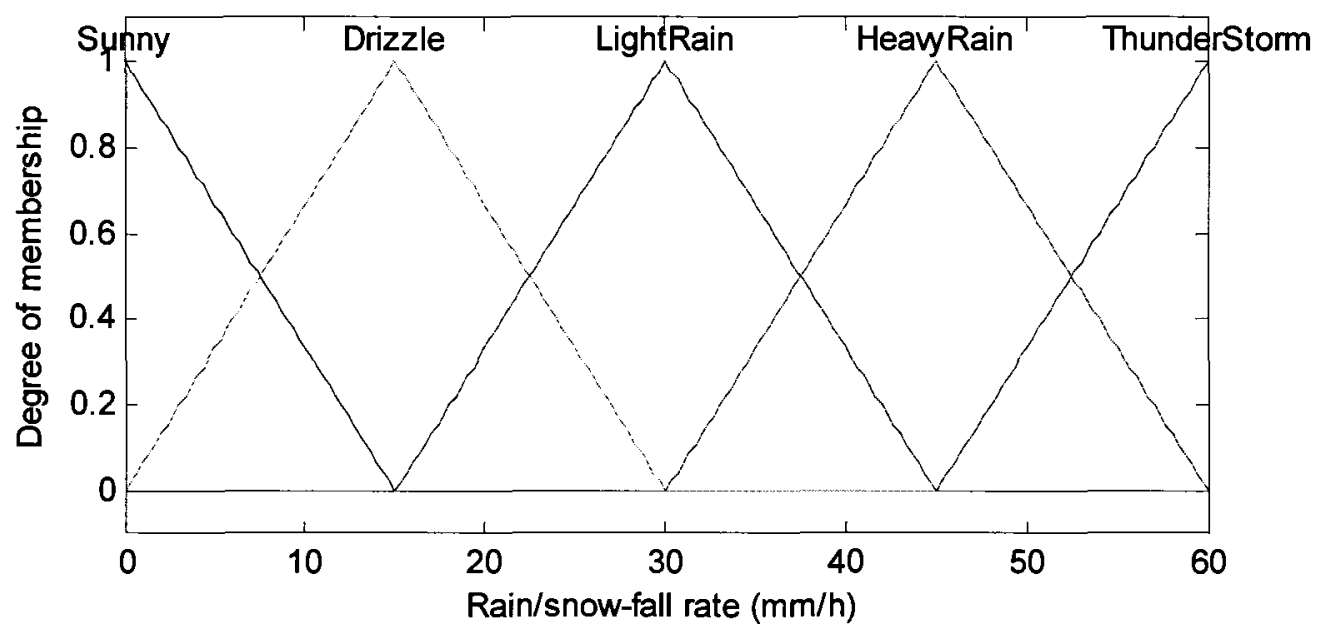

Figure 3.4: Membership functions for rain/snow-fall rate using traditional mapping method

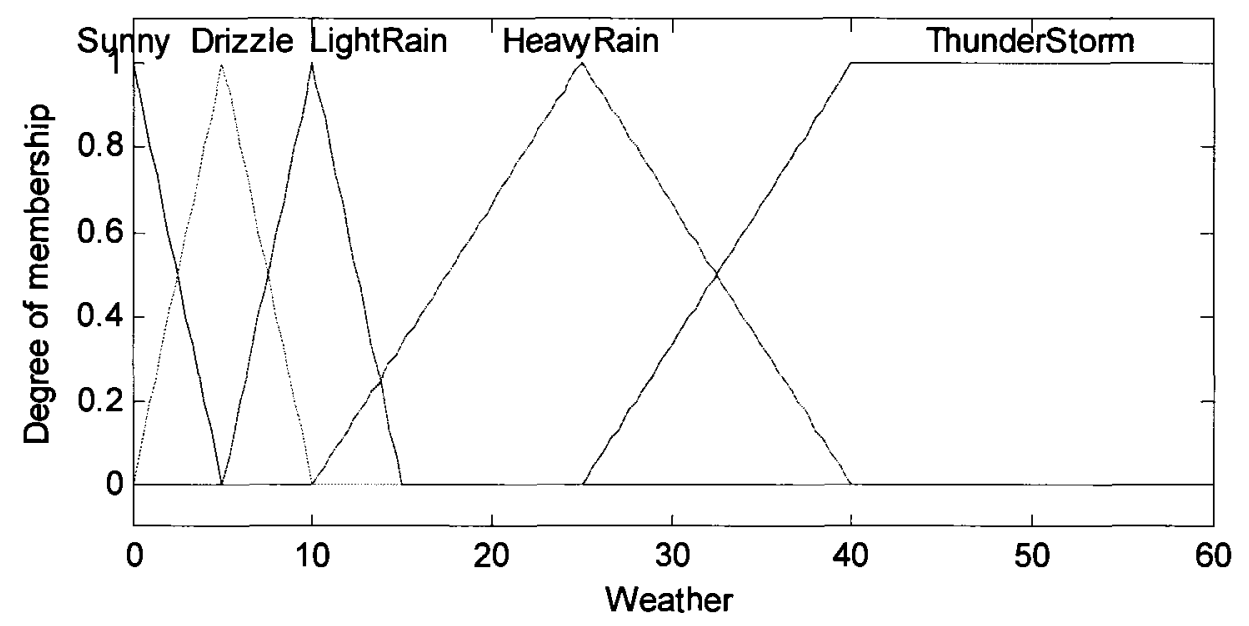

Figure 3.5: Membership functions for rain/snow-fall rate

The three outputs of fuzzy-logic controller are maximum transmission rate of the token bucket algorithm, maximum queue threshold, and maximum dropping probability for the RED algorithm. In order to match the fuzzy sets of input to the fuzzy sets of output uniquely, five fuzzy sets were created for each output variable; these sets include 
Very Low, Low, Medium, High and Very High. A traditional mapping method was used when mapping the output variables into fuzzy sets. The assignment of membership functions are shown in Figure 3.6 to Figure 3.8.

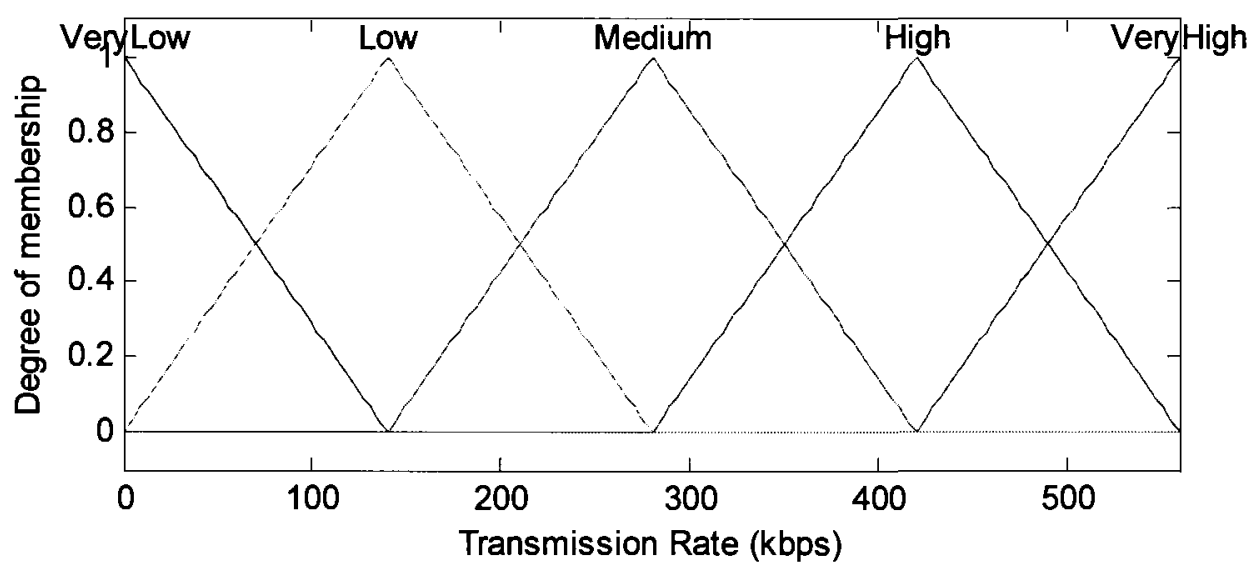

Figure 3.6: Membership functions for maximum transmission rate

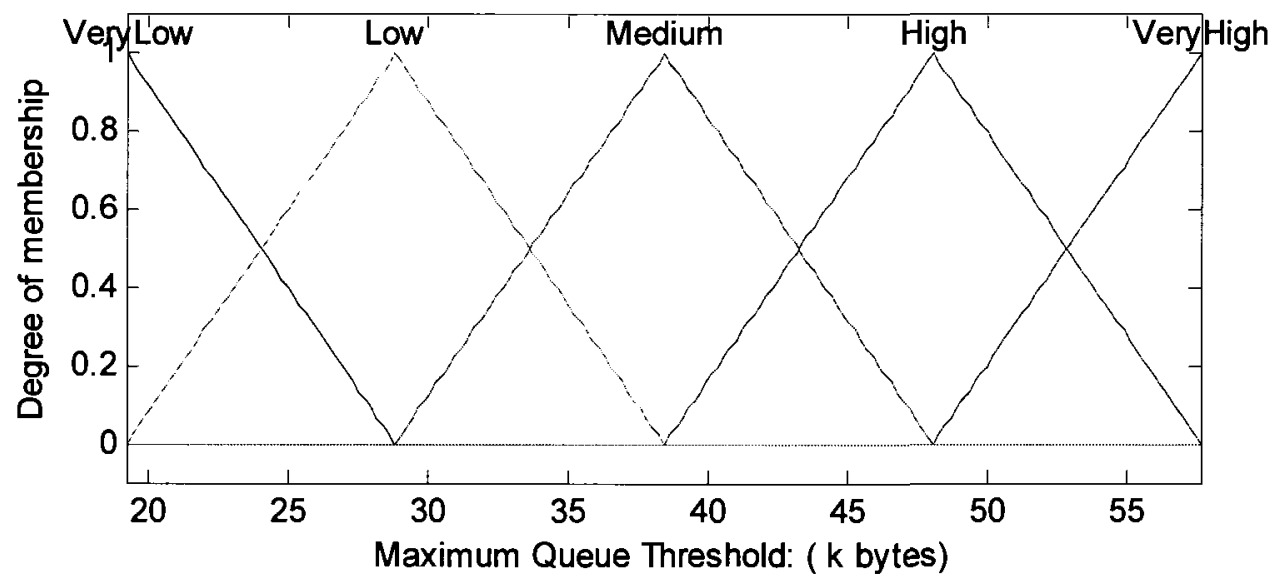

Figure 3.7: Membership functions for maximum queue threshold 


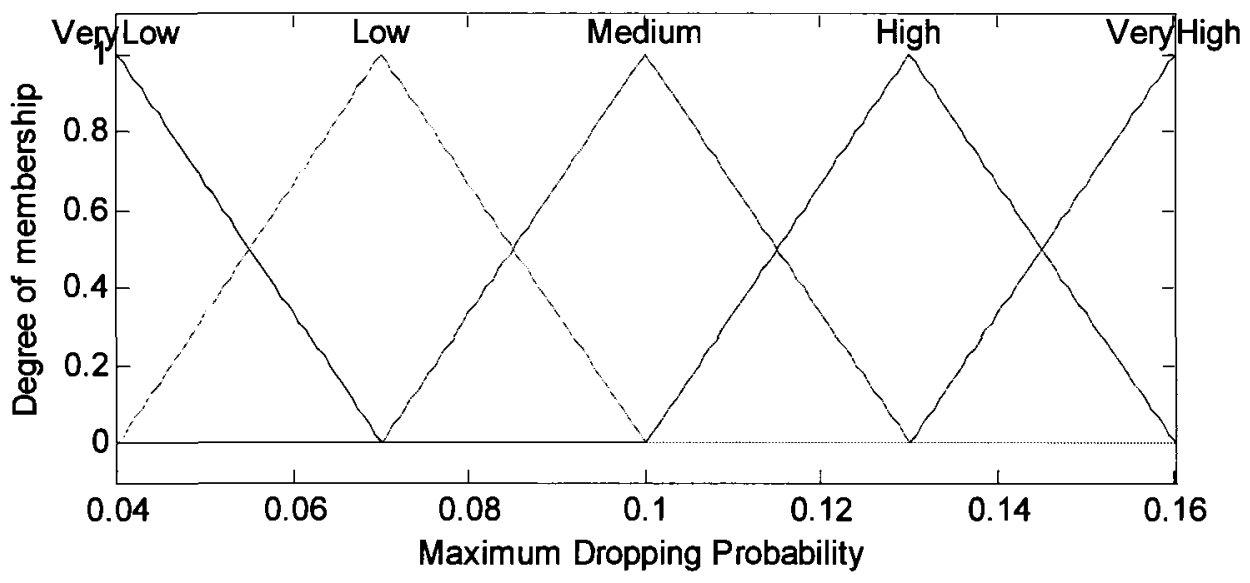

Figure 3.8: Membership functions for maximum dropping probability

\subsubsection{Constructing Knowledge Base}

According to an empirical analysis of numerous simulations and testing results, a set of rules were created in order to represent the knowledge of experienced network engineers. The rule set for the QoS Arbitrator is listed in Table 3.3. For example, when the rain/snow-fall rate is low, the maximum transmission rate for the token bucket algorithm should be close to the channel capacity. If the rain/snow-fall rate is high, then the maximum queue threshold should be set to low and the maximum dropping probability should be set to high in order to drop packets earlier in order to prevent network congestion. Figure 3.9 shows the output of the maximum transmission rate for the token bucket algorithm as the rain/snow-fall rate changes from 0 to 60 millimetres per hour. Figure 3.10 and Figure 3.11 show the change in maximum queue threshold and maximum dropping probability for the RED algorithm respectively. 
Table 3.3: Rule set for QoS Arbitrator

\begin{tabular}{|c|c|c|c|c|}
\hline & Antecedent & \multicolumn{3}{|c|}{ Consequent } \\
\cline { 2 - 5 } & Weather condition & $\begin{array}{c}\text { Maximum } \\
\text { Transmission } \\
\text { Rate }\end{array}$ & $\begin{array}{c}\text { Maximum } \\
\text { Queue } \\
\text { Threshold }\end{array}$ & $\begin{array}{c}\text { Maximum } \\
\text { Dropping } \\
\text { Probability }\end{array}$ \\
\hline $\mathbf{1}$ & Sunny & Very High & Very High & Very Low \\
\hline $\mathbf{2}$ & Drizzle & High & High & Low \\
\hline $\mathbf{3}$ & Light Rain & Medium & Medium & Medium \\
\hline $\mathbf{4}$ & Heavy Rain & Low & Low & High \\
\hline $\mathbf{5}$ & Thunder Storm & Very Low & Very Low & Very High \\
\hline
\end{tabular}

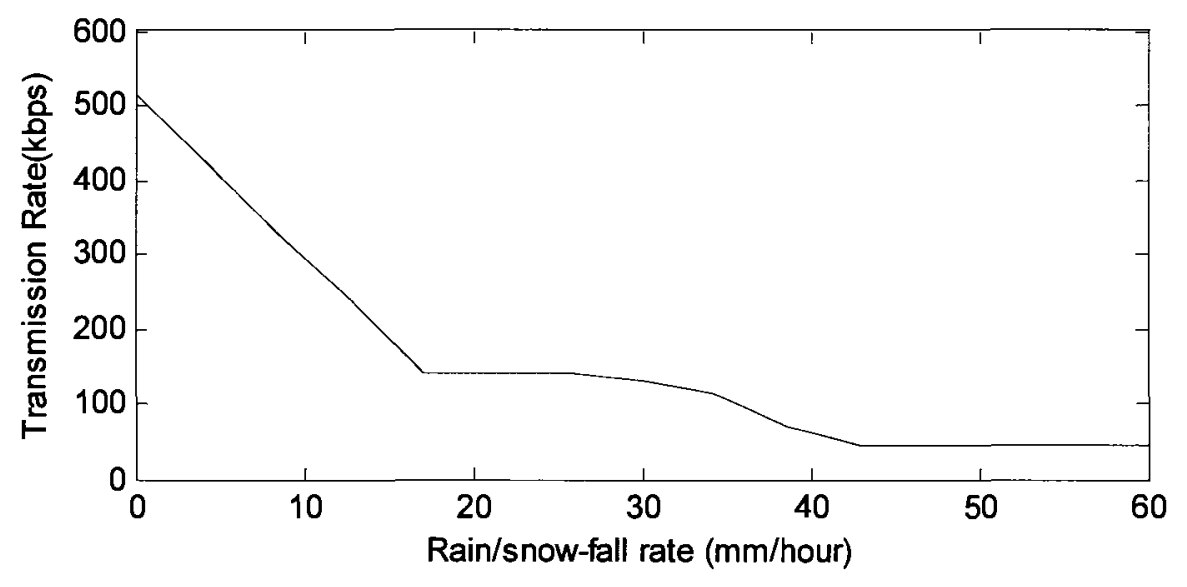

Figure 3.9: Output range of maximum transmission rate

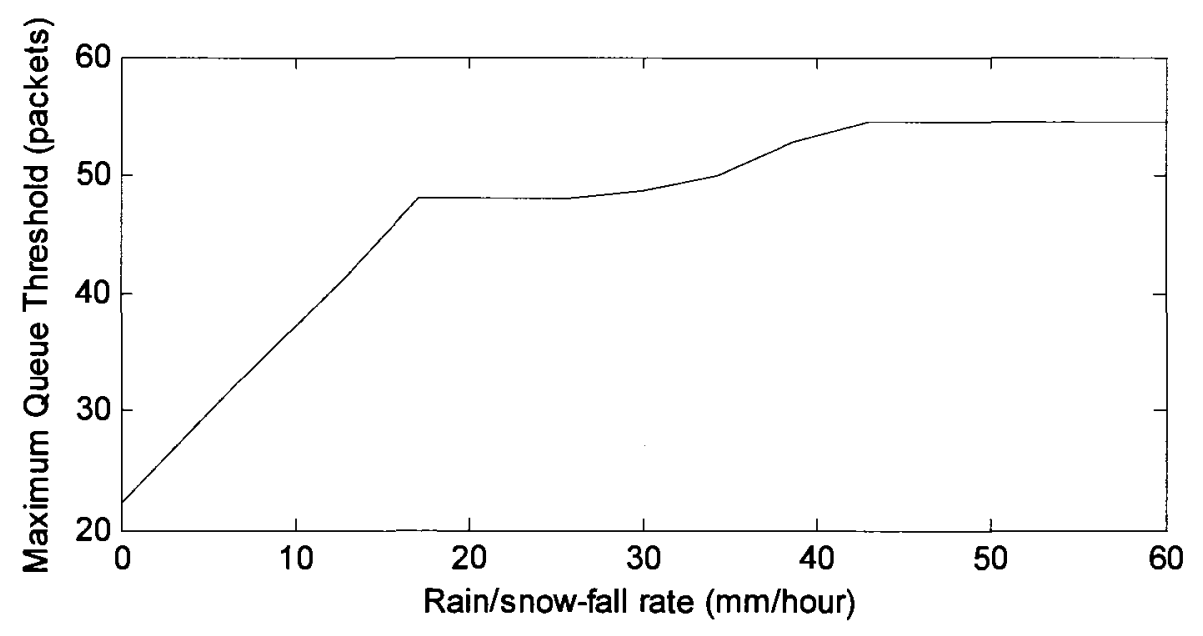


Figure 3.10: Output range of maximum queue threshold

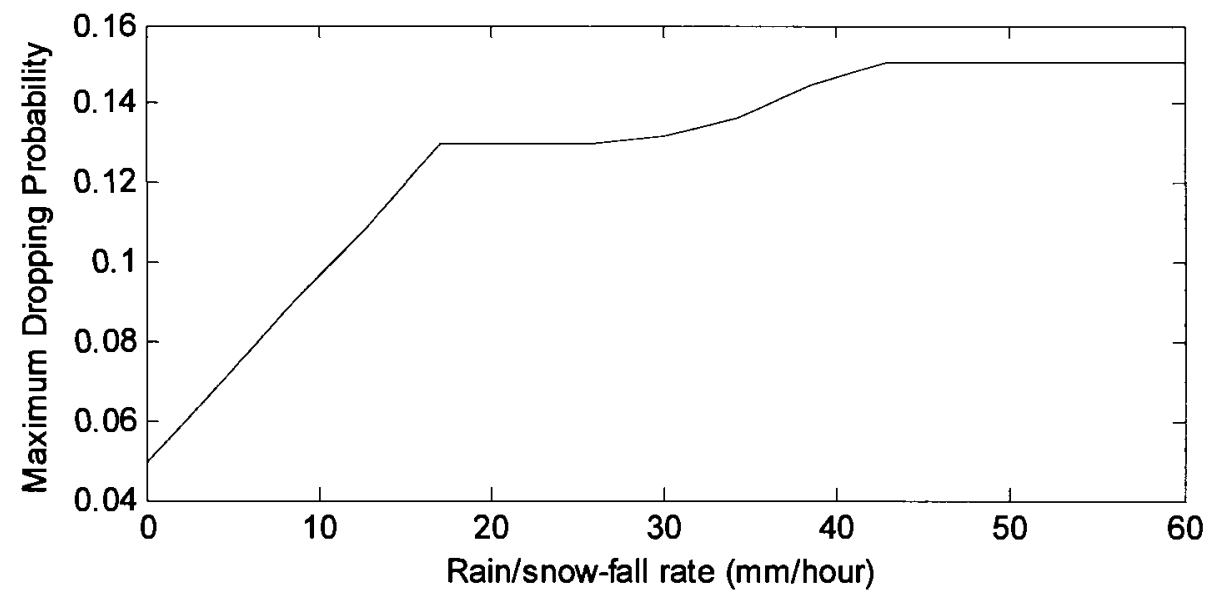

Figure 3.11: Output range of maximum dropping probability

For each fuzzy inference process, the fuzzy-logic controller maps the rain/snow-fall rate to a given fuzzy set and obtains a linguistic variable with a degree of truth. Then, the fuzzy-logic controller looks up the rules from the knowledge base in order to decide the union of fuzzy sets for the output parameters. Numerical outputs can be obtained by defuzzifying the union of fuzzy sets. The following example explains how the output parameters are determined when the current snow/rain-fall rate is given.

Suppose current rain/snow-fall rate is 8 millimetres per hour. According to Figure 3.5, the rain/snow-fall rate is mapped to linguistic variable drizzle and light rain with different degrees of truth. The graphical expressions of the linguistic variables are displayed on the left hand side of Figure 3.12. Because linguistic variable Drizzle and Light Rain matched the antecedents of rule (2) and rule (3) in the knowledge base, rule (2) and rule (3) were executed. The consequent of rule (2) and (3) were combined into union of fuzzy sets, and they are illustrated in Figure 3.12. The outputs of the system parameters were obtained by defuzzifying the union of fuzzy sets. 


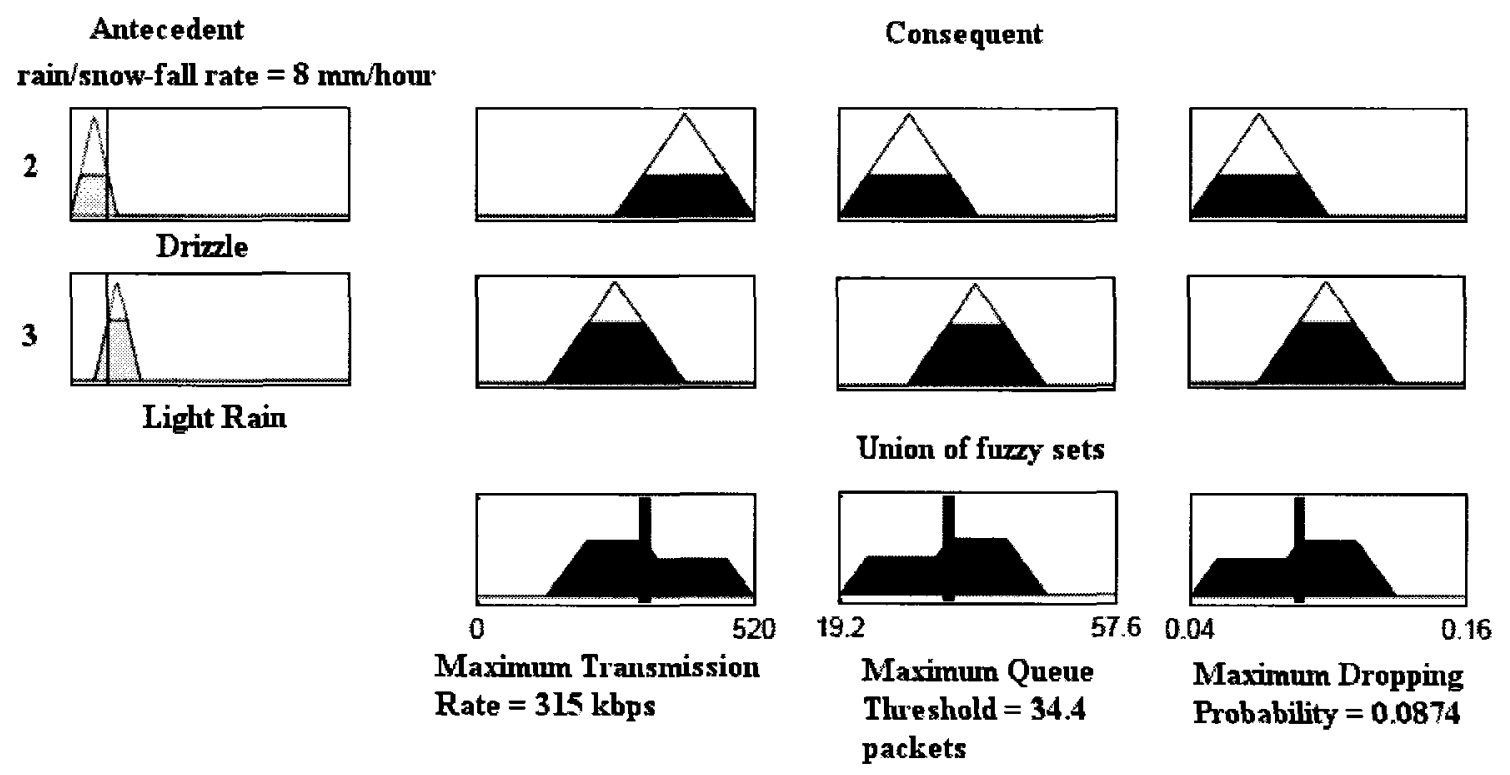

Figure 3.12: Determining QoS parameters using fuzzy logic controller

The range of the system parameters changes when the network configurations change. The settings discussed in this section are for the return link channel, because current tests are only performed using the return link channel in the test-bed network. The settings for the forward link channel need to be tuned after testing the forward link channel. In order to achieve the best performance, the membership functions and the rules in the knowledge base need to be tuned based on the field test results.

In conclusion, this chapter described the design of the proposed congestion adaption system. This design introduced the concept of using external information such as rain/snow-fall rate in congestion control of broadband satellite networks. There were no existing techniques in literature regarding how to use the rain/snow-fall rate in congestion control. This thesis explains how to develop a fuzzy-logic based knowledge system that tunes system parameters based on the knowledge of experienced network engineers. A 
set of membership functions were also uniquely defined in order to represent the rain/snow-fall rate. Unique rules were created so as to represent the knowledge of the experienced network engineers. The congestion adaptation system has been successfully implemented and tested in a broadband satellite network test-bed. Test results are shown in Chapter 5. 


\section{Chapter 4}

\section{Knowledge-based Terminal Fault Diagnosis System}

\subsection{Overview}

In a broadband satellite network, once GTs are deployed in remote sites, the satellite service provider relies on RF data that has been measured at the GTs in order to monitor the operating condition of the GTs. Properly diagnosing the current operating conditions helps the satellite service provider to improve the reliability of that GT. For example, if the satellite service provider notices a deteriorating performance of a GT in an early stage and provides proper instruction to the customer to fix the problem, then both the satellite service provider and the customer will benefit from the correct diagnosis. Currently, satellite operators diagnose the operating conditions of a GT by analyzing the historical forward link SNR (FLS). The diagnosis process and diagnosis results are highly dependent on the knowledge and the availability of the experienced satellite operators. There were no existing methods in past literature about diagnosing operating conditions of satellite GTs. The objective of a knowledge-based TFD system is to automate the diagnosis process based on the knowledge of experienced satellite operators. 
In this thesis, the Fuzzy Inference System (FIS) was used in order to construct a knowledge base that can reproduce the fault diagnosis process using expert knowledge. FIS is based on fuzzy logic. It contains a set of rules that follow expert thinking patterns. There are two great advantages for using FIS to construct a knowledge base. First, the FIS is constructed using a set of rules translated from experienced satellite operators. The rules can be written in plain language that can be understood right away by experts. Second, FIS has the ability to learn by adding new rules. This allows satellite service providers to upgrade the knowledge base after they learn from experience.

When designing a knowledge-based system, expert knowledge needs to be explicitly defined. According to a comprehensive analysis of historical FLS from different sites and the help from experienced satellite operators, the operating conditions of the ground terminal are classified into five phenomena, which are NORMAL, UNUSUAL, POWER OFF, CRITICAL, and FLAPPING. The details are shown in Section 4.3. Moreover, the thinking patterns of experienced satellite operators were summarized into four indicators and a SNR trending algorithm was designed in order to generate these indicators. The SNR trending algorithm is responsible for providing the four indicators to FIS by processing historical FLS from targeted GT. The detailed design of SNR trending algorithm is given in Section 4.4.

According to recommendations from experienced satellite operators, POWER OFF, CRITICAL, and FLAPPING conditions have strong graphical patterns. Thus, it is good to use a knowledge-based method to diagnose these phenomena. On the other hand, there is no clear decision boundary between the NORMAL and UNUSUAL condition. When 
making decisions according to a selected boundary using expert knowledge, the decision will be biased, and the boundary may not be suitable for every GT. As a result, the Maximum Likelihood method was used in order to determine whether a GT is operating under the NORMAL or UNUSUAL condition. The Maximum Likelihood method is a statistical tool that is used to make decisions in noisy environments. Section 4.5 below discusses how the Maximum likelihood method is implemented.

The knowledge-based TFD system proposed in this thesis introduces four new concepts into diagnosing operating condition of the satellite GT. First, the system identifies the operating conditions of the satellite GT using the FLS pattern. Second, the SNR trending algorithm is capable of generating expert thinking patterns. Third, the knowledge-based fault diagnosis system can diagnoses the operating condition of the satellite GT using both the Maximum Likelihood method and expert knowledge. Fourth, the system can properly reproduce the knowledge of experienced satellite operators.

Section 4.2 below briefly describes the system architecture of a knowledge-based TFD system. The rest of this chapter discusses the design of the SNR trending algorithm, Maximum Likelihood method, and knowledge base. 


\subsection{System Architecture}

The knowledge-based TFD System uses FLS measured at each GT in order to decide the operating condition of each GT. The system architecture of the knowledge-based TFD system is illustrated in Figure 4.1. The SNR trending algorithm, Maximum Likelihood decision method, and Fuzzy Inference System (FIS) are three key elements in the knowledge-based TFD system.

The SNR trending algorithm processes a bulk of RF samples together in order to generate four indicators that describe the trend of the FLS. The four indicators generated by the SNR trending algorithm are Terminal State (TS), Zero Count (ZC), Fault Indicator (FI), and Critical Indicator (CI). Section 4.4 describes how the indicators are generated and explains their significance and value.

The Maximum Likelihood method and FIS are the decision making units of the TFD system. The Maximum Likelihood method is used to determine whether a GT is operating under the NORMAL or UNUSUAL condition. According to our analysis, when GT is operating under the NORMAL and UNUSUAL condition, the value of TS follows Normal distribution with different means and variances. The Maximum Likelihood method is a statistical method that makes a decision based on observed values under noisy environmental conditions. Therefore, the Maximum Likelihood method is chosen in order to determine whether a GT is operating under the NORMAL or UNUSUAL condition, with the help of TS.

FIS uses rule sets that are translated from experienced satellite operators in order to determine the likelihood of POWER OFF, CRITICAL, and FLAPPING conditions. 
Because the decision on POWER OFF, CRITICAL, and FLAPPING conditions have strong FLS patterns, therefore, FIS is chosen to make empirical decisions. In FIS, knowledge is translated into rules and stored in the knowledge base. When the input matches the antecedent of a rule in the knowledge base, the rule will be executed. Also, many rules can be executed in one process. Thus, all uncertainties and possibilities are taken into account when making a decision.

The outputs from the Maximum Likelihood method and FIS are raw outputs. In order to correctly identify the operating condition of GT, only one condition will be generated for each SP. The decision constraint is designed to minimize false alarms. The detailed design is given in section 4.8 .

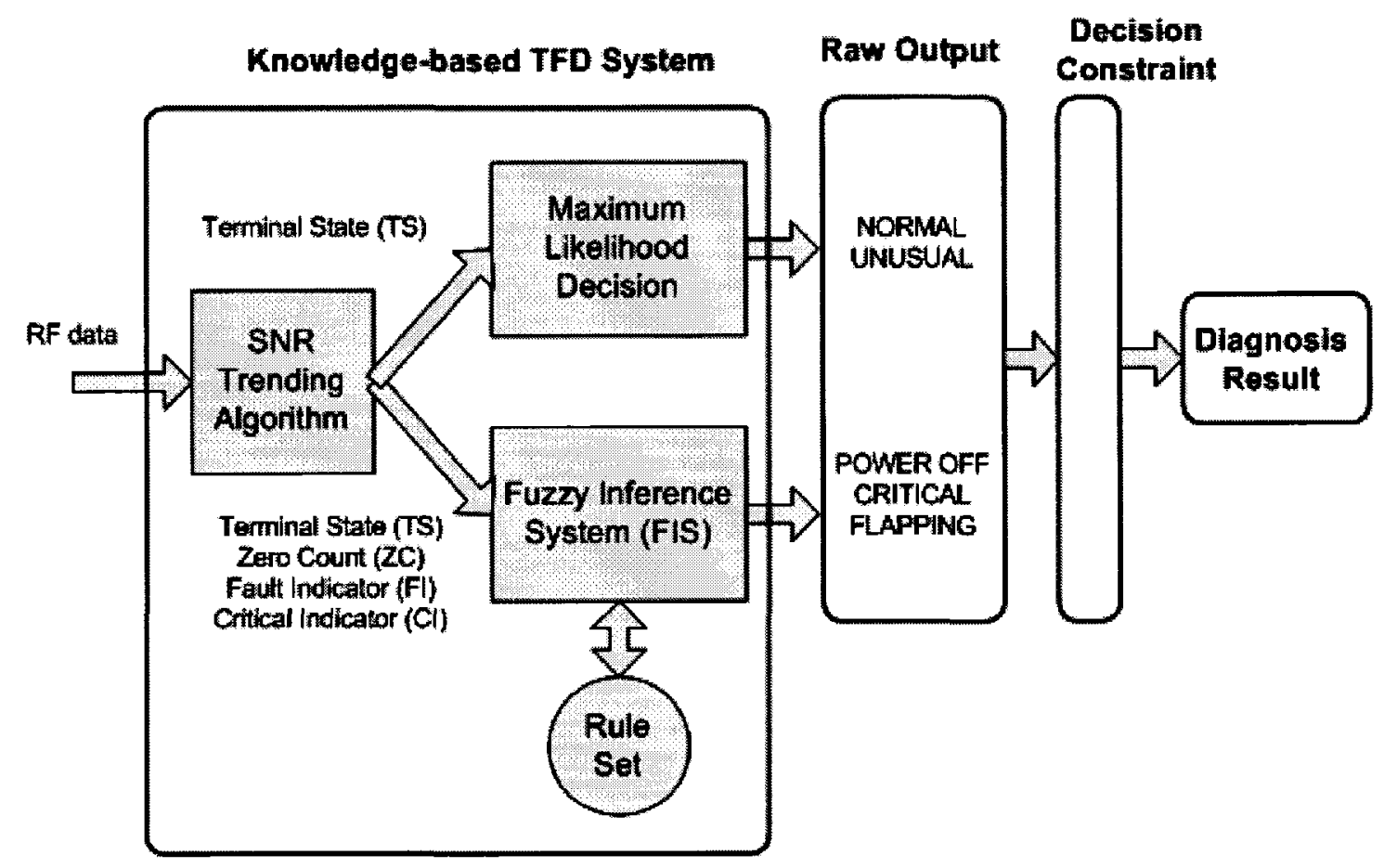

Figure 4.1: System architecture of knowledge-based TFD system 


\subsection{Mapping Forward Link SNR pattern to Events}

According to analysis and assistance from experienced satellite operators, the operating conditions of GT were categorized into five phenomena. The five phenomena are NORMAL, UNUSUAL, CRITICAL, POWER OFF, and FLAPPING. These phenomena are approved by Telesat, since they agreed that the five phenomena could cover most situations in daily operation. Each phenomenon corresponds to a certain event that influences the GT performance and produces different FLS signatures. Some impacts are self-recoverable, while others may cause permanent degradation to a satellite signal. In this section, the definitions of the five phenomena are given. In the following subsections, the signatures of each phenomenon and their corresponding causes are discussed in detail. All figures in this section are plotted using per-minute FLS data provided by Telesat.

\subsubsection{NORMAL Condition}

Each satellite GT has its own expected RF performance when it is deployed in the field. Satellite operator relies on FLS to monitor the operating condition of a GT. The expected FLS is normally 0.5 to $1.5 \mathrm{~dB}$ below the best FLS recorded during clear skies. The NORMAL condition implies that the FLS of GT stays above its expected level for most of the times over a two week period, in spite of the fact that the FLS might temporarily drop below the expected level.

When GT is operating under NORMAL condition, the FLS shows characteristic of smoothness with daily variations and sharp drops. The daily variation pattern of the FLS 
is caused by temperature changes in the surrounding environment and changes in atmospheric noise. Daily variations of FLS are inerratic. It reaches a daily peak at latenight and drops to the daily low at early morning or noon, depends on location and season. It can be well captured by plotting the weekly extent of FLS. Figure 4.2 shows FLS of Pond Inlet station from March 31, 2008 to April 7, 2008 where the FLS showed wave patterns.

Recent studies about how the weather impacts satellite signals showed that the rain attenuation is the most dominant impairment to the satellite signals [6]. FLS will experience sharp drops when the remote site is experiencing a heavy rain/snow-fall rate. Figure 4.3 shows the FLS from the Yellowknife station over a day, and it demonstrates the impact of the rain attenuation. The FLS of the Yellowknife station started to drop rapidly from 11:30 pm on July 24,2008 and returned to its normal level at 00:30 am on July 25,2008 . The largest gap between the bottom and its normal level was about $5 \mathrm{~dB}$. Among the impairment factors of the Ka-band signal, only the rain attenuation can damage the signal in such a short time. In extreme cases, the rain attenuation can degrade the Ka-band signal up to $20 \mathrm{~dB}$. Therefore, the impact of the rain attenuation may cause the GT to temperately $\log$ off the network, which results in no RF measurement.

Under the NORMAL condition, the GT may be affected by some unknown factors. The FLS pattern shown in Figure 4.2 shows such a phenomenon. At the end of week 13, the FLS of the Pond Inlet station started to drop, and it stayed low for about 3 days, and it finally went back to its expected level during the first day of week 14. If the FLS of GT can recover in a couple of days, then the system will presume that the GT is still 
operating under the NORMAL condition. However, if the FLS stays down or keeps dropping after a couple of days, then the system may report the GT as UNUSUAL. The upcoming section discusses the performance of GT under the UNUSUAL condition.

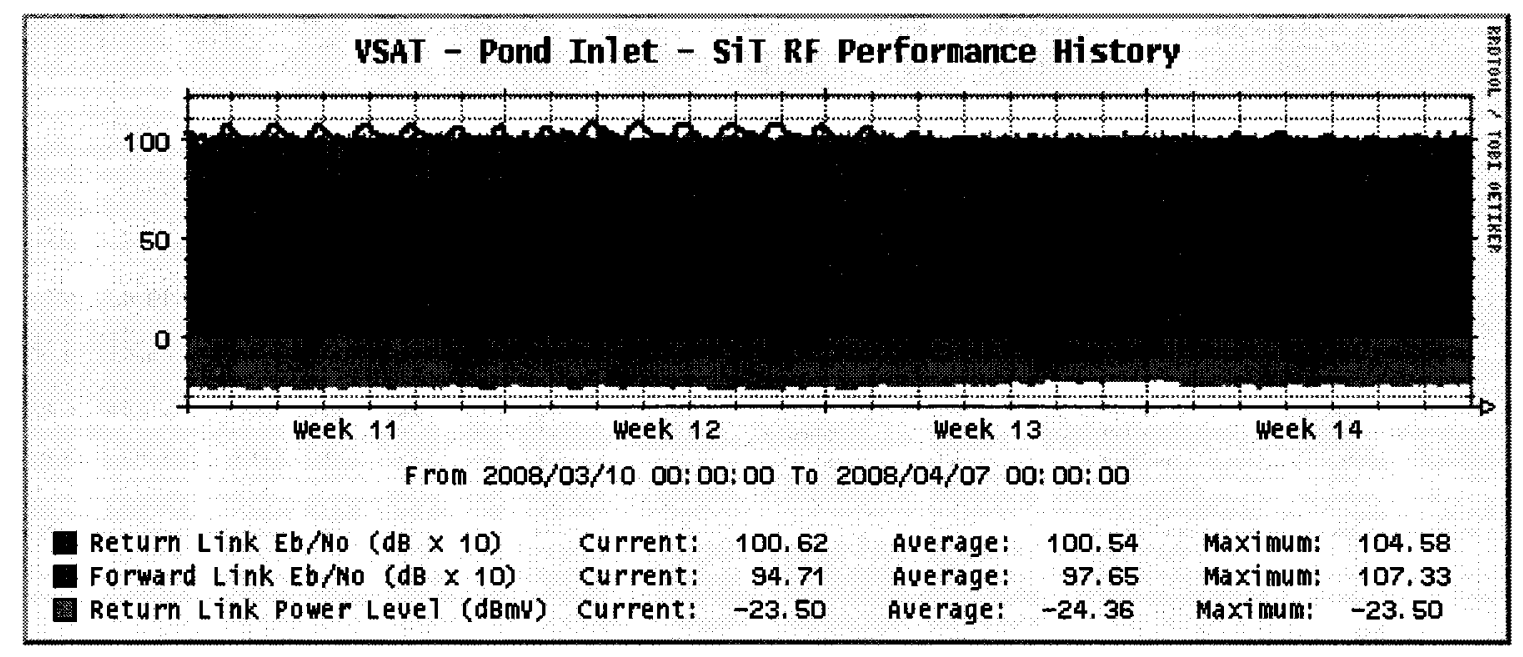

Figure 4.2: FLS with daily variation pattern under NORMAL condition

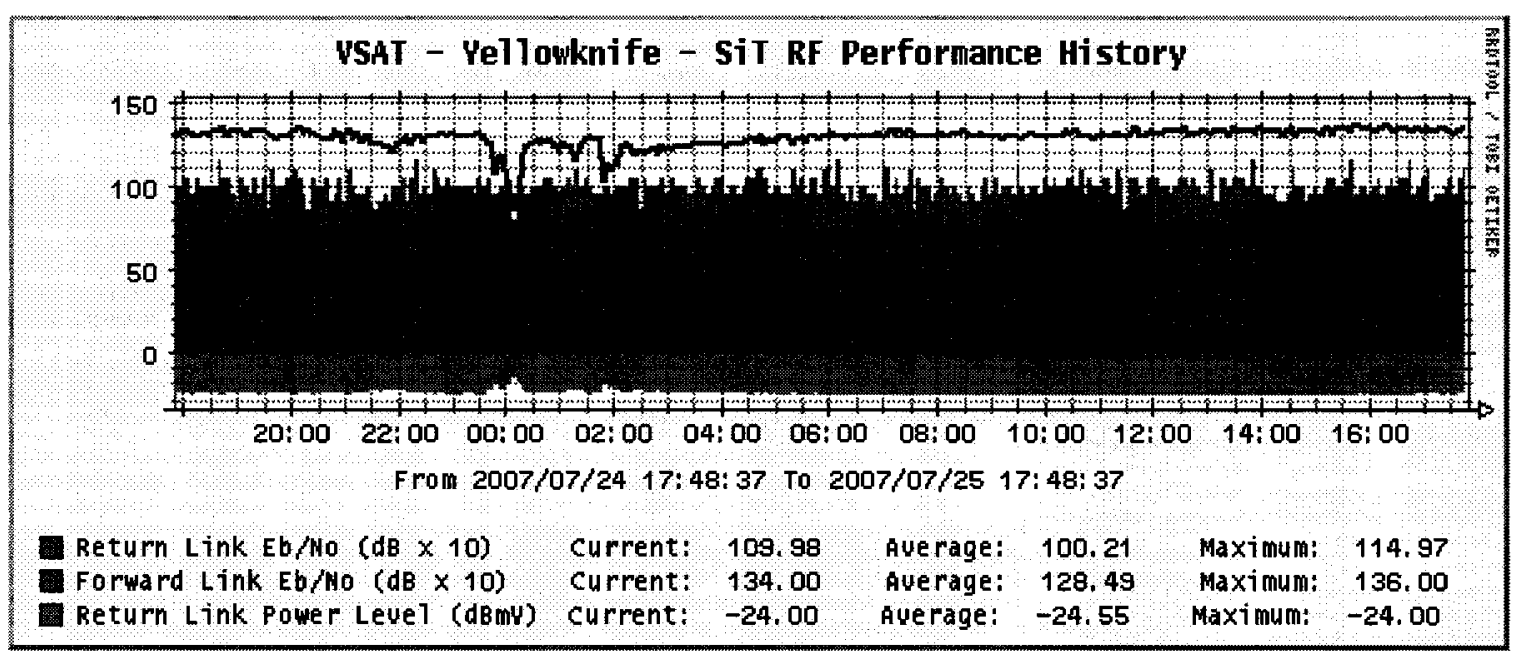

Figure 4.3: FLS with rain fade pattern under NORMAL condition 


\subsubsection{UNUSUAL Condition}

The UNUSUAL situation happens when the FLS of the GT stays below its expected performance level for most of the time during a week. The deteriorating performance of GT under the UNUSUAL situation is not reasonable, not acceptable, and should raise the concern of the satellite service provider.

The trends of FLS under the UNUSUAL situation follow two different patterns. The first type occurs when the FLS experiences a gradual drop over time and finally falls below the expected performance level. The causes of this type of condition are various and hard to predict. For example, a gradual shift of the satellite dish caused by loosing screws at the joint results in the UNUSUAL situation. When the location of the remote site enters the rain season, the FLS could also undergo extra signal attenuation. If the GT is near a lake or seashore, the increasing humidity of the surrounding environment could also results in the deteriorating performance of FLS. Figure 4.4 shows the gradual drop in the FLS over time at the Arviat station. Because this happened in the 29th week of the year where the location was in the raining season, the phenomenon should be likely the consequence of increasing humidity surrounding the areas. Wet antenna and snow build up on antenna can also result in a slow decrease in the FLS signal. An example of snow on the antenna is given in the next section.

The second type of UNUSUAL situation occurs when the FLS experiences a sharp drop and stays low for a long period of time. According to the satellite service provider, a suddenly shifted satellite dish could result in a sharp and permanent drop in the FLS. This type of fault is usually caused by strong winds or installation negligence. In Figure 4.5, 
the FLS from the Altin station suffered a step drop at the beginning of the $17^{\text {th }}$ week in 2006 and stayed down ever since. Moreover, the FLS took another step down at the end of week 24 , and it was finally terminated by the owner during week 29 . This case is a real case that happened in daily satellite operation. The satellite service provider sent a field technician to inspect the problem on site, and it was confirmed that the satellite dish displacement fault was caused by a loose screw at the joint. In this case, the step decrease of the FLS in this case was caused by a satellite dish displacement.

The FLS of the GT may experience a step increase under certain circumstances. The satellite manoeuvre may cause a step increase of the FLS. During daily operation, satellites may shift away from their expected position. Thus, most active satellites perform routine manoeuvres. During a manoeuvre, the LoS path between the GTs and the satellites may change, and this may result in a step decrease or an increase of the FLS. The step decrease belongs to the UNUSUAL situation category. The step increase of the FLS benefits the GT. As a result, the step increase will not be categorized into any of the five phenomena defined in this thesis. Figure 4.6 shows the effect of satellite manoeuvre on the FLS. 


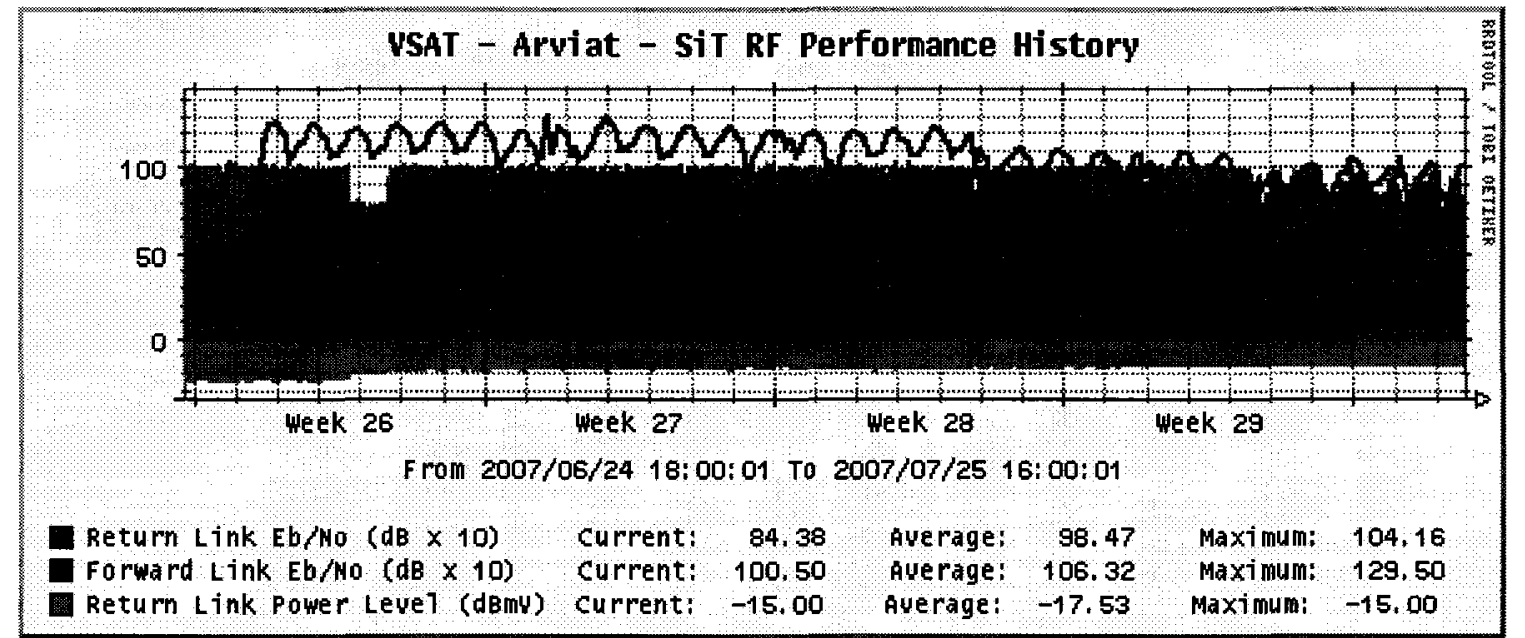

Figure 4.4: Deteriorating performance of Arviat station under UNUSUAL condition

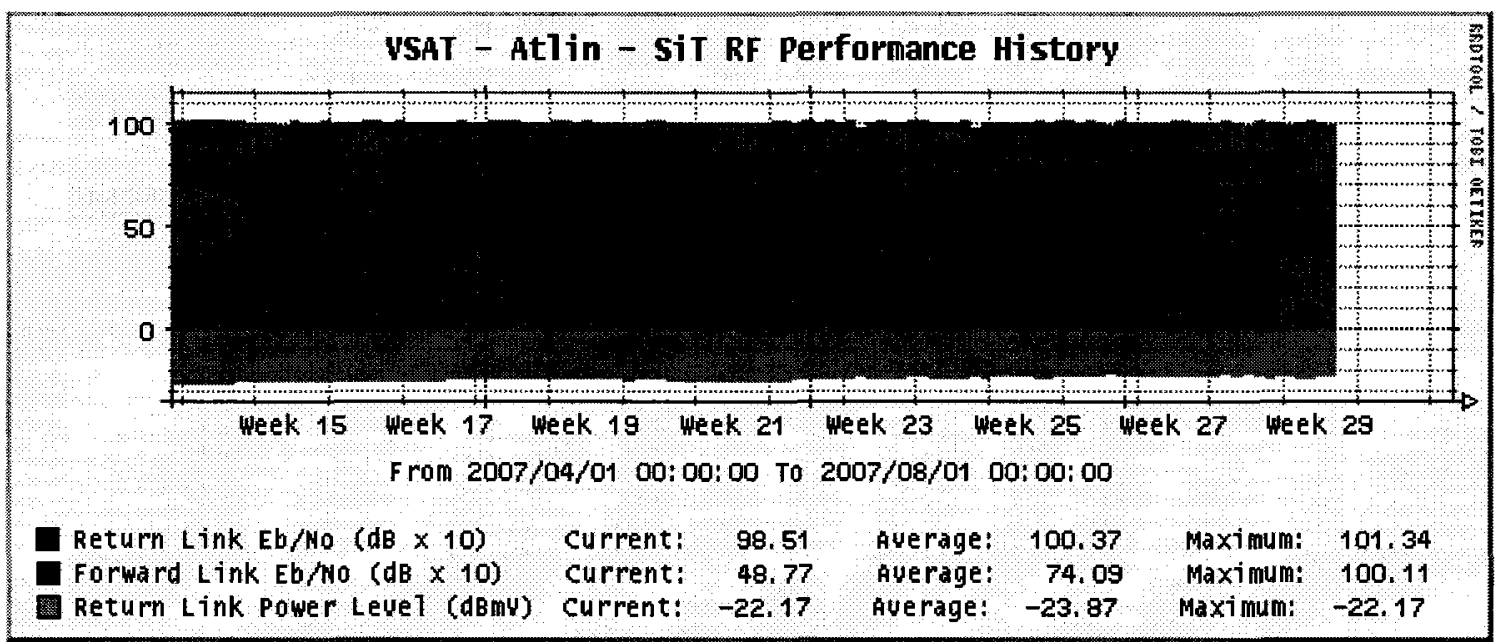

Figure 4.5: Deteriorating performance of Altin station caused by antenna displacement

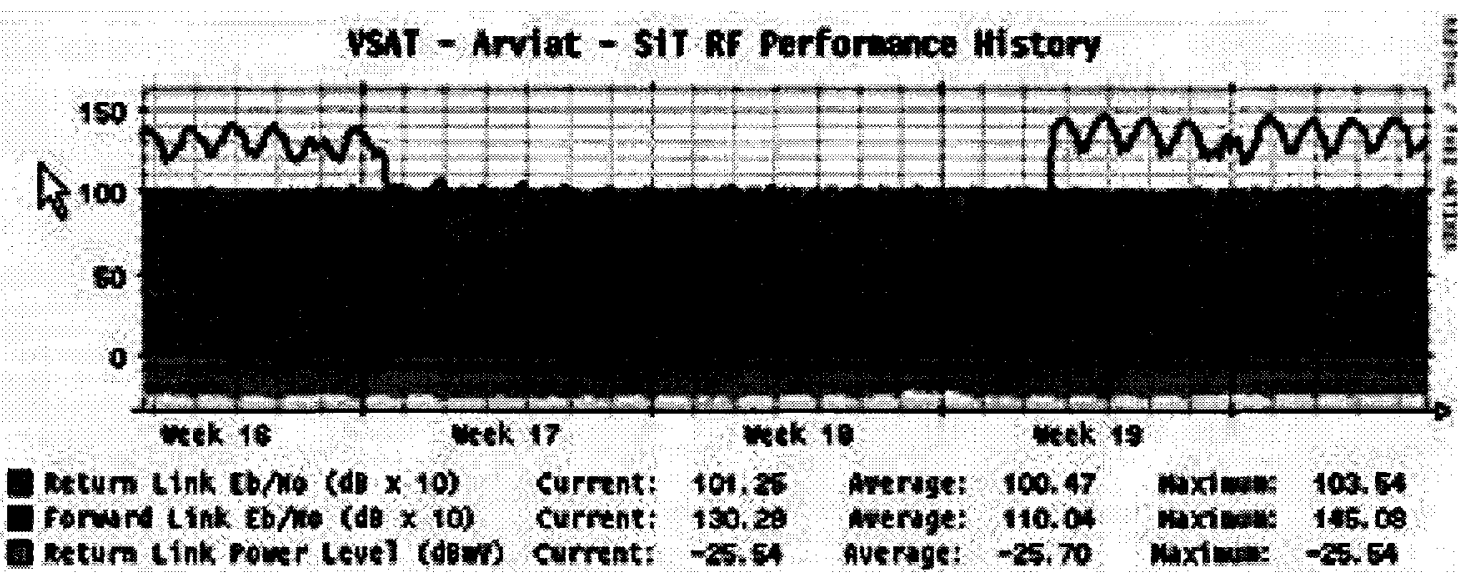

Figure 4.6 FLS performance under satellite manouvre 


\subsubsection{CRITICAL Condition}

A GT is said to be in the CRITICAL condition when its FLS is continuously below the expected performance level for a long period of time (usually two weeks). The CRITICAL situation is an extension of the UNUSUAL situation. Its cause is similar to that of the UNUSUAL situation. The UNUSUAL situation represents the influence of the disturbing effect at its early stage. The CRITICAL situation indicates that the influence of the disturbing effect extends beyond the expected duration. In Figure 4.7, the FLS from the Pond Inlet station illustrated typical CRITICAL behaviour when the FLS dropped slowly in the first two weeks and stayed low for another three weeks before the problem was resolved. This is another real world example provided by the satellite service provider. The satellite service provider confirmed that the event was caused by snow build up on the satellite dish. The Pond Inlet is in the north part of Nunavut, and it is the largest hamlet above the $72^{\text {nd }}$ parallel. In the winter of 2007 , all of Canada experienced a record breaking snowfall and so did the Pond Inlet. Fortunately, the problem was solved by a simply clean up of the snow on the satellite dish. On the third day of week 3 in 2008 , the Pond Inlet station went back online and operated normally. In conclusion, when the GT is under the CRITICAL condition, the service provider should take action immediately in order to solve the problem. 


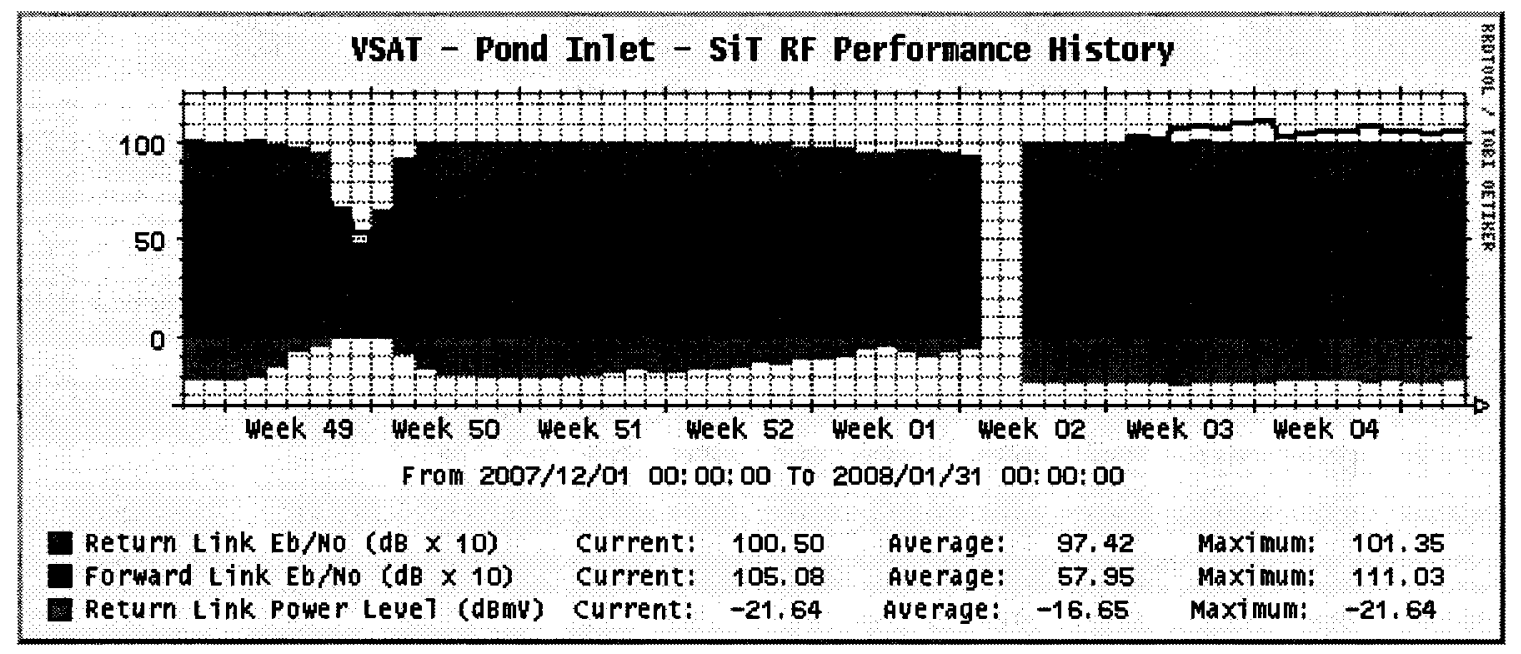

Figure 4.7: Deteriorating performance of Pond Inlet station under CRITICAL condition

\subsubsection{POWER OFF Condition}

If a GT does not have RF data responses for several hours, it is considered POWER OFF. The POWER OFF phenomenon is easily observed. If the incoming RF data from a GT contains consecutive zeros for hours, the GT can be identified as POWER OFF.

Several incidents can result in GT power off. User triggered power off is one of the most frequent scenarios. In Figure 4.8, the Terrace station logged off the satellite network from 22:30 at night until 6:00 am the next morning, and there is no RF data detected at GT until it logged on again. Temporary failure of pulling RF data can also result in a FLS performance similar to a power cut-off. Extreme weather conditions on site may also drive the ground terminal to a temporarily no-response state. In Figure 4.9, the FLS of the Iqaluit station dropped rapidly at $22: 00$ on July $25^{\text {th }}$ and did not have RF data for two short periods between $03: 00$ am and $06: 00$ am on July $26^{\text {th }}$. This was due to temporary failure of pulling RF data at the GT. Therefore, when there is no RF data available for long periods of time, the GT will be considered as POWER OFF. 


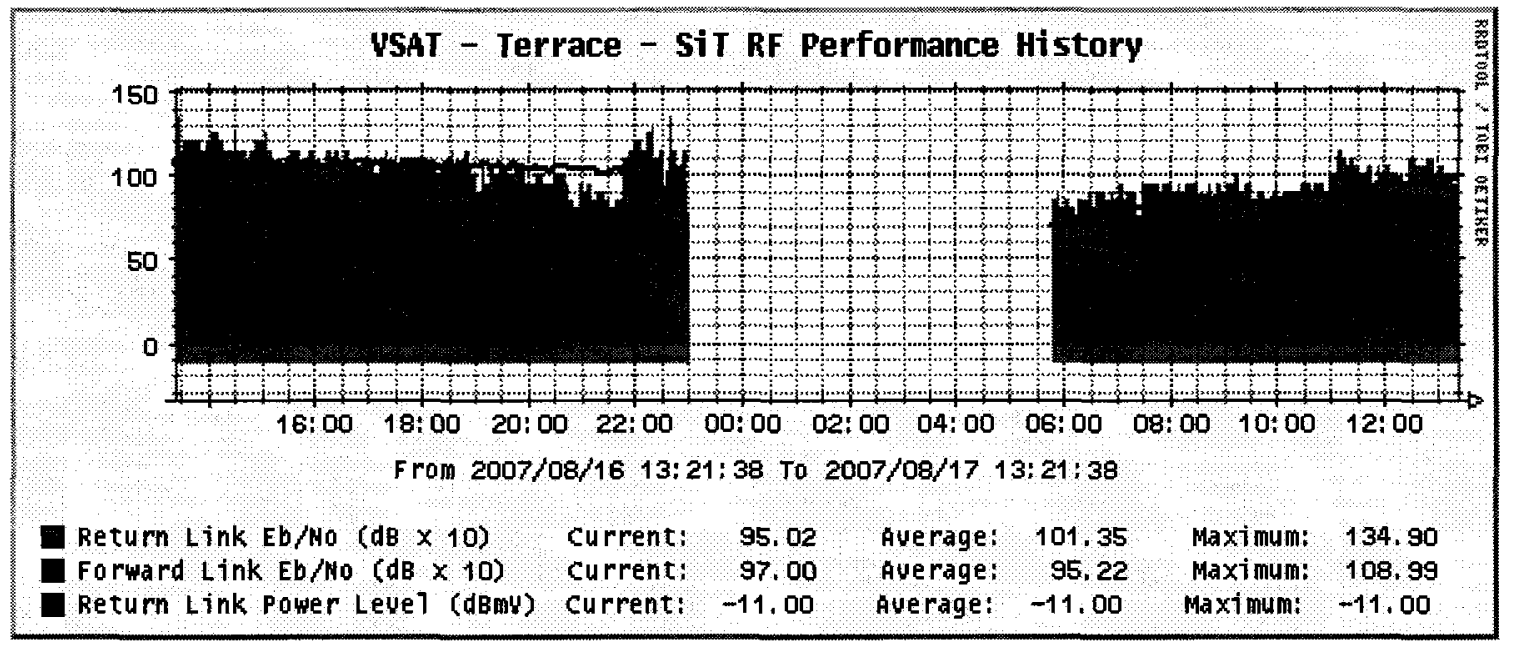

Figure 4.8: FLS pattern for POWER OFF

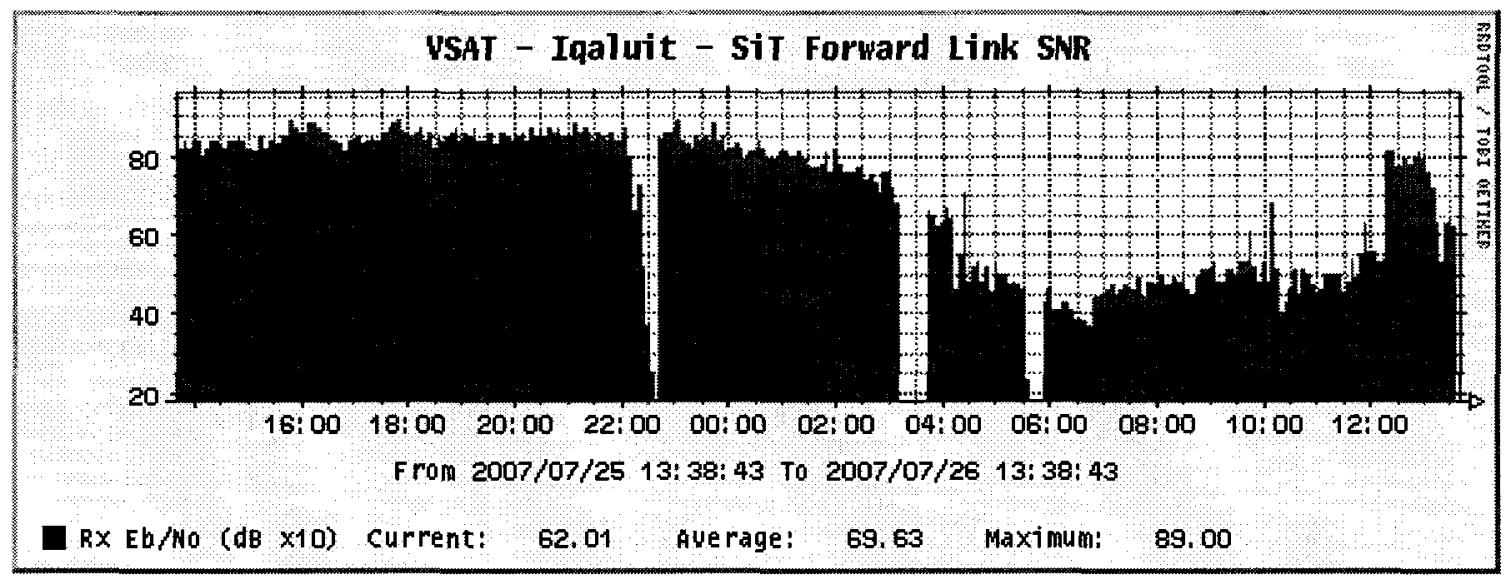

Figure 4.9: Temporary failure of pulling RF data due to extreme weather condition

\subsubsection{FLAPPING Condition}

FLAPPING is one of the most annoying faults in the satellite network operation. The FLAPPING situation happens when the GT hardware is experiencing a hardware malfunction. When a hardware malfunction occurs, the GT may log on and off the satellite network frequently in short intervals. Figure 4.10 illustrates the behaviour of a flapping modem. The modem's return link unit started to fall on Monday March 31, 2008 with some fitful readings on the return link SNR. It was not long before the exceptional 
behaviour spread to the entire functionality of the modem. The next day, the RF measurements of both the FLS and the RLS experienced an intermittent record. In addition, the user on site reported unstable network conditions to Telesat. By incorporating all the information available, the GT was diagnosed as FLAPPING. If the GT is FLAPPING, the satellite service provider has to send new equipment to replace the faulty hardware.

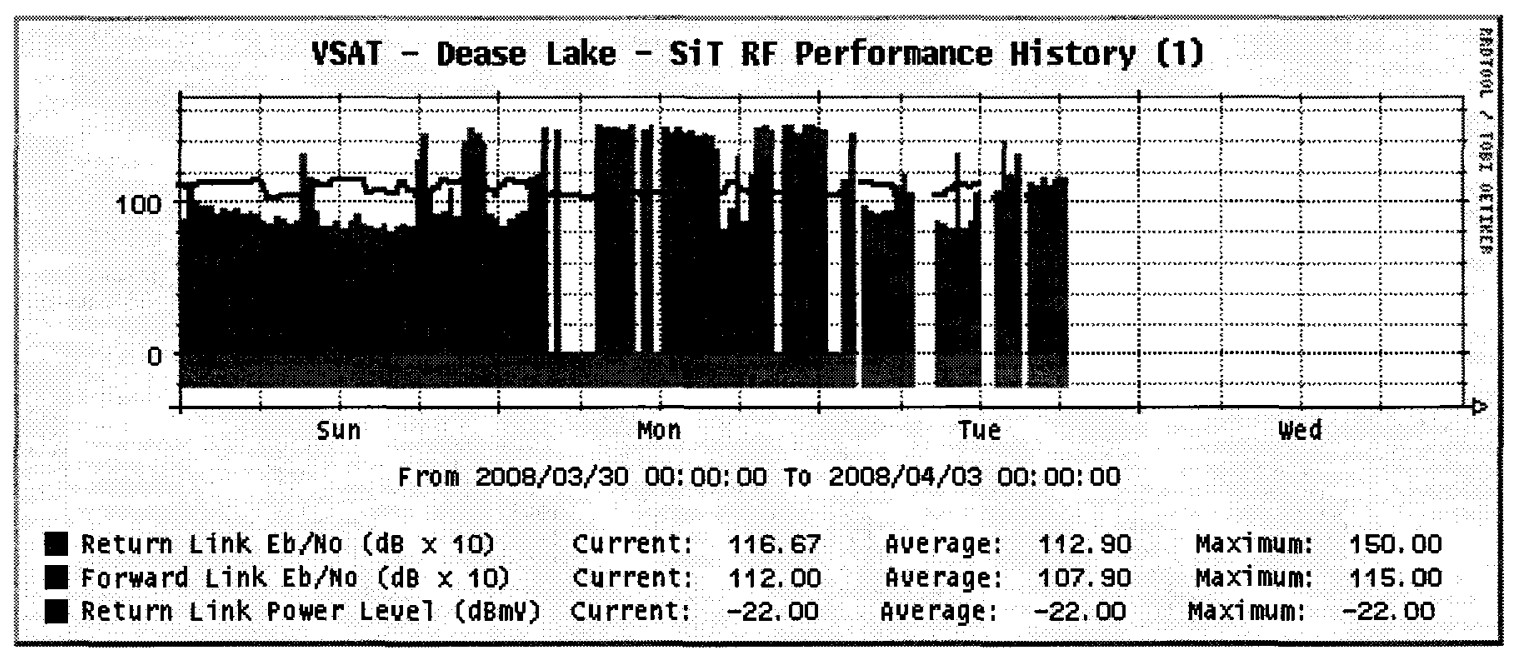

Figure 4.10: FLS pattern for flapping modem

These five phenomena are categorized from a large number of archived RF data with the help of experienced satellite operators. As Telesat confirmed, they cover most of the scenarios in daily satellite operation. As a result, the output of the knowledge-based TFD system expresses the status of the GT in terms of the five phenomena. 


\subsection{SNR Trending Algorithm}

The last section demonstrated the FLS patterns for each phenomenon in the previous section. In the knowledge-based TFD system, the trending patterns of SNR are very important when making diagnosis decisions. In the broadband satellite system, FLS is measured every minute. However, if the system only looks at a single FLS recorded, it will not be able to see the trend of FLS. In order to refine the trend of FLS, instead of monitoring FLS every minute, a bulk of measured FLS needs to be processed together. The SNR trending algorithm generates four indicators in order to represent the impending trends and current conditions of the GT. The following section explains the SNR trending algorithm and compares the two operation modes of the algorithm.

\subsubsection{SNR Trending Algorithm}

In the broadband satellite system, FLS measurements are available every minute. If the system looks at the FLS samples individually, it can only tell us the current level of FLS. On the other hand, the trending patterns of the FLS can be easily identified when looking at a series of FLS samples. Thus, keeping track of previous FLS samples helps us identify the trends of the FLS. The SNR trending algorithm developed for the TFD system used a sliding window method in order to determine the FLS trending patterns.

A Sampling Period (SP) is a fixed period where a number of FLS samples can be collected and processed together. The SP determines the time interval of each outcome inferred from the TFD system. In other words, the TFD system makes a decision for every SP. The SP is chosen to be hourly based, and the average FLS is calculated for 
each SP. Furthermore, calculating the average FLS during SP eliminates the fluctuation of the signals caused by measurement errors and instant changes in the weather conditions. The average FLS provides us with an average performance level of GT during each SP. In the SNR trending algorithm, a Sliding Window (SW) consists of multiple SPs. The relationship between SPs and SW is illustrated in Figure 4.11. A diagnosis decision will be made based on the information from all the SPs within the SW. As time progresses, the $\mathrm{SW}$ moves forward, and the information from the most recent $\mathrm{SP}$ replaces the information from oldest SP. The size of the SP and SW influences the output of the SNR trending algorithm. In this experiment, it was decided that the length of SPs would be hourly based, and the size of SW would be weekly based according to heuristic. The way that $\mathrm{SP}$ and $\mathrm{SW}$ are chosen is explained in the next section.

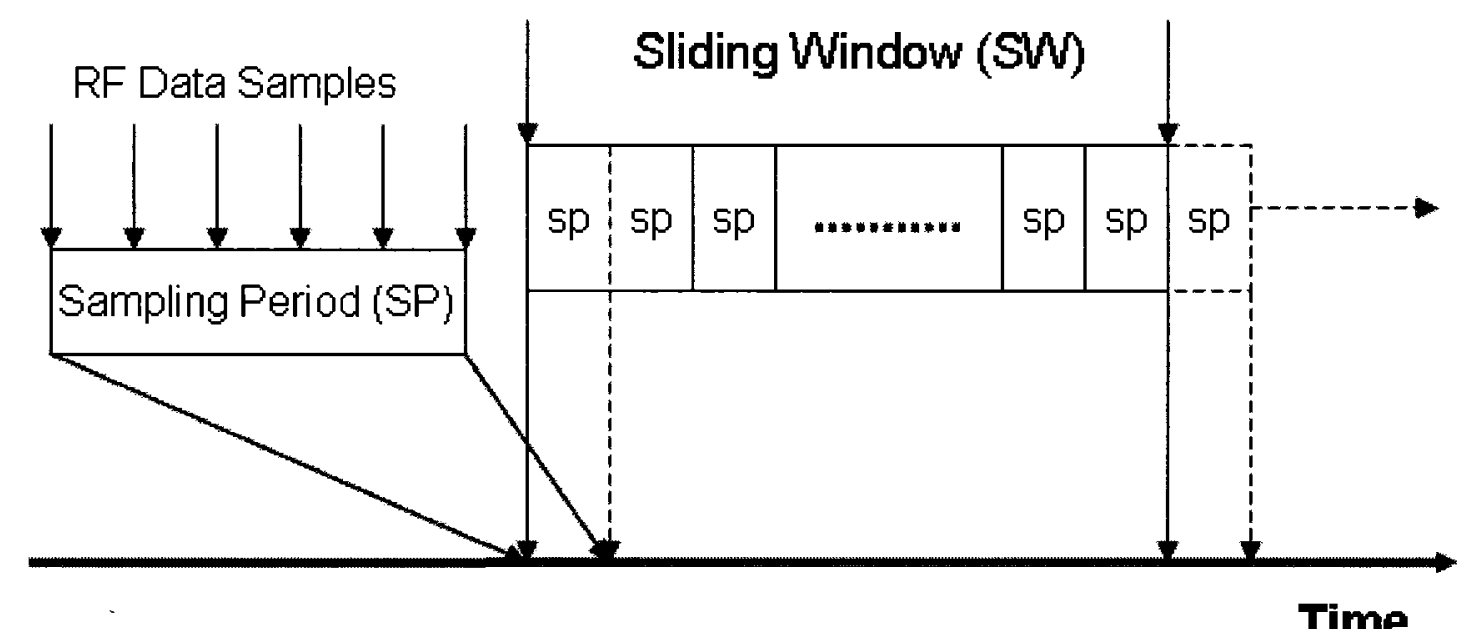

Figure 4.11: Relationship between Sampling Period and Sliding Window 
The trend of FLS is summarized in the Terminal State (TS), computed numerically in the algorithm, described as below:

Initialization Phase: Expected FLS, SP, SW

\section{Algorithmic Phase:}

1. Calculate the average FLS for the most recent SP.

2. Compare the average FLS with the expected FLS of the given GT and determine the Status according to the following rules:

If average FLS $\leq$ expected FLS then Status = 1;

If average FLS $>$ expected FLS and TS $>0$ then Status $=-1$;

If average FLS $>$ expected FLS and TS $=0$ then Status $=0$;

3. Calculate the TS using the Status of each SP within the SW according to:

$$
T S=\sum_{i=0}^{S W \text { Size }} \text { Status }_{i}
$$

4. Move forward to the next SP and go to step 1.

TS has a minimum value of 0 and goes up to the number of SPs in SW. Its value shows the net number of SPs where the GT is operating below the expected threshold during the SW period. Therefore, TS indicates the performance trend of GT over the SW period. TS is also the core element in deciding whether the GT is under the NORMAL or UNUSUAL condition. If the value of TS is zero, this means that the satellite is operating above its expected performance level most of the time, and the GT is operating under the NORMAL condition. If the value of TS is low, this means that the satellite is operating in good condition most of the time during the SW period, and the GT should be considered 
as operating under the NORMAL condition. When the FLS of the GT is operating below the expected performance for most of the times in the SW, the TS will have a large number that is close to its maximum value. In this case, the GT should be reported under the UNUSUAL condition. If TS stays at its maximum value, this indicates that the GT has underperformed for a period that is beyond what was expected. The GT is under the CRITICAL condition. The decision threshold of TS is crucial in the fault diagnosis system. If the threshold is too low, then the system may report the UNUSUAL situation too early. The drawback of having a high threshold is the opposite. The Maximum Likelihood method was proposed in order to diagnose the NORMMAL and UNUSUAL conditions. The method is discussed in Section 4.5 in detail.

\subsubsection{Output of SNR Trending Algorithm}

TS only shows the time that a GT operates under the expected performance during the last SW. This information is not enough to represent the thinking patterns of an experienced satellite operator. As a result, the SNR trending algorithm generates three other parameters to fully represent the expert thinking pattern. The three parameters are Zero Count (ZC), Fault Indicator (FI), and Critical Indicator (CI). The following section describes the meaning of these parameters and discusses how they are generated.

$\mathrm{ZC}$ counts the number of null data samples collected during the SP. It is the key indicator when determining the POWER OFF condition. If the value of $Z C$ is close to the number of RF data points in a SP, then the current SP is considered a highly suspected POWER OFF period. ZC also plays a support role in the decision of the CRITICAL and FLAPPING conditions. When $\mathrm{SP}=3$ hours, the maximum value of $\mathrm{ZC}$ is 180 . 
FI describes the number of unstable SPs during the last 24 hours. The definition of unstable SP in the SNR trending algorithm can be written as:

IF ( $\mathrm{ZC}>20 \% * \mathrm{SP})$ AND (ZC not equal to SP) THEN SP is unstable According to this statement, a SP is said to be unstable if the number of null RF data during the period exceeds $20 \%$ of the total RF data point in the SP. There are several drawbacks and advantages in using this method to describe the flapping condition. The ZC may misrepresent the FLAPPING conditions when the current SP is actually in the POWER OFF condition. For example, if the POWER OFF period shown in Figure 4.8 is divided by two SPs, then each SP in the SNR trending algorithm will report the current SP flapping instead of POWER OFF. FI counts the number of unstable SPs over the last 24 hours and decides whether the GT is flapping using FIS. The flapping conditions caused by a single misrepresented unstable SP will be discarded by the FIS. In additional, using FI can eliminate some fake FLAPPING conditions caused by temporary failure of pulling RF data at GT. Although temporary failure of pulling RF data results in a similar FLS pattern as the FLAPPING condition, they are different in terms of continuity. Temporary failure of pulling RF data may only happen once a while and will recover in a short time. The FLAPPING condition in the GT is usually caused by hardware malfunction. Therefore, when real flapping conditions occur, it is likely that the GT will behave flapping all day long.

$\mathrm{CI}$ counts the number of SPs where TS is maintaining at its maximum value over the last two days. When TS is at its maximum value, it indicates that the GT underperforms during all SPs in a SW. The system can not tell whether the situation is getting better or 
worse. CI describes the trend of TS after it has reached its maximum value. For each SP during the last two days, if the TS of the current SP reaches its maximum value, the CI increases by 1 . When the value of $\mathrm{CI}$ is low, this means that the GT has underperformed for weeks, but the situation is not getting worse. A high value of CI indicates continued deteriorating performance of the GT.

\subsubsection{Parameter Sensitivity Analysis}

The selection of SP and SW influences the outputs of the SNR trending algorithm. It also affects the design of the FIS. This section describes the empirical approach of selecting suitable SP and SW for diagnosing the operating conditions of satellite GTs.

FLS samples of the Hazelton station from February 1, 2007 to July 31, 2007 are selected in order to demonstrate the importance of choosing reasonable SPs and SWs. The FLS of the Hazelton station from February $1^{\text {st }}$ to July $31^{\text {st }}$ is plotted in Figure 4.12 . Each value in the figure is a three hour average of real-time FLS. As shown in Figure 4.12, the 3 hour average FLS undulated around $8 \mathrm{~dB}$ from February $1^{\text {st }}$ to the end of May. Then, the FLS started to drop gradually from the end of May and stayed low for about three weeks. FLS restored to its expected value and continued to undulate around $8 \mathrm{~dB}$ at the end of June. This phenomenon is a typical UNUSUAL event. The UNUSUAL condition of GT is captured by monitoring the change of TS. However, using different SP and SW result in different TS patterns using the same data set. The TS of the Hazelton station from February $1^{\text {st }}$ to July $31^{\text {st }}$ with different SPs and SWs are plotted in Figure 4.13, Figure 4.14, and Figure 4.15. A detailed analysis is given below. 


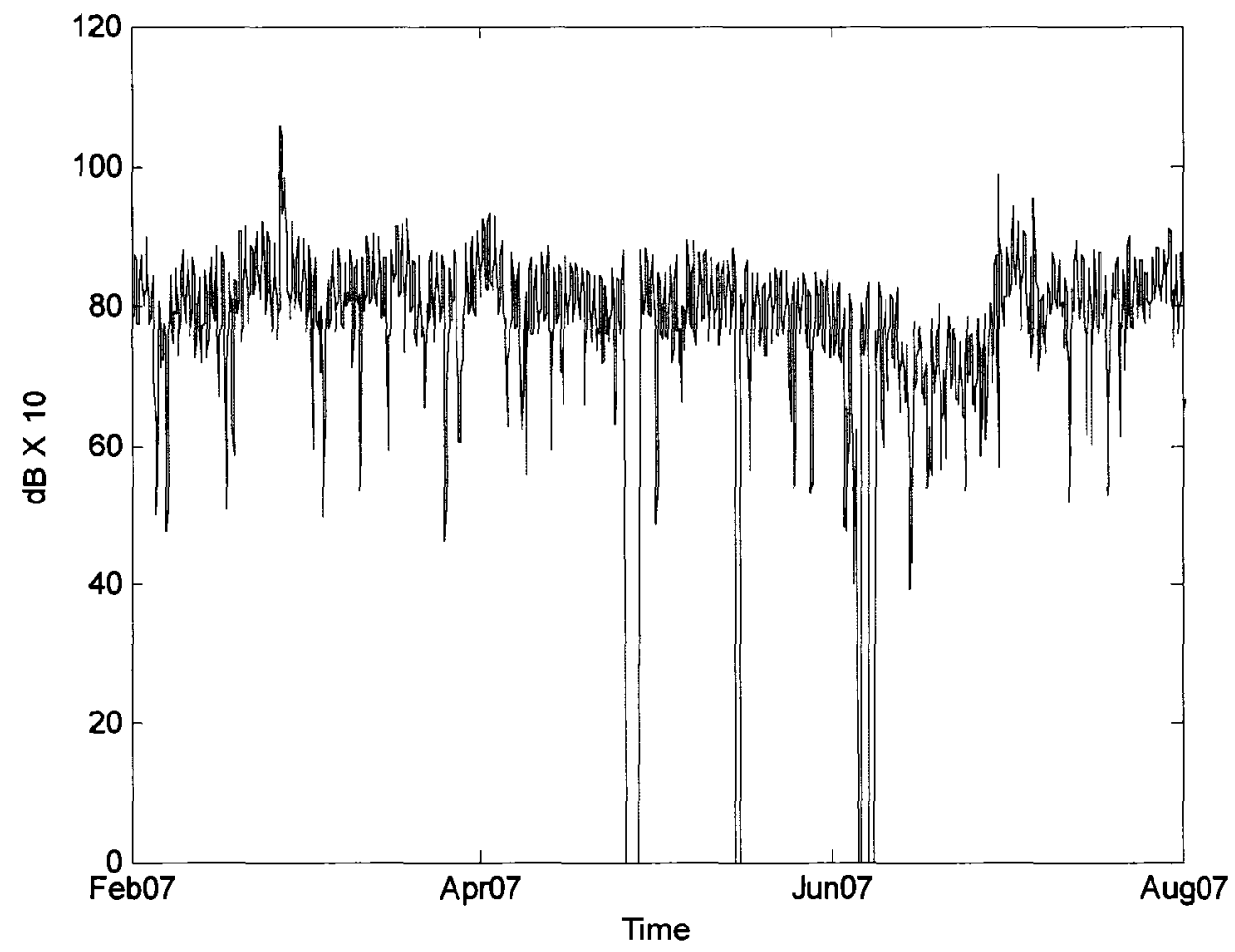

Figure 4.12: FLS of Hazelton station (3 hour average)

First of all, we fixed SP and let SW float. The size of SW decides the number of historical SPs involved in the decision of the TS for the current SP. It also determines the maximum value of TS. In the design of the TFD system, the size of SW is crucial to the accuracy of the diagnosis of the UNUSUSAL condition. For a given SP, if SW is small, then the number of the historical SPs used to decide the current TS is small. Therefore, TS can only represent the change of FLS in a short time period. When generating TS with FLS trends extracted from a short time period, the trend of TS may not be able to present the trend of FLS accurately. TS may stay at a value close to its maximum value, when a small SW is used. If SW is large, then TS will be generated base on more historical data. However, if SW is too large, the decision of TS may involve too many SPs. Using a large 
SW may delay the decision made on UNUSUAL conditions. Figure 4.13 shows the measurement of TS with different SWs, when fixing SP at 3 hours. In sub-plot (1) of Figure 4.13, SW was set to one day where TS has maximum value of 8 . TS was decided based on the RF data collected on the previous day. Thus, TS represented the trend of FLS in the prior 24 hours only. Because SW was very short, TS changed rapidly. TS upheld at its maximum value during the UNUSUAL period, which occurred from the end of May to the end of June. The trend of TS did not clearly identify the UNUSUAL condition. The pattern of the UNUSUAL condition started to show up as the size of SW increased. From sub-plot (2) to (6) in Figure 4.13, the UNUSUAL condition of the Hazelton station appears as a peak with a different maximum value. The maximum value of TS for the UNUSUAL condition increased when the SW increased. According to our experience, using an SW equal to 14 days can clearly indicate the UNUSUAL event.

Next, we fixed SW at 14 days and allowed SP to change. SP is the sensitivity of the TFD system. If SP is too short, TFD system will become reactive. If SP is too long, the TFD system will become inactive. For this reason, choosing a proper SP is crucial. Figure 4.14 illustrates the trends of TS under different SPs. The trends of TS under different SPs are similar to each other, but with different maximum values. Although the trends of TS can not be separated by shape, different SPs affect the decision sensitivity of the TFD system. For diagnosing the operating condition of GTs, the SP was chosen to be 3 hours for two reasons. First of all, according to past experience, the duration of weather-related short-term impacts on the GT performance is usually less than 3 hours. Therefore, using a 3 hour average of FLS can represent the average performance of GT during SP. Secondly, 
choosing an SP equal to 3 hours provides a suitable decision interval for the satellite service provider.

Finally, we fixed the maximum value of the TS at 112 and then adjusted the size of the SP and the SW so that the maximum of the TS can reach 112. Figure 4.15 shows the FLS plotting with different SPs and SWs. In subplots (1) and (2), the trend of the TS showed rapid changes in value and stayed at its maximum for a period of consecutive SPs. This means that the chosen SW is too small to fully represent the operation condition of the Hazelton station. On the other hand, trends of the TS in subplots (5) and (6) from Figure 4.15 changed slowly and did not get close to their maximum value. Subplots (5) and (6) from Figure 4.15 showed the proper trends of TS for the Hazelton station. As a result, choosing $\mathrm{SP}=3$ hours, $\mathrm{SW}=14$ days, and $\mathrm{SP}=4$ hours, $\mathrm{SW}=20$ days satisfies our design.

Although different SP and SW result in a similar TS pattern, choosing a suitable SP and SW provides accuracy trends for FLS and gives proper decision sensitivity. Based on an empirical analysis, $\mathrm{SP}=3$ hours and $\mathrm{SW}=14$ days was proposed for the knowledgebased TFD system. 
(1) $S W=1$ days $\operatorname{MAX}(T S)=8$

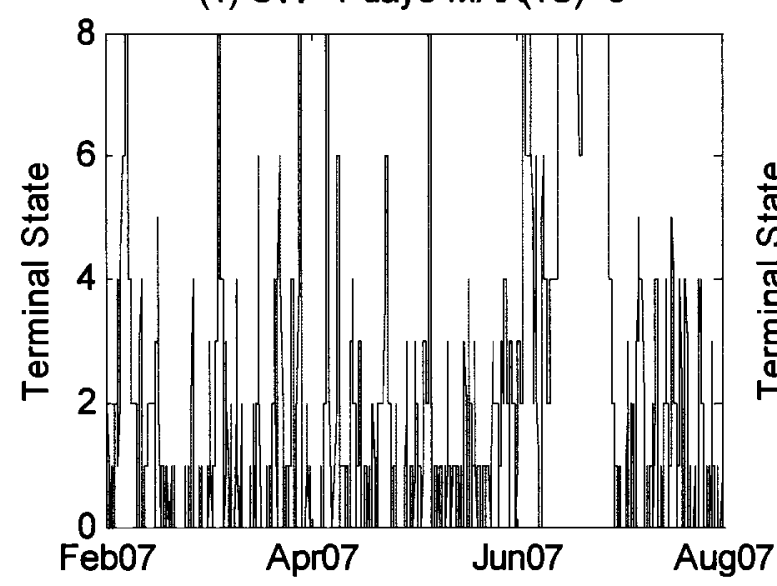

(3) $S W=7$ days $\operatorname{MAX}(T S)=56$

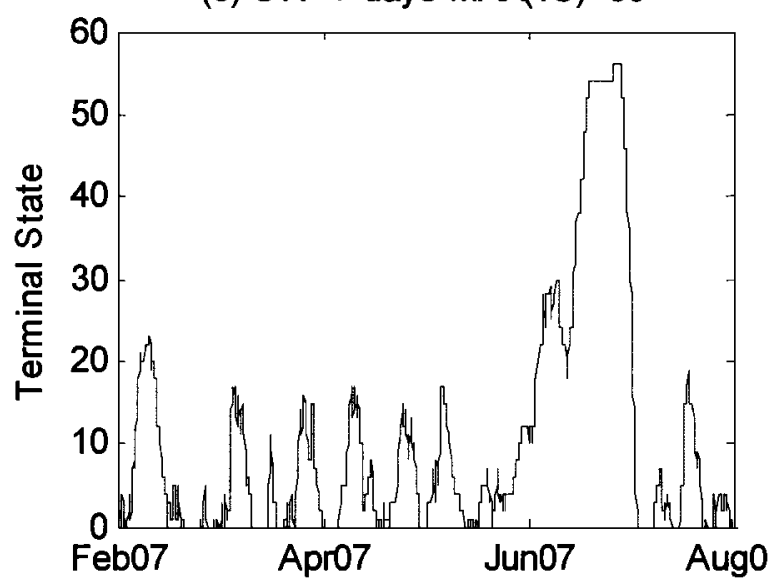

(5) $S W=14$ days $\operatorname{MAX}(T S)=112$

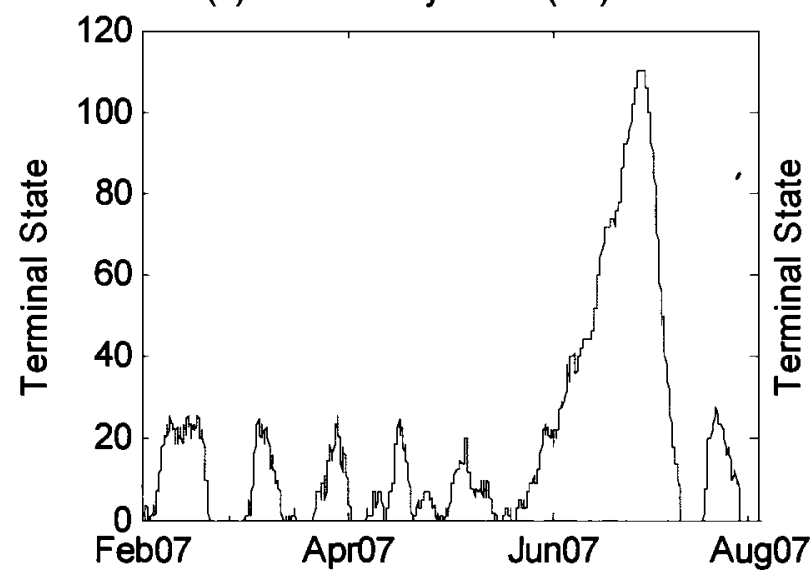

(2) $S W=3$ days $\operatorname{MAX}(T S)=24$

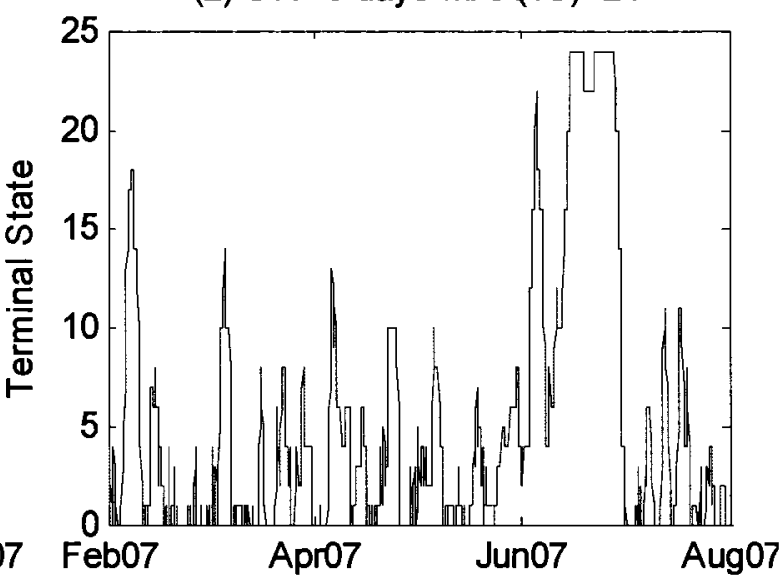

(4) $S W=10$ days $\operatorname{MAX}(T S)=80$

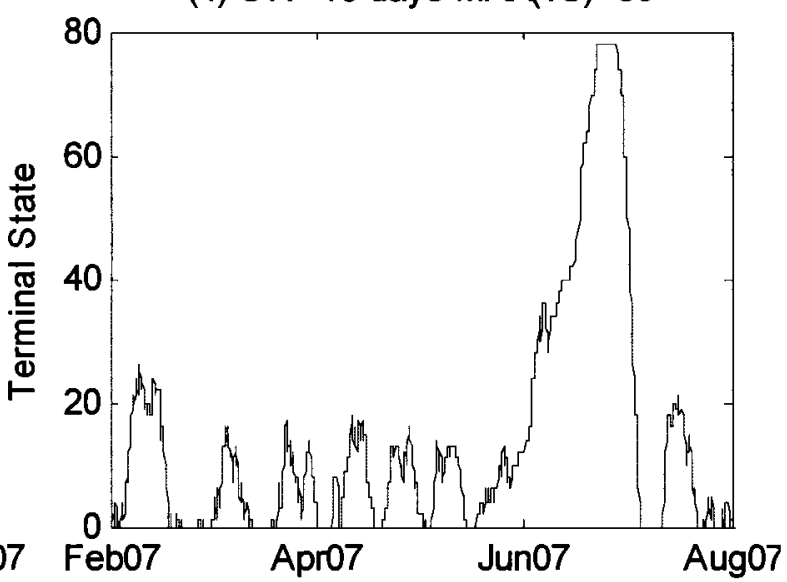

(6) $S W=21$ days $M A X(T S)=168$

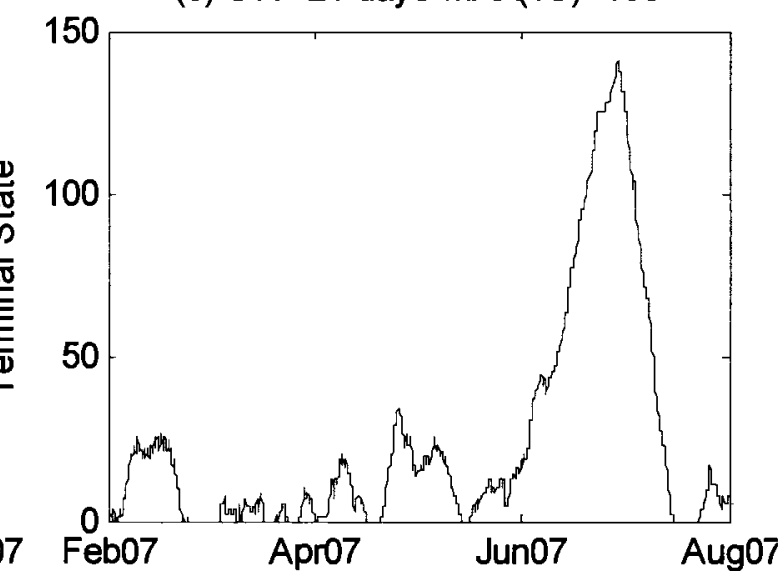

Figure 4.13: Measurement of Terminal State under different $S W$ with $S P=3$ hours 

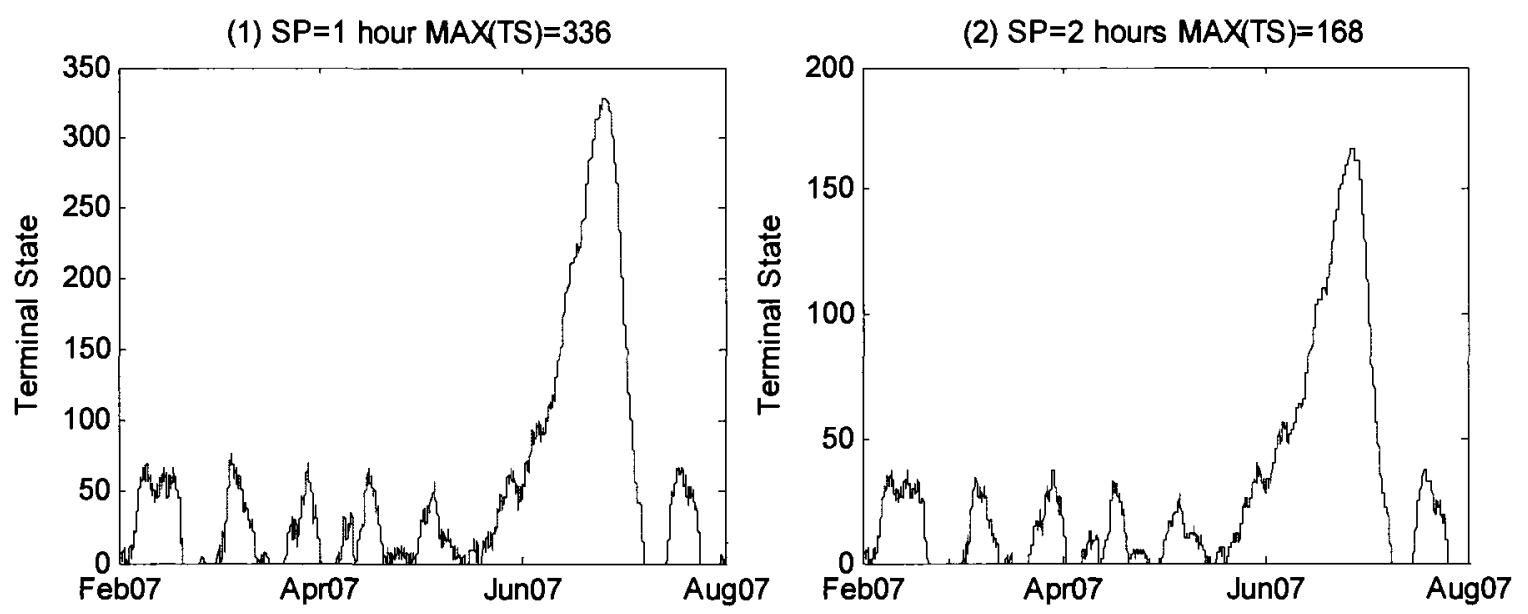

(3) $S P=3$ hours $\operatorname{MAX}(T S)=112$

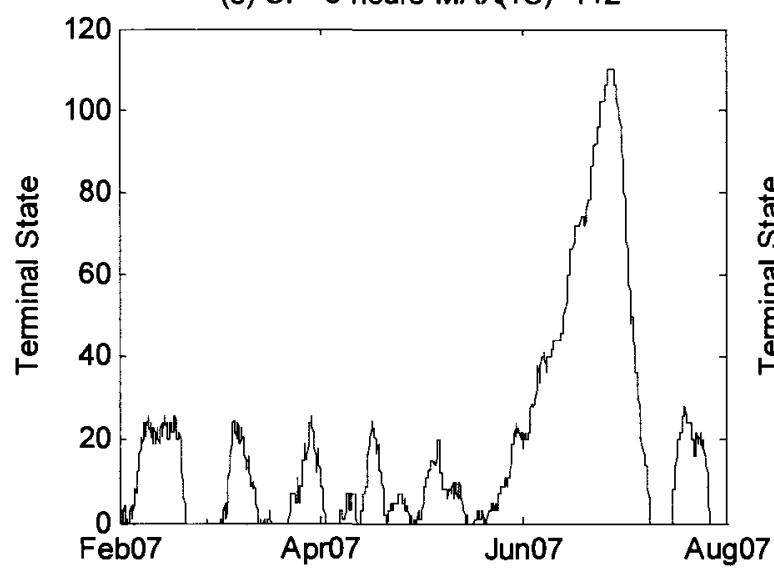

(4) $\mathrm{SP}=4$ hours $\operatorname{MAX}(T S)=84$

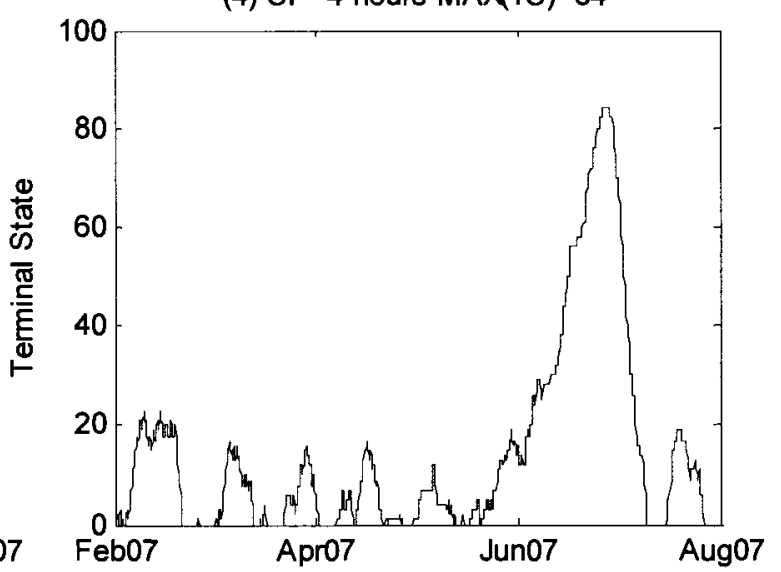

(5) $\mathrm{SP}=3$ hours $\operatorname{MAX}(T S)=42$
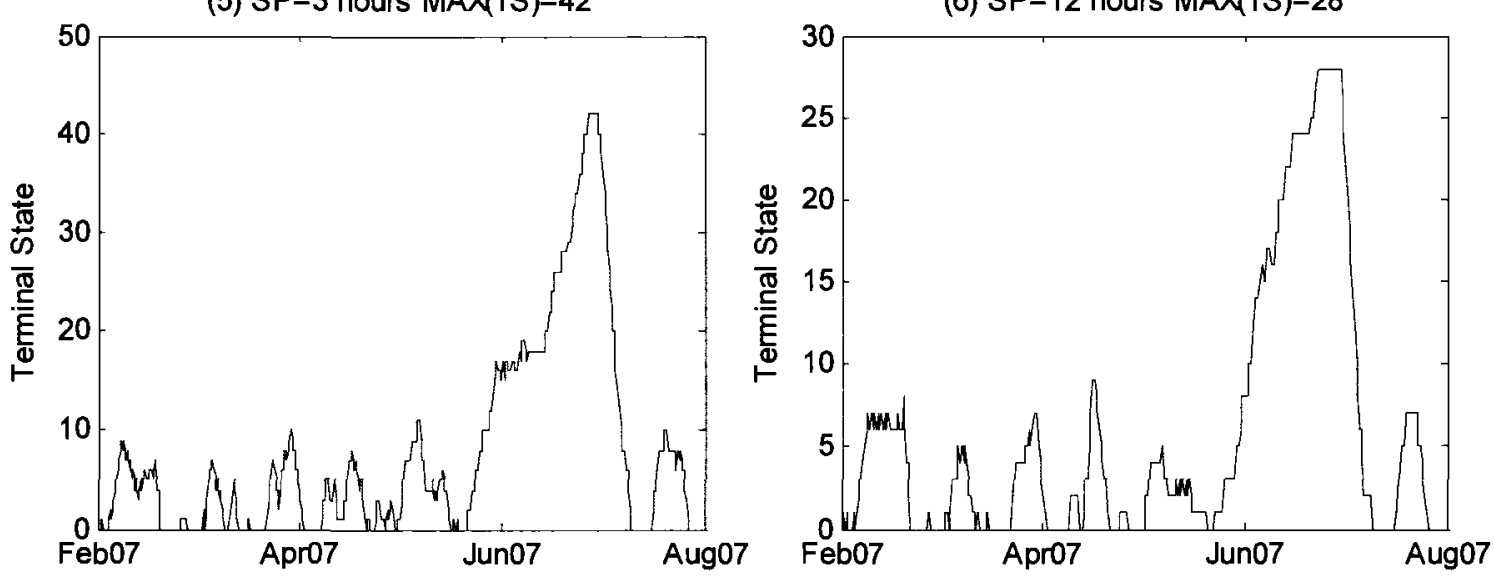

Figure 4.14: Measurement of Terminal State under different SP with SW = 14 days 

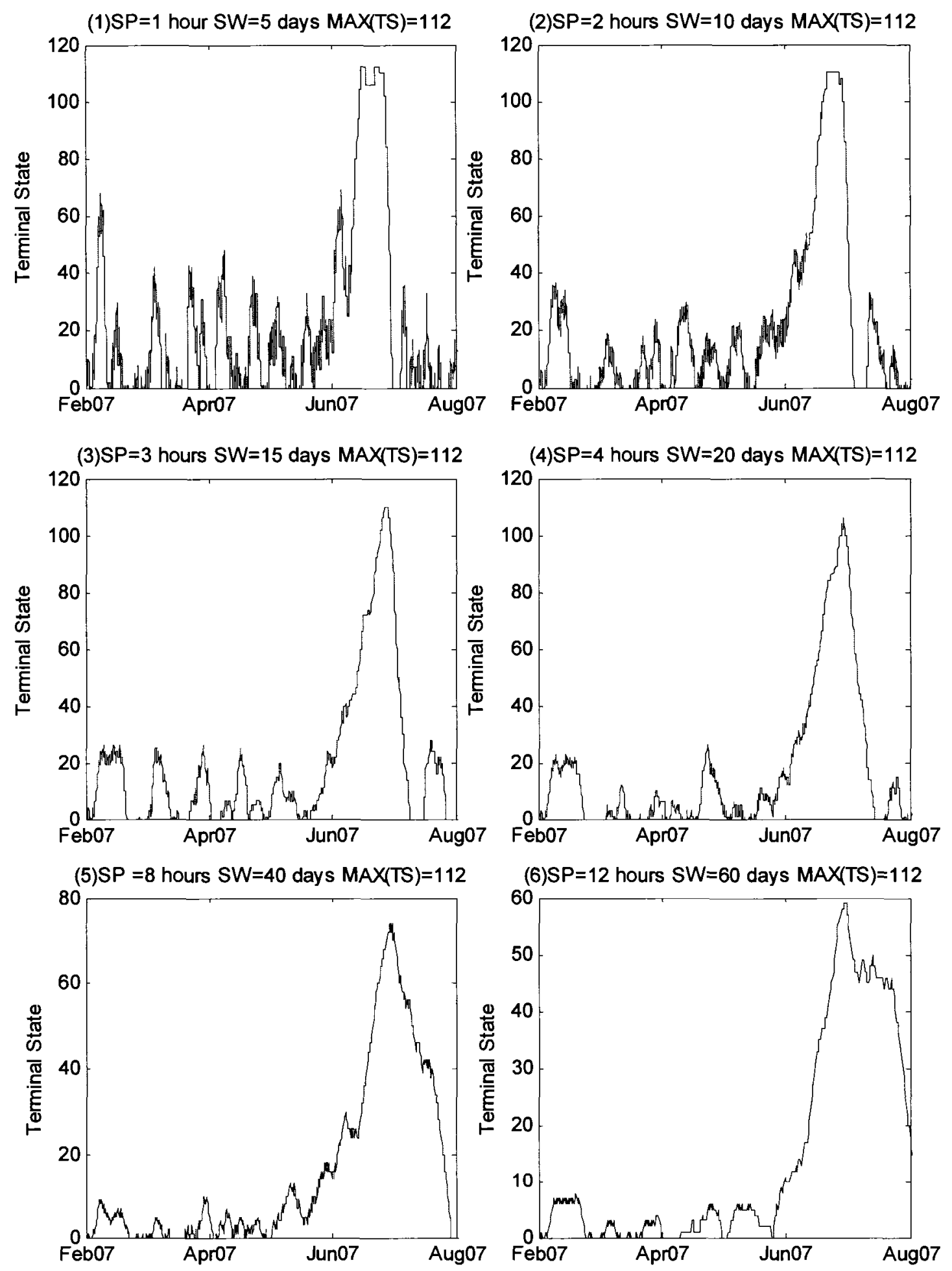

Figure 4.15: Measurement of Terminal State under different SP and SW 


\subsubsection{Single Mode vs. Neighbouring Mode}

In the broadband satellite network, GTs may be deployed close to each other. Neighbouring GTs are usually under the coverage of the same spot beam. They also share the same geographical environment and weather conditions. As a result, the neighbouring terminals should have similar FLS patterns. Cooperating information from neighbouring terminals could minimize biased conclusions. Normally, two GTs are considered to be neighbouring terminals if the distance between them is less then $20 \mathrm{~km}$. The SNR trending algorithm is designed in order to operate under two operation modes, namely the single mode and the neighbouring mode. In the single mode, each GT is isolated. The SNR trending algorithm uses only FLS from the targeted GT in order to generate the TS values. This operation mode is suitable for stand-alone GTs, without the neighbours available for comparative analysis. The neighbouring mode is recommended when there are neighbouring terminals available.

Neighbouring terminals should have a similar FLS most of the time. For short-term performances, when the location is under clear skies, the recorded FLS should be above the expected FLS. If the location experiences a heavy rain/snow-fall rate, then both GTs should record decreased FLS. For long-term performances, if the FLS of the targeted GT drops gradually over time while its neighbouring GT operates under good conditions, then the targeted GT should be considered to be operating in the UNUSUAL situation. If the FLS of both the targeted GT and the neighbouring GT dropped gradually with a similar trend, then the neighbouring terminals may be affected by the same fault source. The system should consider the operating conditions of both GTs as NORMAL. 
In the single mode, the trend of the FLS is stored in the TS. As a result, the TS from the individual terminal represents its own trend. In the neighbouring mode of the SNR trending algorithm, the TS difference is obtained by subtracting the TS of the targeted GT by the TS of the neighbouring GT. The TS difference represents the trend of the FLS for the targeted GT with respect to the trend of its neighbouring GT. If the absolute value of the TS difference is low, this means that the two GTs have similar trends. When the TS difference is a large positive number, this implies that the targeted GT loses its performance while the neighbouring GT is in good condition. The TS difference will have a large negative number if only the neighbouring terminal demonstrates a deteriorating performance.

In this thesis, the operation records of the Terrace station and the Hazelton station are used for verifying the effectiveness of the neighbouring mode. Terrace is located at a latitude of 54.50 north and a longitude of 128.52 west. Hazelton is located at a latitude of 55.25 north and a longitude of 127.67 west. These locations are about $200 \mathrm{~km}$ away from each other. Strictly speaking, they are not neighbouring terminals. However, due to lack of real world examples, these two GTs neighbouring terminals were considered neighbouring terminals. This assumption was approved by Telesat, since they are the closest terminals in the broadband satellite network, and they are both located on the west coast of British Columbia. The Hazelton station was installed in February 2007, and the Terrace station was installed in August 2007. In the experiment, the historical FLS from August 2007 to December 2007 from both stations was used in order to verify the design of the neighbouring mode. Figure 4.16 shows the Terminal State of the Terrace station 
and the Hazelton station from August 1, 2007 to December 31, 2007. The TS of both stations had similar trends from August to October. At the end of October, the TS of the Hazelton station increased fast while the TS of the Terrace station increased slowly. Eventually, the TS of both stations reached their maximum at the end of November. In December, the TS of both stations started to drop, but the TS of the Terrace station dropped faster than that of the TS of the Hazelton station. The change in the TS of these two terminals showed a performance correlation of neighbouring terminals.

When testing the neighbouring mode, the Hazelton station was chosen to be the target GT. The difference of TS shown in Figure 4.17 was obtained by subtracting the TS of the Hazelton station by the TS of the Terrace station. A large positive number of TS difference indicated a deteriorating performance of the Hazelton station. Compared to the single mode, the neighbouring mode correlates with the performance of the neighbouring terminals in order to decide the trends of the targeted GT, and therefore, false alarms are eliminated. A detailed performance comparison of the single mode and the neighbouring mode is given in Chapter 5 . 


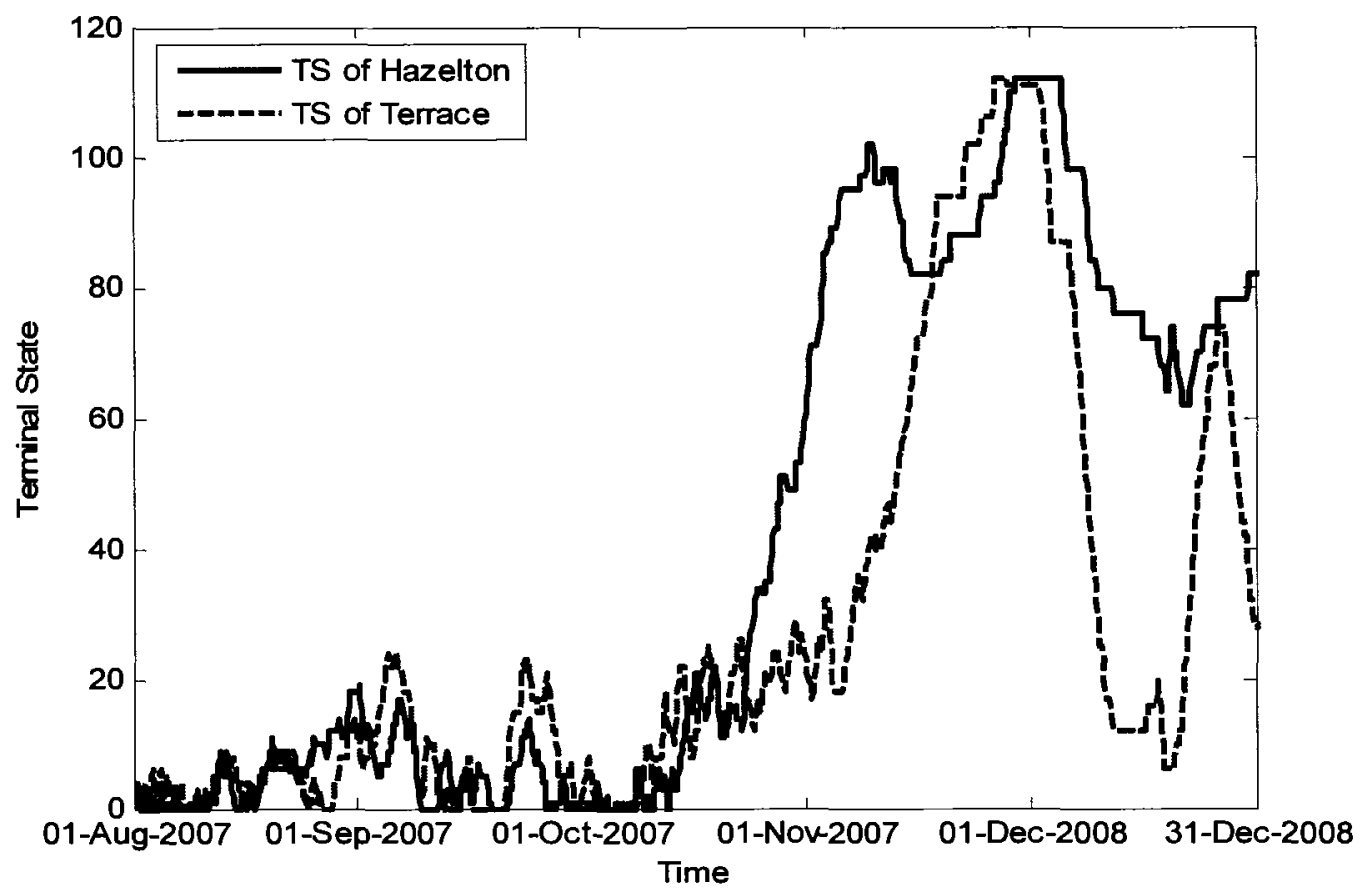

Figure 4.16: Terminal State of Hazelton and Terrace station using single mode

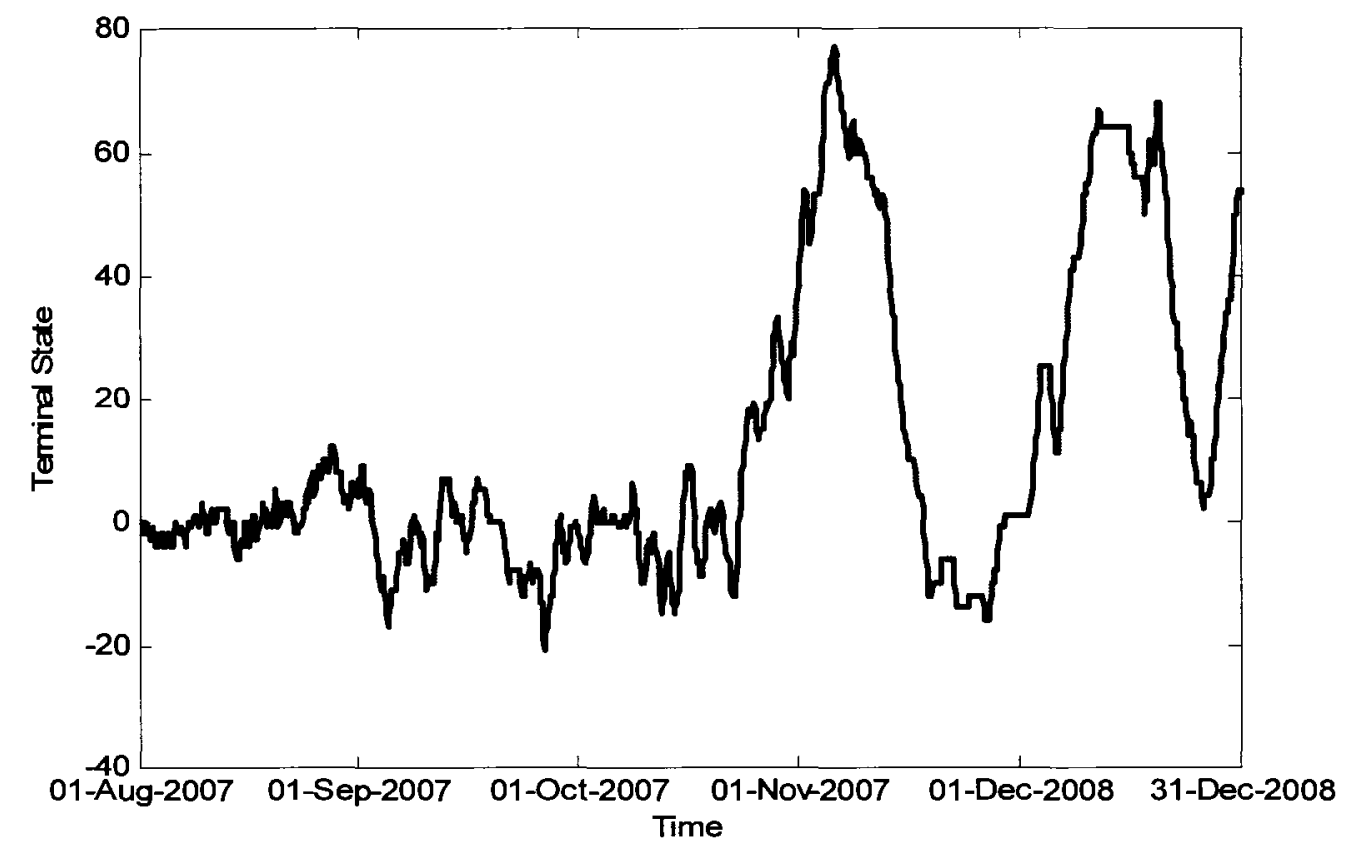

Figure 4.17: TS Difference of Hazelton station using neighboring mode 


\subsection{Maximum Likelihood Decision Making}

The SNR trending algorithm has been tested and analyzed based on the historical FLS samples provided by Telesat. Although the TS provides the time that the GT operates under expected performance during the last $\mathrm{SW}$, the system still needs to select a boundary in order to determine the NORMAL and UNUSUAL conditions. Experienced satellite operators can decide the boundary according to different situations, but it is hard to translate this kind of knowledge into a knowledge base. Choosing a fixed boundary for all the GTs creates bias when making decisions. Therefore, the system needs to find a method in order to make decisions objectively.

According to the analysis of TS, when a GT is operating in the NORMAL condition, the TS trends stay low. When the GT is operating under the UNUSUAL condition, TS tends stay at higher values. Therefore, we assume that there are two different statistical distributions for the TS when the GT is under the NORMAL and UNUSUAL conditions. The Quantile-Quantile (Q-Q) plot is a graphical tool that illustrates the difference between a statistical population and a probability distribution [48]. In this section, the QQ plot is used to verify the statistical distribution of the TS under the NORMAL and UNUSUAL conditions. For each TS generated from the SNR trending algorithm, the probability of the NORMAL and UNUSUAL conditions are calculated based on the probability distribution of each condition. According to the Maximum Likelihood method, if the probability of the NORMAL (UNUSUAL) condition is higher, then the GT is considered operating under the NORMAL (UNUSUAL). 


\subsubsection{Maximum Likelihood Method}

The Maximum Likelihood method has been widely used in various fields. The Maximum likelihood decoding method for detecting wireless signals in digital communication is a well known application of the Maximum Likelihood method [49] [50]. In the knowledgebased TFD system, the Maximum Likelihood method was adapted as a decision method for the NORMAL/UNUSUAL condition using the TS. The detailed design is described below.

When choosing a SP equal to 3 hours and a SW equal to 14 days, the value of the TS varies from 0 to 112 . While GT is operating under the NORMAL condition, the distribution of TS has a small mean. On the other hand, when GT is operating under the UNUSUAL condition, the distribution of the TS has a large mean. Suppose the probability distributions of TS for the NORMAL and UNUSUAL conditions follow the Normal distribution; the hypotheses of the two conditions are illustrated in Figure 4.18. For each given value of TS, the probability of each hypothesis can be calculated. Then, a decision is made towards the hypothesis that has the higher probability. In other words, when the probability distributions of the two hypotheses are given, the decision boundary can be calculated. The decision boundary is where the probability of two hypotheses are equal. As illustrated in Figure 4.18, if the TS falls on the left hand side of the decision boundary, the GT will be diagnosed as NORMAL. On the other hand, if the TS falls on the right hand side of the decision boundary, then the GT will be considered as UNUSUAL. 


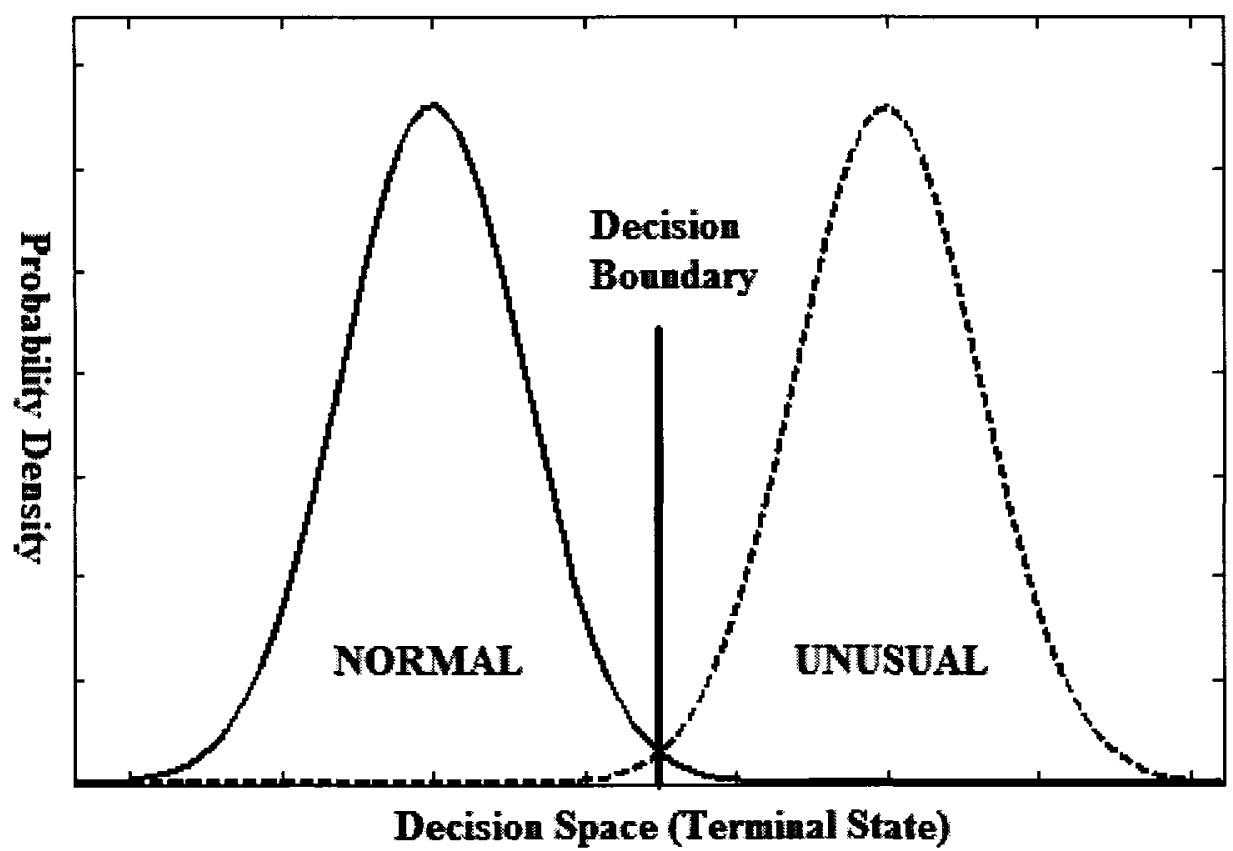

Figure 4.18: Illustrating the hypothesis and decision boundary of NORMAL/UNUSUAL condition

\subsubsection{Distribution Verification}

TSs from the Hazelton station (shown in Figure 4.16 and Figure 4.17) were used to verify the probability distributions of the NORMAL and UNUSUAL conditions for both the single mode and the neighbouring mode. First of all, the TSs are classified into two categories based on their values. Then, Q-Q plot was plotted in order to verify the probability distribution of the TSs in the NORMAL/UNUSUAL condition.

For the single mode, the threshold is set to 40 . Therefore, TSs below 40 belong to the category that represents the NORMAL condition. TSs above 40 belong to the category that represents the UNUSUAL condition. The probability distribution of the NORMAL/UNUSUAL condition is evaluated by means of the Q-Q plot. Figure 4.19 compares the distribution of the TSs under the NORMAL condition with the exponential 
distribution using the Q-Q plot. Figure 4.20 compares the distribution of the TSs under the UNUSUAL condition with Normal distribution using the Q-Q plot. The agreement showed in the figures indicates that the distribution of the NORMAL and UNUSUAL conditions. The summary of the probability distribution of the NORMAL/UNUSUAL condition is shown in Table 4.1. The optimized probability distribution is plotted in Figure 4.21 .

While operating under the neighbouring mode, the TS difference is used to make decisions. The threshold for the NORMAL condition is set to -30 and 30 . If the TS difference is between the thresholds, then it belongs to the category that represents the NORMAL condition. TS differences above 30 are grouped with the category that represents the UNUSUAL condition. Figure 4.22 compares the distribution of the TS difference under the NORMAL condition with Normal distribution using the Q-Q plot. Figure 4.23 compares the distribution of the TS difference under the UNUSUAL condition with Normal distribution. The results show that the distributions of the TS difference under the NORMAL and UNUSAL conditions follow the Normal distribution. The summary of the probability distribution of the NORMAL/UNUSUAL condition is shown in Table 4.2. Figure 4.24 illustrates the probability distributions of the TS difference under the NORMAL and UNUSAL conditions.

According to the Q-Q plot, the distributions of the TS under the NORMAL and UNUSUAL conditions followed the Normal distribution. The means and variances of the NORMAL and UNUSUAL conditions are determined based on the TS that is generated using historical FLS. Although choosing a different threshold for the TS may slightly 
affect the results, this empirical approach gave us a way to obtain the probability distributions using a statistical tool. In addition, if there is a historical FLS available for a GT, it is necessary to pre-estimate the means and variances of TS for the NORMAL and UNUSUAL conditions.

Table 4.1: Probability distributions of NORMAL/UNUSUAL condition under single mode

\begin{tabular}{|l|c|c|c|c|c|}
\hline \multirow{2}{*}{} & \multirow{2}{*}{ Distribution } & \multicolumn{2}{|c|}{ Measured } & \multicolumn{2}{c|}{ Optimized } \\
\cline { 3 - 6 } & & Mean & Variance & Mean & Variance \\
\hline NORMAL & Exponential & 6.77 & 6.77 & 8.00 & 8.00 \\
\hline UNUSUAL & Normal & 81.11 & 12.59 & 80.00 & 15.00 \\
\hline
\end{tabular}

Table 4.2: Probability distributions of NORMAL/UNUSUAL condition under neighboring mode

\begin{tabular}{|l|c|c|c|c|c|}
\hline \multirow{2}{*}{} & \multirow{2}{*}{ Distribution } & \multicolumn{2}{|c|}{ Measured } & \multicolumn{2}{c|}{ Optimized } \\
\cline { 3 - 6 } & & Mean & Variance & Mean & Variance \\
\hline NORMAL & Normal & 0.87 & 9.73 & 0.00 & 15.00 \\
\hline UNUSUAL & Normal & 54.62 & 10.67 & 60.00 & 15.00 \\
\hline
\end{tabular}

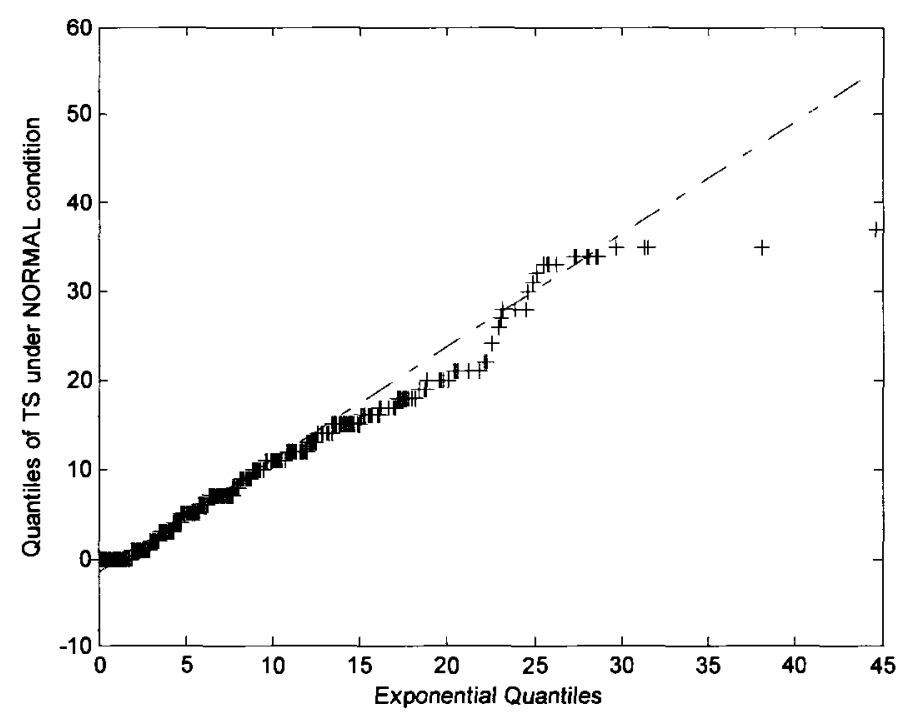

Figure 4.19: Q-Q plot comparing the distribution of TS under NORMAL condition and exponential distribution (single mode) 


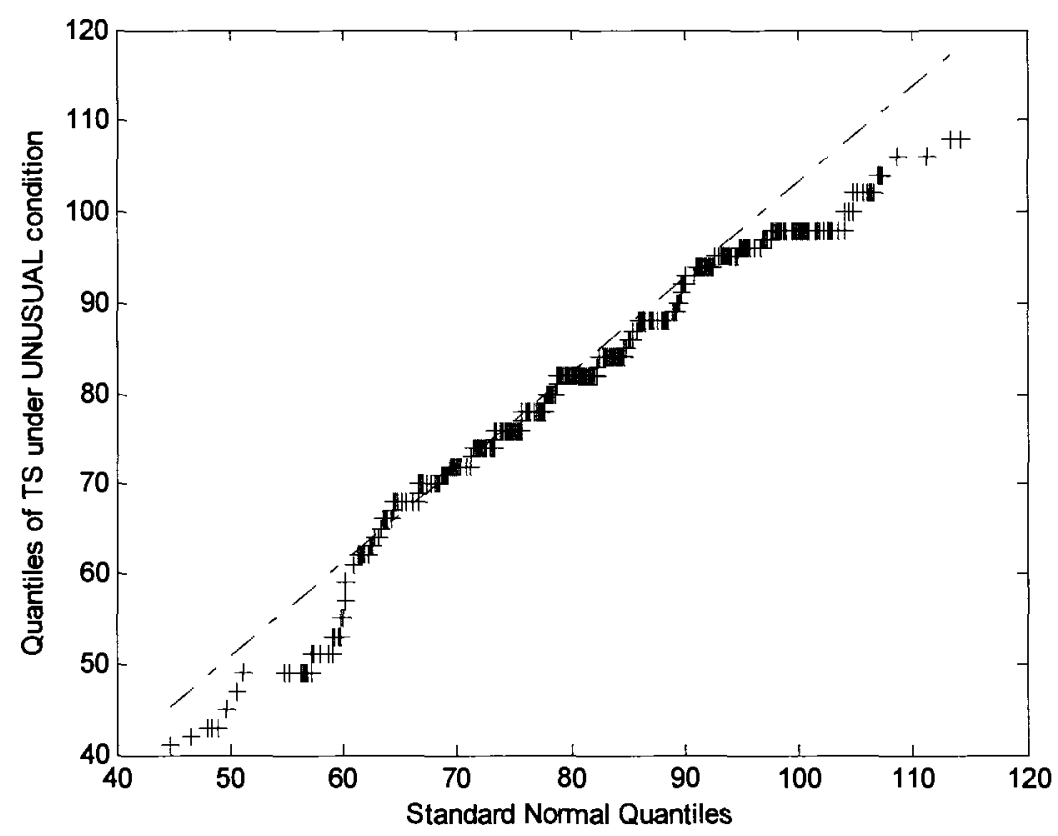

Figure 4.20: Q-Q plot comparing the distribution of TS under UNUSUAL condition and normal distribution (single mode)

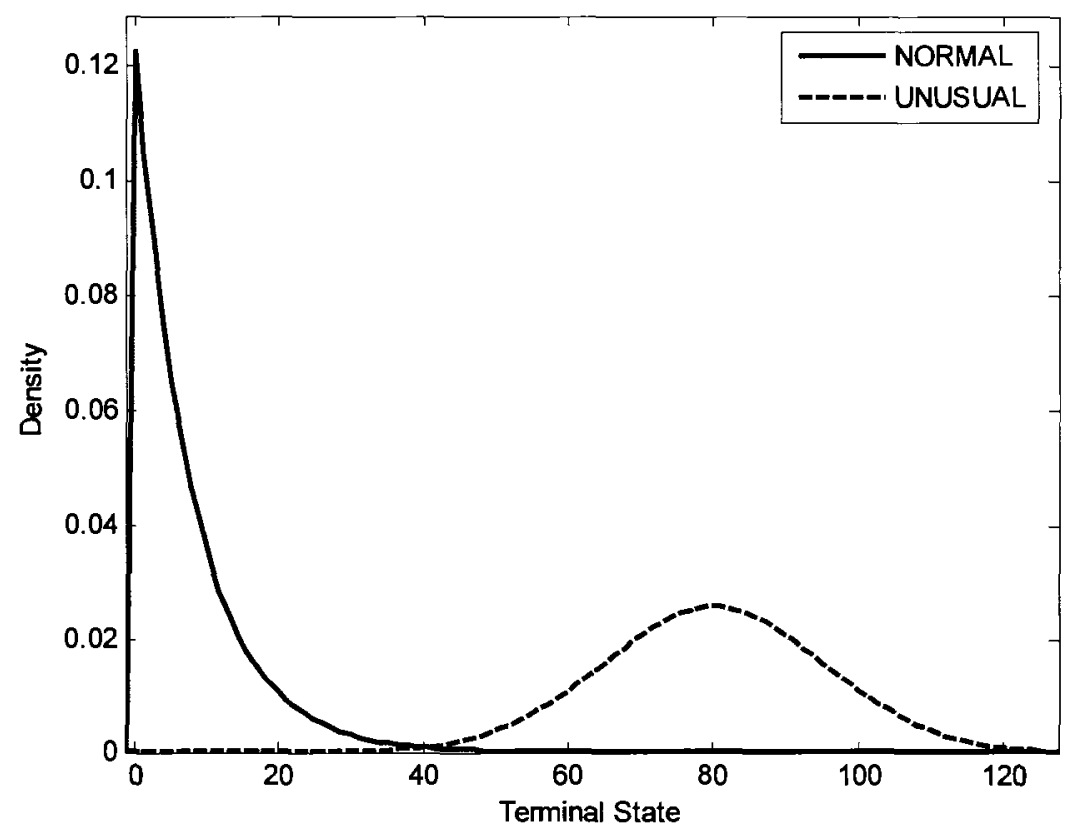

Figure 4.21: Probability distribution of NORMAL/UNUSUAL condition for single mode 


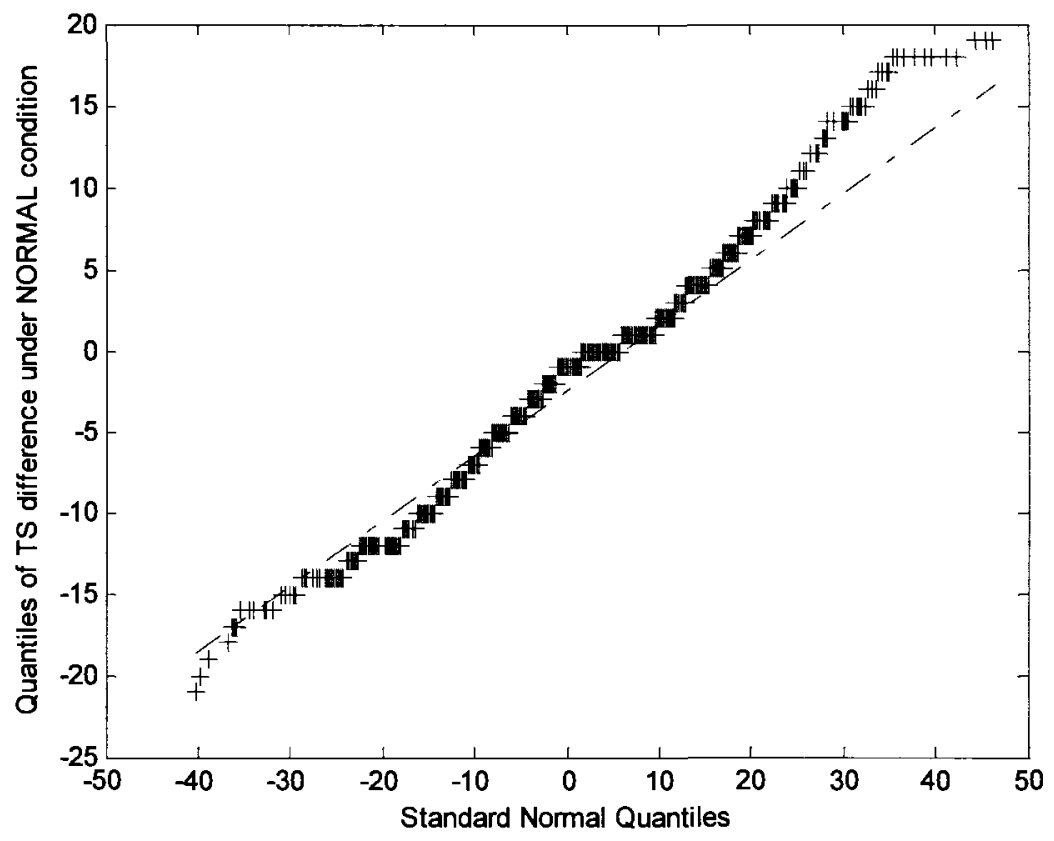

Figure 4.22: Q-Q plot comparing distribution of TS difference under NORMAL condition and normal distribution (neighboring mode)

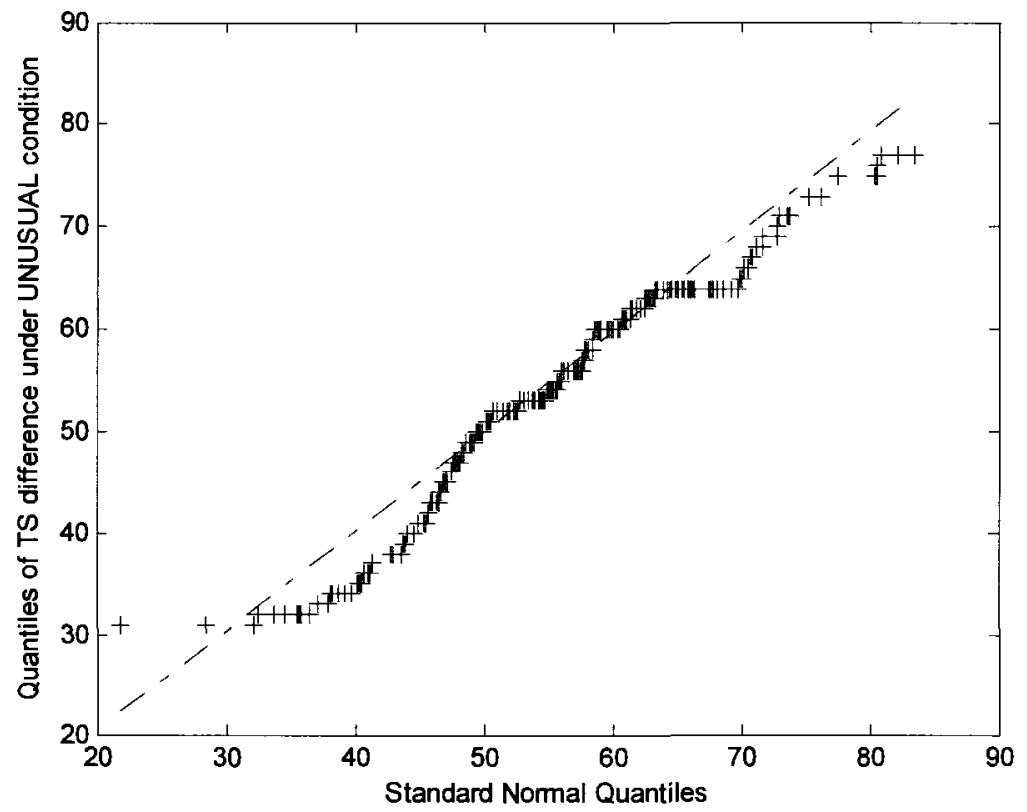

Figure 4.23: Q-Q plot comparing distribution of TS difference under UNUSUAL condition and normal distribution (neighboring mode) 


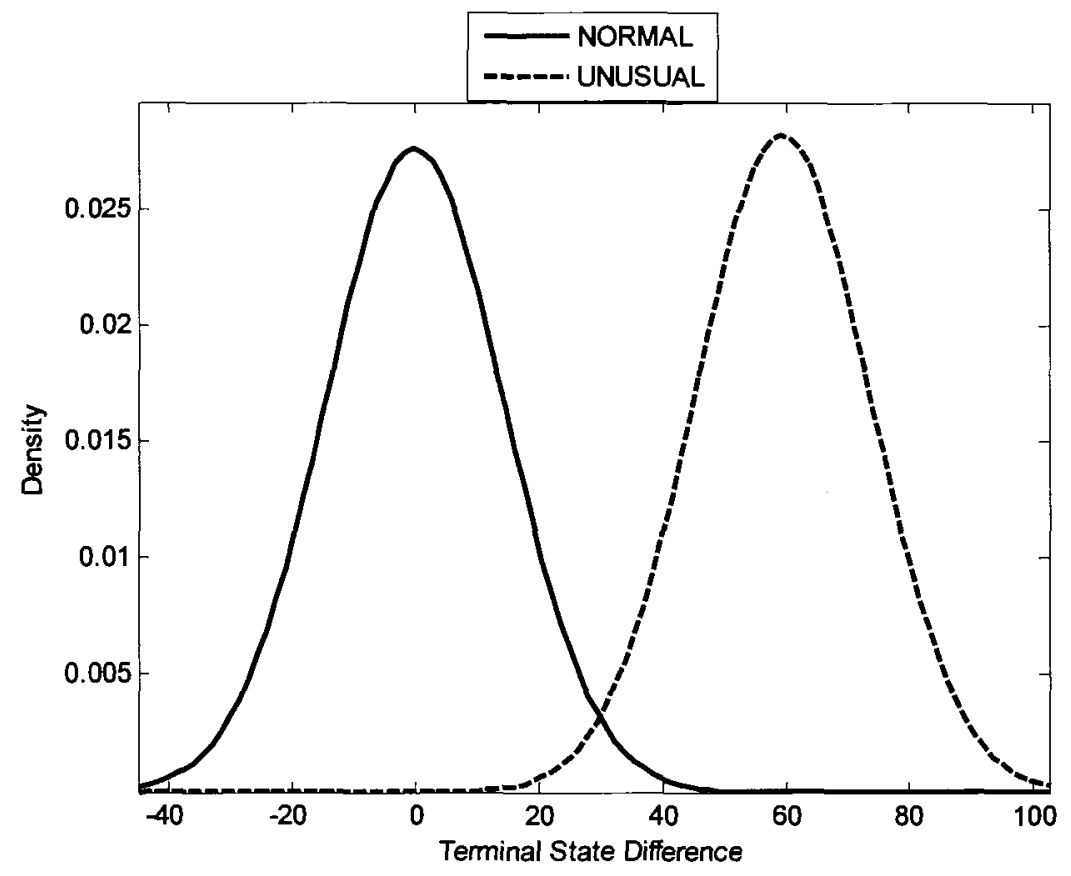

Figure 4.24: Probability distribution of NORMAL/UNUSUAL condition for neighboring mode 


\subsection{Knowledge-based Decision Making}

In the terminal fault diagnosis process, the POWER OFF, CRITICAL, and FLAPPING condition decisions relied heavily on the knowledge of experienced satellite operators. We formulated the human knowledge in a systematic manner using the fuzzy system theory, because the fuzzy system is a rule-based system which allows us to translate human knowledge into rule sets. This section explains the design of the knowledge-based TFD system that is responsible for deciding the POWER OFF, CRITICAL, and FLAPPING conditions.

\subsubsection{FIS Design}

Four indicators generated by the SNR trending algorithm represent the trends of the FLS. They also represent the thinking patterns of experienced satellite operators. Since the NORMAL and UNUSUAL conditions are determined using the Maximum Likelihood method, the FIS is responsible for deciding the POWER OFF, FLAPPING, and CRITICAL conditions. As a result, the FIS has four inputs and three outputs.

The input variables TS, ZC, FI, and $\mathrm{CI}$ are the four indicators generated by the SNR trending algorithm. When $\mathrm{SP}=3$ hours and $\mathrm{SW}=14$ days, the range of input variables are shown in Table 4.3. The outputs of FIS are the POWER OFF, CRITICAL, and FLAPPING indicators. The output variables range from 0 to 10 and stand for the likelihood of the conditions. An output of 0 for POWER OFF means there is no likelihood of the POWER OFF condition. If the output of the FLAPPING condition is 10, then the GT is very likely experiencing a hardware malfunction. However, because the 
center of the gravity defuzzification method is used in FIS, the extreme values 0 and 10 are not achievable. Instead, 1 and 9 are the minimum and maximum values that the system can obtained. The fuzzy sets used to represent each input and output variable are shown in Table 4.3. The FIS uses rule sets to represent human knowledge. A set of rules for each diagnosis symptom was created. The following sections explain how the POWER OFF, FLAPPING, and CIRITCAL conditions are decided using the FIS.

According to our experiences, the shape of the membership functions for each fuzzy set have a small impact on the diagnosis results. However, changing the range of the fuzzy sets will have an effect on the final decisions. On the other hand, changing the number of fuzzy sets that are use to represent a variable will not change the diagnosis results and will not increase the system complexity.

Table 4.3: Input and output variables of FIS

\begin{tabular}{|c|c|c|}
\hline Input Variable & Range & Fuzzy Sets \\
\hline Terminal State (TS) & 0 to 112 & (LOW, MEDIUM, HIGH) \\
\hline Terminal State Difference & -112 to 112 & $\begin{array}{c}\text { ( Left High, Left Medium, Low, Right } \\
\text { Medium, Right High) }\end{array}$ \\
\hline Zero Count (ZC) & 0 to 180 & (Low, Medium, High, Very High) \\
\hline Critical Indicator (CI) & 0 to 16 & (OFF, ON) \\
\hline Fault Indicator (FI) & 0 to 8 & (Low, Medium, High) \\
\hline Output Variable & & (ON, OFF) \\
\hline POWER OFF & 0 to 10 & (Green, Yellow, Red) \\
\hline CRITICAL & 0 to 10 & (Green, Yellow, Red) \\
\hline FLAPPING & 0 to 10 &
\end{tabular}

\subsubsection{Determining POWER OFF condition}

If the number of null data samples recorded during a SP is close to the total data samples in a SP, the GT is said to be POWER OFF. In the FIS, we used ZC in order to decide whether a GT was POWER OFF. If the value of $Z C$ is close to the number of data 
available in the SP, then we assume that the GT is POWER OFF. The membership functions that represent the fuzzy sets of $\mathrm{ZC}$ are shown in Figure 4.25. Based on the percentage of null data recorded, four fuzzy sets were used to represent ZC. They are LOW, MEDIUM, HIGH, and VERY HIGH. In the system, the power is either ON or OFF. Therefore, only two fuzzy sets: ON and OFF were used to represent the POWER OFF Indicator. The membership functions for the POWER OFF indicator are shown in Figure 4.26. The rule set is listed in Table 4.4. The POWER OFF condition is only reported when rule (4) is executed. Figure 4.27 displays the decision curve of the POWER OFF condition. The $\mathrm{x}$-axis represents the $\mathrm{ZC}$, and the $\mathrm{Z}$-axis displays the likelihood of the POWER OFF condition.

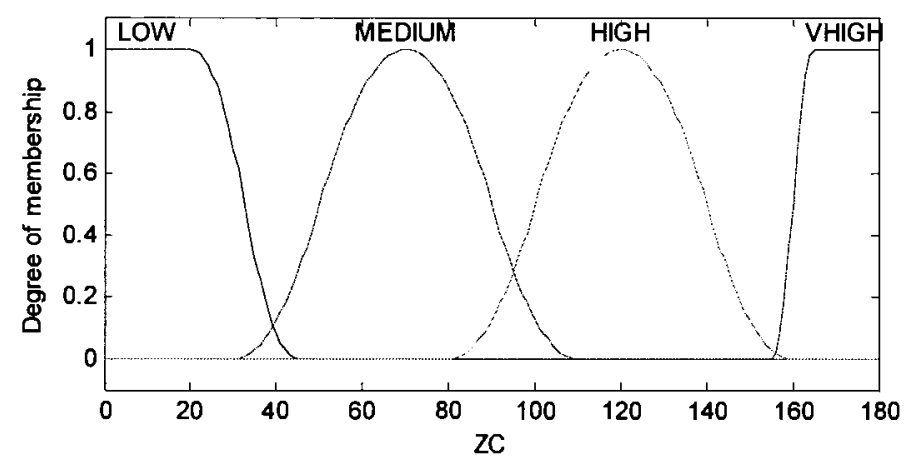

Figure 4.25: Membership functions for $\mathrm{ZC}$

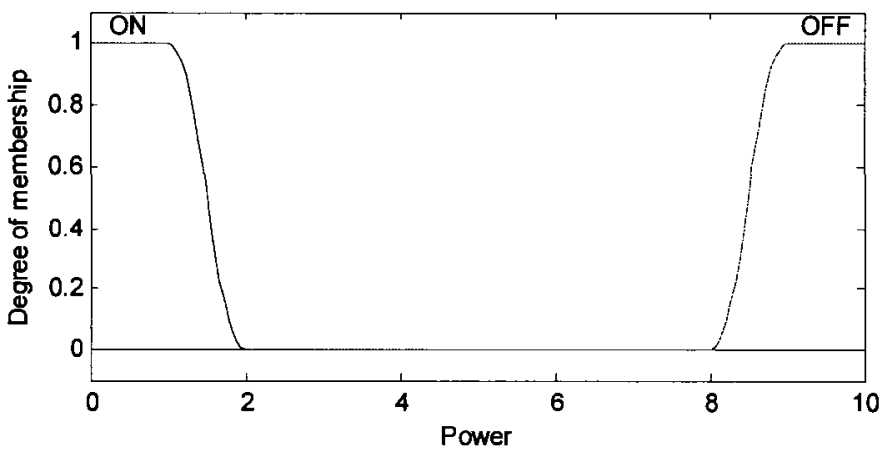

Figure 4.26: Membership functions for POWER OFF Indicator 
Table 4.4: Rule set for diagnosing POWER OFF condition

\begin{tabular}{|c|c|c|}
\hline & Antecedent & Consequent \\
\hline & ZC & POWER OFF Indicator \\
\hline 1 & Low & ON \\
\hline 2 & Medium & ON \\
\hline 3 & High & ON \\
\hline 4 & Very High & OFF \\
\hline
\end{tabular}

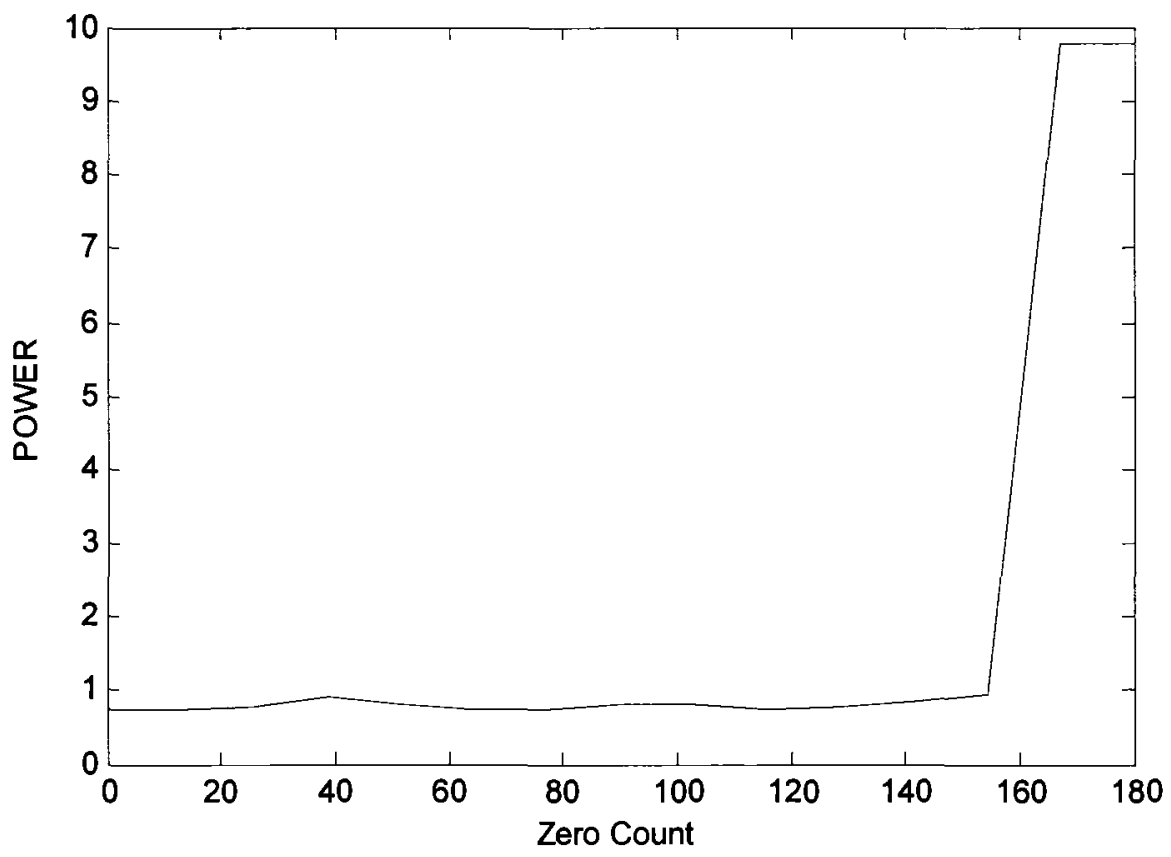

Figure 4.27: Decision curve of diagnosing POWER OFF condition

\subsubsection{Determining FLAPPING condition}

The FLAPPING condition occurs when the FLS shows a fitful pattern continuously throughout the day. The SNR trending algorithm extracts the fitful pattern of the FLS and stores it in FI. Therefore, FI was used as one of the factors in determining the FALPPING condition. In addition, the $\mathrm{ZC}$ was used when making decisions, because $\mathrm{ZC}$ represents the number of null data samples recorded in the current SP. The FI represents the number 
of flapping SPs in the last 24 hours. If the FI is large, then more than likely the GT is under the FLAPPING condition. Three fuzzy sets LOW, MEDIUM, and HIGH were chosen to represent the FI. The membership function for FI is shown in Figure 4.28. Accordingly, three fuzzy sets: GREEN, YELLOW, and RED were used in order to represent the FLAPPING Indicator. The membership functions for the FLAPPING Indicator are illustrated in Figure 4.29. The rule set used to determine the FLAPPING condition is listed in Table 4.5. Figure 4.30 uses a three dimension graph to display the decision surface of the FLAPPING condition. The X-axis and the Y-axis are FI and ZC. The Z-axis displays the likelihood of the FLAPPING condition.

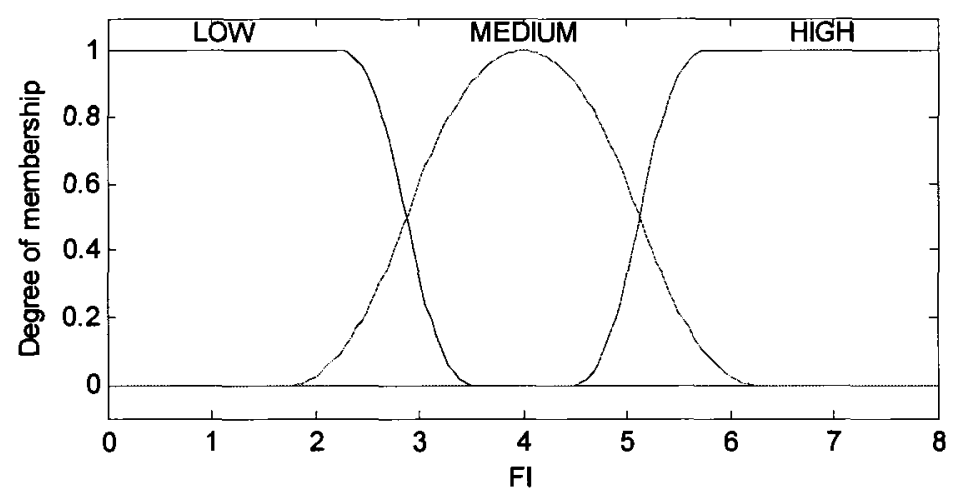

Figure 4.28: Membership functions for FI

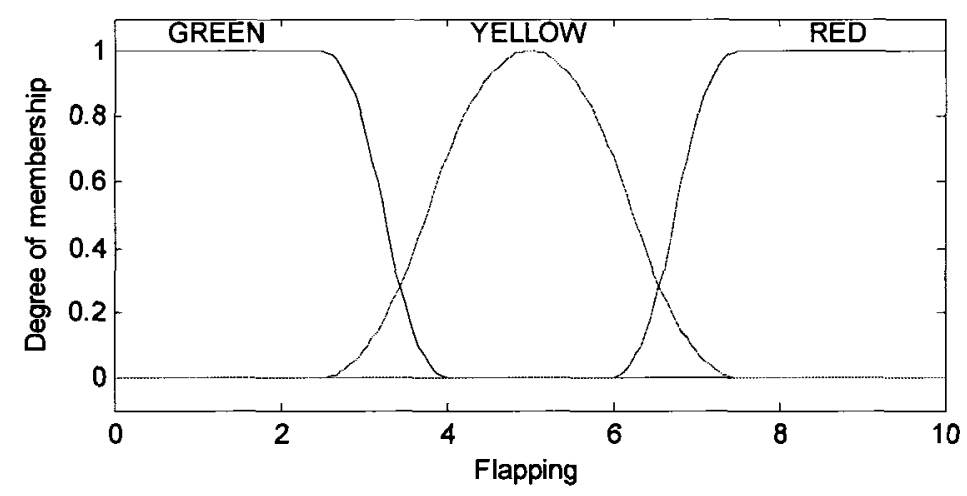

Figure 4.29: Membership functions for FLAPPING Indicator 
Table 4.5: Rule set for diagnosing FLAPPING condition

\begin{tabular}{|c|c|c|c|}
\hline \multirow{2}{*}{} & \multicolumn{2}{|c|}{ Antecedent } & Consequent \\
\cline { 2 - 4 } & FI & ZC & FLAPPING Indicator \\
\hline 1 & LOW & & GREEN \\
\hline 2 & MEDIUM & & RED \\
\hline 3 & HIGH & & RED \\
\hline 4 & LOW & LOW & GREEN \\
\hline 5 & LOW & MEDIUM & YELLOW \\
\hline 6 & LOW & HIGH & RED \\
\hline
\end{tabular}

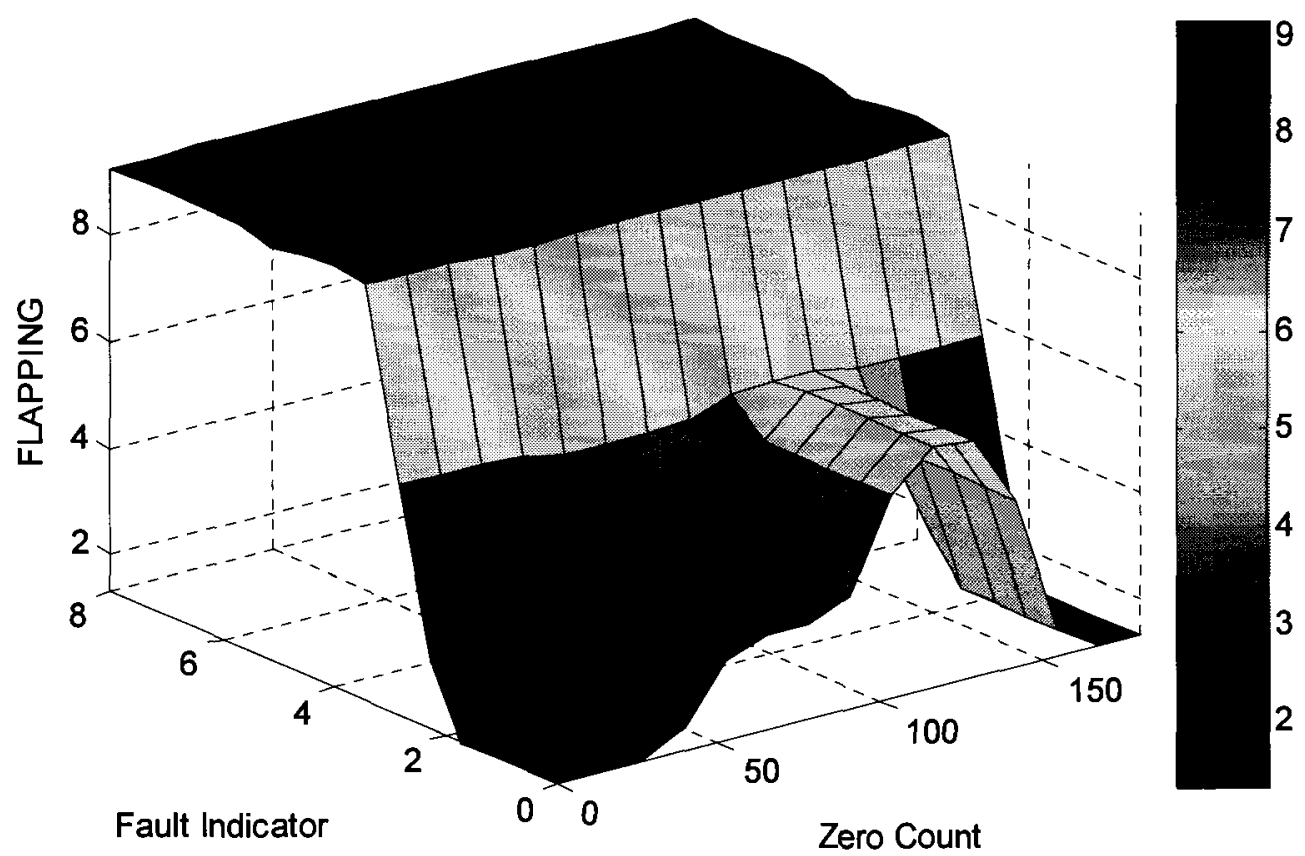

Figure 4.30: Decision surface of diagnosing FLAPPING condition $(x-a x i s=F I, y-a x i s=Z C)$ 


\subsubsection{Determining CRITICAL condition}

When determining the CRITICAL condition more information is needed. First, the system needs to check whether the current GT is operating under the expected performance over a period of two weeks. CI provides the system with this information. If CI is greater than 1 , this means that the GT has already been functioning under the expected performance level for over 2 weeks; therefore, the CRITICAL condition should be reported. If CI is less or equal to 1 , it means that the system is not sure whether the GT is operating under the expected performance; therefore, it needs more information in order to decide whether the GT is under the CRITICAL condition. Other than CI, the satellite operators from Telesat suggested the use of TS and ZC in order to determine the CRITICAL condition. They suggested that if the TS stays at a large number and ZC is high, then the TFD system should report the CRITICAL condition. As a result, TS, CI, and $\mathrm{ZC}$ are used to diagnose the CRITICAL condition.

In order to represent the trends of the FLS in FIS, the fuzzy sets LOW, MEDIUM, and HIGH were used in order to represent the TS. LOW means that the GT is performing under the good condition. MEDIUM means that the GT has a deteriorating trend. HIGH means that the GT is operating under the expected performance for a long period of time. The membership functions for the TS are illustrated in Figure 4.31. Since the system only cares about whether or not the GT has been operating under the expected performance for the last two weeks, two fuzzy sets: ON and OFF were used in order to represent the CI. The membership function for the $\mathrm{CI}$ is shown in Figure 4.32. The rules for determining the CRITICAL condition are listed in 
Table 4.6. Since there are three inputs, the decision surface needs to be drawn in two separate figures. Figure 4.34 illustrates the decision surface of the CRITICAL condition using a three dimension plot. The $\mathrm{x}$-axis and $\mathrm{y}$-axis are $\mathrm{CI}$ and $\mathrm{ZC}$. Figure 4.35 illustrates the decision surface using $\mathrm{ZC}$ as the $\mathrm{x}$-axis and TS as the $\mathrm{y}$-axis. The z-axis in both figures represents the likelihood of the CRITICAL condition.

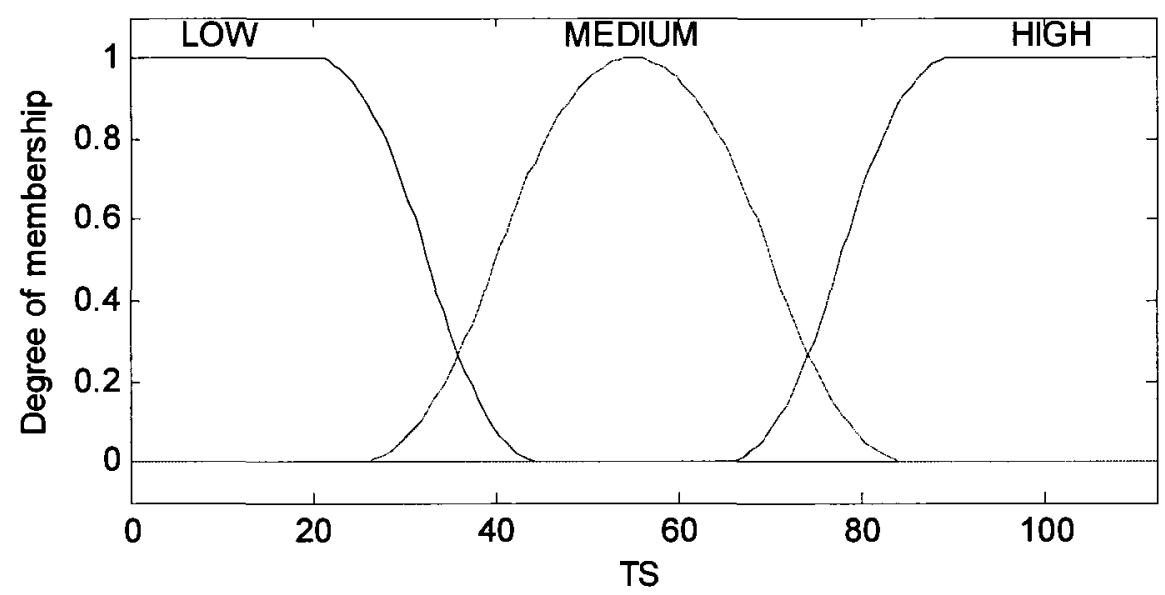

Figure 4.31: Membership functions for TS

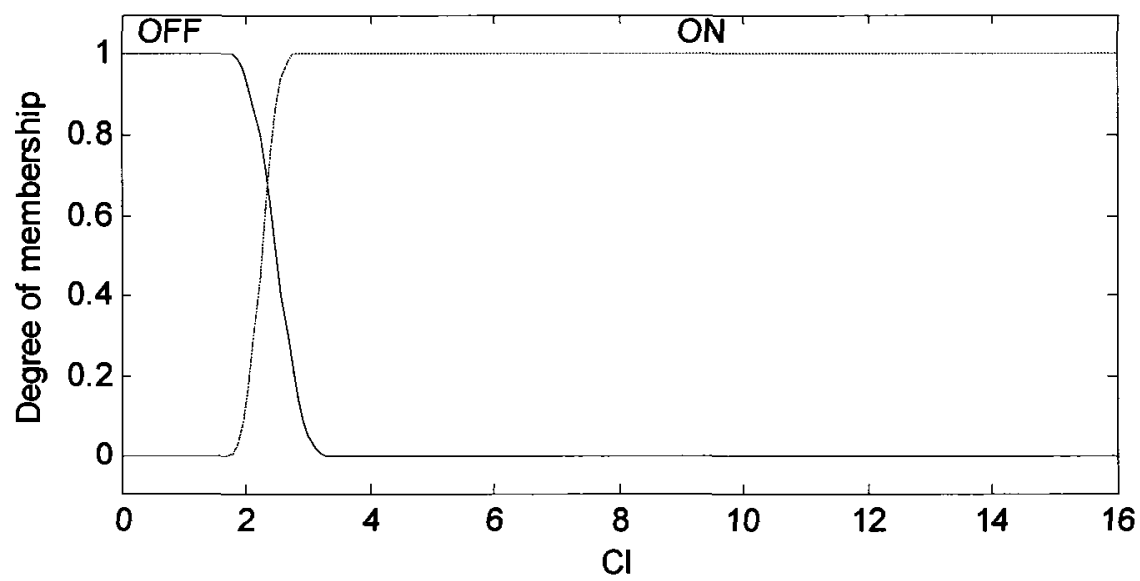

Figure 4.32: Membership functions for $\mathrm{CI}$ 


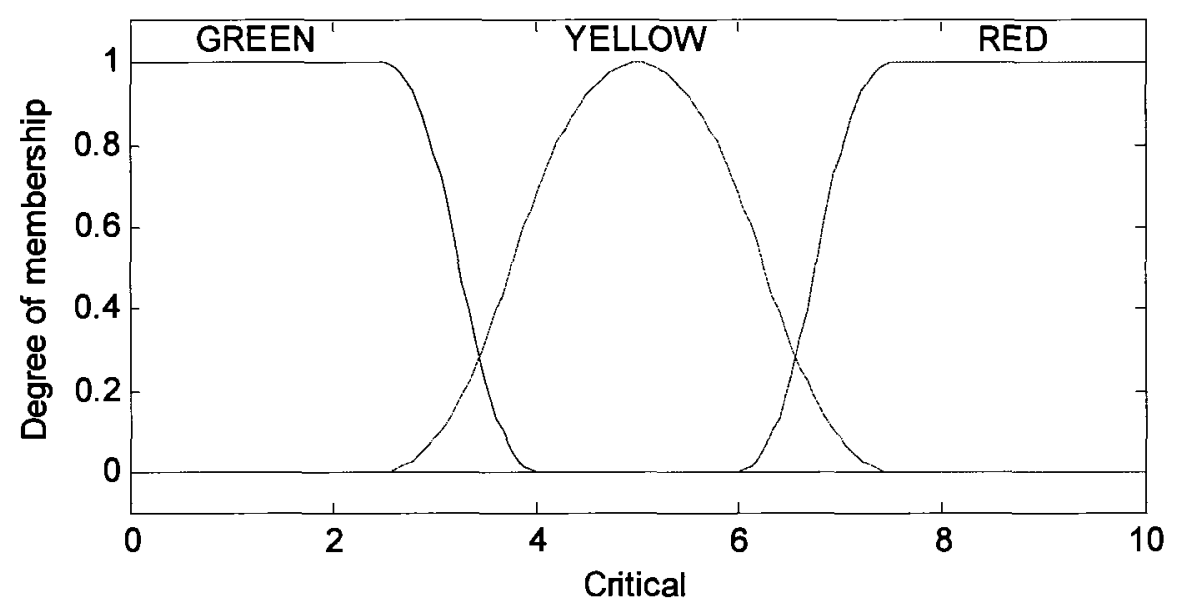

Figure 4.33: Membership functions for CRITICAL Indicator

Table 4.6: Rule set for diagnosing CRITICAL condition

\begin{tabular}{|c|c|c|c|c|}
\hline & \multicolumn{3}{|c|}{ Antecedent } & Consequent \\
\hline & CI & TS & ZC & CRITICAL Indicator \\
\hline 1 & OFF & & & GREEN \\
\hline 2 & ON & & & RED \\
\hline 3 & & MEDIUM & LOW & GREEN \\
\hline 4 & & MEDIUM & MEDIUM & YELLOW \\
\hline 5 & & MEDIUM & HIGH & YELLOW \\
\hline 6 & & MEDIUM & VERY HIGH & GREEN \\
\hline 7 & & HIGH & LOW & GREEN \\
\hline 8 & & HIGH & MEDIUM & YELLOW \\
\hline 9 & & HIGH & MEDIUM & YELLOW \\
\hline 10 & & HIGH & HIGH & RED \\
\hline 11 & & HIGH & VHIGH & RED \\
\hline
\end{tabular}




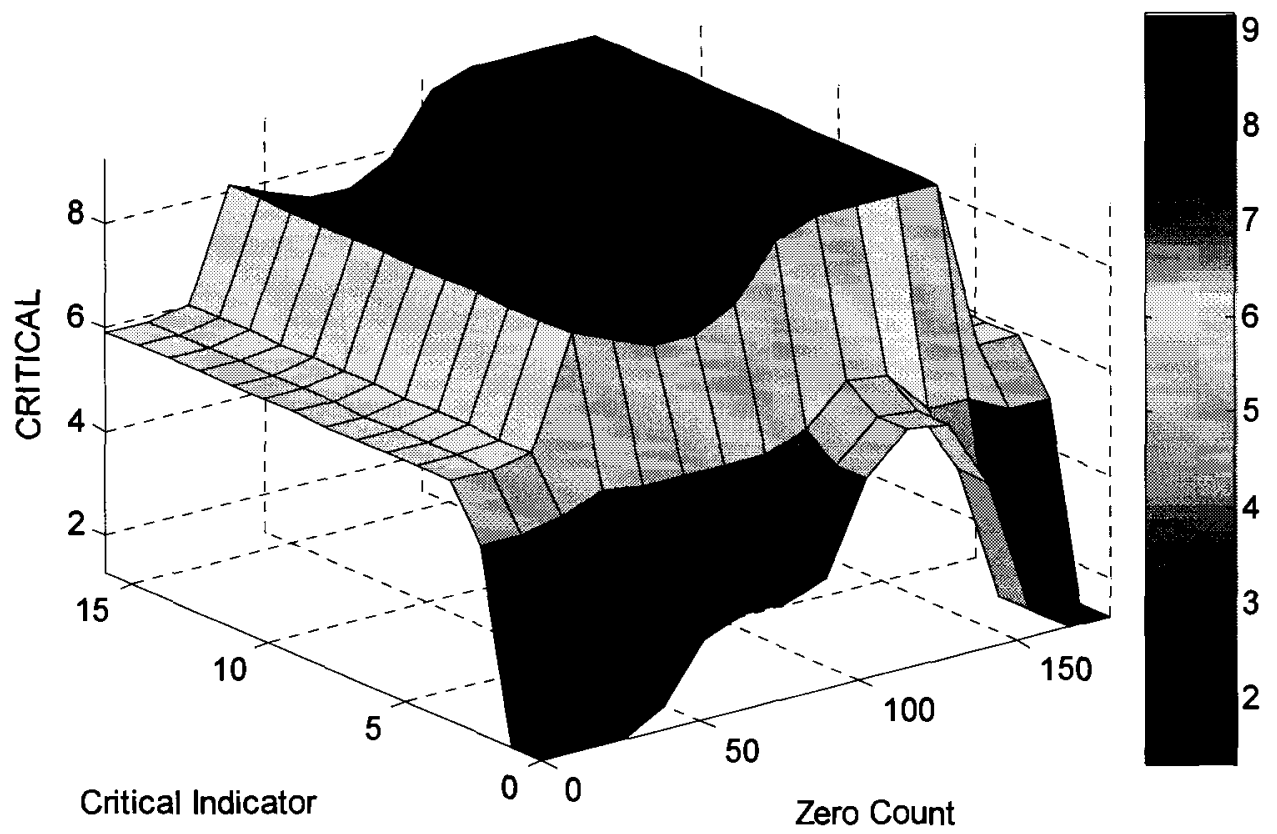

Figure 4.34: Decision surface of diagnosing CRITICAL condition part 1 $(\mathbf{x}$-axis $=\mathrm{CI}, \mathrm{y}$-axis $=\mathrm{ZC}$ )

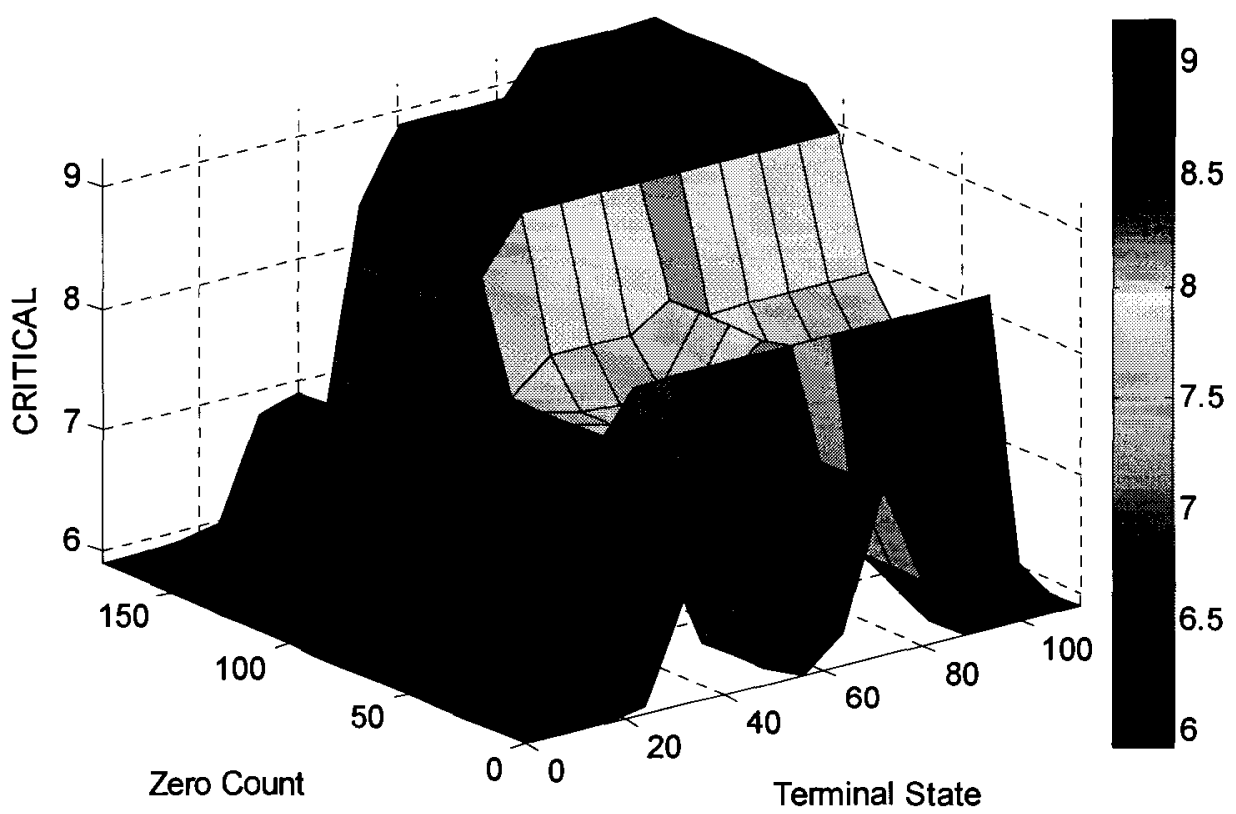

Figure 4.35: Decision surface of diagnosing CRITICAL condition part 2 $(\mathbf{x}$-axis $=\mathbf{Z C}, \mathbf{y}$-axis $=$ TS $)$ 


\subsection{Output Format}

The Knowledge-based TFD system reports the operating condition of the GT every SP. For each SP, the system writes a line of numbers in the text file. The list of output parameters are shown in Table 4.7. The output format is shown in Table 4.8. There are eight fields in the output file. The first column is the index. The index is the sequential arrangement of the diagnosis results. Column 2 and 3 are outputs from the Maximum Likelihood method. They have boolean values and represent whether the operating condition of GT is under the NORMAL or UNUSUAL condition. When the value indicates that the NORMAL condition is equal to 1 , the value represents that the UNUSUAL condition is equal to 0 and vice versa. Columns 4 to 6 are the outputs from FIS; they are the indicators of the POWER OFF, CRITICAL, and FLAPPING condition. They have values from 1 to 9 . Column 6 and 7 are the static day stamp for the diagnosis result. DAY represents the starting day number of the diagnosis. PRD represents the period of the day.

The outputs from the knowledge-based TFD system contain all the information needed in order to diagnose the operating condition of GT. However, only the system developers and experienced satellite operators have the knowledge to make decisions based on the output file. In order to provide a straight forward diagnosis decision of the current operating condition of a GT, a decision making scheme was developed in order to make the final decision. The details of the decision making scheme are described in the next section. 
Table 4.7: Knowledge-based TFD system output parameters

\begin{tabular}{|c|c|}
\hline Output Parameter & Output file Designation \\
\hline Index & INX \\
\hline NORMAL Indicator & NOM \\
\hline UNUSUAL Indicator & UNU \\
\hline POWER OFF Indicator & POW \\
\hline CRITICAL Indicator & CRT \\
\hline FLAPPING Indicator & FLP \\
\hline Day & DAY \\
\hline Period of Day & PRD \\
\hline
\end{tabular}

Table 4.8: Output format of knowledge-based TFD system

\begin{tabular}{|c|c|c|c|c|c|c|c|}
\hline INX & NOM & UNU & POW & CRT & FLP & DAY & PRD \\
\hline 1 & 1 & 0 & 1 & 1 & 1 & 1 & 1 \\
\hline 2 & 1 & 0 & 1 & 1 & 1 & 1 & 2 \\
\hline 3 & 1 & 0 & 9 & 1 & 1 & 1 & 3 \\
\hline
\end{tabular}




\subsection{Decision Constraint}

The output format discussed in the previous section does not give the operating condition of GT directly. It is hard for people who do not have any experience in satellite operation to conclude the current operating conditions of targeted GT. Therefore, a decision making scheme was design in order to conclude the operating condition of the targeted GT. For a better understanding, the conclusion is represented by the name of the five phenomena: NORMAL, UNUSUAL, POWER OFF, CRITICAL, and FLAPPING. Three levels of decision constraints were created in order to analyze the output of the TFD system; these levels are nominal constraint, loose constraint, and tight constraint.

In the nominal constraint, the diagnosis symptom is the dominated phenomenon which is evaluated based on the output value. First, the system compares the output value of the POWER OFF, CRITICAL, and FLAPPING indicators. A conclusion is made based on the highest value among the three indicators. If the values of three indicators are equal, a conclusion will be made in favour of POWER OFF, then CRITICAL, and lastly FLAPPING. If all three indicators are equal to 1 , then the system compares the value of NORMAL or UNUSUAL. If the NORMAL (UNUSUAL) indicator is equal to 1 , the decision will be NORMAL (UNUSUAL).

When using nominal constraint, a conclusion can be made when the likelihood of the phenomenon is low. In order to improve the accuracy of the diagnosis, a threshold is applied in the loose constraint. First, the system selects the dominated phenomenon using the same method used in the nominal constraint. Then, the system compares the value of the dominated phenomenon to a threshold that is equal to 3 . If the value of the dominated 
phenomenon is greater than the threshold, then the system determines that the targeted GT is under the dominated phenomenon. If the value of the dominated phenomenon is less or equal to the threshold, then the system rejects the decision and concludes that the operating condition of the GT is either NORMAL or UNUSUAL. In the tight constraint, a higher threshold is applied. The threshold selected is equal to 5. The large threshold used in the tight constraint helps to eliminate false diagnosis results and increases the false alarm ratio in diagnosing all phenomena. A complete evaluation of the knowledgebased TFD system is given in the next chapter.

Table 4.9 shows three sample outputs of the knowledge-based TFD system. Case (a) is a typical diagnosis result of the NORMAL condition, case (b) is a typical diagnosis result of the UNUSUAL condition, and case (c) is a typical diagnosis result for the FLAPPING condition. These sample outputs demonstrate the effectiveness of different decision constraints.

Table 4.9: Sample outputs of knowledge-based TFD system

\begin{tabular}{|c|c|c|c|c|c|c|c|}
\hline INX & NOM & UNU & POW & CRT & FLP & DAY & PRD \\
\hline 1 & 1 & 0 & 1 & 1 & 1 & 1 & 1 \\
\hline
\end{tabular}

(a) Typical NORMAL condition

\begin{tabular}{|c|c|c|c|c|c|c|c|}
\hline INX & NOM & UNU & POW & CRT & FLP & DAY & PRD \\
\hline 1 & 0 & 1 & 3 & 3 & 3 & 1 & 1 \\
\hline
\end{tabular}

(b) Typical UNUSUAL condition

\begin{tabular}{|c|c|c|c|c|c|c|c|}
\hline INX & NOM & UNU & POW & CRT & FLP & DAY & PRD \\
\hline 1 & 0 & 1 & 5 & 1 & 9 & 1 & 1 \\
\hline
\end{tabular}

(c) Typical FLAPPING condition 
Table 4.10: Diagnosis symptoms for sample outputs under different decision constraints

\begin{tabular}{|c|c|c|c|}
\hline Case & Nominal Constraint & Loose Constraint & Tight Constraint \\
\hline $\mathrm{a}$ & NORMAL & NORMAL & NORMAL \\
\hline $\mathrm{b}$ & POWER OFF & POWER OFF & UNUSUAL \\
\hline $\mathrm{c}$ & FLAPPING & FLAPPING & FLAPPING \\
\hline
\end{tabular}

The diagnosed symptoms of the sample outputs from Table 4.9 under different decision constraints are listed in

Table 4.10. Case (a) showed a typical diagnosis result for the NORMAL condition. Because the POWER OFF, CRITICAL, and FLAPPING indicators stayed at 1 and the NORMAL indicator equalled 1, the operation condition of this GT was NORMAL under all other decision constrains.

Case (b) represented a typical diagnosis result for the UNUSUAL condition. However, in this sample output, the POWER OFF, CRITICAL, and FLAPPING indicators were raised

to 3. This caused a strong interference when making a conclusion about the operating condition of GT. As shown in

Table 4.10, the decision was POWER OFF under nominal constraint and loose constraint and UNUSUAL in tight constraint. When the value of the POWER OFF, CRITICAL, and FLAPPING indicators are equal and/or greater than 1, the decision will be made in favour of the POWER OFF condition. On the other hand, the high threshold 
used in the tight constraint eliminated conditions that have a small likelihood and diagnosed the operating condition as UNUSUAL. This is the expected performance of the original intention of designing the decision constraints.

Case (c) showed a typical FLAPPING condition, because the FLAPPIG indicator reached its maximum value 9. The diagnosis symptom was FLAPPING under all decision constraints.

In this chapter, the design of the proposed knowledge-based system is explained. First, we successfully related the FLS pattern to the operating condition of the satellite GT, and exclusively defined five phenomena that describe the operating conditions of the satellite GT. Each phenomenon is derived by analyzing the trend of FLS using expert knowledge. Second, we created a SNR trending algorithm that extracts FLS trending information from historical FLS. The output parameters of the SNR trending algorithm are the thinking patterns of experienced satellite operators. Third, we used both a statistically decision method and a knowledge-based decision method in order to diagnose the operating condition of the satellite GT. The knowledge-based system is used to diagnose the phenomena that have strong FLS patterns. The Maximum likelihood method is used to diagnose phenomena that have indistinct decision boundary. Our design brought a new diagnosis technique to the terminal fault diagnosis area. In addition, we discovered a way to reproduce the expert thinking pattern using the SNR trending algorithm; furthermore, we discussed how to translate the expert knowledge into rule sets. The knowledge-based TFD system is evaluated based on historical FLS from the Hazelton, Terrance, and the Altin stations. The test results are shown in Chapter 5. 


\section{Chapter 5}

\section{Test Results and Discussions}

\subsection{Testing Environment}

The congestion adaptation system is designed for the broadband satellite network to enhance QoS. The operability of the software models on a real-time broadband satellite network raised a concern. As a result, Telesat developed a broadband satellite test-bed network to evaluate the performance of the congestion adaptation system. The topology of the test-bed network is illustrated in Figure 5.1. It has the same architecture as the broadband satellite network described in Figure 2.1. A satellite channel simulator is used in the test-bed network in order to emulate the RF transmission environment for the broadband satellite signal. In addition, the test-bed network can be remotely accessed from the Internet. In Figure 5.1, the data flow path of the network is displayed by a solid line, and the control signal path is displayed by a dash line. When testing the forward link performance, the traffic source needs to be setup at the development box, and the sink should be setup at the test endpoints. On the other hand, testing the return link performance requires the sink to be setup at the development box and the traffic source to 
be setup at the test endpoint. The detailed test cases and test results for the congestion adaptation system are described and discussed in the next section.

The testing of knowledge-based TFD system required less dependency on the test-bed network configuration compared to the congestion adaptation system. The Knowledgebased TFD system is tested in a stand-alone environment using historical RF information as input. The broadband satellite system generates RF information every minute and stores it in the RF log files. The historical RF information is obtained by extracting RF $\log$ files. A list of available forward link SNR data sets are listed and illustrated in Appendix D. When testing the overall performance of the knowledge-based TFD system, the historical FLS data was provided. When testing the diagnosis of the POWER OFF and FLAPPING conditions, partial FLS data where the symptom had been affirmed were provided.

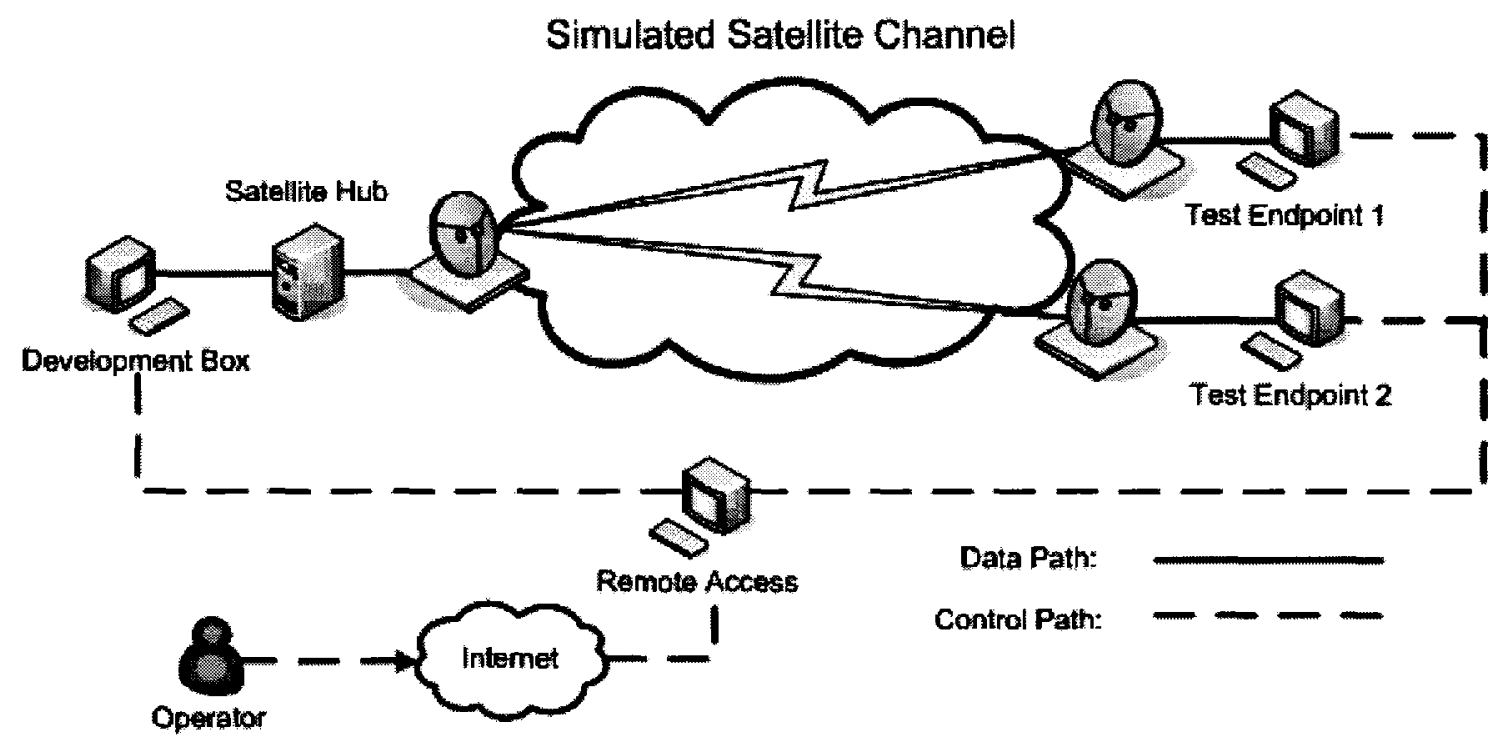

Figure 5.1: Topology of broadband satellite test-bed network 


\subsection{Testing Results for Congestion Adaptation System}

\subsubsection{Testing of Traffic Shaper}

Adapting the maximum traffic rate according to the rain/snow-fall rate is the main objective of the congestion adaptation system. The return link channel was set up in order to test the traffic shaper under different rain/snow-fall rates. The traffic source was located at test endpoint1 where a $1 \mathrm{Mbps}$ UDP traffic stream will be generated during the testing period. Packets are destined for the development box where the measurements are taken. The transmission rate of the return link is calculated base on the number of packets received per second by the development box. The rain/snow-fall rate for the test-bed network is randomly generated by an internal function in the system. The generated rain/snow-fall rate ranges from 0 to 60 and lasted for 30 seconds. For demonstration purposes, five testing results were selected from hundreds of tests. The results are illustrated in Figure 5.2. They showed the transmission rate of the return link channel over a period of 20 seconds. As shown in Figure 5.2, the transmission rate of the return link decreased when the rain/snow-rate increased. The results demonstrated the effectiveness of the congestion adaptation system and the operability of the system on the broadband satellite network. 


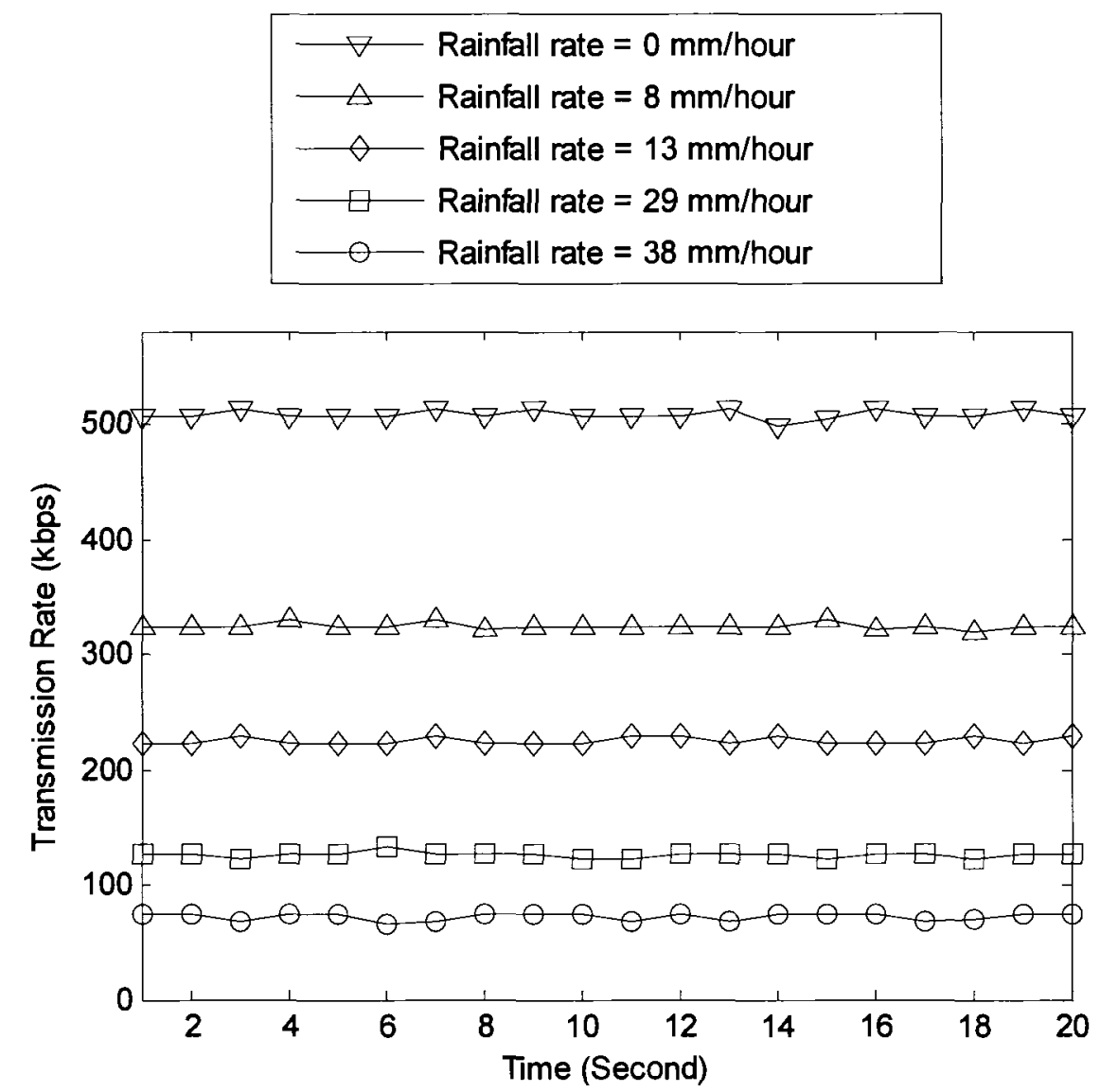

Figure 5.2: Transmission rate of return link under difference rain/snow-fall rate

\subsubsection{Testing of enhanced QoS policy}

The congestion adaptation system has transmission queues for priority and non-priority traffic. It enforces the transmission of priority packets, despite the condition of nonpriority queue. Therefore, the enhanced performance of the priority traffic is at the expense of non-priority traffic. In this section, a return link channel was set up in order to test the enhanced QoS feature of the congestion adaptation system.

Two streams of traffic were generated at test endpoint 1 and sent the streams to the development box. The data rate of the priority traffic stream was $200 \mathrm{kbps}$, and the data 
rate of the non-priority was $512 \mathrm{kbps}$. The transmission rates of priority and non-priority are calculated base on the number of packets that arrived at the development box every second. The performance of the enhanced QoS policy was evaluated under five different transmission rates. The calculated transmission rates of the priority traffic stream and the non-priority traffic stream are shown in Table 5.1. The transmission rate of the priority traffic stayed at $200 \mathrm{kbps}$, the transmission rate of the non-priority traffic decreased, and the transmission rate of the return link decreased. The testing results clearly demonstrated the enhanced QoS policy on the priority traffic stream.

Table 5.1: Transmission rate of priority traffic and non-priority traffic under different transmission rate

\begin{tabular}{|c|c|c|}
\hline $\begin{array}{c}\text { Transmission rate } \\
\text { of return link } \\
\text { (kbps) }\end{array}$ & $\begin{array}{c}\text { Priority Traffic rate } \\
\text { (average) } \\
\text { (kbps) }\end{array}$ & $\begin{array}{c}\text { Non-priority Traffic rate } \\
\text { (average) } \\
\text { (kbps) }\end{array}$ \\
\hline 514 & 200 & 292 \\
\hline 452 & 200 & 223 \\
\hline 396 & 200 & 176 \\
\hline 364 & 200 & 147 \\
\hline 217 & 200 & 0 \\
\hline
\end{tabular}




\subsection{Testing Results for Knowledge-based TFD System}

\subsubsection{Diagnosis of POWER OFF Condition}

As described in the previous chapter, the POWER OFF condition is caused by a power outage or shutting down of the equipment. Figure 5.3 illustrates the FLS pattern of a typical POWER OFF period. During this period, the Hazelton station was shut down by the owner on May 17, 2007. It can be observed that from May 17 to May 18 there was no FLS data recorded. The POWER OFF, FLAPPING, and CRITICAL indicator generated by FIS are plotted in Figure 5.4. The POWER OFF indicator raised and reached its maximum value throughout the POWER OFF period. As a result, the POWER OFF condition was successfully detected. However, at the edge of the POWER OFF period, the FLAPPING indicator reached 2, which indicated a small likelihood of the FLAPPING condition because the knowledge-based TFD system can not distinguish whether the zero data were consecutive zeros or intermissive zeros.

The drawbacks of the system are further investigated using FLS records of the Hazelton station from October 22, 2007 to October 25, 2007. As shown in Figure 5.5, FLS dropped slowly from October $22^{\text {nd }}$ and finally turned into a POWER OFF period on October $23^{\text {rd }}$. The GT experienced another POWER OFF period within a day after it got back online. This strange phenomenon could be caused by a temporary failure of pulling RF data. The POWER OFF and the FLAPPING indicators are illustrated in Figure 5.6. The unusual behavior caused the FLAPPING indicator to increase and reached 3 . The system can overcome this drawback by increasing the decision constraint to tight 
constraint. Nonetheless, there is a chance that the POWER OFF conditions will behave exactly like the FLAPPING conditions and cause the knowledge-based TFD system to misreport.

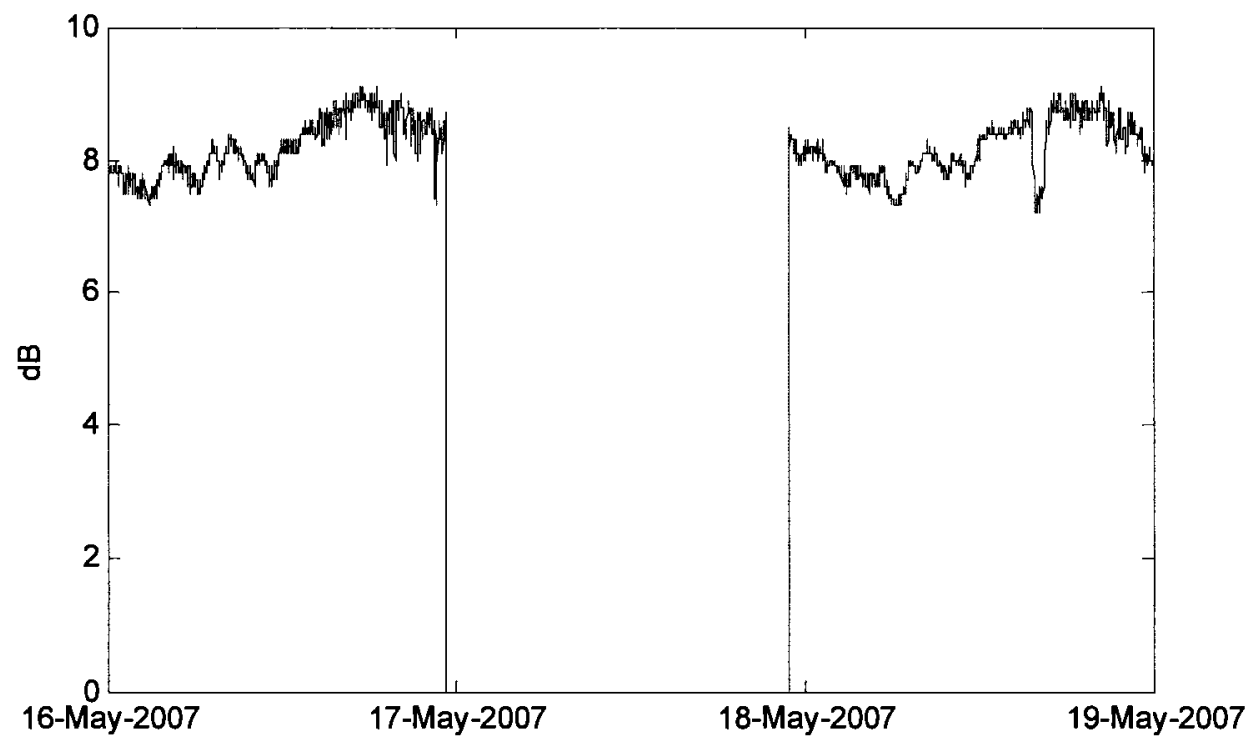

Figure 5.3: FLS of Hazelton station from May $16^{\text {th }} 2007$ to May $19^{\text {th }} 2007$

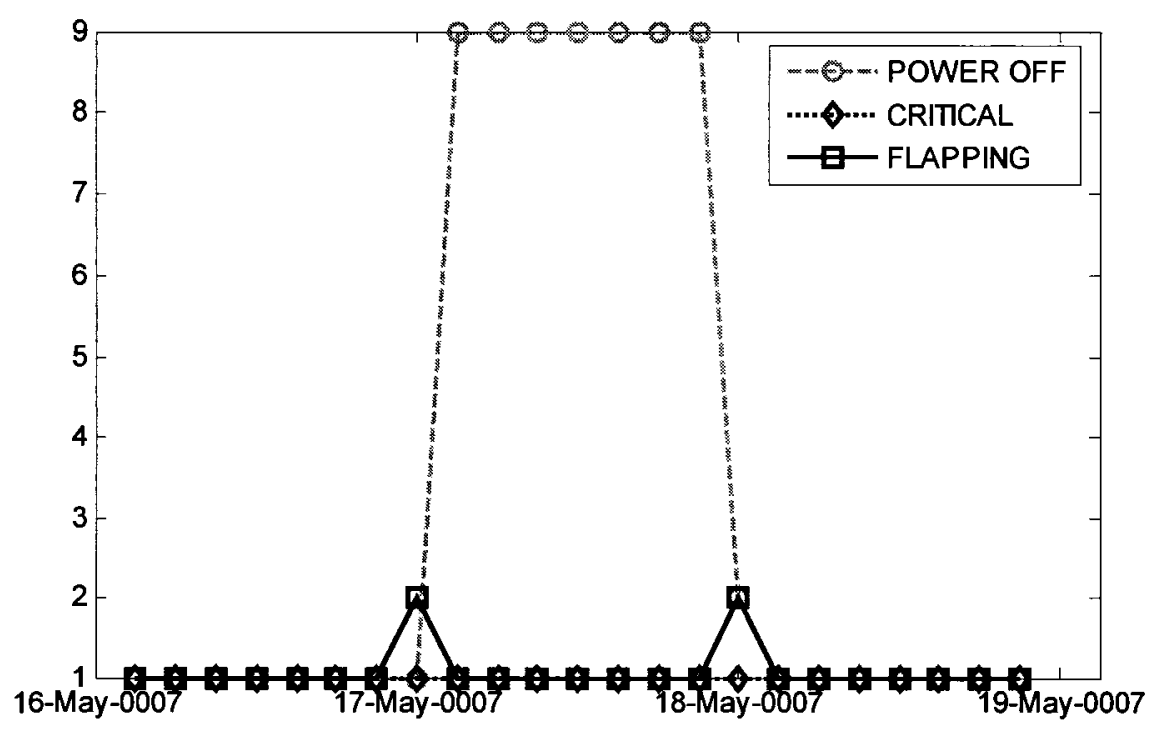

Figure 5.4: Illustrating the output of FIS under POWER OFF condition 


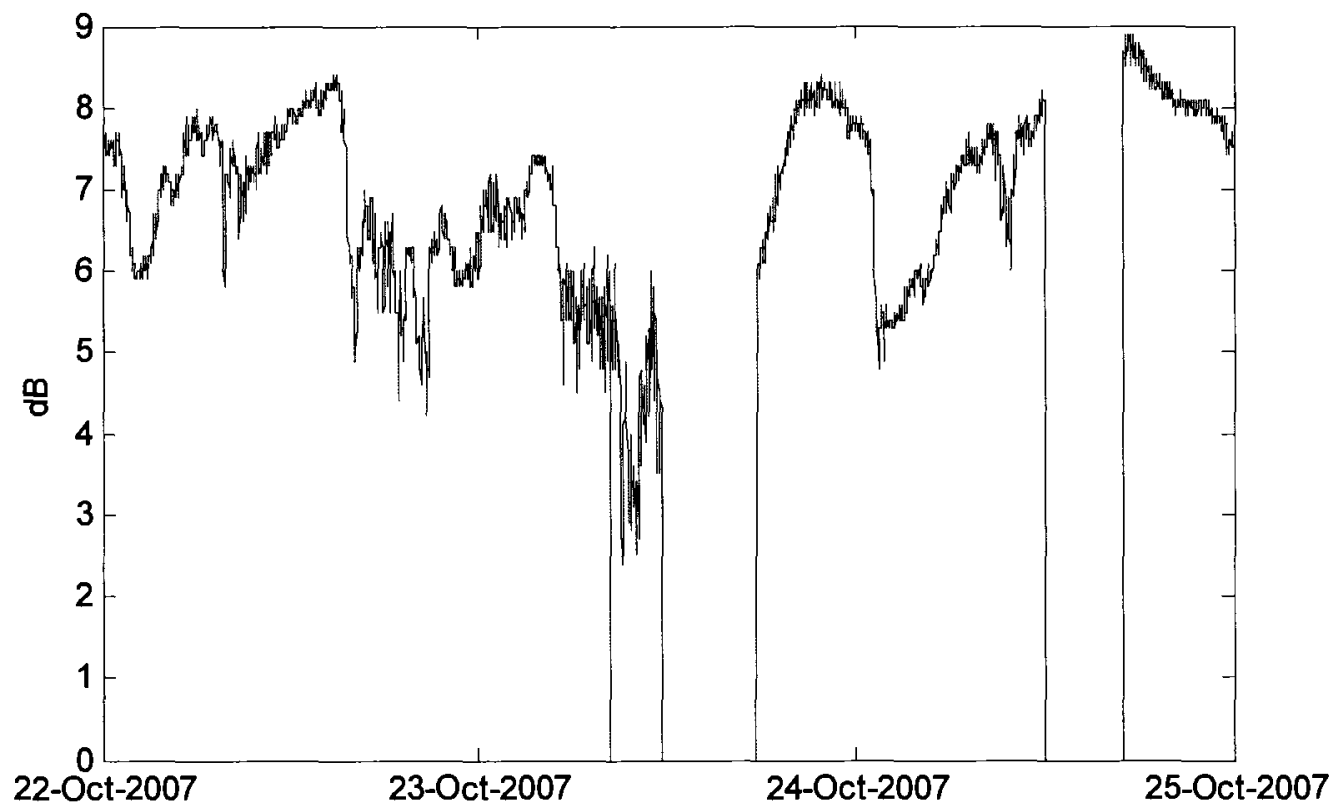

Figure 5.5: FLS of Hazelton station from October $22^{\text {nd }}$ to October $25^{\text {th }} 2007$

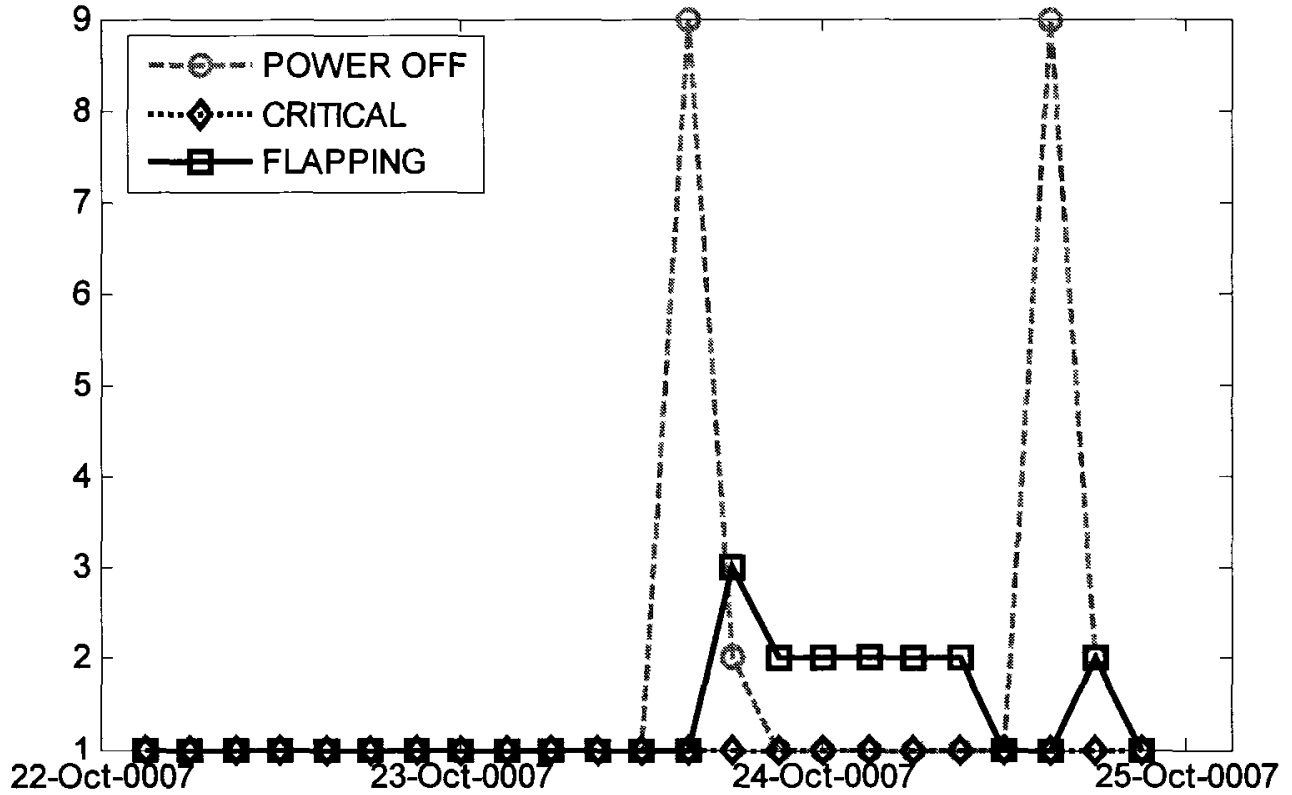

Figure 5.6: Illustrating change of POWER OFF, FLAPPING and CRITICAL indicator 


\subsubsection{Diagnosis of FLAPPING Condition}

The testing of the diagnosis of the FLAPPING condition was performed on FLS of the Atlin station from June 16, 2007 to June 19, 2007. Figure 5.7 shows the FLS during this period. The diagnosis result of the knowledge-based TFD system is illustrated in Figure 5.8. As shown in Figure 5.7, the Altin station had recorded unstable FLS during the two periods on June $17^{\text {th }}$ and $18^{\text {th }}$. The situation was captured by the knowledge-based TFD system since the FLAPPING indicator reached 9. However, the POWER OFF indicator also pointed to 8 during this unstable period. There are two situations that may influence the judgement of the knowledge-based TFD system. First, because the system can not differentiate consecutive zeros and intermissive zeros, the system may misreport the POWER OFF condition when the period contains a large number of zeros. Second, the faulty GT hardware could generates defective RF data samples which failed to be recognized by the knowledge-based TFD system. Therefore, the system could deem the no-response (FLAPPING) periods as POWER OFF conditions. As a result, the knowledge-based system still needs improvement towards the diagnosis accuracy of the FLAPPING condition. 


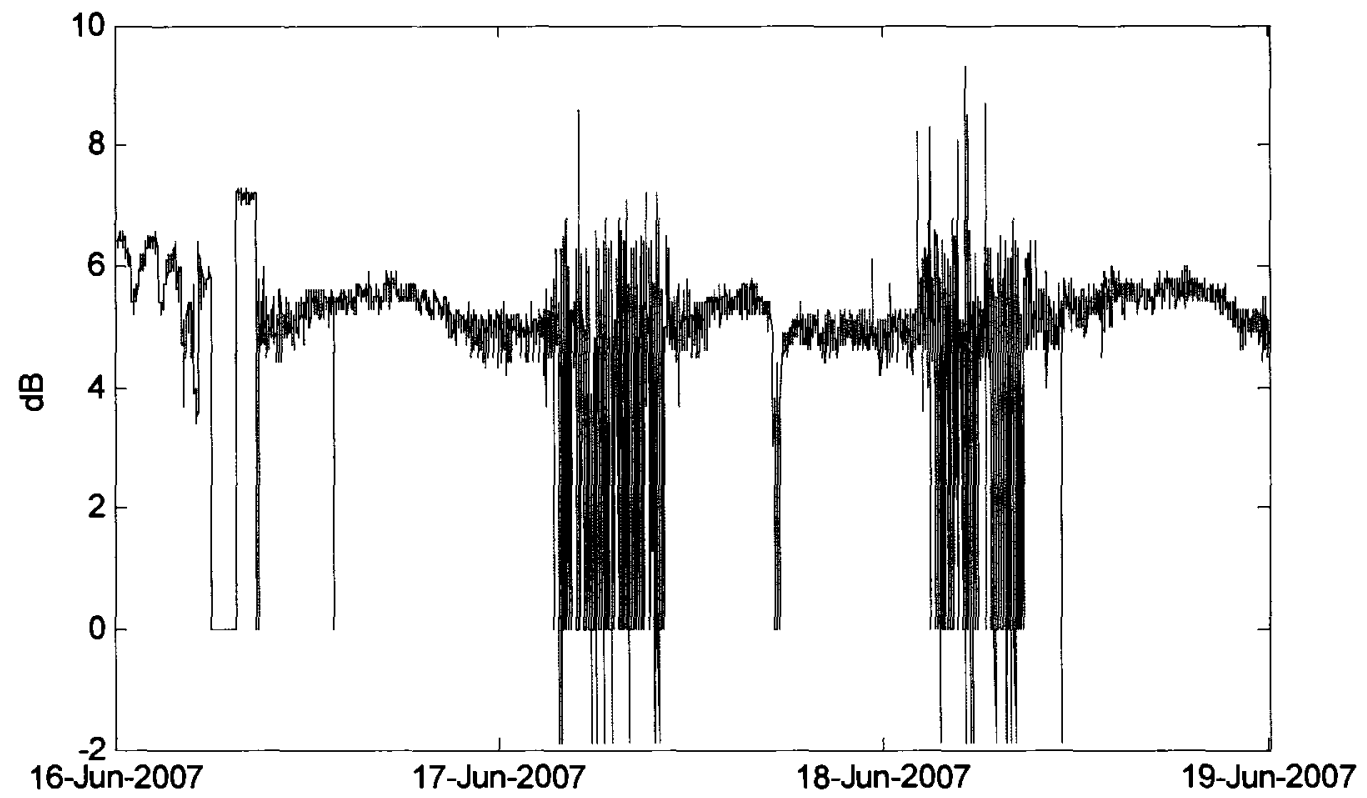

Figure 5.7: FLS of Altin station from June $16^{\text {th }} 2007$ to June $19^{\text {th }} 2007$

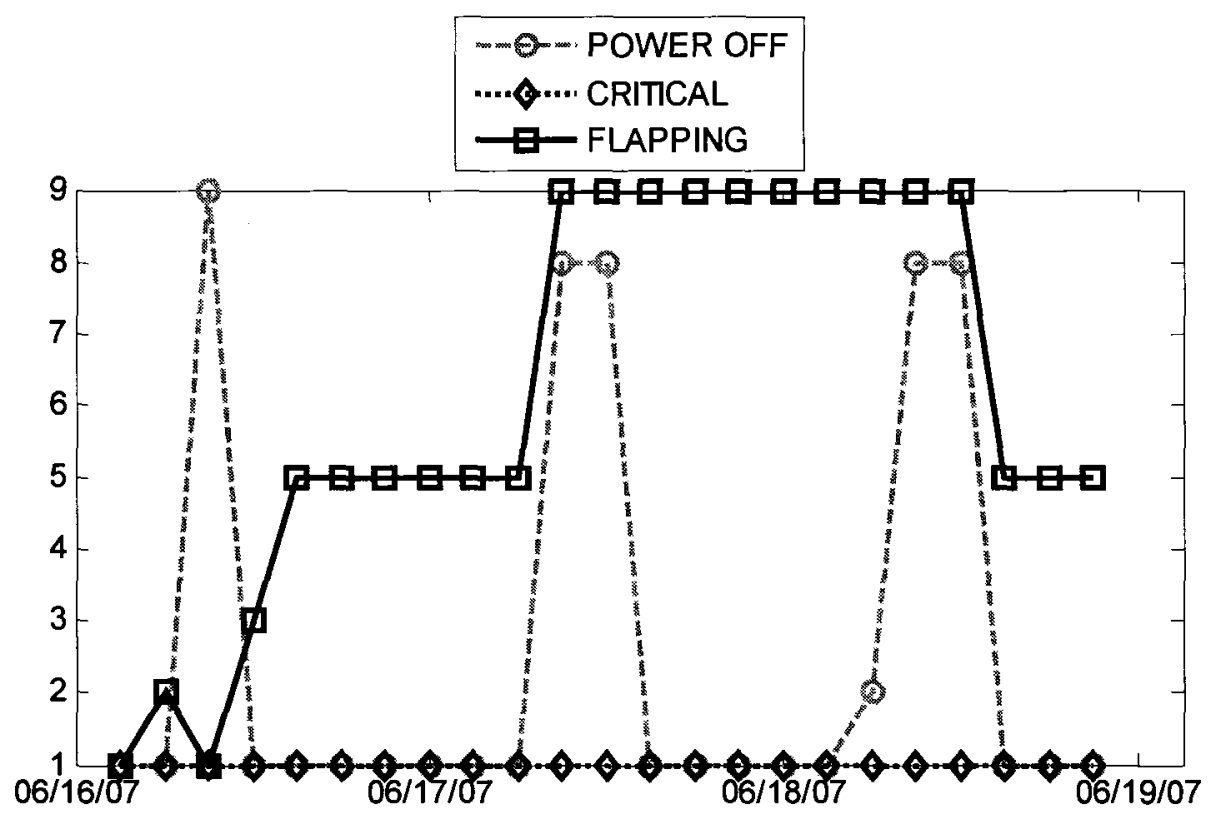

Figure 5.8: Illustrating change of POWER OFF, FLAPPING, INDICATOR indicators 


\subsubsection{Performance of Knowledge-based TFD System under Difference Decision}

\section{Constraints}

The effectiveness of the Knowledge-based TFD system is evaluated using historical RF data collected at the Altin station. Figure 5.9 shows the FLS collected between November 1, 2006 and July 31, 2007 with three hour intervals. The detailed FLS of the Altin station between November 1, 2006 and July 31, 2007 are plotted in Appendix D. Although FLS started low in November, its best performance occurred in March and indicated a FLS fluctuation of around $10 \mathrm{~dB}$. Hence, it is reasonable to set the expected performance of SNR to $9 \mathrm{~dB}$. As illustrated in Figure 5.9, the Altin station was operating below its expected performance from November $1^{\text {st }}$ to late November, but it recovered towards the end of November. The Altin station operated normally from late November 2006 to mid April 2007. During this period, FLS stayed above the expected performance most of the time and dropped below the $9 \mathrm{~dB}$ threshold for a short amount of time in late December and early February. A power off period during late January is clearly illustrated in the figure by consecutive measurement of zero FLS. The operating condition of the Altin station started to deteriorate in mid April 2007 since the FLS of the Altin station dropped $2 \mathrm{~dB}$ and stayed low ever since. The FLS took a further step down in June and started disruption frequently in June. Finally, the Altin station went out of service in July. This is a real case provided by Telesat. The on-site investigation of the Altin Station revealed the secret behind the deteriorating performance. After an on-site investigation, Telesat confirmed that the step drop of FLS at the Altin station was caused by a shifted antenna. The antenna strayed away from its original position due to a loose screw at the junction 
that was supposed to secure the antenna on the roof. The unexpected change in the antenna's location brought an extra load onto the receiving and transmitting devices of the station and undermined the hardware of the Altin station. As the deteriorating performance continued, a hardware malfunction occurred in June. The station became highly unstable and provided low quality service when it was under the hardware malfunction. After struggling for a month, the faulty hardware was replaced by new equipment, and the antenna was reinstalled in a new position with a solid junction.

The fault diagnosis of the Altin station was performed with a targeted SNR equal to 9 $\mathrm{dB}$. The diagnosis results under the tight constraint for the Altin station are plotted in Figure 5.10. Table 5.2 displays the percentage of time of each operation condition under the different decision constraints. Figure 5.11 illustrates the information listed in Table 5.2 using a pie chart. As shown in the diagnosis results, the Altin station operated under the NORMAL condition around $57 \%$ of the time under different decision constraints. The periods of the NORMAL condition are clearly shown in Figure 5.10, and it accurately reflects the operating condition of the Altin station during those periods. Diagnosis of the POWER OFF period under different decision constraints yielded similar results. This indicated the accuracy diagnosis of the POWER OFF condition. As illustrated in Figure 5.10, the POWER OFF period in late January is reported correctly. The FLAPPING periods can be addressed in Figure 5.10 where the FLAPPING conditions are expected. The Knowledge-based TFD system requires an initialization period before it can detect the UNUSUAL condition. The initialization period can be observed in Figure 5.10 where the knowledge-based TFD system indicated the NORMAL condition despite the fact that 
the Altin station was operating under expected performance levels in November. After the initialization period, the knowledge-based TFD system successfully detected the underperformed periods in early November 2006.

In conclusion, using different decision constraints resulted in different diagnosis results. Nominal constraint deals with the raw outcome of the knowledge-based TFD system, but may misreport the POWER OFF, FLAPPING, and CRITICAL conditions. Using loose constraint reduces the misreports on the POWER OFF, FLAPPING, and CRITICAL conditions. Tight constraint utilizes the large decision threshold in order to minimize the misreports on the POWER OFF, FLAPPING, and CRITICAL conditions. As a result, tight constraint is recommended when performing a terminal fault diagnosis with a knowledge-based TFD system. 


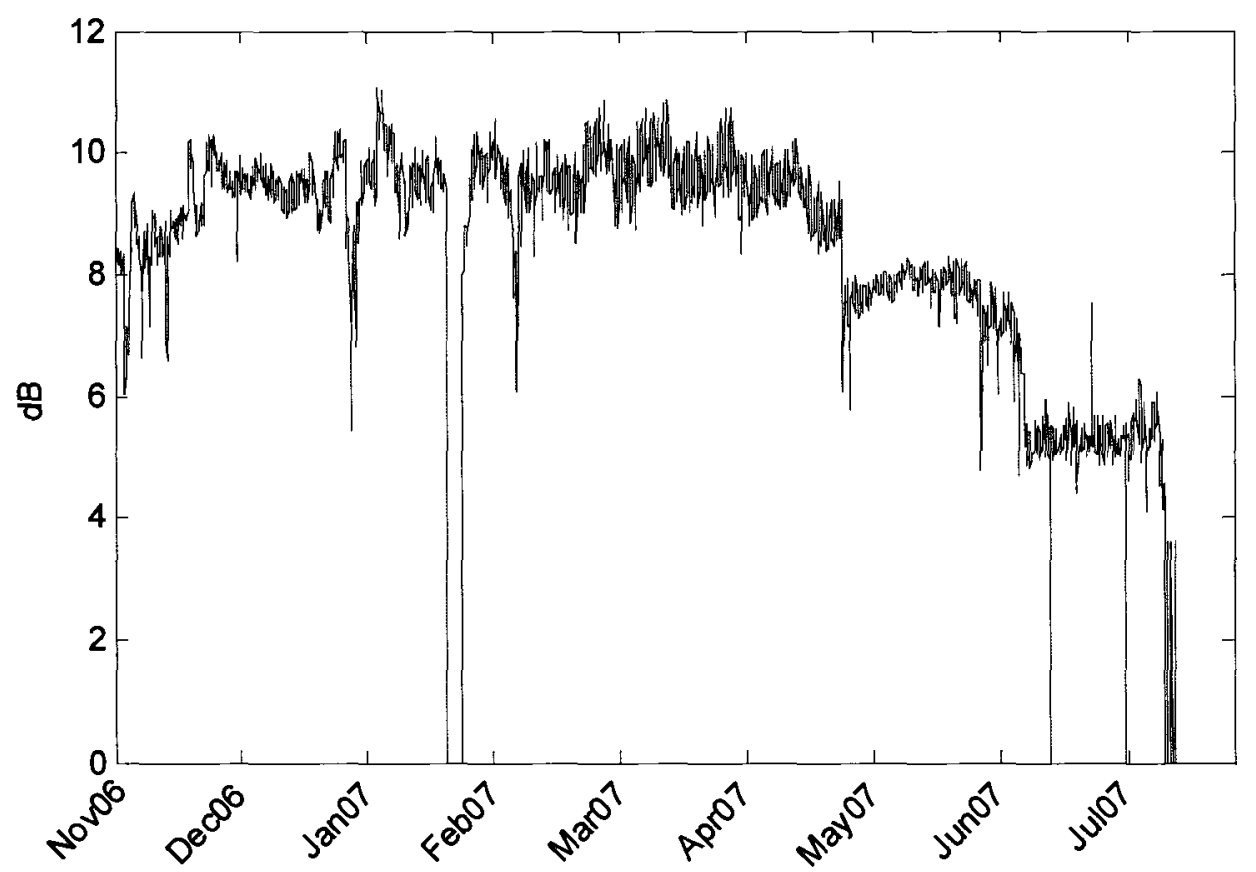

Figure 5.9: FLS of Altin station from November 2006 to July 2007 (3 hour average)

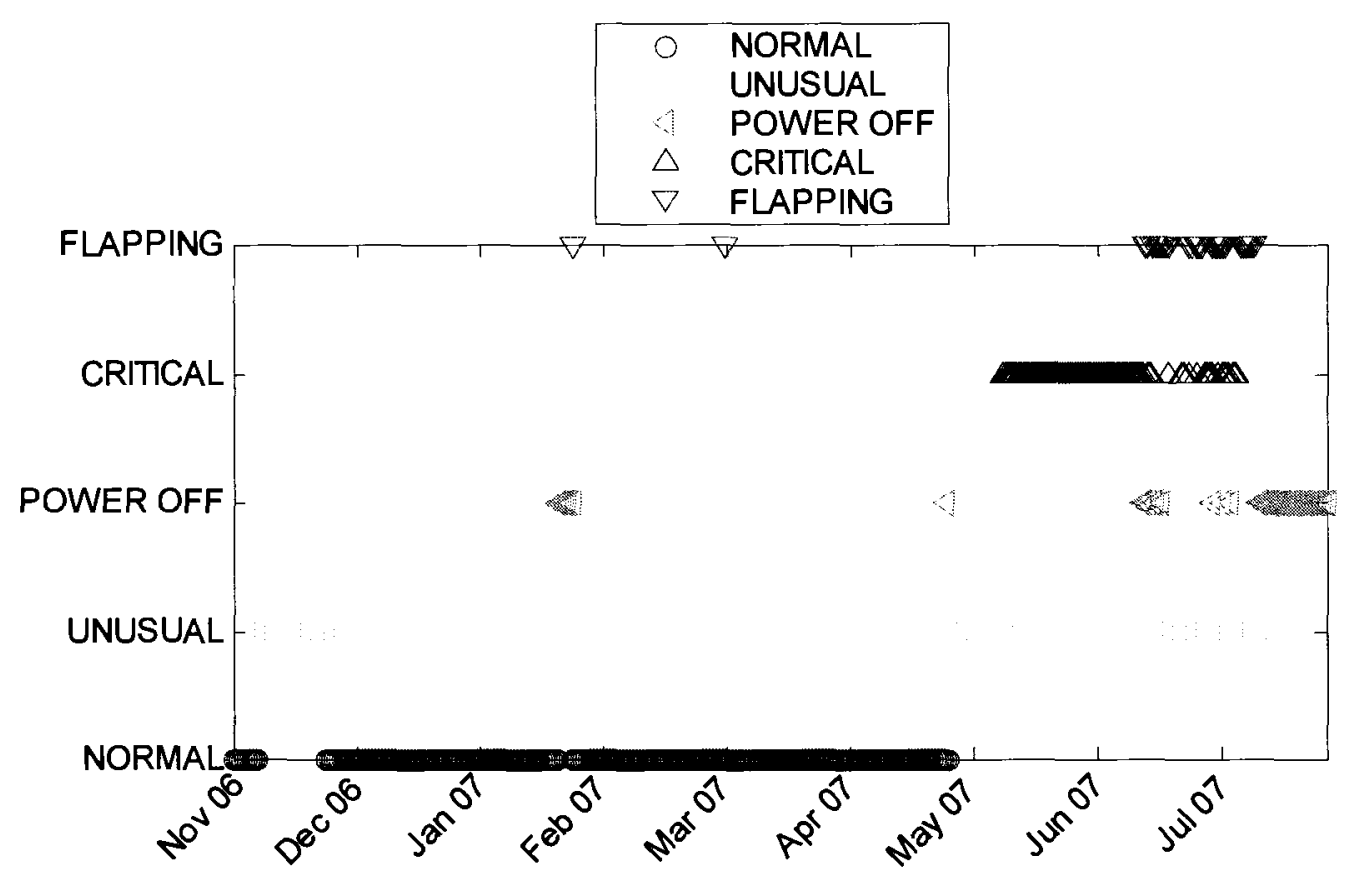

Figure 5.10: Illustrating diagnosis result of Altin station under tight constraint 
Table 5.2: Diagnosis result of Altin station under difference decision constraints

\begin{tabular}{|c|c|c|c|}
\hline & NOMINAL & LOOSE & TIGHT \\
\hline NORMAL & $57.2 \%$ & $57.7 \%$ & $57.7 \%$ \\
\hline UNUSUAL & $13.4 \%$ & $14.9 \%$ & $16.7 \%$ \\
\hline POWER OFF & $8.4 \%$ & $8.3 \%$ & $8.3 \%$ \\
\hline CRITICAL & $15.7 \%$ & $14.7 \%$ & $13.5 \%$ \\
\hline FLAPPING & $5.3 \%$ & $4.3 \%$ & $3.8 \%$ \\
\hline
\end{tabular}

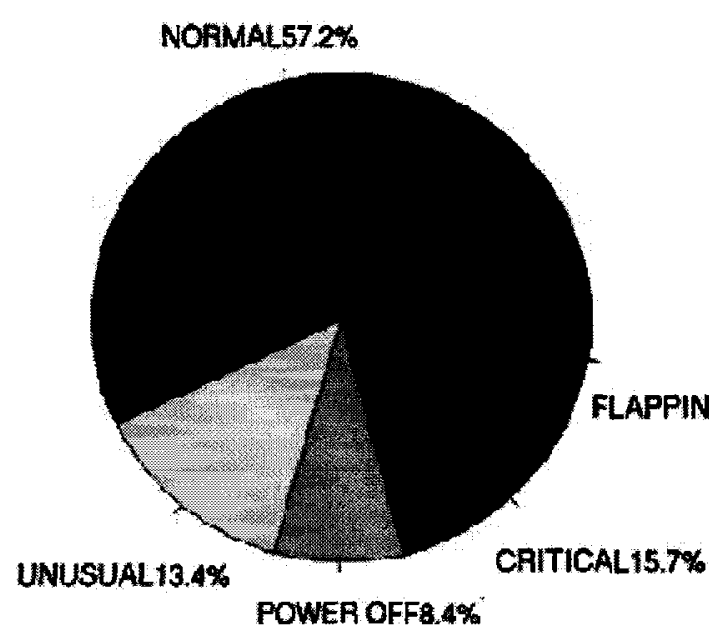

(a) Nominal Constraint

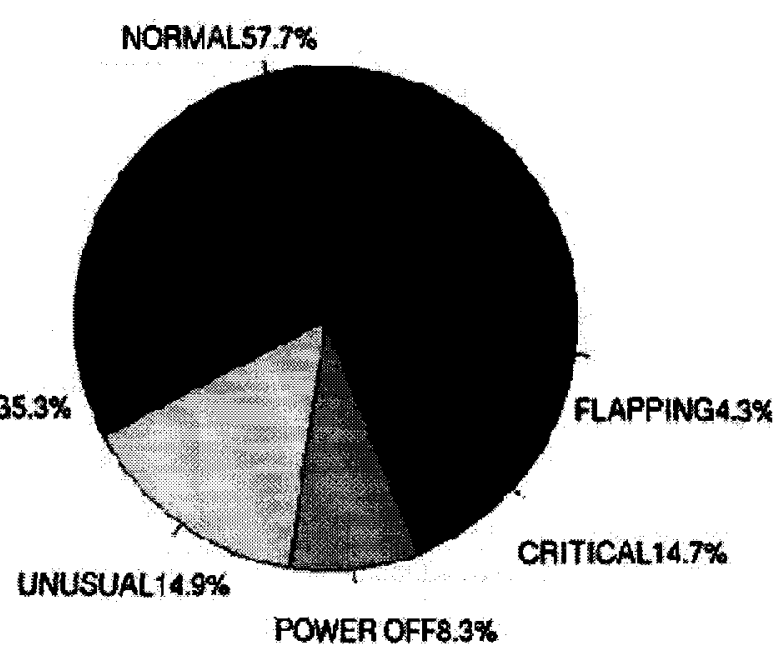

(b) Loose Coustraint

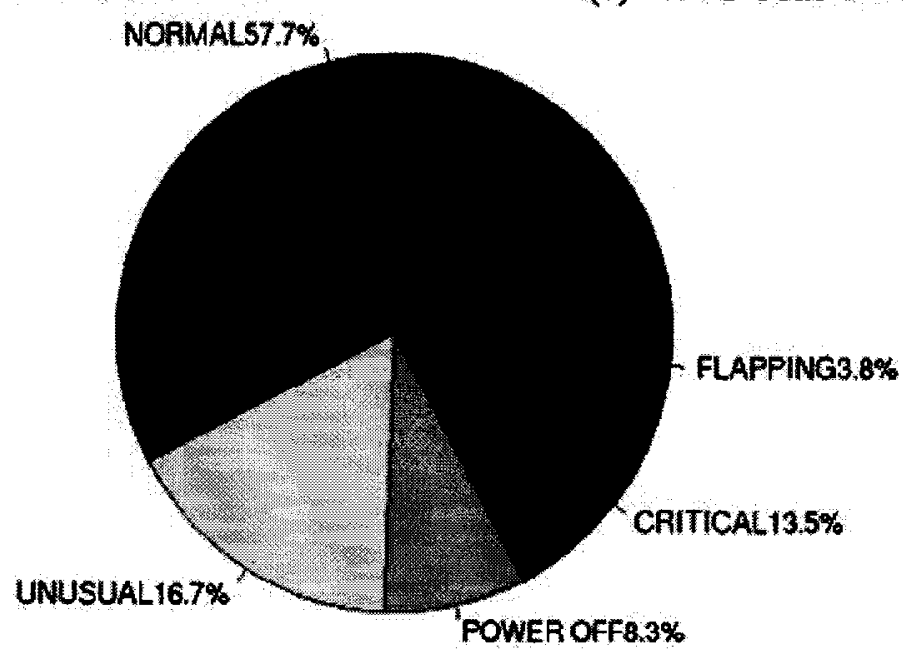

(c) Tight Constraint

Figure 5.11: Illustrating diagnosis result of Altin station using pie chart (a) Nominal Constraint (b) Loose Constraint (c) Tight Constraint 


\subsubsection{Diagnosis Accuracy}

The diagnosis accuracy of the knowledge-based TFD system is evaluated based on two metrics. First, the success ratio is used to represent the diagnosis accuracy of the knowledge-based TFD system. Second, the false alarm ratio is used to represent the percentage of misreported faulty conditions. As shown in the above section, the historical FLS of the Altin station from November 2006 to July 2007 was used to test the overall performance of the knowledge-based TFD system. In this section, the diagnosis results from the same data set were used in order to evaluate the diagnosis accuracy of the knowledge-based TFD system. A total of 2,160 decisions were generated using this data set. As a result, the calculated diagnosis successful ratio and false alarm ratio can more or less represent the actual diagnosis accuracy of the knowledge-based TFD system.

In the knowledge-based TFD system, the operating condition of a GT is classified into one of five categories, which include NORMAL, UNUSUAL, POWER OFF, FLAPPING, and CRITICAL conditions. As described in Chapter 4, the decision boundary of the NORMAL and UNUSUAL conditions are fuzzy; it is hard to tell whether the system misreported on the NORMAL and UNUSUAL conditions. Even experienced satellite operators did not have an accuracy method to decide whether a GT is under the NORMAL or UNUSUAL condition. For this reason, only the diagnosis of the POWER OFF, FLAPPING and, CRITICAL conditions were calculated. Because the FLS patterns of these conditions are obvious, satellite operators have precise methods to identify these three conditions. 
The diagnosis success ratio of diagnosing a type of condition is the ratio of successfully reported faulty conditions over the total number of faulty conditions. We calculated the success ratio of diagnosing POWER OFF, FLAPPING, and CRITICAL conditions using the following logic:

$$
\text { success_ratio }=\frac{\text { number_of _successful_reported_faulty_conditions }}{\text { total_number_of_faulty_conditions }}
$$

where the total number of faulty conditions are all the faulty conditions that occurred; this number was provided by Telesat. The number of successfully reported fault conditions was obtained by analyzing the historical FLS with the help of experienced satellite operators. The diagnosis success ratios for the POWER OFF, FLAPPING, and CRITICAL conditions under different decision constraints are listed in Table 5.3.

Table 5.3: Diagnosis success ratios of knowledge-based TFD system

\begin{tabular}{|c|c|c|c|}
\hline Decision Constraint & Power Off & Flapping & Critical \\
\hline Nominal Constraint & 0.971 & 0.811 & 0.867 \\
\hline Loose Constraint & 0.971 & 0.811 & 0.867 \\
\hline Tight Constraint & 0.971 & 0.811 & 0.867 \\
\hline
\end{tabular}

As shown in Table 5.3, the diagnosis accuracy of the POWER OFF, FLAPPING, and CRITICAL conditions exceeded $80 \%$ under all decision constraints. The diagnosis success ratio of the POWER OFF condition was 97.1\% under all decision constraints. The design is very successful, because this successful ratio is very close to the successful ratio of satellite operators. The diagnosis accuracy of the FLAPPING condition was $81.1 \%$ under all decision constraints. This successful ratio is high compared to the decisions of satellite operators. When diagnosing a FLAPPING scenario, satellite 
operators need to contact the local customers to confirm the event. It is a great success that the knowledge-based TFD system can correctly diagnosis the FLAPPING conditions over $80 \%$ of the time. The success ratio for diagnosing the CRITICAL condition was 86.7\%. Diagnosing the CRITICAL condition also needs confirmation from customers; therefore, this successful ratio is acceptable when only using historical FLS. Because the effectiveness of the rule sets decided the number of successful diagnosed conditions, increased decision constraints did not improve the success ratio of the knowledge-based TFD system. However, increasing the decision constraints can eliminate some misreported conditions.

The false alarm ratio of the knowledge-based TFD system can be calculated using the following logic:

$$
\text { false_alarm_ratio }=\frac{\text { number_of } \text { of }_{-} \text {misreported_faulty_consditions }}{\text { total_number_of_reported_faulty_conditions }}
$$

where the number of misreported faulty conditions are the number of faulty conditions that were reported incorrectly by the TFD system. The numbers of the misreported faulty conditions are obtained by doing a sanity check on test results with help from experienced satellite operators. The false alarm ratios of the knowledge-based TFD system with different decision constraints are shown in Table 5.4.

Table 5.4: Diagnosis false alarm ratios of knowledge-based TFD system

\begin{tabular}{|c|c|c|c|}
\hline Decision Constraint & Power Off & Flapping & Critical \\
\hline Nominal Constraint & 0.073 & 0.348 & 0.217 \\
\hline Loose Constraint & 0.063 & 0.198 & 0.167 \\
\hline Tight Constraint & 0.063 & 0.098 & 0.091 \\
\hline
\end{tabular}


As shown in Table 5.4, the false alarm ratio for diagnosing all three conditions was below $10 \%$ under tight constraint. The false alarm ratio of diagnosing the POWER OFF condition dropped to $6.3 \%$ under loose and tight constraint; these results were very satisfactory. For the FLAPPING condition, the false alarm ratio was $34.8 \%$ under nominal constraint. It dropped to $9.8 \%$ as the decision constraint increased to tight. Because when the constraint increased the number of misreported faulty conditions decreased, the false alarm ratio decreased. The false alarm ratio for diagnosing the CRITICAL condition also dropped from $21.7 \%$ to $9.1 \%$ when the decision constraint changed from nominal to tight.

Overall, diagnosing the accuracy of POWER OFF, FLAPPING and CRITICAL conditions was satisfactory. The success ratio of diagnosing the POWER OFF condition was the highest among all conditions. The success ratio of diagnosing the FLAPPING and CRITICAL conditions exceeded $80 \%$. The false alarm ratio for diagnosing the FLAPPING and CRITICAL conditions were high under nominal constraint, but the ratio dropped to about $10 \%$ as the decision constraint changed from nominal to tight. Tight constraint is recommended because when operating under tight constraint, we have the lowest false alarm ratio for diagnosing all three conditions. 


\subsubsection{Performance Comparison of Single Mode and Neighbouring Mode}

The neighbouring mode is designed to collaborate with the FLS measured in the neighbouring GTs in order to diagnose the operating conditions of GTs. In this section, we evaluated the advantages of the neighbouring mode by comparing the diagnosis results of the neighbouring mode to the diagnosis results of the single mode.

The Hazelton station was selected to be the targeted GT, and the Terrace station was selected to be the neighbouring GT. The historical FLS of both GTs from August 1, 2007 to December 31, 2007 were selected as the diagnostic source. The 3 hour average FLS of the Hazelton station and the Terrace station are plotted in Figure 5.12 and Figure 5.13 respectively. At the beginning, the FLS of both stations showed regular fluctuation. According to past experience, the expected FLS of the Hazelton station is set to $8 \mathrm{~dB}$, and the expected FLS of the Terrace station is set to $10 \mathrm{~dB}$. The FLS of the Hazelton station deteriorated gradually starting from October until the end of November. In early December, the FLS of the Hazelton station recovered slightly but still fluctuated below the expected FLS. The FLS patterns of the Terrace station shared similar trends with the Hazelton station. Different from FLS patterns of the Hazelton station, the FLS of the Terrace station started to drop gradually in mid October and experienced a harsh period at the end of October. When diagnosing the Hazelton station with FLS from itself, the system will consider the deteriorating performance as an UNUSUAL condition. However, since its neighbour also experienced a similar deteriorating performance, the system assumed the deteriorating performance is caused by a common change in the surrounding environment, and the GT will be able to recover. Therefore, when 
diagnosing the Hazelton station using the neighbouring mode, some UNUSUAL conditions diagnosed in the single mode will be reported as NORMAL conditions.

The operating condition of the Hazelton station is diagnosed in both the single mode and neighbouring mode with tight constraint. Figure 5.14 compares the diagnosis results using a pie chart. As shown in the figure, using the neighbouring mode reduced $22 \%$ of the UNUSUAL conditions, and the percentage of NORMAL conditions increased correspondingly. Figure 5.15 illustrates the change of NORMAL and UNUSUAL conditions when diagnosing with different modes. Under the single mode, the operating conditions of the Hazelton station changed from NORMAL to UNUSUAL at the end of October 2007 and stayed Unusual ever since. Thanks to the collaboration of neighbouring GTs, the knowledge-based TFD system only reported the UNUSUAL condition of the Hazelton station for three short periods in early November, mid December, and late December. Therefore, the neighbouring mode improved the accuracy of diagnosing the NORMAL condition. In the Hazelton example, the percentage of NORMAL conditions increased by $22 \%$ in the neighbouring mode. If there is neighbouring GT available, we recommend using the neighbouring mode. 


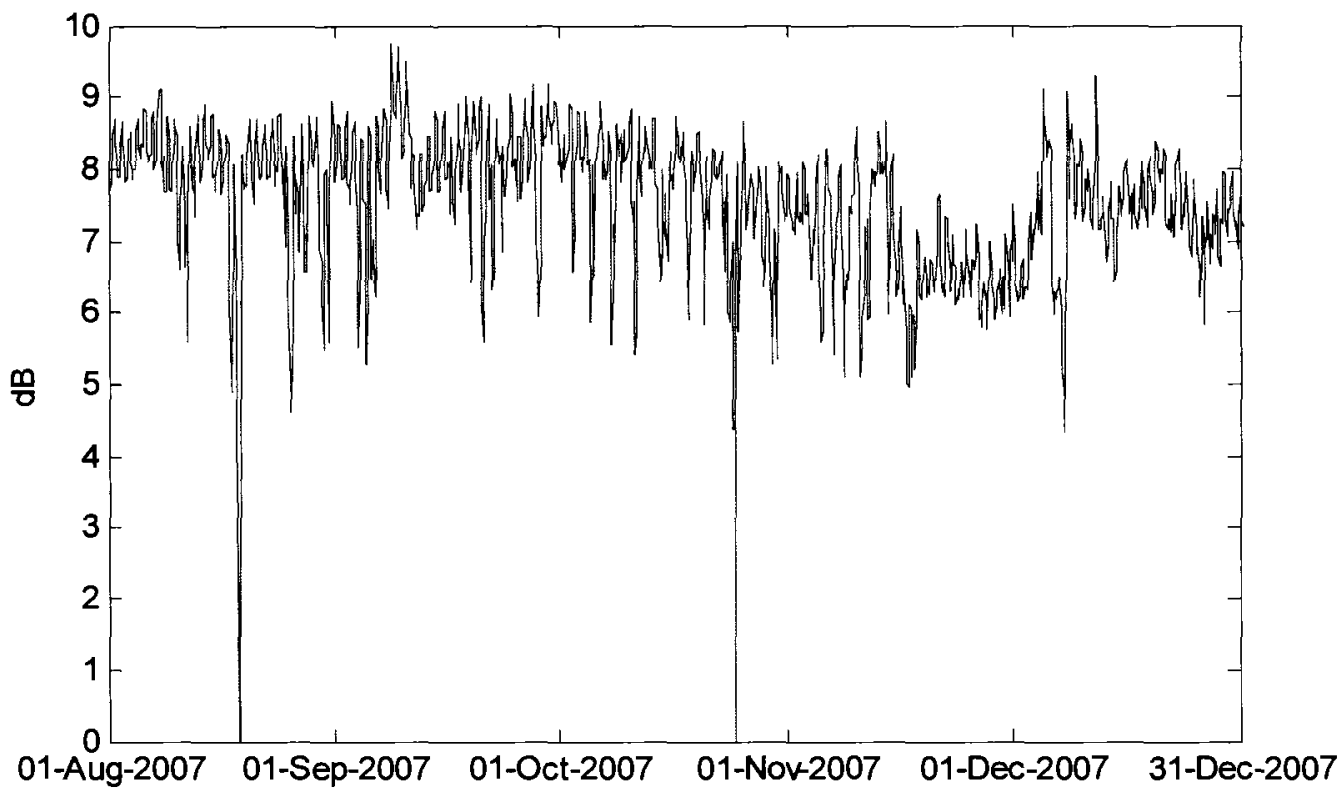

Figure 5.12: FLS of Hazelton station from August 2007 to December 2007 (3 hour average)

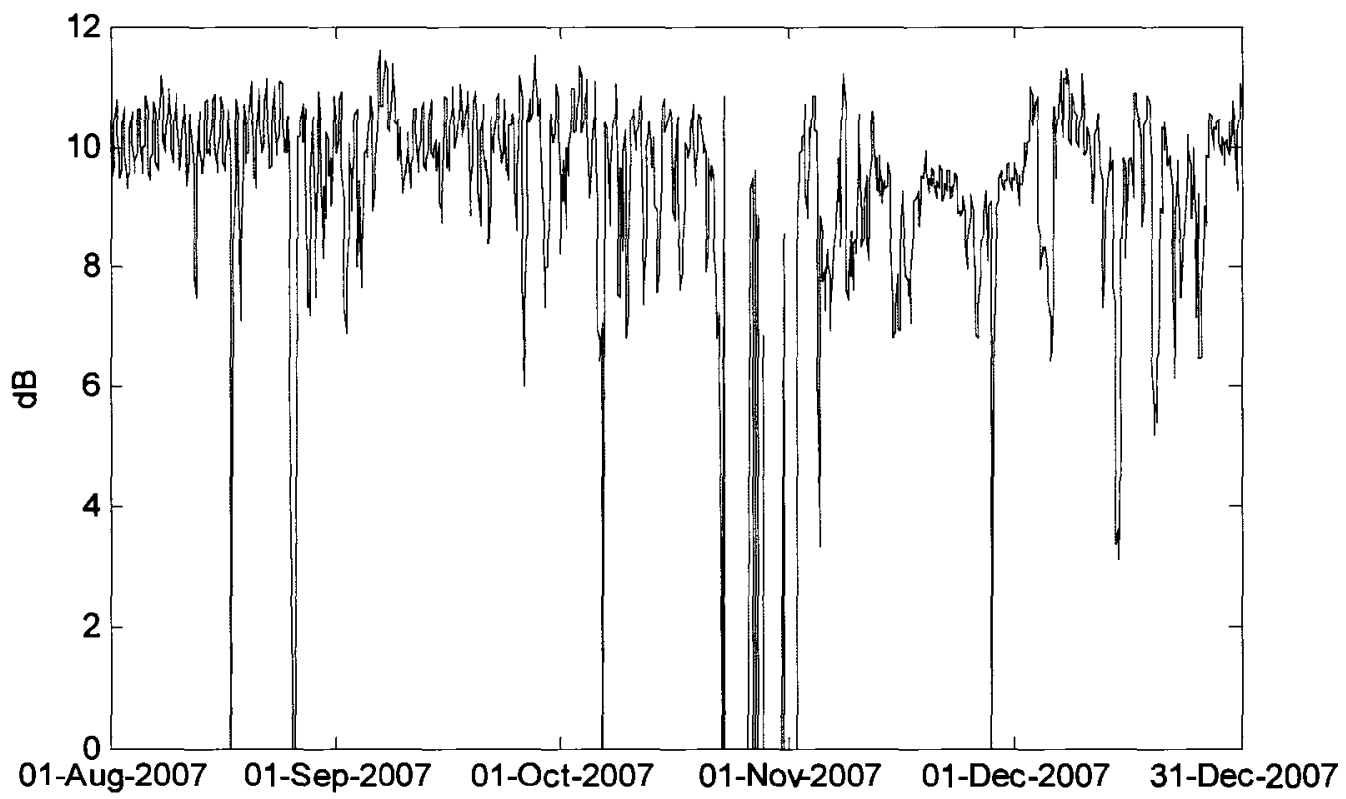

Figure 5.13: FLS of Terrace station from August 2007 to December2007 (3 hour average) 


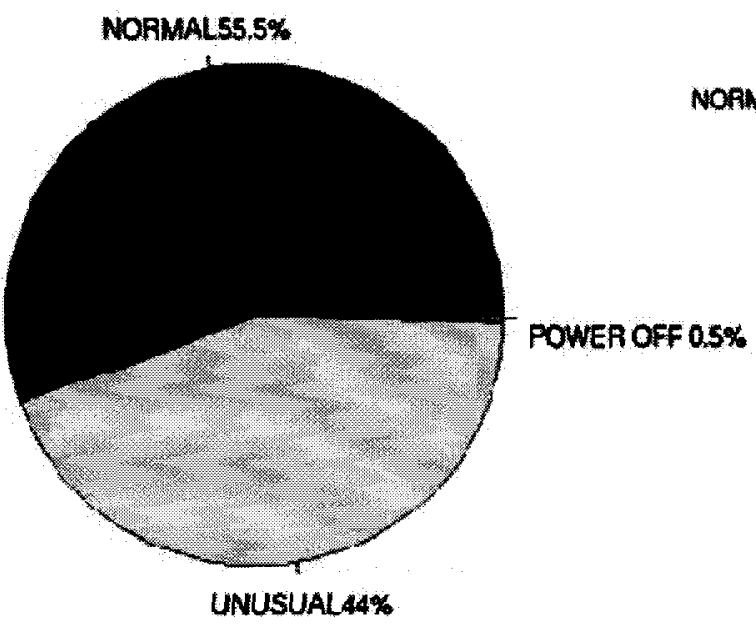

(a) Single Mode

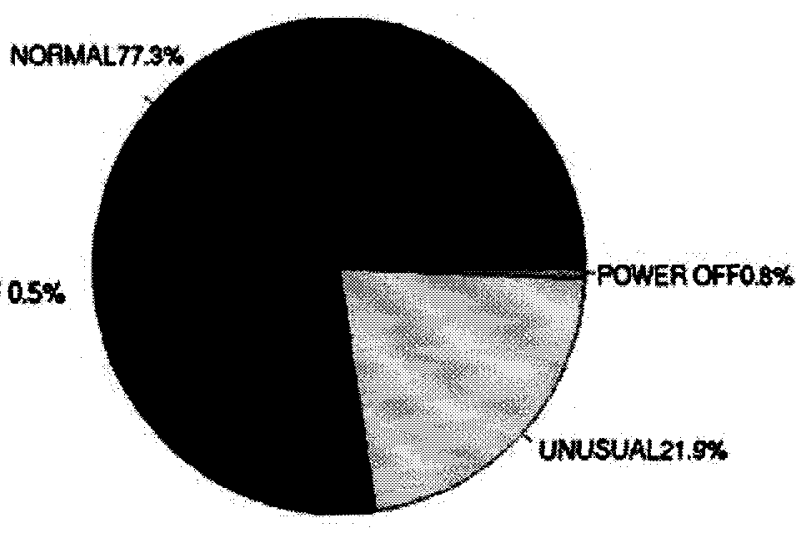

(b) Neighbouring Mode

Figure 5.14: Comparing diagnosis result of Hazelton station under tight constraint (a) single mode (b) neighboring mode

(a) Diagnosis Result under Single Mode

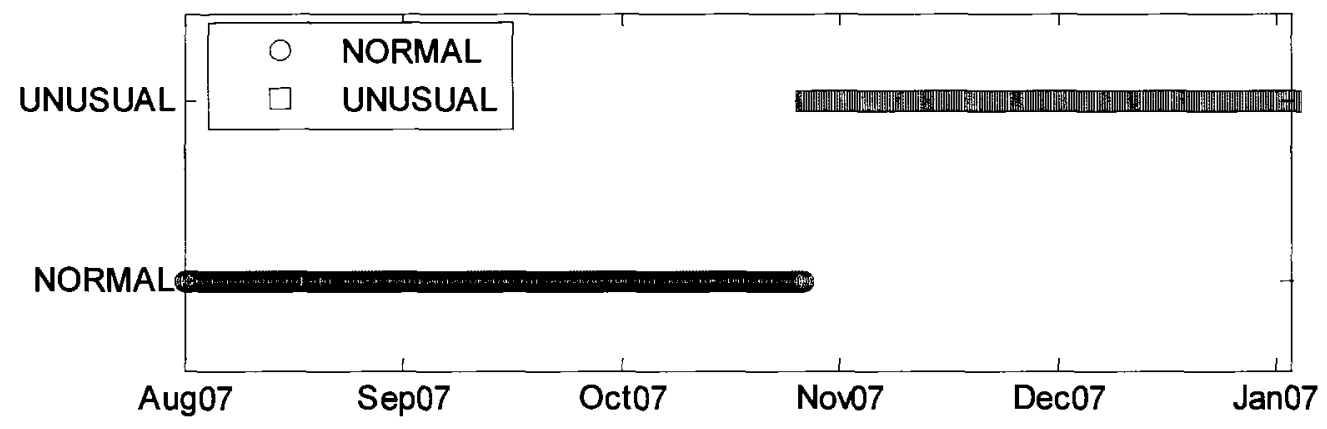

(b) Diagnosis Result under Neighbouring Mode

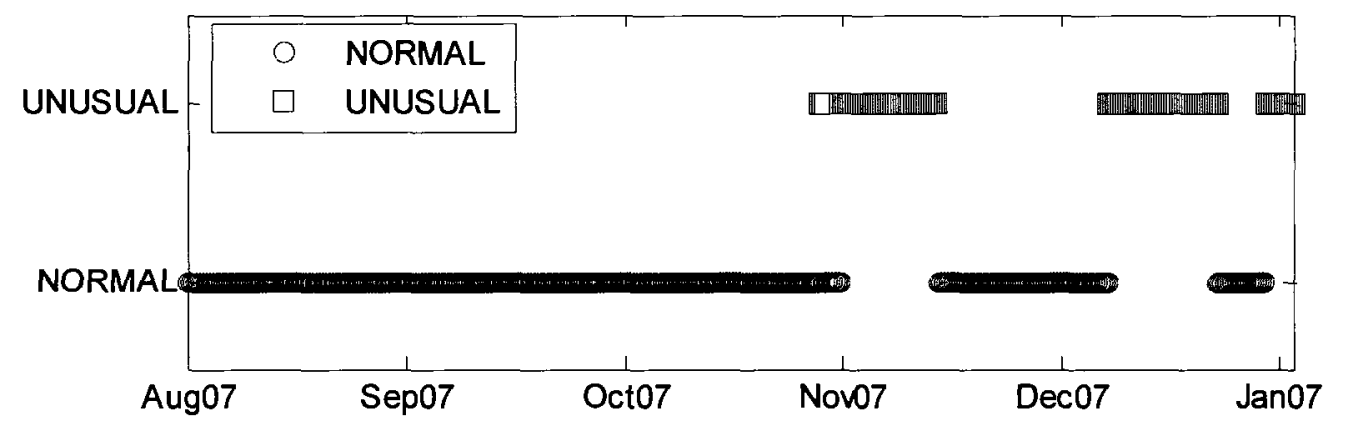

Figure 5.15: Illustrating diagnosis result of Hazelton station with tight constraint 


\section{Chapter 6}

\section{Conclusions and Suggestions for Future Work}

\subsection{Conclusion}

In this thesis, a fuzzy-logic based congestion adaptation system and a knowledge-based TFD system were developed for a broadband satellite network. The designed and implemented intelligent system can be deployed at the satellite hub, and the ground terminal of the broadband satellite network can overcome the inherent impacts of the dynamic weather conditions.

The congestion control system uses the rain/snow-fall rate to tune system parameters, which is a new concept in congestion control of territorial IP networks. A unique set of membership functions was designed in order to properly represent the rain/snow-fall rate. A knowledge base is also constructed using the knowledge of experienced network engineers. The test results revealed that the implementation of a congestion adaptation system was practical and effective on the broadband satellite system. The congestion adaptation system responds to different rain/snow-fall rates, and it limits the traffic flow into the satellite network. On the other hand, the priority traffic streams are prioritized 
when the channel capacity decreases. The trial on Telesat's broadband satellite network test-bed was successful and proved the operability of the system.

The knowledge-based system is the first of its kind to use expert knowledge in diagnosing the operating conditions of satellite GTs. A sliding window algorithm was implemented in order to extract the trending information of a GT based on historical FLS. The outputs are generated by a hybrid system, which contains a statistical method and a knowledge-based method. This approach introduced a new concept to the literature, and the proposed system was fully tested using the historical data provided by Telesat.

The knowledge-based TFD system has a very high diagnosis accuracy when diagnosing the POWER OFF, FLAPPING, and CRITICAL conditions. The system can correctly diagnose the POWER OFF condition $97.1 \%$ of the time. The success ratio for diagnosing FLAPPING conditions reached $81.1 \%$, and the success ratio of the CRITICAL condition was $86.7 \%$. The system successfully reduced the false alarm ratio of diagnosing all three conditions by using different decision constraints. The false alarm ratio used for diagnosing the POWER OFF condition was minimized to $6.3 \%$ under tight constraint, and the false alarm ratio for diagnosing the FLAPPING condition was reduced to $9.8 \%$ under tight constraint. The false alarm ratio for diagnosing the CRITICAL condition was limited to $9.1 \%$ under tight constraint. Overall, the design of the knowledge-based system was successful. The system properly represented expert knowledge and made accurate diagnoses.

In addition, the design of the neighbouring mode is also verified using the historical RF data, which was provided by Telesat. Test results proved that the neighbouring mode 
can reduce the number of misreported UNUSUAL conditions by $22 \%$. The effectiveness of the neighbouring mode was also verified and confirmed by the satellite service provider.

In the future, the knowledge-based TFD system will be deployed in the fields in order to diagnose GTs in real-time. The rule sets could be finalized and completed when more test results from daily satellite operations are available. 


\subsection{Suggestions for Future Work}

The performance of the proposed congestion adaptation system is very sensitive to network configurations and parameter settings. As a result, it is necessary to tune the congestion adaptation system for different network topologies and configurations in order to achieve the best possible performance. In the future, field testing will be scheduled in order to evaluate the performance of the software. Work has to be done to automate the configuration process. With the help of testing results from the field test, tuning the system will be able to be accomplished efficiently.

For a knowledge-based TFD system, the knowledge base is created according to a comprehensive analysis of historical FLS measurements. The knowledge base classified the operating conditions of GTs into five distinct categories. For events that do not belong to one of these five categories, the TFD system will classify them into the closest categories base on their FLS trends. As a result, the system may misreport some unexpected operating conditions. Developing a subsystem to handle unexpected events will help satellite operators learn from unexpected operating conditions. The knowledgebased TFD system is designed to be upgradeable without major changes to the system. On the other hand, new rules need to be added to the knowledge base in order to improve the diagnosis accuracy of the FLAPPING and CRITICAL conditions. It is also prudent to study the FLS of each GT and customize the rule sets for each GT. 


\section{Appendix A: Anik-F2 Specification and Coverage Map}

\section{PAYLOAD}

\begin{tabular}{|l|l|}
\hline C-band & 24 Active, 30-w TWTAs \\
\hline Ku-band & 32 active (8 spare) 127-w TWTAs \\
\hline Ka-band & 38 Active (12 spare) 90-w TWTAs \\
\hline
\end{tabular}

\section{POWER}

\begin{tabular}{|l|l|}
\hline Solar: Beginning of life, End of life & $16 \mathrm{kw}, 15 \mathrm{kw}$ \\
\hline Batteries & 58 cell NiH, 328 Ahr \\
\hline
\end{tabular}

\section{DIMENSIONS}

\begin{tabular}{|l|l|}
\hline In Orbit & $\begin{array}{l}\text { L: solar arrays: } 47.9 \mathrm{~m}(157 \mathrm{ft}) \\
\text { W: antennas: } 8.2 \mathrm{~m}(27 \mathrm{ft})\end{array}$ \\
\hline Stowed & H: $7.3 \mathrm{~m}(24 \mathrm{t})$ \\
& W: $3.8 \mathrm{~m} \times 3.4 \mathrm{~m}(12.5 \mathrm{ft} \times 11.2 \mathrm{ft})$ \\
\hline $\begin{array}{r}\text { Mass: Launch } \\
\text { In orbit }\end{array}$ & $\begin{array}{l}5950 \mathrm{~kg}(13118 \mathrm{lbs}) \\
3805 \mathrm{~kg}(8390 \mathrm{lbs})\end{array}$ \\
\hline
\end{tabular}

\section{ANTENNAS}

Nadir two 85" dual shaped girded reflectors - (one C-band and one Ku-band)

Nadir: one Ka-band beacon transmit array

Four 55" transmit reflectors (Ka-band)

Four 36" receive reflectors (Ka-band)

Two 20" track reflectors (Ku-band) 


\section{ANIK F2 Ka-Band Edge of Coverage Patterns}

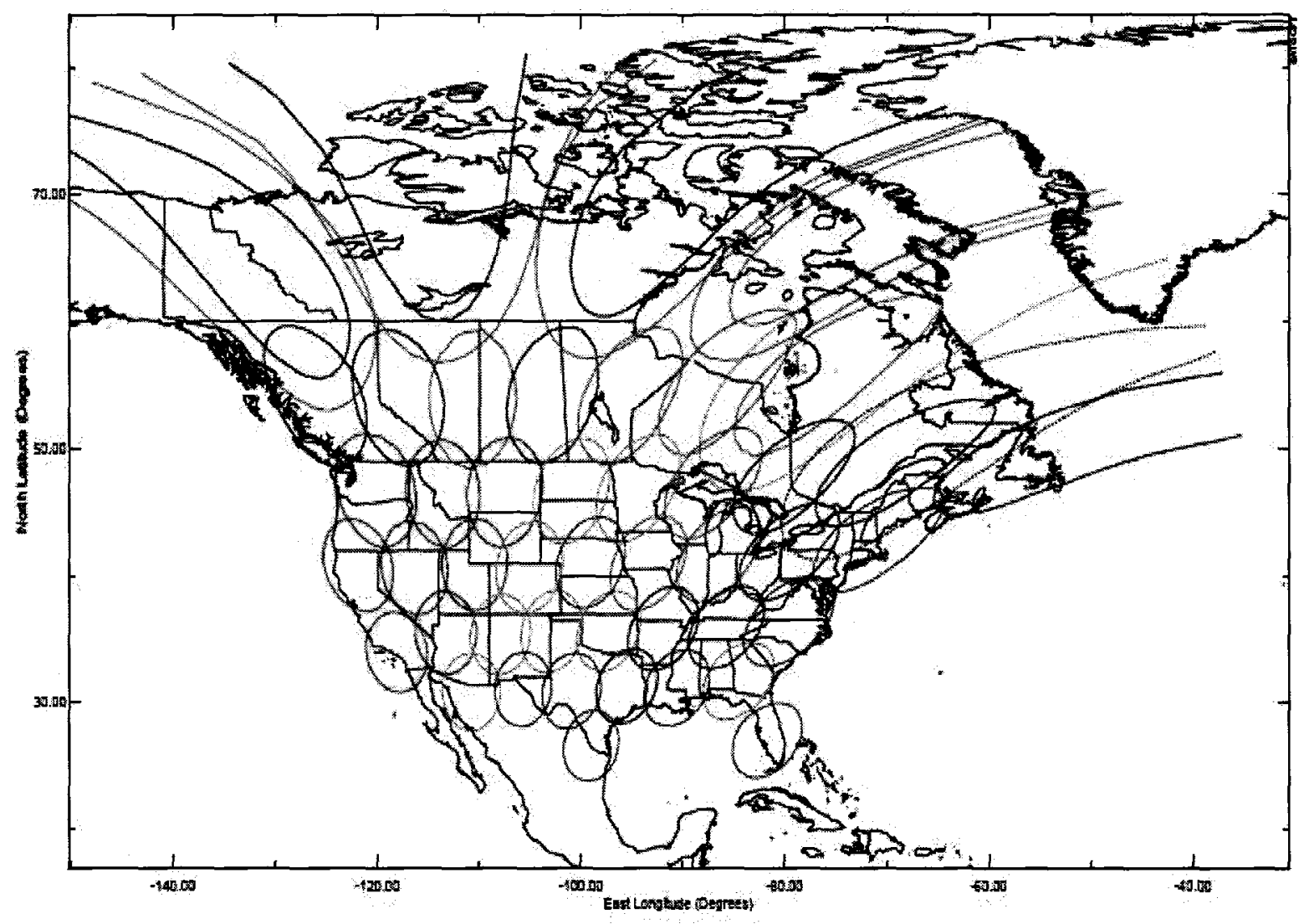




\section{Appendix B: Satellite Terminal Locations}

\begin{tabular}{|c|c|c|c|}
\hline Location & Site Code & Latitude & Longitude \\
\hline Atlin & ALN & $59.58 \mathrm{~N}$ & $133.72 \mathrm{~W}$ \\
\hline Yellow knife & YLK & $62.42 \mathrm{~N}$ & $114.42 \mathrm{~W}$ \\
\hline Hazelton & HZN & $55.25 \mathrm{~N}$ & $127.67 \mathrm{~W}$ \\
\hline Terrace & TRC & $54.50 \mathrm{~N}$ & $128.52 \mathrm{~W}$ \\
\hline Dease Lake & & $58.25 \mathrm{~N}$ & $130.10 \mathrm{~W}$ \\
\hline Arviat & & $61.07 \mathrm{~N}$ & $94.04 \mathrm{~W}$ \\
\hline Iqaluit & & $63.45 \mathrm{~N}$ & $68.30 \mathrm{~W}$ \\
\hline Pond Inlet & & $72.42 \mathrm{~N}$ & $77.59 \mathrm{~W}$ \\
\hline Vancouver & VAN & $49.27 \mathrm{~N}$ & $123.09 \mathrm{~W}$ \\
\hline Ottawa & HQD & $45.43 \mathrm{~N}$ & $75.60 \mathrm{~W}$ \\
\hline
\end{tabular}

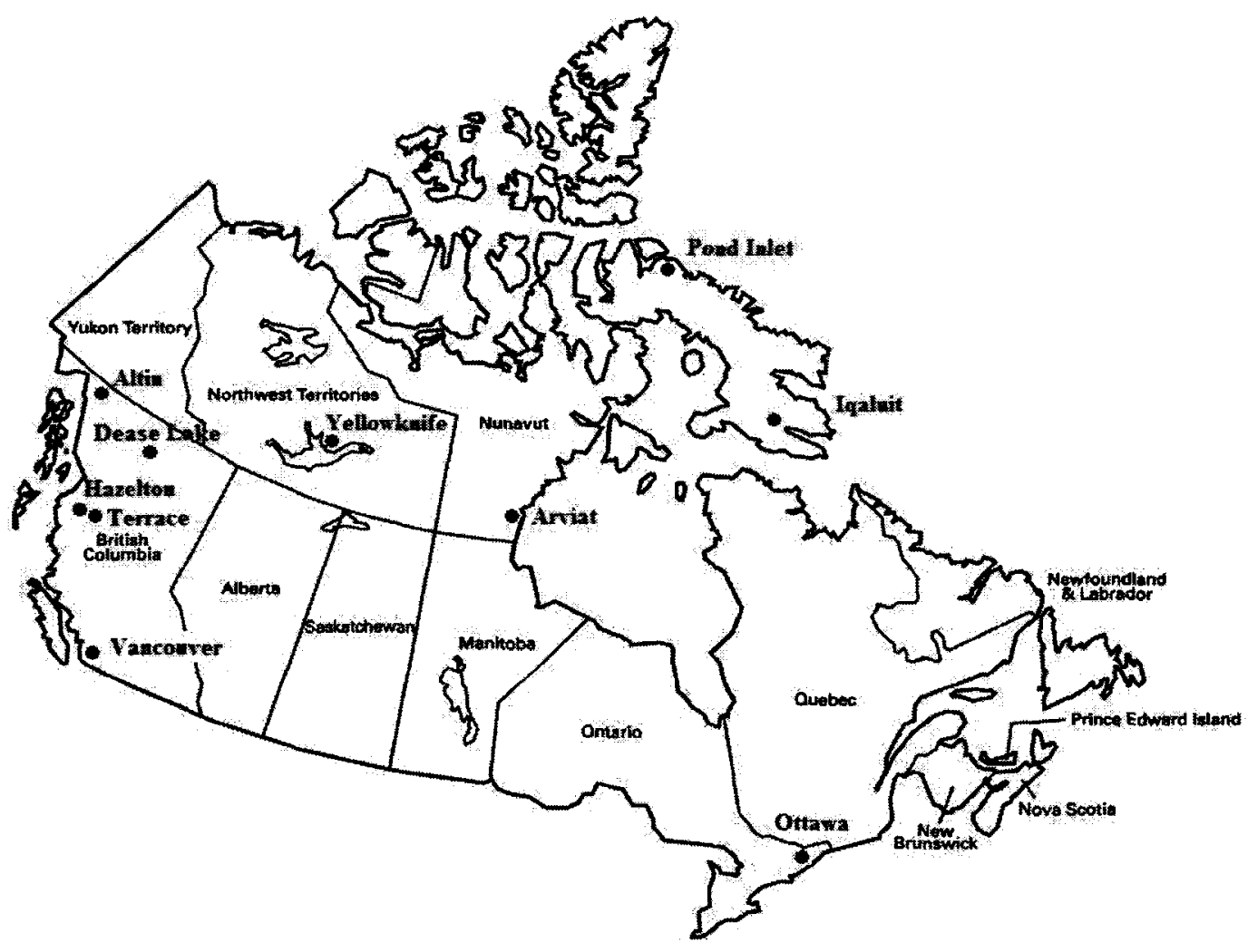




\section{Appendix C: Fuzzy Logic Theory}

\section{C.1 Fuzzy logic theory}

Fuzzy Logic was first developed by L. A. Zadeh in [41]; its purpose is to represent the various types of "approximate" knowledge, which cannot be represented by numerical methods. Fuzzy logic is an extension of the two-state logic, and it provides a better platform for handling approximate knowledge [42].

Fuzzy logic is based on fuzzy sets, and a fuzzy set is represented by a Membership Function (MF). A fuzzy set is a set without a clearly defined boundary, and it contains partial elements from the universe of discourse, where the universe of discourse represents every possible value of a given discussion. For example, the universe of discourse of temperature may contain all the possible temperatures from absolute zero degree to infinite. A fuzzy set Cold contains only partial elements of the universe of discourse of temperature. A MF is a curve that defines how each point in a fuzzy set is mapped to a membership value from 0 to 1 . The membership value assigned to an element is also known as a degree of truth. The degree of truth describes how each point in the universe of discourse is mapped into different fuzzy sets. If $X$ is the universe of 
discourse, and elements in $X$ are denoted by $x$, then a fuzzy set $A$ in $X$, which represents part of $X$ is defined as the set of ordered pairs as follow:

$$
A=\left\{x, \mu_{A}(x) \mid x \in X\right\}
$$

where $\mu_{A}(x)$ is a MF of $x$ in $A$. The MF maps each element of $\mathrm{X}$ to a MF value from 0 to 1. MF could be either piecewise linear or quadratic. Figure 1 shows the most frequently used MFs where the $\mathrm{x}$-axis is the range of universe of discourse and the $\mathrm{y}$-axis represents the degree of truth of all the elements in the fuzzy sets.

In the following example, $\mathrm{S}$ functions and $\mathrm{Z}$ functions were used to map the temperature of water into three fuzzy sets which are COLD, WARM, and HOT. Because water freezes at 0 degree and boils at 100 degrees under standard atmospheric pressure, the universe of discourse for the temperature of water is from 0 degree to 100 degrees. Figure 2 below shows how the temperature of water is represented by fuzzy set COLD, WARM, and HOT. FuzZy set COLD uses the $Z$ function, because as the temperature of water gets closer to 0 degree, it is more likely to indicate that it is cold. As shown in figure 1 , the $\mathrm{Z}$ function assigns a membership value 1 to all the points below threshold $a$ and gradually decreases membership value to 0 from threshold $a$ to $c$. This can properly represent the fuzzy set COLD. The S function was used to represent the fuzzy set HOT for the same reason. Warm is an ambiguous definition. Water temperature at 20 degrees can be said to be warm. On the other hand, water temperature at 30 degrees could also be considered warm. The $\pi$ function was used in order to represent the fuzzy set WARM, because the $\pi$ function assigns a membership value 1 to a point and gradually decreases 
the membership value as the value of universe of discourse increases or decreases. The Triangle function has the same property as the $\pi$ function, but has linear shape.

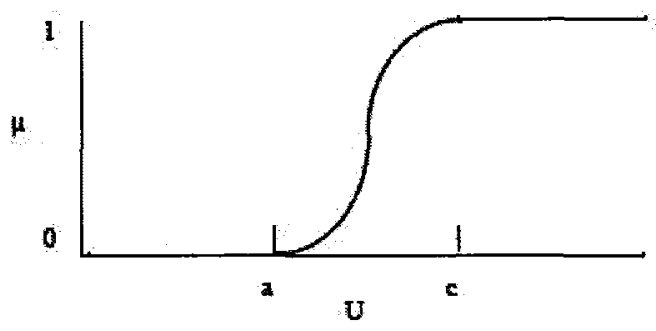

(a) S finction

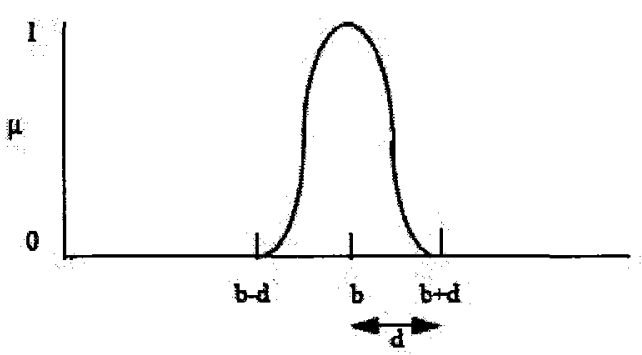

U

(d) I function

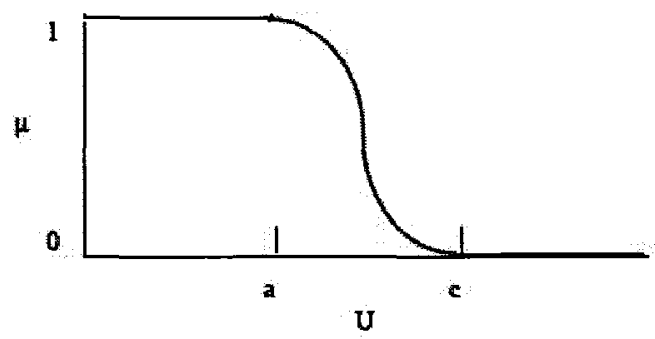

(b) $Z$ function

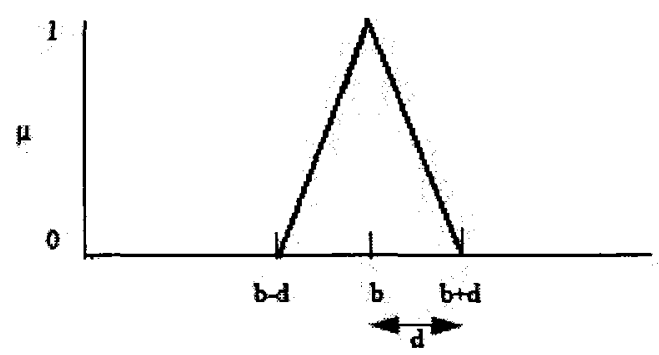

$\mathrm{U}$

(c) Triangle function

Figure 1: Frequently used membership functions [43]

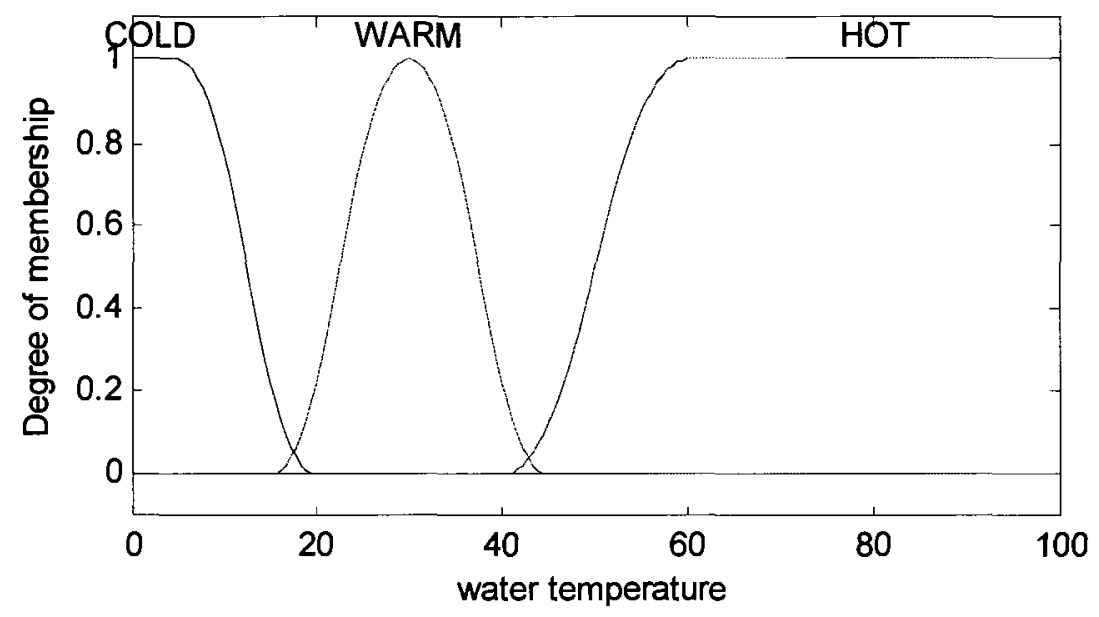

Figure 2: Mapping water temperature into fuzzy sets

When a real value is mapped to a fuzzy set, a linguistic variable is generated. The linguistic variable consists of the name of the fuzzy set and the degree of truth of the 
fuzzy set. As a result, an element in the universe of discourse can belong to a fuzzy set with some degree of truth, and it can also belong to another set with a different degree of truth. An example demonstrating the linguistic variables is mapping water temperature into fuzzy sets. The water temperature is described by three fuzzy sets, namely: COLD, WARM, and HOT. Their membership functions are shown in Figure 3. In the figure, the $\mathrm{x}$-axis displays the range of temperatures from 0 degree to 100 degrees, and the $\mathrm{y}$-axis represents the degree of truth of each element in all the fuzzy sets. If the water temperature is 20 degrees, then the water temperature will be mapped into two linguistic variables COLD and WARM with different degrees of truth. 20 degrees belongs to the fuzzy set COLD with a degree of truth equal to 0.75 . It also belongs to the fuzzy set WARM with degree of truth equivalent to 0.25 .

The Boolean logic operators: AND, OR, and NOT are adopted as Zadeh operators in his original paper on fuzzy logic. Instead of dealing with a crisp value of 0 or 1 , the Zadeh operator deals with truth values between 0 and 1 . The operation of Zadeh operator is shown in Table 1 below. Since each element in the fuzzy set is assigned a degree of truth from 0 to 1 , the Zadeh operators operate based on the degree of truth of the elements. Given two fuzzy sets $\mathrm{x}$ and $\mathrm{y}$ and let $\operatorname{truth}(\mathrm{x})$ and truth(y) denote the degree of truth for all the elements in $\mathrm{x}$ and $\mathrm{y}$, the AND operator returns to the minimum value of $\operatorname{truth}(\mathrm{x})$ and $\operatorname{truth}(\mathrm{y})$. The OR operator returns the maximum value of $\operatorname{truth}(\mathrm{x})$ and $\operatorname{truth}(\mathrm{y})$. The NOT operator returns the value of one minus the degree of truth of an element in a given fuzzy set. In Figure 3, the fuzzy set of WARM is derived from the fuzzy set COLD and HOT using the NOT and OR operator. The fuzzy logic operation can be expressed as: 
Table 1: Functionality of Zadeh operator

$$
\begin{aligned}
& \text { NOT } \mathrm{x}=(1-\operatorname{truth}(\mathrm{x})) \\
& \mathrm{x} \text { AND } \mathrm{y}=\operatorname{minim} \text { m }(\operatorname{truth}(\mathrm{x}), \operatorname{truth}(\mathrm{y})) \\
& \mathrm{x} \text { OR } \mathrm{y}=\operatorname{maxim} \text { un }(\operatorname{truth}(\mathrm{x}), \operatorname{tuth}(\mathrm{y}))
\end{aligned}
$$

Linguistic Vahe: HOT (), COLD (+), WARM (not[hot or cold]) (*)

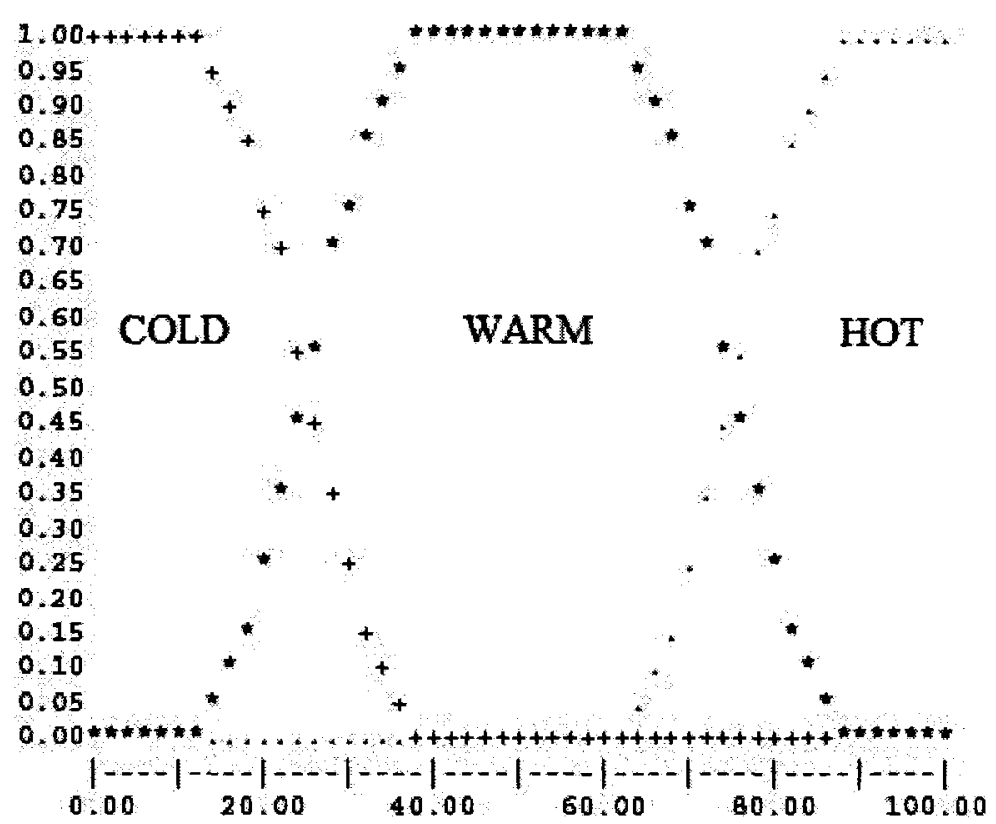

Figure 3: Linguistic variables of temperature of water [43]

The degrees of membership function are often confused with probabilities. However, they are conceptually distinct. The fuzzy degree represents membership in vaguely defined sets. On the other hand, the probability defines the likelihood of some event or condition. 
In building a knowledge-based expert system, fuzzy logic uses IF/THEN rules to represent a human expertise and thinking patterns. A single IF-THEN rule assumes the following format:

\section{If $\mathrm{X}$ is $\mathrm{A}$ then $\mathrm{Y}$ is $\mathrm{B}$}

where $\mathrm{A}$ and $\mathrm{B}$ are linguistic variables that are defined by fuzzy sets in the range of $X$ and $Y$. The first half "If X is A" is called the antecedent, and the second half "Y is B" is called the consequent. The degree of truth of the consequent is the same as the degree of truth of the antecedent. The rules in the knowledge base can include multiple consequents as illustrated in Equation 4:

\section{If $\mathrm{X}$ is $\mathrm{A}$ then $\mathrm{Y}$ is $\mathrm{B}$ and $\mathrm{z}$ is $\mathrm{C}$}

where all consequents have the same degree of truth as the linguistic variable in the antecedent. Rules can also have multiple antecedents which have the format in Equation 5 below:

\section{If $X$ is $A$ and $Y$ is $B$ then $Z$ is $C$}

Linguistic variable $\mathrm{A}$ and $\mathrm{B}$ in the antecedent may have different degrees of truth. The degree of truth of $\mathrm{C}$ in Equation 5 is decided using the Equation 6:

$$
\operatorname{truth}(\mathrm{C})=\text { MIN }(\operatorname{truth}(\mathrm{A}), \operatorname{truth}(\mathrm{B}))
$$

where truth(A) denotes the degree of truth of linguistic variable A. Therefore, the degree of truth for the consequent is the smallest degree of truth of the linguistic variables in the antecedent.

In the fuzzy inference process, multiple rules can be executed at the same time. The consequents from each rule that are being executed are combined into a union of fuzzy 
sets using the method shown in Figure 4 below. The union of fuzzy sets aggregates the consequent from each rule and uses the maximum degree of truth as the overall degree of truth.

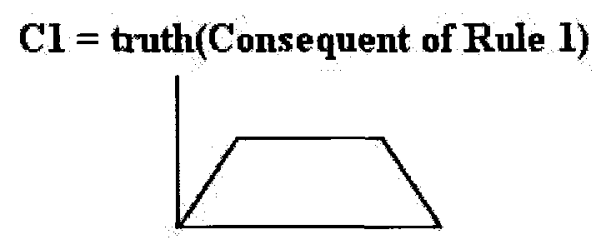

C2 = truth(Consequent of Rule 2)

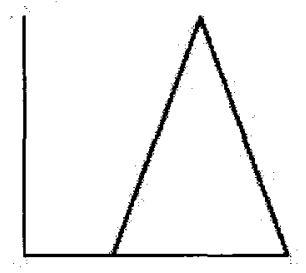

$$
\mathrm{C}=\text { truth (union of furzy sets) }
$$

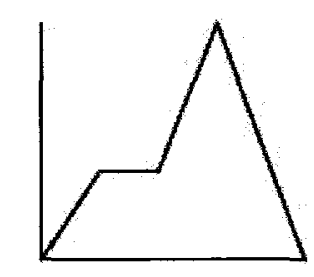

$$
\mathrm{C}=\operatorname{MAX}(\mathrm{C1}, \mathrm{C} 2)
$$

Figure 4: Compositional rules for union of fuzzy sets [43]

In order to obtain a meaningful output of the fuzzy system, the union of the fuzzy sets needs to be defuzzified. The Center of gravity method is widely adopted as a defuzzification method for fuzzy systems. It can produce a smooth output, but requires significant computation time [43]. Figure 5 shows an example of defuzziciation using the center of gravity method. The union of the fuzzy sets is divided into four shaded areas P1, $\mathrm{P} 2, \mathrm{P} 3$, and $\mathrm{P} 4$. The areas (A1, A2, A3, A4) and the center of gravity $\left(x_{1}^{\prime}, x_{2}^{\prime}, x_{3}^{\prime}, x_{4}^{\prime}\right)$ of each area are calculated based on the shape of P1, P2, P3, and P4. Therefore, the center of gravity method can be express using Equation 7 below:

$$
x^{\prime}=\frac{\sum_{i=1}^{n} x_{i}^{\prime} \cdot A_{i}}{\sum_{i=1}^{n} A_{i}}
$$


where $n$ is the number of subareas divided from the union of the fuzzy sets. For the example in Figure 5, the center of gravity is calculated as below:

$$
x^{\prime}=\frac{2.333 \times 1.0+3.917 \times 1.6+5.500 \times 0.6+6.333 \times 0.3}{1.0+1.6+0.6+0.3}=3.943
$$

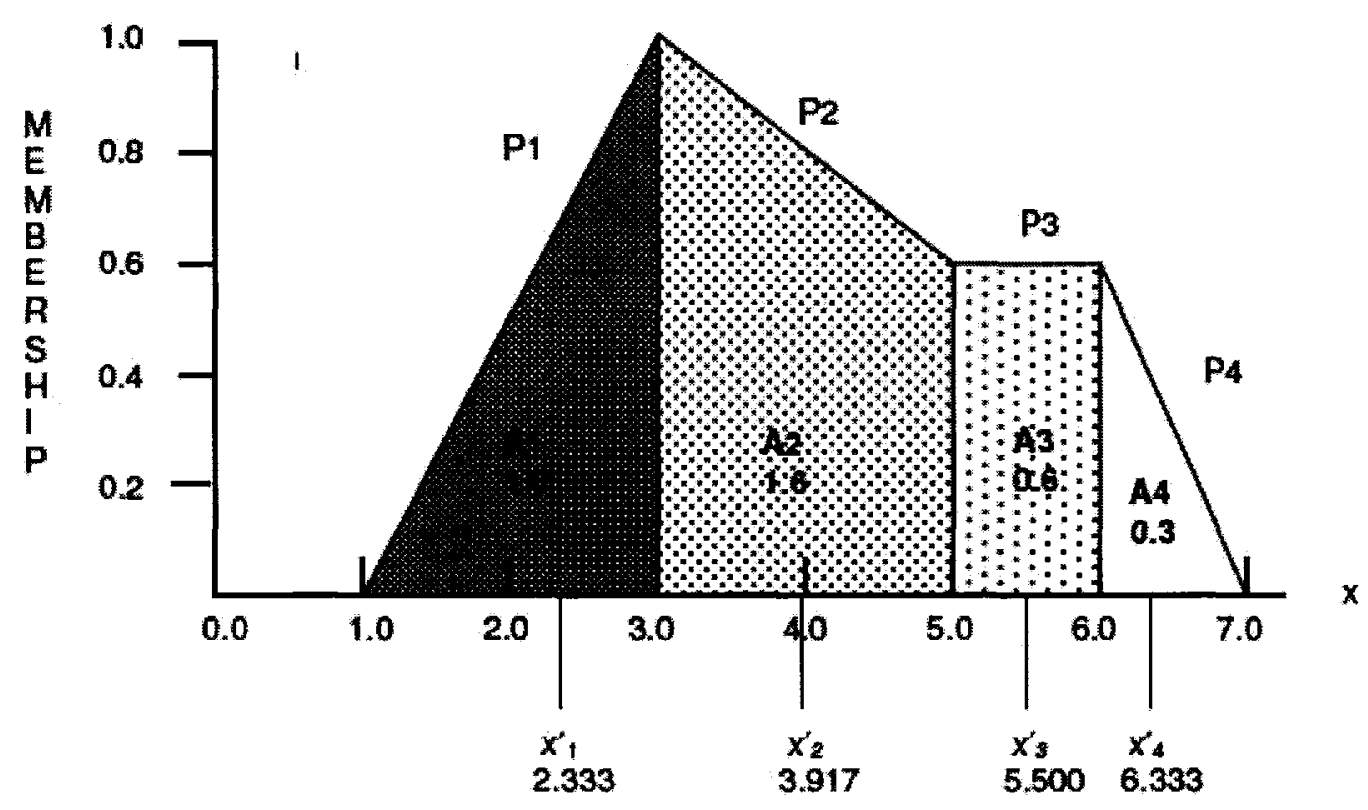

Figure 5: Center of gravity defuzzification method [43] 


\section{C.2 Fuzzy Inference System}

Knowledge-based systems are used to solve problems within a specified domain. A knowledge-based system requires the expert knowledge and thinking patterns in order to be explicitly defined; therefore, this is also known as an expert system. Fuzzy logic is used to construct a knowledge-based system to deal with the problems that occur in satellite communication and that do not have an obvious solution. As a result, this system is also called the Fuzzy Inference System (FIS). FIS has two great advantages as described in [44]. First of all, the FIS is constructed by a set of rules that is translated from expert knowledge. The rules can be written in language that can be understood right away by experts. Second, FIS has the ability to learn by adding new rules. The configuration of FIS is shown in Figure 6. The FIS consists of four functional blocks: fuzzification process, defuzzification process, fuzzy inference engine, and a knowledge base.

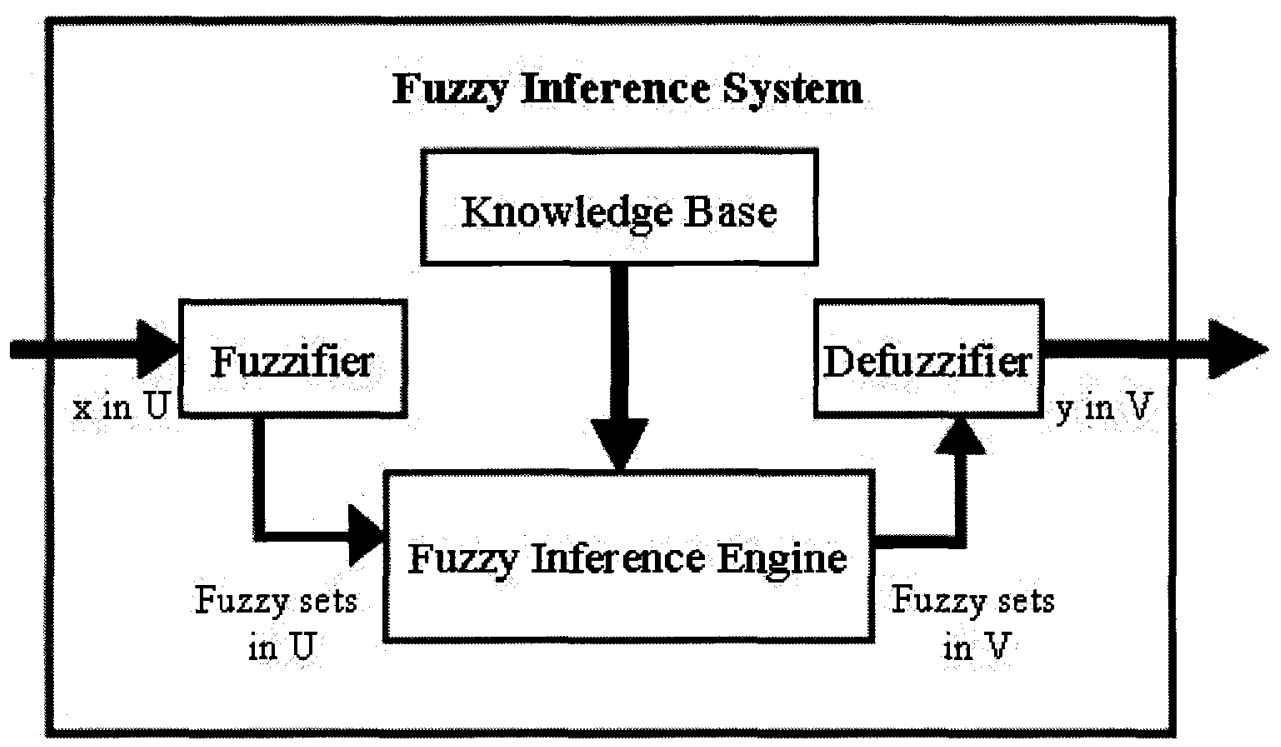

Figure 6: Configuration of Fuzzy Inference System [44] 
Fuzzifier: In the fuzzifier, numerical values of input variables are mapped to fuzzy sets. Hence, the numerical input becomes linguistic variable in the fuzzy inference system. The associated membership function of the fuzzy set provides the degree of truth for the linguistic variable.

Fuzzy Inference Engine: The fuzzy inference engine is the heart of FIS, and it provides decision-making logic to the system. It responds to the linguistic variable provided by the fuzzifier, and it searches the knowledge base in order to find one or more rules upon matching the antecedent. FIS combines the consequent of the rules that have been executed to a union of fuzzy sets. The union of fuzzy sets will be passed onto the defuzzifier in order to be defuzzifized.

Knowledge Base: The knowledge base of the FIS contains domain-specific knowledge that is useful to solve domain related problems. Usually, the knowledge is represented by a set of IF/THEN rules that are translated from human experts.

Defuzzifier: The fuzzy inference engine generates a union of fuzzy sets that includes a set of consequents from executed rules. The union of fuzzy sets is represented by the empirical membership functions. The union of fuzzy sets is defuzzifed using the center of gravity method.

A tipping example is given in order to demonstrate the operability of FIS. In the tipping example, tips to the waitress are based on the quality of food and quality of service. The quality of food and quality of service are the inputs of FIS. The quality of food in a restaurant is represented by a number between 0 to 10 , where 10 is the highest 
score and 0 is the lowest score. The quality of service is also represented by a number between 0 and 10, where 10 means great service and 0 means bad service. The output of FIS is the level of tips to the waitress. The tipping level is represented by a number from 0 to 10 , where 0 means minimum tips should be given and 10 means giving tips generously. The FIS is designed in order to decide the tipping level based on the given quality of food and quality of service. The amount of tips is determined based on the tipping level and the cost of the food. In the following examples, how to decide the tipping level is discussed.

Three fuzzy sets are associated with each input and output variable, where their linguistic expressions are shown in Table 2. The quality of food is express by insipid, ordinary, and delicious; their membership functions are shown in Figure 7. The quality of the service is represented by poor, general, and excellent; their membership functions are illustrated in Figure 8. Figure 9 identifies the membership functions of the fuzzy sets for tips, which are cheap, average, and generous. The rule set of FIS is listed in Table 3 . The rule set in the knowledge base is developed based on common knowledge. For example, rule (3) declares that if the food is delicious and the service is excellent, then the amount of tips should be generous.

Table 2: Associated fuzzy sets for input and output variables

\begin{tabular}{|c|c|}
\hline Variable & Fuzzy Sets \\
\hline Quality of Food & ( Insipid, Ordinary, Delicious) \\
\hline Quality of Service & (Poor, General, Excellent) \\
\hline Tips & (Cheap, Average, Generous) \\
\hline
\end{tabular}


Table 3: Rule set to determine the level of tips

1. If (food is insipid) and (service is excellent) then (Tips is average)

2. If (food is ordinary) and (service is excellent) then (Tips is generous)

3. If (food is delicious) and (service is excellent) then (Tips is generous)

4. If (food is delicious) and (service is general) then (Tips is average)

5. If (food is ordinary) and (service is general) then (Tips is average)

6. If (food is insipid) and (service is general) then (Tips is cheap)

7. If (food is delicious) and (service is poor) then (Tips is average)

8. If (food is ordinary) and (service is poor) then (Tips is cheap)

9. If (food is insipid) and (service is poor) then (Tips is cheap)

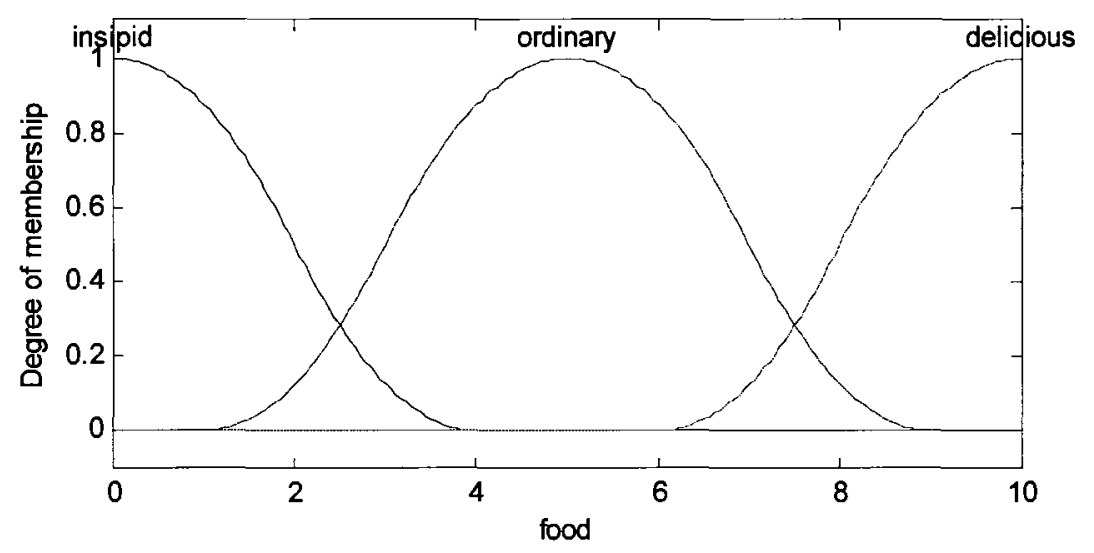

Figure 7: Membership functions for quality of food

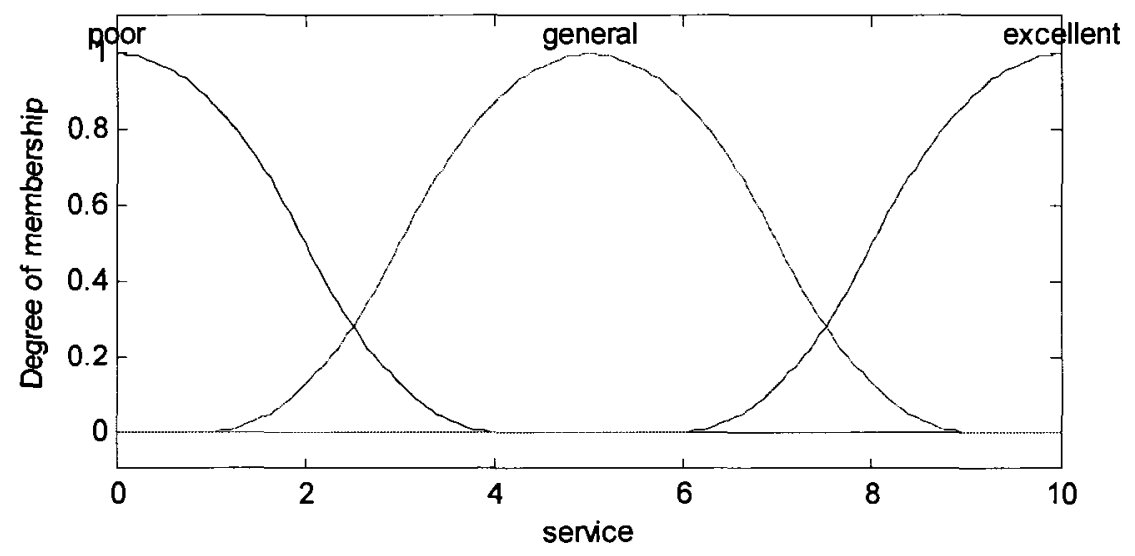

Figure 8: Membership functions for quality of service 


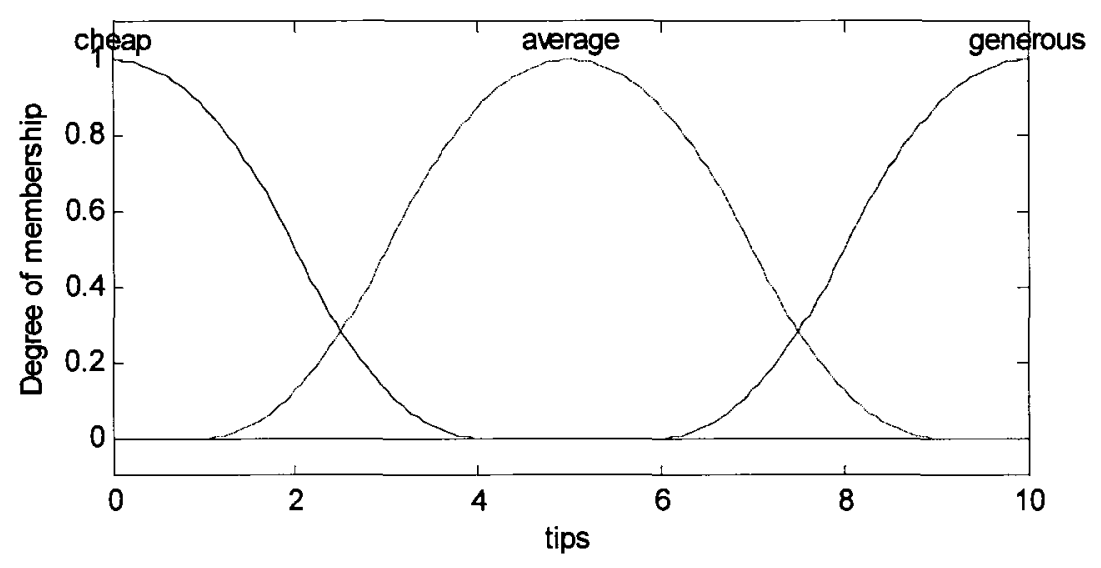

Figure 9: Membership functions for tips

At the beginning of each decision making process, the numerical inputs of FIS will be mapped to linguistic variables according to the membership functions shown in Figure 8 and Figure 9. The rule set in the knowledge base will be executed upon matching the input linguistic variables. A union of fuzzy sets is generated depending on the rules being executed. In the last step, the union of the fuzzy sets is defuzzified using the centre of gravity method. Two examples are given below in order to help explain how FIS works with different numerical inputs.

In the first example, suppose both the quality of the food and the quality of the service in the restaurant ranks 5 out of 10 . According to the pre-defined membership functions shown in Figure 8 and Figure 9, the quality of the food can be represented by the linguistic variable "ordinary" with a degree of truth equal to 1 and a quality of service can be represented by linguistic variable "general" with a degree of truth equal to 1 . The input linguistic variables match the antecedent of rule (5) from Table 3; therefore, rule (5) is executed. The consequent of rule (5) is shown in Figure 10. Because only one rule is executed, the union of fuzzy sets only consist of consequent on rule (5). After defuzzifing 
the union of fuzzy sets, the level of tips to the waitress is 5 , which means "average" amount of tips should be given.

5

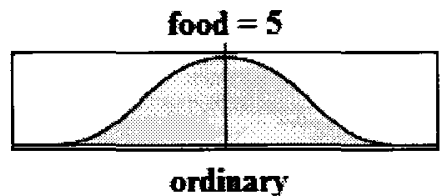

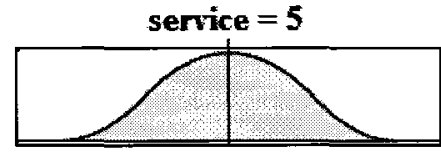

general

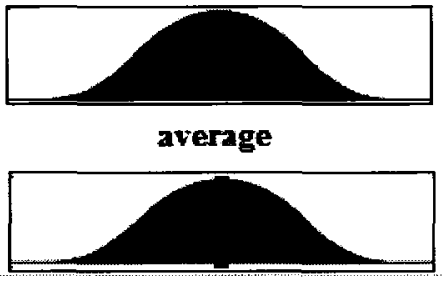

tips $=5$

Figure 10: Consequence of rule (5) being executed

In most cases, the input variables belong to more than one fuzzy set. Thus, they can be represented by more than one linguistic variable with a different degree of truth. The following example shows how FIS works when an input variable is represented by two linguistic variables. When visiting a restaurant, suppose that the quality of the food is 5 , and the quality of the service is 8 . The quality of the food can be represented by the linguistic variable "ordinary" with degree of truth equals to 1 . According to the definition of the membership function for quality of service, the value 8 belongs to both the "general" and "delicious" categories with different degrees of truth. As a result, rule (2) and (5) from Table 3 will be executed. The consequents of rule (2) and rule (5) are shown in Figure 11. The union of the fuzzy sets of rule (2) and (5) being executed is defuzzified, and the level of tips should be given to the waitress is 7.24 out of 10 , which means the amount of tip given should be "above average".

Figure 12 uses a 3 dimension graph to display the decision surface of the tipping FIS. The $\mathrm{X}$-axis and the $\mathrm{Y}$-axis are the quality of food and the quality of service. The Z-axis displays the tipping level. 


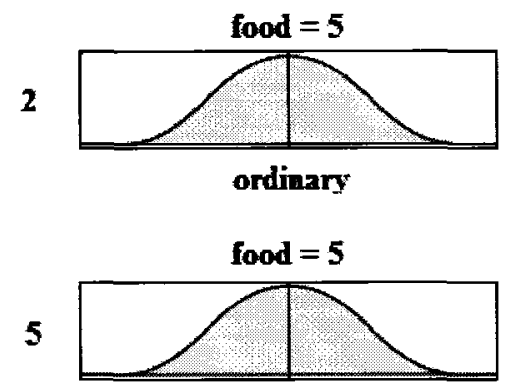

ordinary
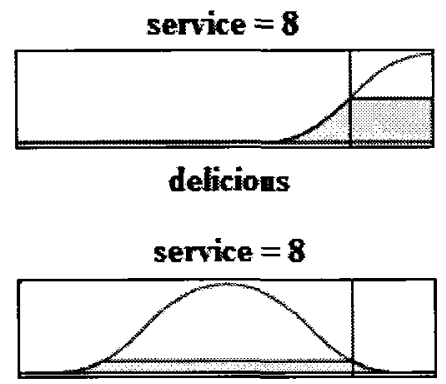

general

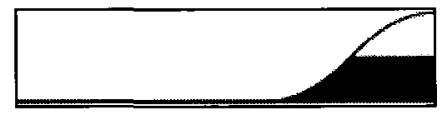

excellent

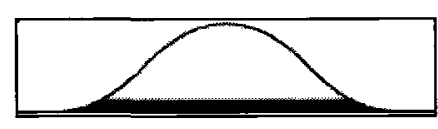

average

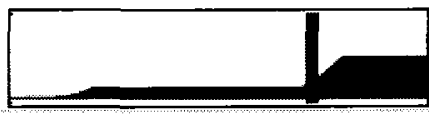

tips $=7.24$

Figure 11: Consequence of rule (2) and (5) being executed

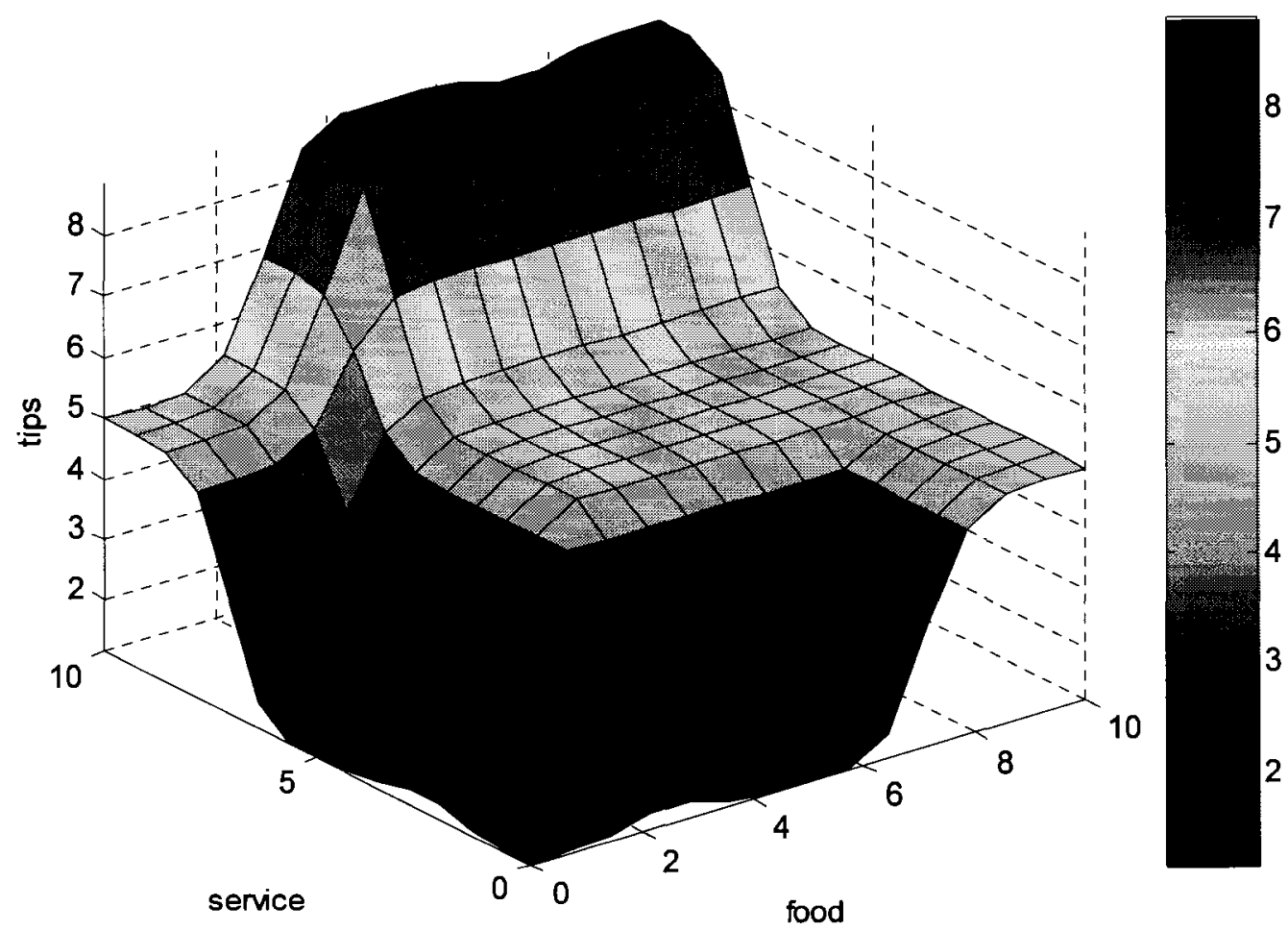

Figure 12: Decision surface of tipping FIS 


\section{Appendix D: Historical Forward Link SNR}

List of Available Historical Forward Link SNR:

\begin{tabular}{|c|c|}
\hline Station & Duration \\
\hline Altin & $\begin{array}{c}\text { November 2006 to July 2007 } \\
\text { (Without February 2007) }\end{array}$ \\
\hline Hazelton & February 2007 to December 2007 \\
\hline Terrace & August 2007 to December 2007 \\
\hline
\end{tabular}



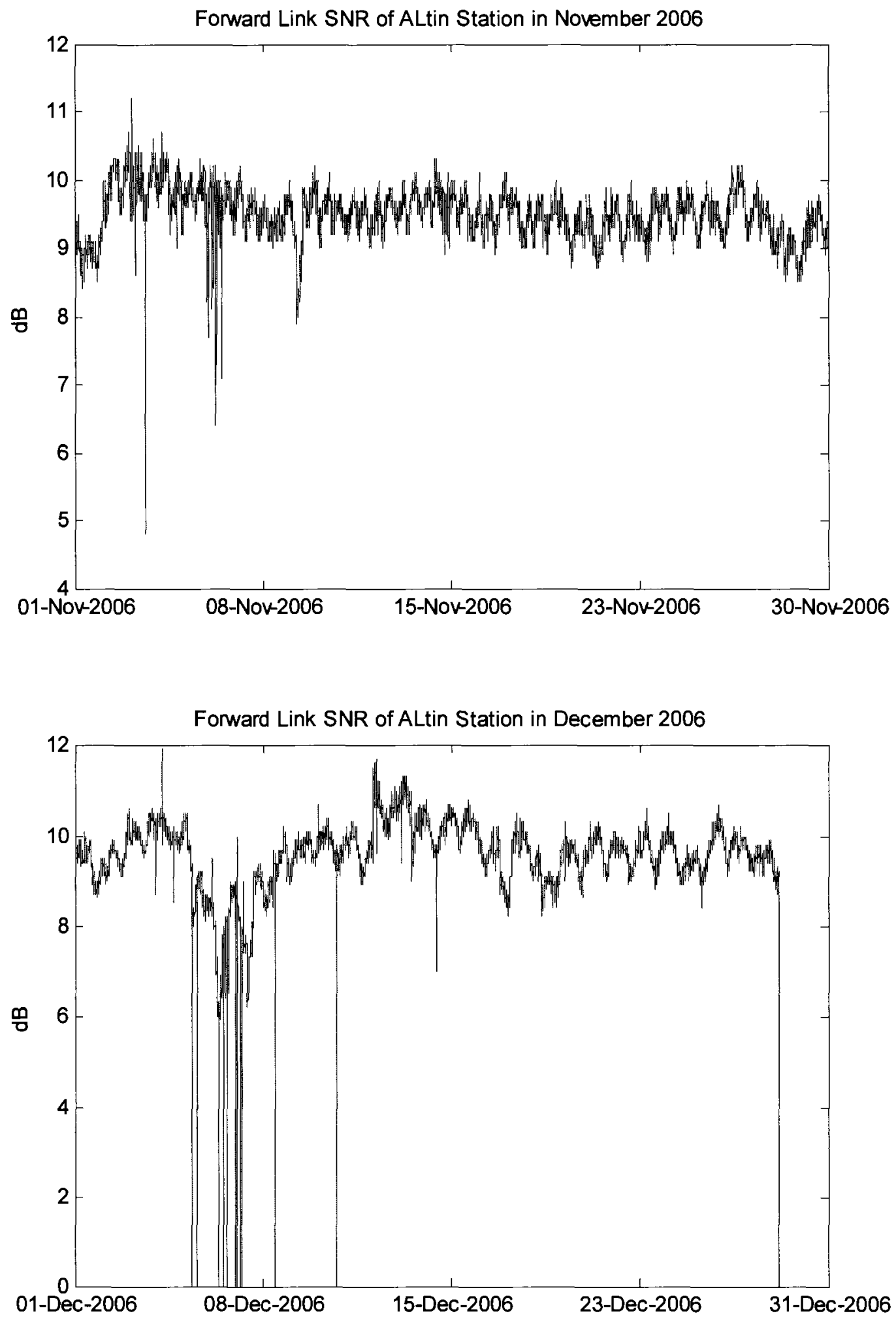

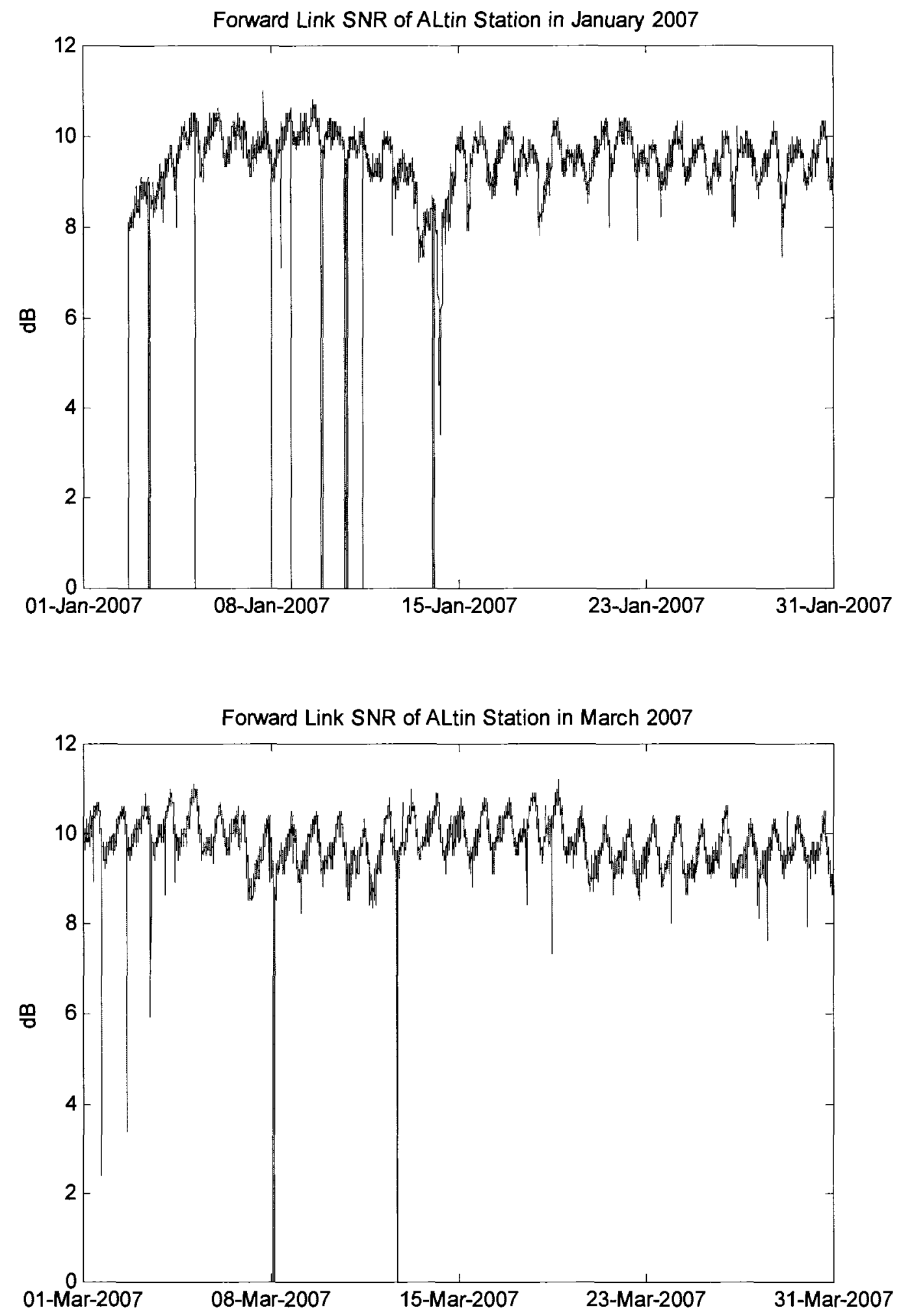

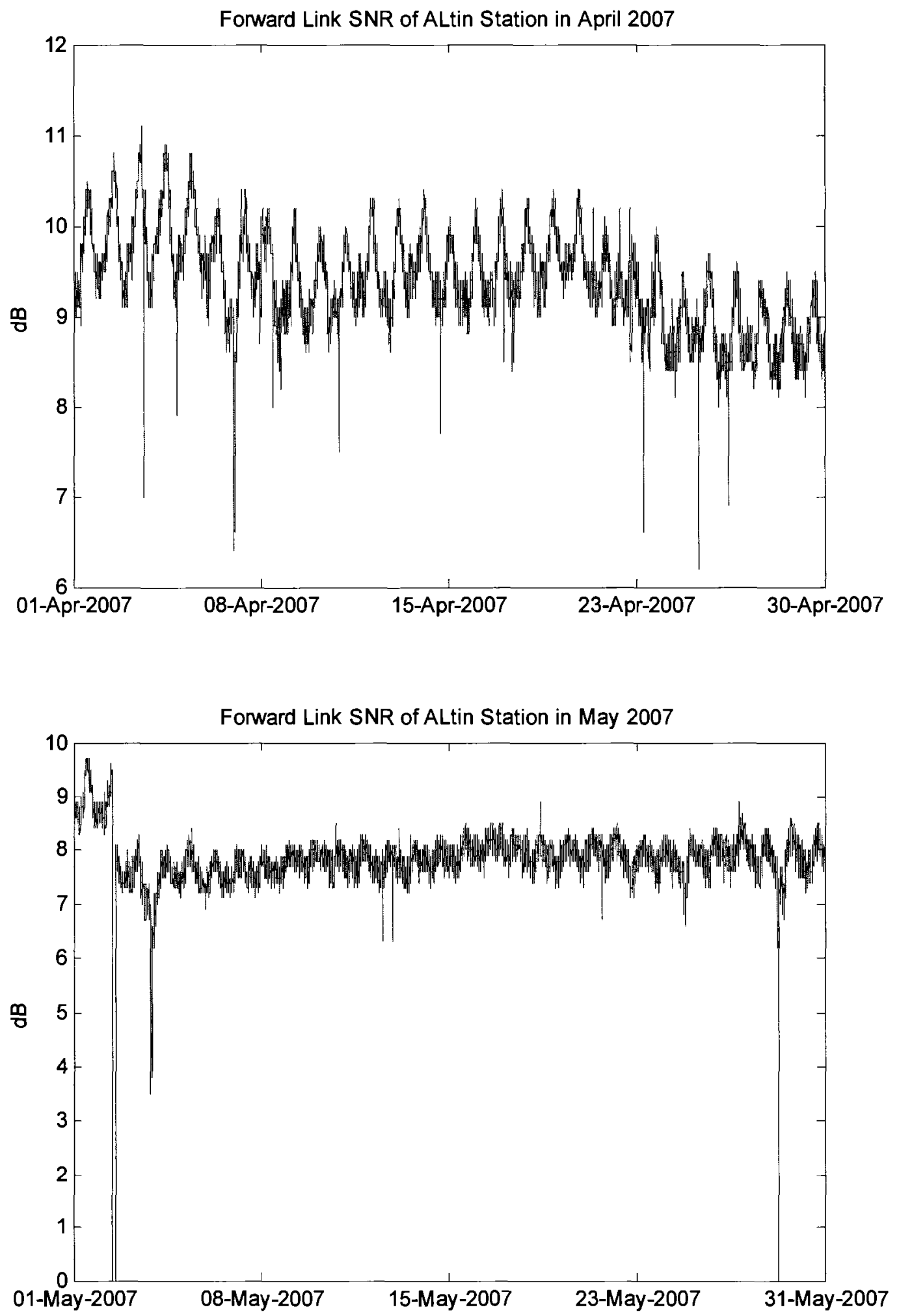

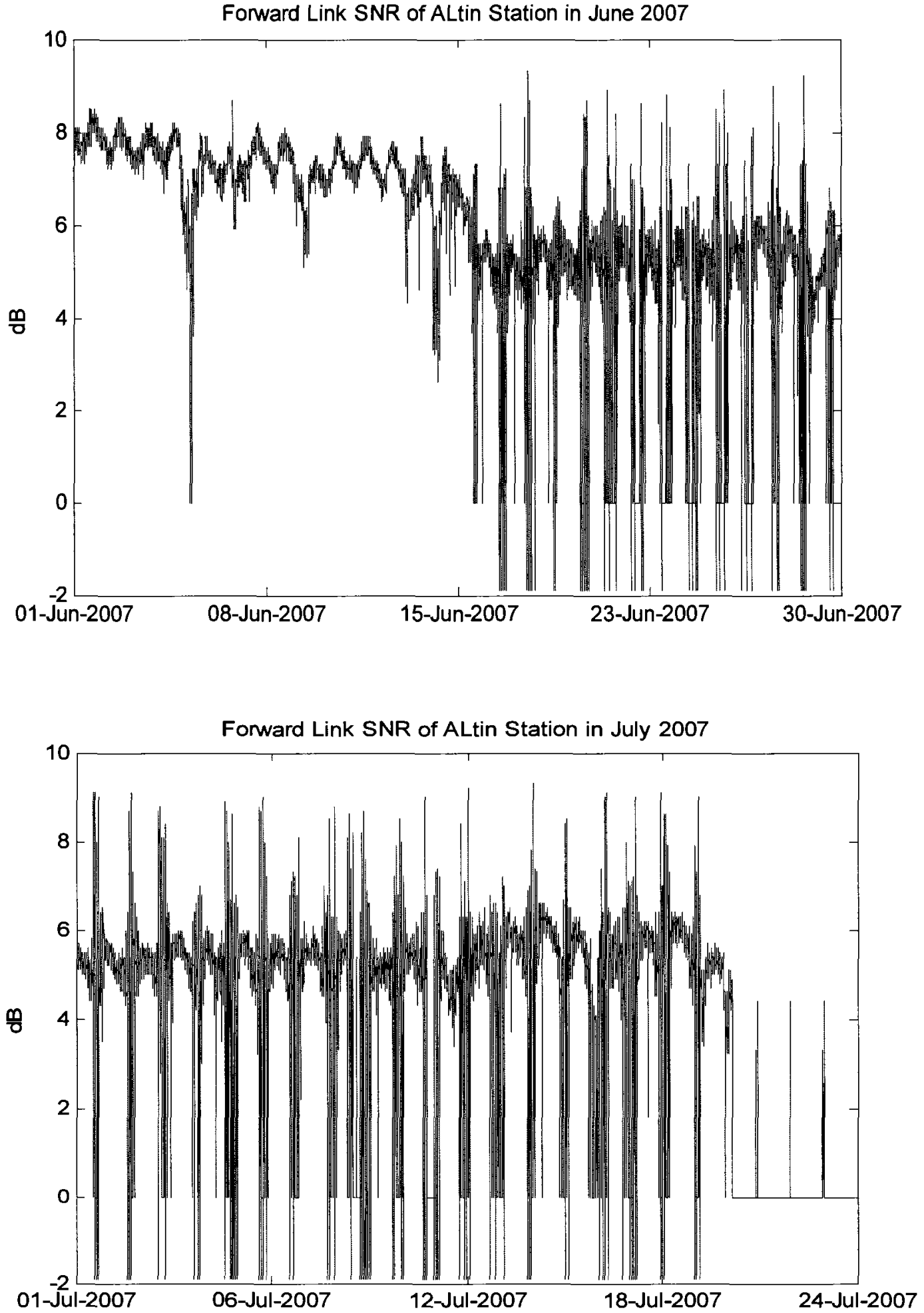

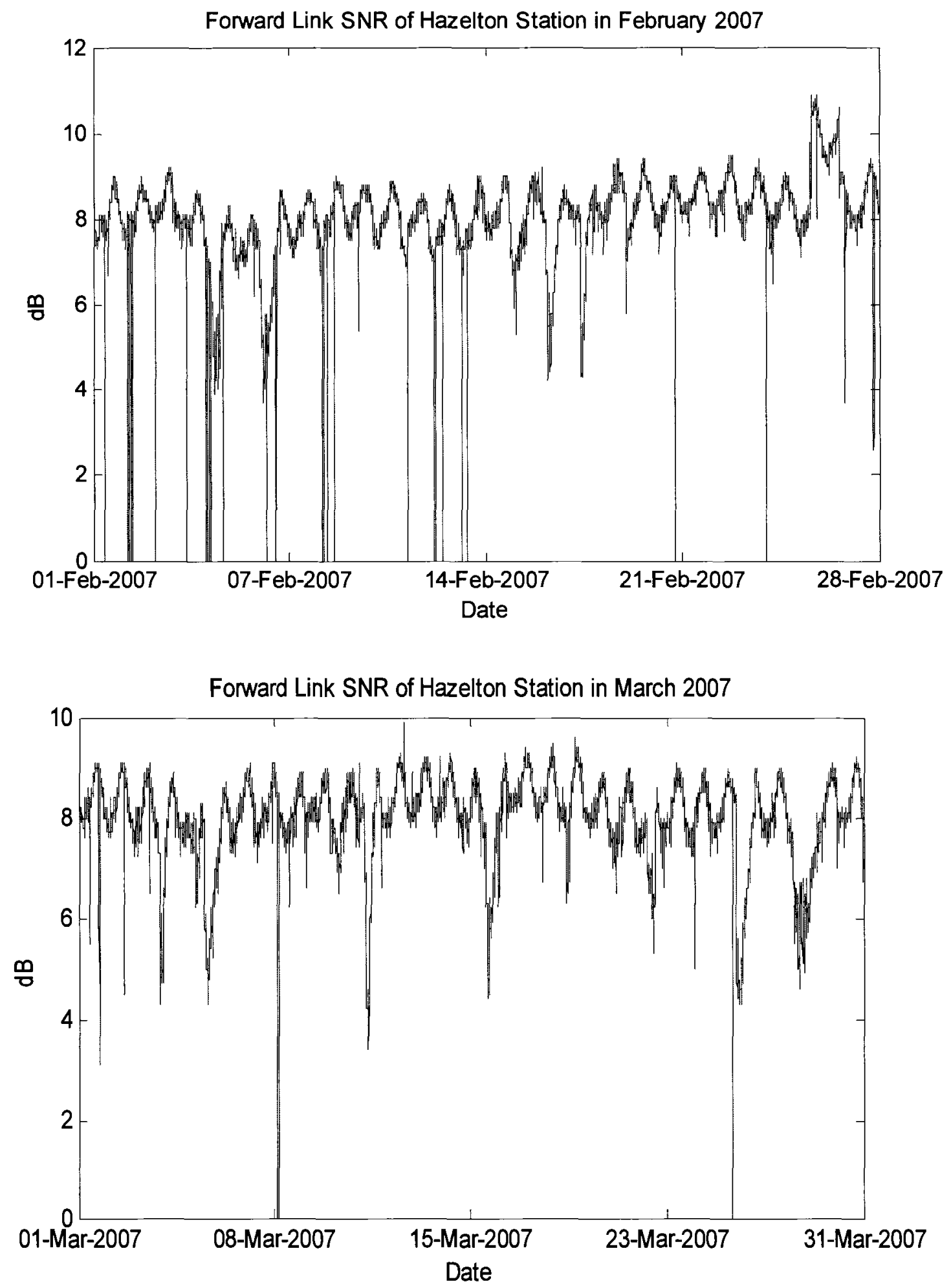
Forward Link SNR of Hazelton Station in April 2007
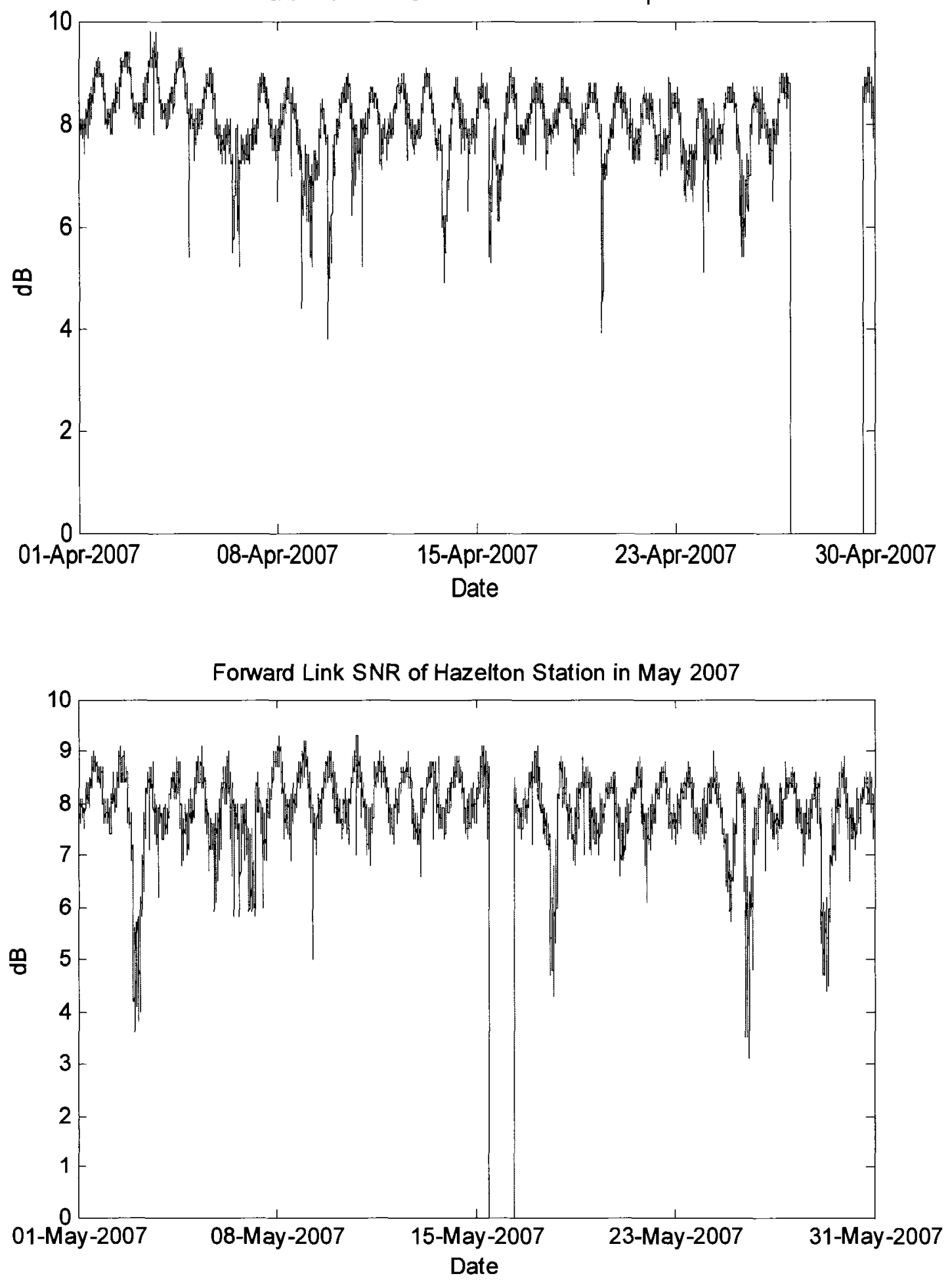

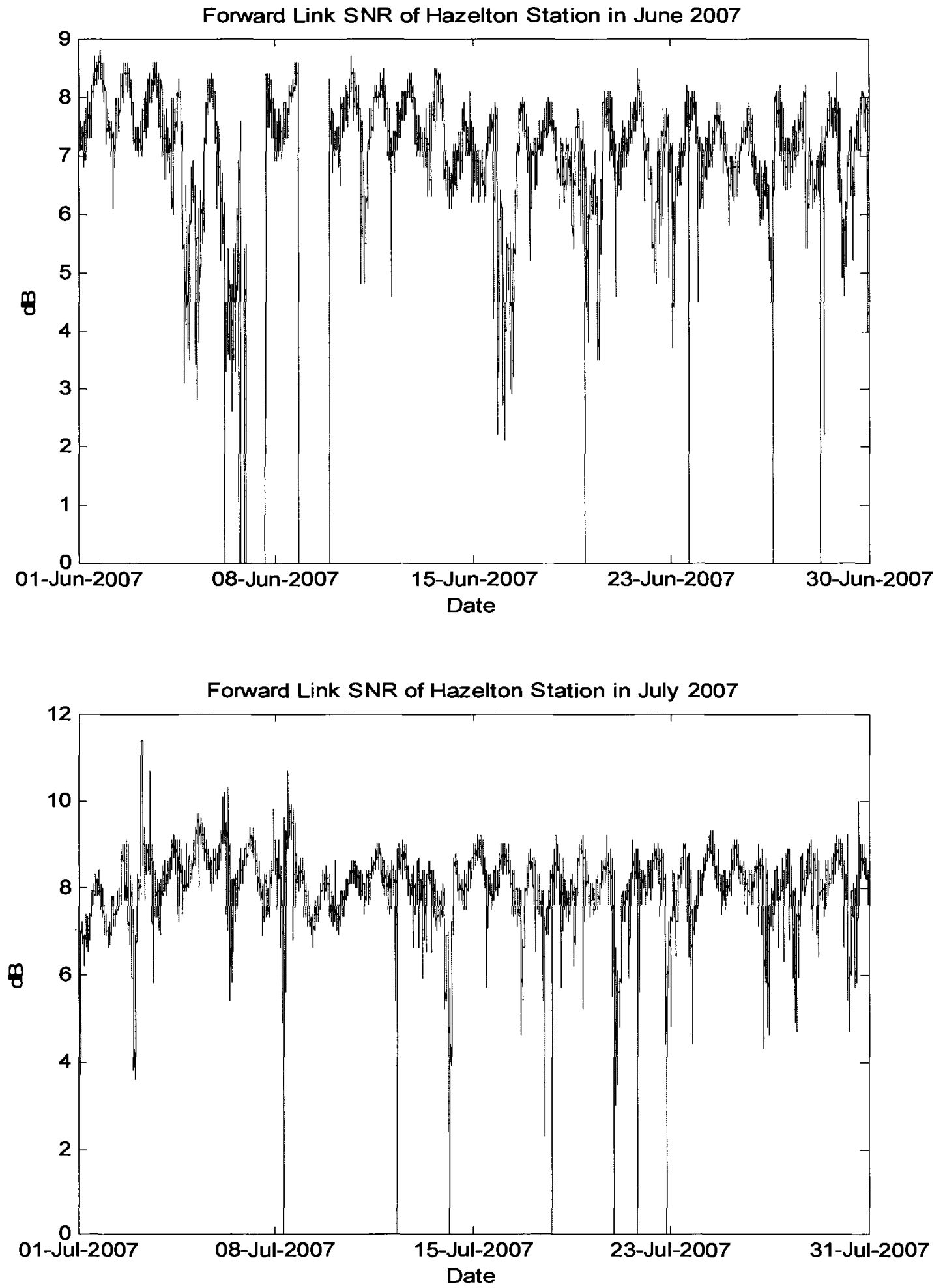

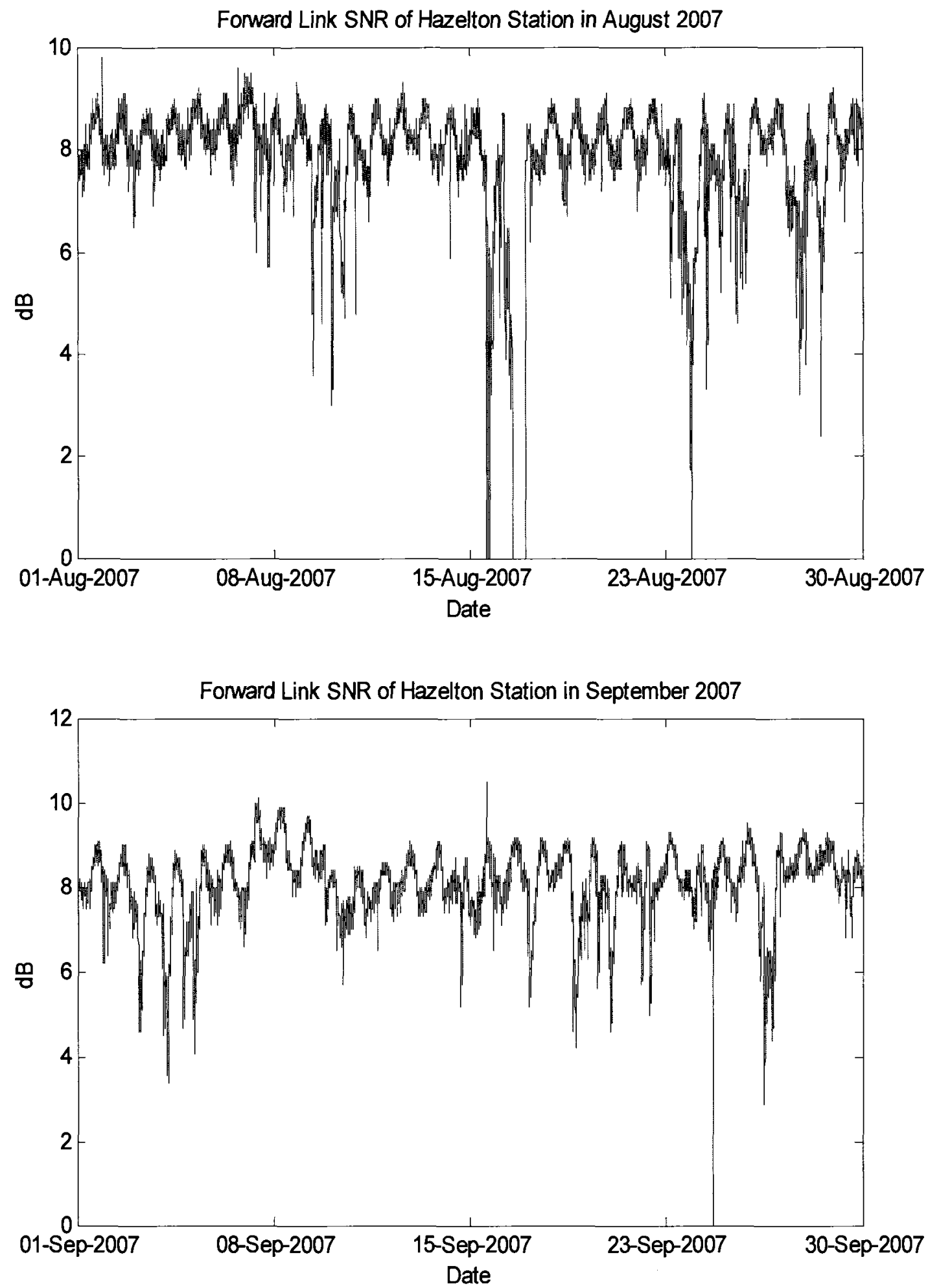

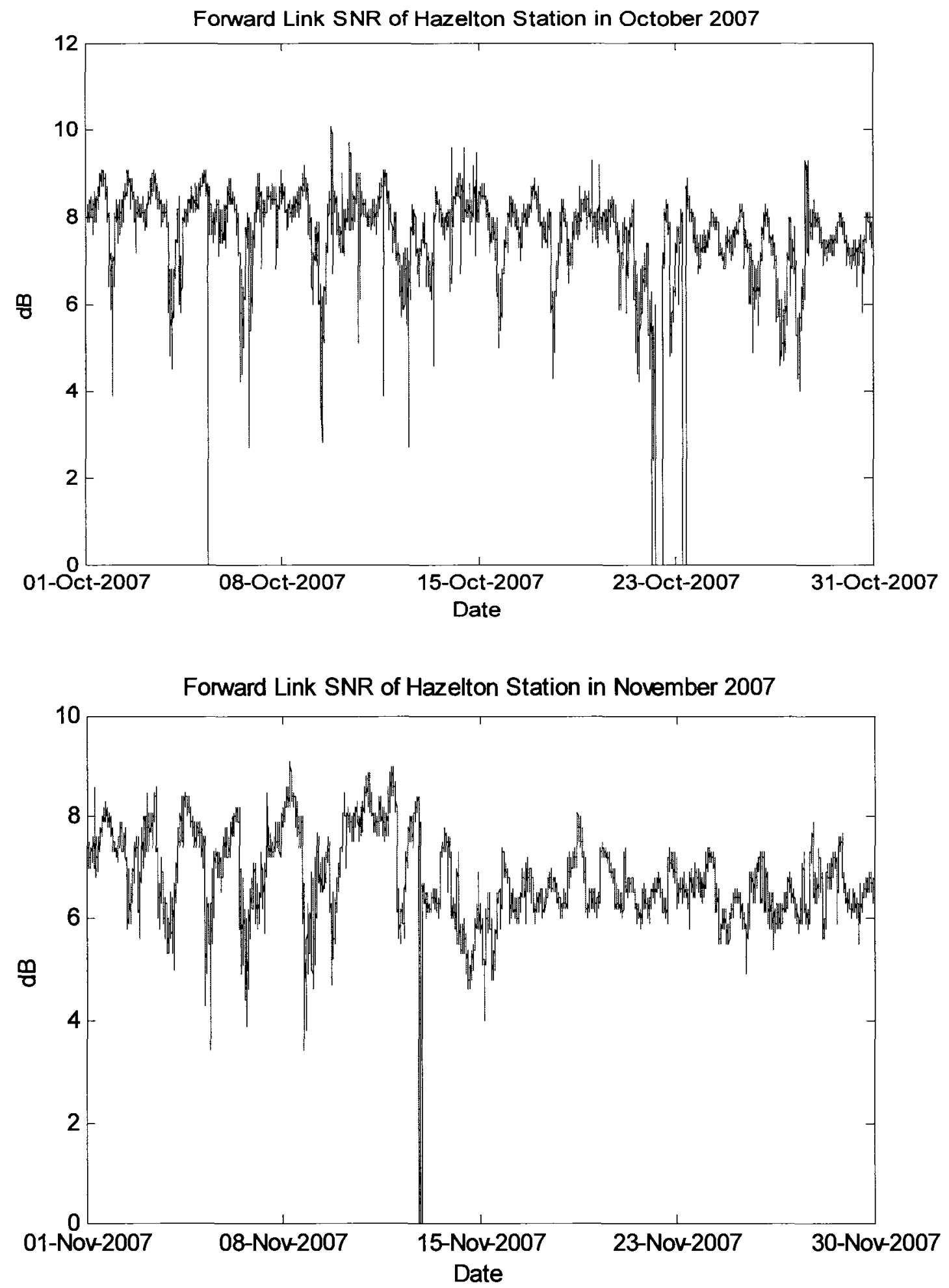


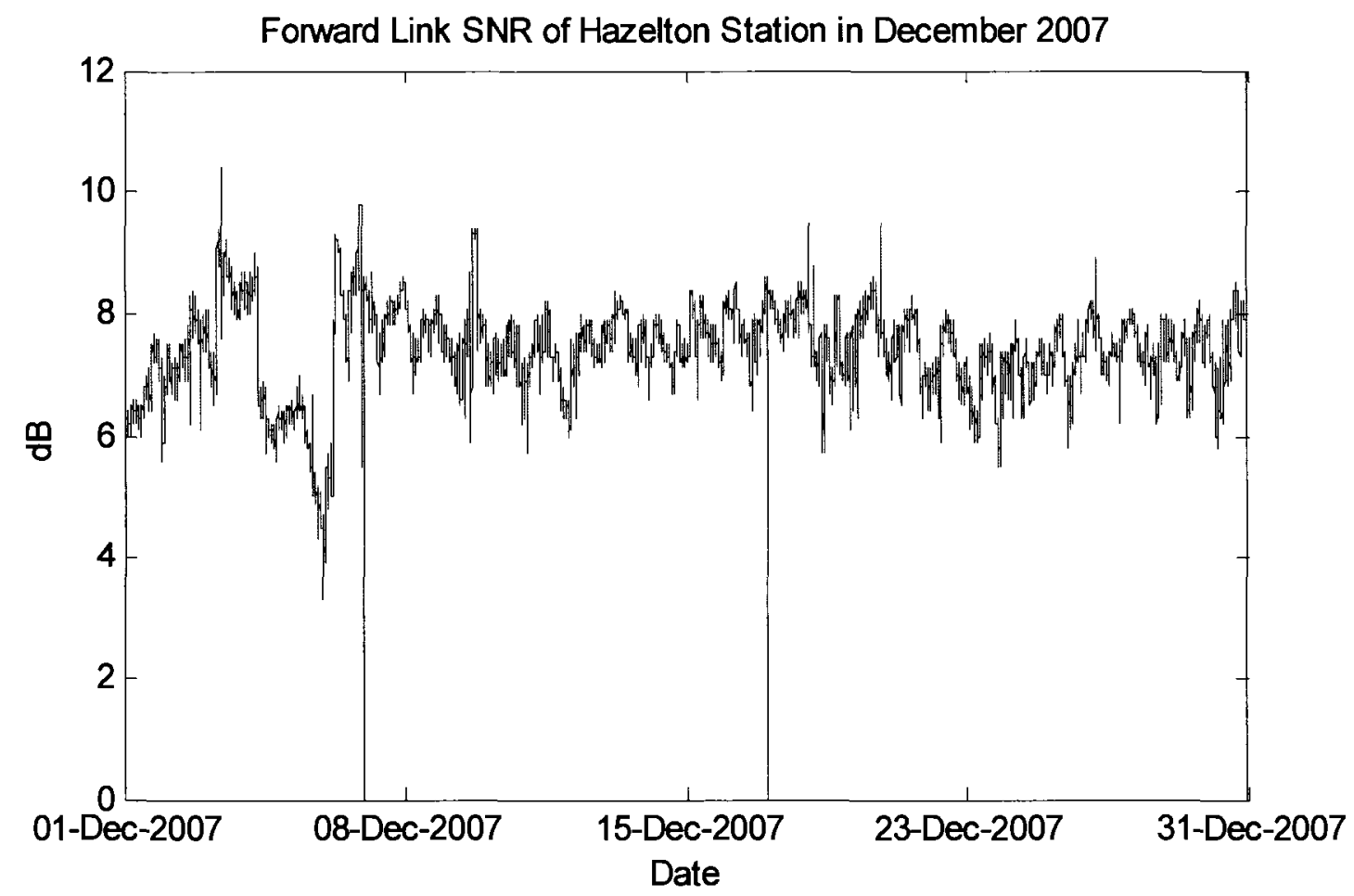



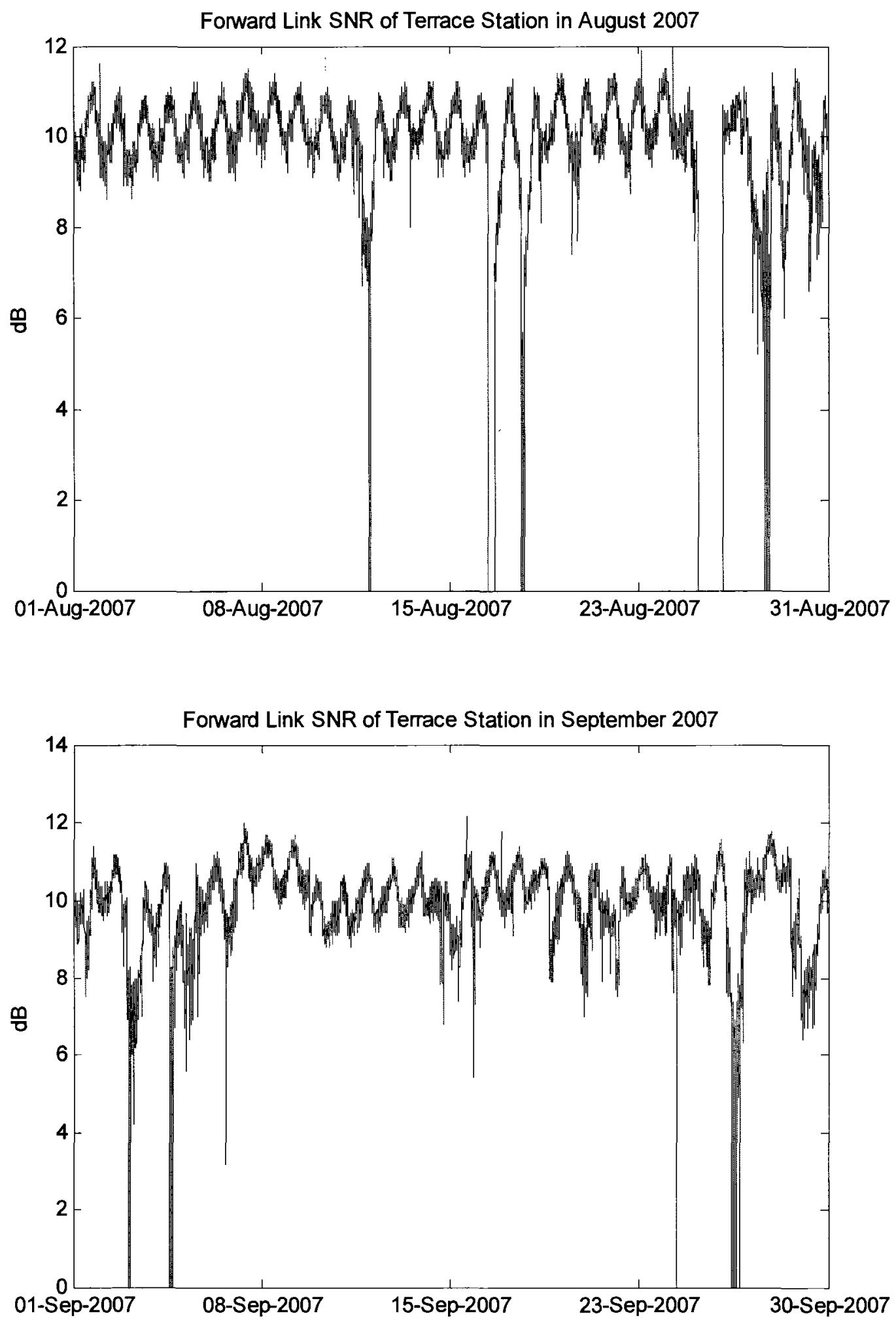

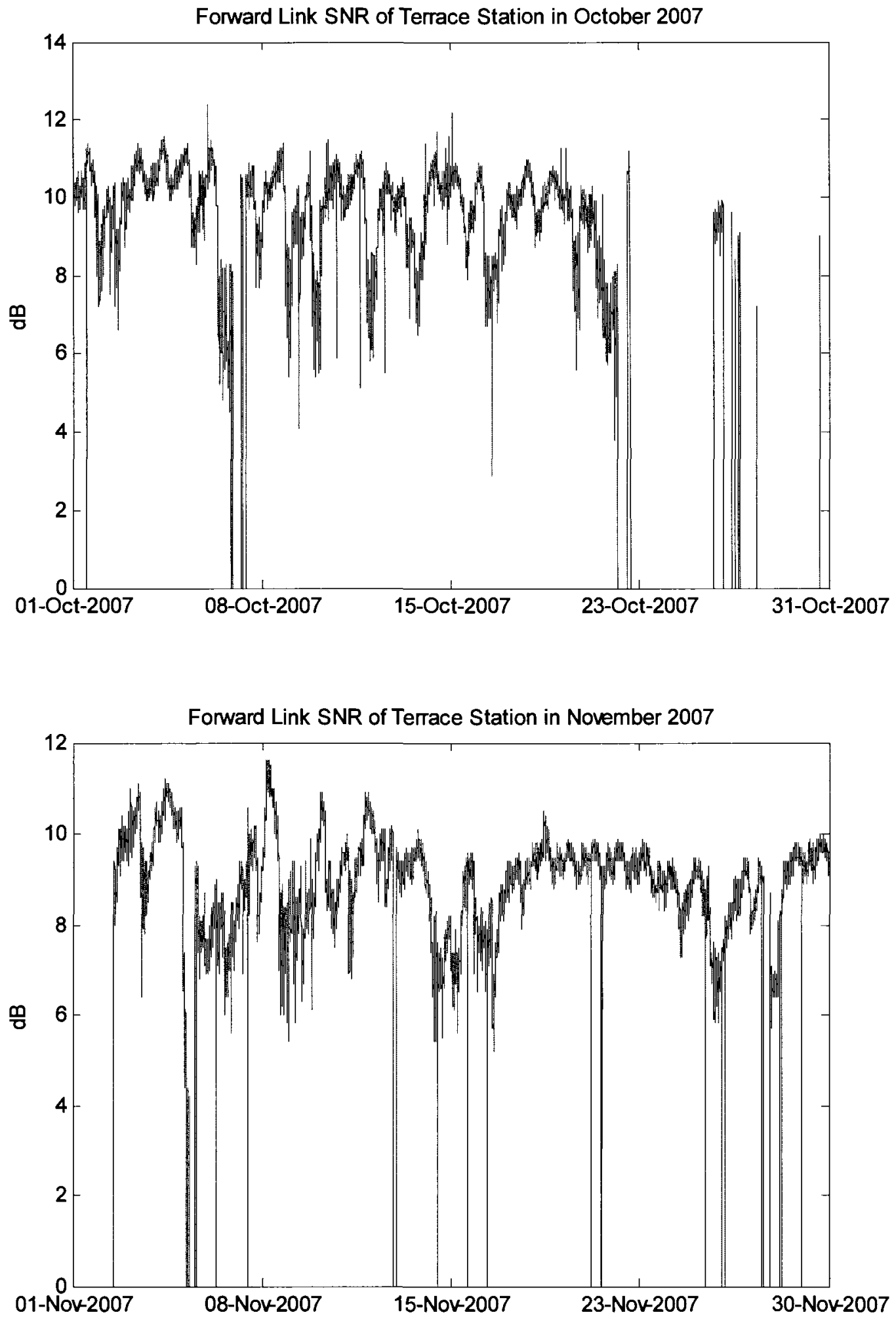


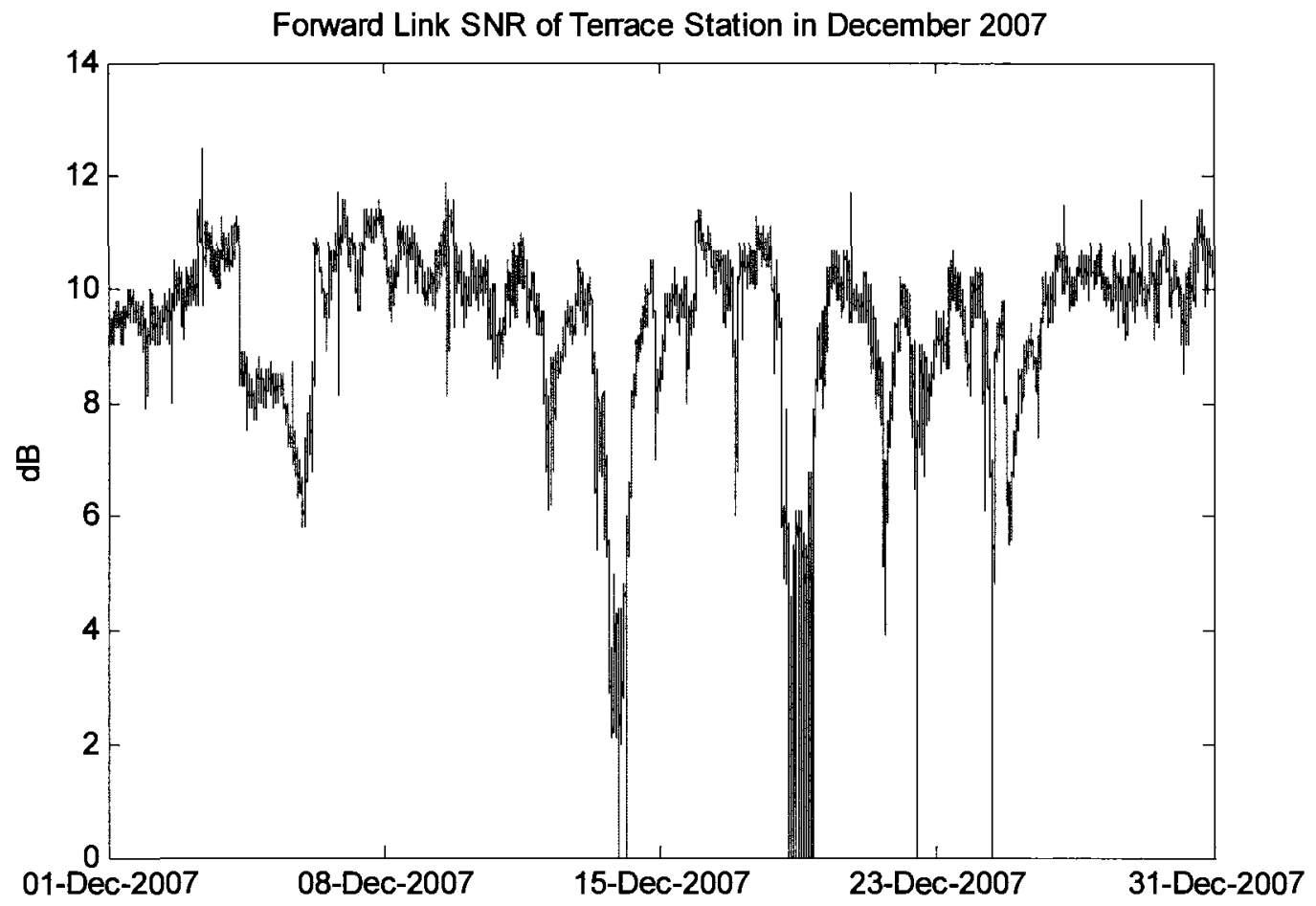




\section{References}

[1] A. Jamalipour, "Broad-band satellite networks - The global IT bridge", Proceedings of the IEEE, Vol. 89, No.1, pp.88-104, January 2001

[2] A. Grami and K. Gordon, "Next-generation Ka-band satellite concept to extend the reach of Canada's broadband infrastructure", Proceedings of the IEEE Global Telecommunications Conference 2001, Vol. 4, pp.2754-2758, San Antonio, November 2001

[3] A. Grami, K. Gordon and A. Shoamanesh, "Anik F2 Ka-band System: High-Speed Internet Access", Proceedings of 18th ALAA International Communication Satellite Systems Conference 2000, pp. 1231-1238, Oakland, April 2000

[4] "Anik F2: Ka-band for North America", http://www.boeing.com/defensespace/space/bss/factsheets/702/anik_f2/anik_f2.html Broadband Infrastructure

[5] "Interactive Coverage Map", http://www.telesat.ca/satellites/index-e.asp

[6] K. Harb, A. Srinivasan, C. Huang and B. Cheng, "QoS In Weather Impacted Satellite Networks", Proceedings of IEEE Pacific Rim Conference on Communication, Computers and Signal 2007, pp.178-181,Victoria, August 2007

[7] T. Pratt, C. Bostian and J. Allnutt, "Satellite Communications", Second Edition., John Wiley \& Sons, 2003

[8] M. R. Chartrand, "Satellite Communications for the Nonspecialist", SPIE Press, 2004

[9] F. Davarian, "Ka-band Propagation Research Using ACTS", International Journal of Satellite Communication, Vol.14, No. 3, pp 267-282, October1995

[10]R. Bauer, "Ka-band propagation measurements: an opportunity with the Advanced Communications Technology Satellite (ACTS)", Proceedings of the IEEE, Vol. 85, No.6, pp.853-862, June 1997 
[11]L. Mamatas, T. Harks and V. Tsaoussidis, "Approaches to Congestion Control in Packet Networks", Journal of Internet Engineering, Vol. 1, Issue 1, Kleidarithmos Press, 2007

[12]D. Chiu and R. Jain, "Analysis of the Increase/Decrease Algorithms for Congestion Avoidance in Computer Networks", Journal of Computer Networks and ISDN, Vol. 17, No. 1, pp. 1-14, June 1989

[13] W. Prue and J. Postel, "Something a host could do with source quench", IETF RFC 1016, Networking Working Group, July 1987

[14] V. Jacobson, "Congestion Avoidance and Control", ACM SIGCOMM Computer Communication Review, Vol. 25, pp. 157-187, January 1995

[15]U. Mukherii, "A schedule-based approach for flow-control in data communication networks", Ph.D. Thesis, Massachusetts Institute of Technology, February 1986

[16]K. Ramakrishnan and R. Jain, "A binary feedback scheme for congestion avoidance in computer network with a connectionless network layer", ACM SIGCOMM Computer Communication Review, Vol. 18, No. 4, pp.303-313, August 1988

[17]D. Comer and R. Tavatkar, "A rate-based congestion avoidance and control scheme for packet switched networks", Proceedings of $10^{\text {th }}$ IEEE International Conference on Distributed Computing Systems 1990, pp. 390-397, Paris, May1990

[18]B. Braden, D. Clark, J. Crowcroft, B. Davie, S. Deering, D. Estrin, S. Floyd, V. Jacobson, G. Minshall, C. Partridge, L. Peterson, L. Ramakrishnan, S. Shenker, J. Wroclawski and L. Zhang, "Recommendations on Queue Management and Congestion Avoidance in the Internet", IETF RFC 2309, April 1998

[19]S. Floyd and V. Jacobson, "Random Early Detection Gateways for Congestion Avoidance", IEEE/ACM Transactions on Networking, Vol. 1, No. 4, pp. 397-413, 1993

[20] Y. Fan, F. Ren and C. Lin, "Design a PID controller for active queue management", Proceedings of $8^{\text {th }}$ IEEE International Symposium on Computers and Communication 2003, pp. 985-990, Kemer-Antalya, June 2003

[21] A. Bitorika, M. Robin and M. Huggard, "An evaluation framework for active queue management schemes", Proceedings of $11^{\text {th }}$ IEEE/ACM International Symposium on Modeling, Analysis and Simulation of Computer Telecommunications Systems 2003, pp.200-206, Orlando, October 2003 
[22]D. Katabi, M. Handley and C. Rohrs, "Congestion control for high bandwidth-delay product networks", Proceedings of ACM Conference on Special Interest Group on Data Communication 2002, pp.19-23, Pittsburgh, August 2002

[23] V. Paxon and S. Floyd, "Wide area traffic: the failure of Poisson modeling", IEEE/ACM Transaction on Networking, Vol. 3, No. 3, pp.226-244, March 1995

[24]S. Ghosh, Q. Razouqi, H. J. Schumacher and A. Celmins, "A survey of recent advances in fuzzy logic in telecommunications networks and new challenges", IEEE Transactions on Fuzzy Systems, Vol.6, No.3, pp.443-447, August 1998

[25] T. Hadjadj Aoul, A. Nafaa, D. Negru and A. Mehaoua, "FAFC: fast adaptive fuzzy AQM controller for TCP/IP networks", Proceedings of IEEE Globe Telecommunication Conference 2004, Vol.3, pp.1319-1323, Dallas, December 2004

[26] H. H. Lim and B. Qiu, "Fuzzy logic traffic control in broadband communication networks", Proceedings of IEEE International Fuzzy Systems Conference 2001, Vol. 1, pp. 99-102, Melbourne, December 2001

[27]S. Tanenbaum, "Computer Networks", Fourth Edition, Prentice Hall, March 2003

[28] M. May, T. Bonald and T. Bolot, "Analytic evaluation of RED performance", Proceedings of $19^{\text {th }}$ Annual Joint Conference on the IEEE Computer and Communications Societies 2000, Vol. 3, pp. 1415-1424, Tel Aviv, March 2000

[29] H. Ohsaki, M. Murata and H. Miyahara, "Steady state analysis of the RED gateway: stability, transient behavior, and parameter setting", IEICE Transactions on Communications, Vol. E85-B, January 2002

[30]F. Ren, Y. Ren and X. Shan, "Design of a fuzzy controller for active queue management", Computer Communications, Vol.25, No. 9, pp. 874-883, June 2002

[31] Y. Fan, F. Ren and C. Lin, "Design an Active Queue Management Algorithm Based on Fuzzy Logic Decision", Proceedings of International Conference on Communication Technology 2003, Vol. 1, pp. 286-289, Beijing, April 2003

[32]C. Chrysostomou, A. Pitsillides, L. Rossides and A. Sekercioglu, "Fuzzy logic controlled RED : congestion control in TCP/IP differentiated services networks", Journal of Soft Computing - A Fusion of Foundations, Methodologies and Applications, Vol. 8, No. 2, pp. 79-92, 2003

[33] B. Vallamsundar, J. Zhu, K. Ponnambalam, C. Huang and B. Cheng, "Adaptation to Congestion with Intelligent System under Dynamic Weather Conditions", Proceedings of International Symposium on Signals, Systems and Electronics 2007, pp. 415-418, Montreal, August 2007 
[34]D. C. Elmore and T. R. Hurd, "Fault detection and fault isolation in the ground station", Proceedings of Military Communications Conference 1997, Vol. 3, pp.1448-1452, Monterey, November 1997

[35]P. Lazaro, R. Barco and J. Hermoso, "Diagnosis of earth stations using Bayesian networks", Proceedings of International Conference on Artificial Intelligence and Applications 2002, pp. 268-272, Malaga, September 2002

[36]X. Hou, Z. Fan, Lei. Li, F. Xu, C. She and G. Hu, "Algorithm of fault diagnosis for satellite network", Proceedings of the 1997 IEEE International Conference on Intelligent Mechatronics and Automation 2004, pp. 594-598, Chengdu, August 2004

[37]L. Elerin, C. Learoyd and B. Wilson, "Applying neural networks and other AI techniques to fault detection in satellite communication systems", Proceedings of the IEEE Workshop on Neural Networks for Signal Processing VII, pp.617-625, Amelia Island, September 1997

[38] M. B. Green and P. R. Sims, "An expert aid to fault diagnosis and trouble-shooting in satellite communications", Journal of Electronics \& Communication Engineering, Vol.3, No.6, pp.252-258, December 1991

[39]R. Schlegelmich, J. Durkin and E. Petrik, "GTEX: An Expert System for Diagnosing Faults in Satellite Ground Stations", NASA Lewis Research Center, November 1991

[40]L. Xu and C. Huang, "Wavelet-Based SNR Analysis in Building Satellite Terminal Fault Identification System", Proceedings of IEEE International Conference on Communication 2008, pp.1942-1946, Beijing, May 2008

[41] L. A. Zadeh, "Fuzzy sets", Information and Control, Vol.8, pp.338-353, 1965

[42] W. Siler and J. J. Buckley, "Fuzzy Expert Systems and Fuzzy Reasoning", John Wiley \& Sons Inc., 2005.

[43]R. A. Orchard, "Fuzzy CLIPS User's Guide", Integrated Reasoning Institute for Information Technology, National Research Council Canada, October 1998

[44]L. Wang, "A Course in Fuzzy System and Control”, Prentice Hall, 1997.

[45] Information Science Institute, University of Southern California, "Internet Protocol", IETF RFC 791, September 1981

[46]K. K. Ramakrishnan and S. Floyd, "A Proposal to add Explicit Congestion Notification (ECN) to IP”, IETF RFC 2481, January 1999 
[47] K. K. Ramakrishnan, S. Floyd and D. L. Black, "The addition of explicit congestion notification (ECN) to IP", IETF RFC 3168, September 2001

[48] J. Banks, J. S. Carson, B. L. Nelson and D. M. Nicol, "Discrete-Event System Simulation", Fourth Edition, Prentice Hall, 2004

[49]B. Sklar, "Digital Communications: Fundamentals and Applications", Second Edition, Prentice Hall, 2001

[50]T. S. Rappaport, "Wireless Communications: Principles and Practice", Second Edition, Prentice Hall, 2002 